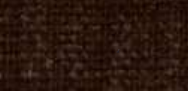

(4)

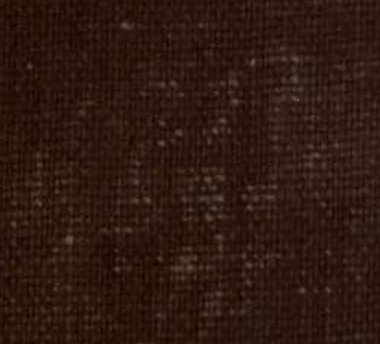

-

2005

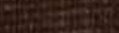

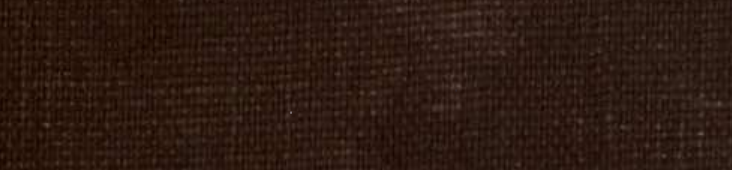

5is

3.

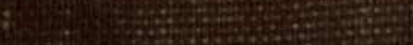

4 4.

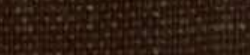

W

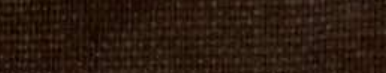




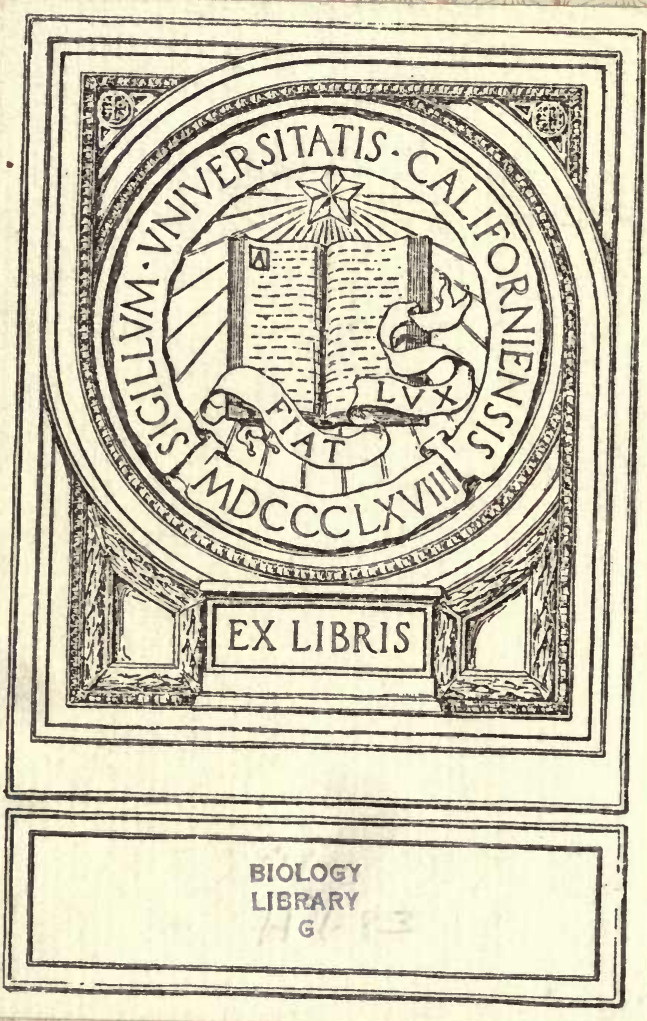





\section{- DEVELOPMENT}

\section{AND PURPOSE.}

AN ESSAY TOWARDS

A PHILOSOPHY OF EVOLUTION

\section{BY \\ L. T. HOBHOUSE}

MARTIN WHITE PROFESSOR OF SOCIOLOGY IN THE UNIVERSITY OF LONDON FORMERLY FELLOW AND ASSISTANT-TUTOR OF CORPUS CHRISTI COLLEGE, OXFORD

MACMILLAN AND CO., LIMITED ST. MARTIN'S STREET, LONDON 
QH366

H 6

BIOLOGY

LIBRARY

COPYRIGHT

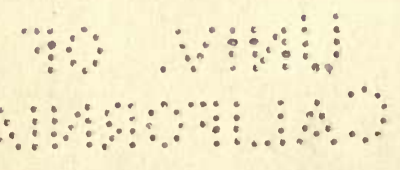




\section{NOTE}

I HAVE avoided the overloading of this volume with references to authorities, but my debt to many contemporary writers will be sufficiently clear to the reader. I must, however, express my very special obligations to my colleagues, Dr. A. Wolf and Dr. T. Percy Nunn, both of whom carefully criticised the manuscript and suggested many important modifications. Dr. Wolf has also read the whole of the proofs, and Dr. Nunn has given me an expert's aid in the chapter in which I have had to refer to mathematical method. Neither of my friendly critics, however, are responsible for the final form of the text, and any errors or failings that are detected in it must be imputed to myself.

$$
\text { L. T. H. }
$$


(4) 


\section{CONTENTS}

INTRODUCTION - $-\quad-\quad-\quad-\quad-$ P. xv

\section{PART I \\ THE LINES OF DEVELOPMENT}

\section{CHAPTER I}

THE NATURE AND SIGNIFICANCE OF MENTAL EVOLUTION

(1) The biological view regards Mind as an organ evolved to adapt behaviour to the environment, (2) and tends to reduce its action to a mechanical process. (3) Parallelism in the end reduces Mind to an epi-phenomenon. (4) The object of Comparative Psychology is to determine empirically the actual function of Mind in successive stages of development. (5) It involves a social as well as an individual psychology. (6) The statement of the higher phases also opens up philosophical questions, (7) and on the solution of these depends the final interpretation of the recorded movement.

Pp. 1-17

\section{CHAPTER II}

THE STRUCTURE OF MIND

(1) Mental operations are known in the first instance as objects of consciousness. (2) Mind is the permanent unity including consciousness and the sum of processes continuous with consciousness and determining it. / (3) These processes involve, but are not identical with, physical processes, constituting with them a psychophysical unity. 


\section{CHAPTER III}

\section{THE GENERAL FUNCTION OF MIND AND BRAIN}

(I) The generic function of Mind, as of the nervous system, is correlation. (2) The special organ for effecting fresh correlation is consciousness. (3) The deliverances of consciousness arise from stimuli acting upon structures built up by experience, (4) on foundations laid by heredity, (5) which supplies not only specific adaptations, but a background to the entire life of consciousness.

$$
\text { Pp. 29-40 }
$$

\section{CHAPTER IV}

\section{MENTAL DEVELOPMENT IN THE INDIVIDUAL}

The nature of correlation. Its relation to consciousness, and the means of distinguishing its principal types. Behaviour in general may be (a) uncorrelated, or (b) correlated. The latter falls into three main classes : (I.) Correlation by heredity, including ( 1 ) inherent structural activity, and (2) reflex action. These are modifiable (II.) by correlation by co-existent conditions. Of such correlation we may distinguish: (1) Equilibration, by which is meant the effect of the state in which the organism finds itself upon its action. (2) Sensorimotor action, in which sense stimuli are so correlated as to adapt general tendencies to individual cases. (3) Instinct, in which trains of action are correlated with vital needs. Pp. 41-60

\section{CHAPTER V}

\section{INDIVIDUAL EXPERIENCE AND SOCIAL TRADITION}

Behaviour is further modified (III.) by correlation based on experience. Here the effect of the past experience of the individual is seen : (I) In modified susceptibility to present stimuli. (2) In accommodation of the equilibrium point to accustomed conditions. (3) In the correlation of action with the relations of objects. The lowest stage of this correlation is Inarticulate, and has two forms responsible for the acquisition of skill and the formation of habit. (4) There follows the Articulate correlation of concrete elements, involving the evolution of ideas, (5) the correlation of universals, and (6) finally the correlation of the principles underlying the activity of correlation itself. On this basis the entire process of evolution may be brought within the conscious sphere. Pp. 6I-89 


\section{CHAPTER VI}

\section{THE EMPIRICAL ORDER}

The development of the last two stages falls within the limits of social evolution, and breaks up into subordinate phases.

(I) In tracing these we begin with the consideration of the empirical order. (2) But there is an earlier stage, of which we find survivals, in which this order is not formed. This stage is char-. acterised : (a) by the absence of the common sense categories; $(b)$ by the dependence of belief on feeling. (3) The empirical order, the characteristic product of the second stage, does not exhaust reality. (4) Consequently another order is built up, (5) which comes to be contrasted with the empirical as the supernatural.

Pp. 90-107

\section{CHAPTER VII}

\section{THE TWO ORDERS}

(1) The limitations of the empirical order are the natural result of development under the conditions of survival. (2) This statement assumes a view of reality, which will require justification later. (3) But we have first to trace the history of reconstruction, which has two main phases-the conceptual and experiential -and two main motives-the religious and scientific. (4) The religious reconstruction is dominated by the needs of man, and issues in the formation of a higher spiritual order, which is either set over against the empirical, as in popular monotheism, (5) or seeks to absorb it, as in Oriental mysticism ; (6) but in either case practically confronts man with two orders, which he has to adjust as best he can.

- Pp. 108-121

\section{CHAPTER VIII}

\section{CONCEPTUAL RECONSTRUCTON}

(I) The main work of Greek philosophy and science lay less in the interrogation of specific experience than in the elaboration of a conceptual order. (2) A conceptual order is constructed without specific reference to experience by analysis and synthesis, (3) and depends for its vitality upon certain principles governing the legitimate use of these operations. (4) When the concept is taken apart from experience, metaphysical difficulties arise. (5) Prominent among these are : first, the formation of unreal abstractions, and secondly, a distorted view of experience as seen, e.g. in the tendency 
of analysis to break up continuity and dissolve organic unity. (6) These defects do not prove any inherent vice in analytic methods, but show the necessity of a critical account of their function in relation to experience. - _ - Pp. 122-149

\section{CHAPTER IX}

\section{EXPERIENTIAL RECONSTRUCTION}

(1) Modern philosophy aims at a reconstruction of experience, (2) first by an evaluation of the subjective factor in knowledge, (3) and secondly by constructing a logic of experience. (4) The problem of knowledge is set in terms of a synthesis of experience, (5) in which the idea of development has a central place. (6) Reconstruction has brought the results of thought into relation with the underlying conditions of its development, and thus rendered Mind self-conscious in the full sense. - Pp. 150-168

\section{CHAPTER X}

\section{THE WILL IN DEVELOPMENT}

The ethical order involves a system of related elements which have to be examined in their development. ( 1 ) There is a hereditary basis of behaviour, roughly adapted to the needs of personal and social life. (2) Hence arises the equally rough adaptation of the feelings which, at the "Inarticulate" stage begin to modify impulse. (3) With "Articulate" correlation there arise desire, choice, individual relationships, and susceptibility to praise and blame. (4) General characteristics of human morality, rules of conduct, social and psychological implications.

The principal stages to be distinguished in the development of human ethics are: (1) Custom, in which the impartial moral judgment is imperfectly formed. (2) Law and morals, in which categorical rules are established. (3) Religious idealism, which sets up a complete system of life, without regard to the actual conditions of development. (4) Realism, which reconstructs the system by relating it to the final meaning of development. (5) A rational account of morals was first attempted by the Greeks, but did not in their case start from a generally accepted spiritual feligion. Such a religion formed the starting point of the modern reconstruction, which has to reconcile the claims of the spiritual order on the individual conscience with the working life of society. (7) This leads to the conception of the harmonious development of man in society as the basis of the ethical order, $(8)$ and to the conception of the spiritual as the moving force in this development. 


\section{CHAPTER XI}

\section{SOCIAL DEVELOPMENTS}

(1) The development of thought is roughly co-ordinate with that of social life in general. On all sides a certain net movement in the same direction may be verified. (2) The control of the physical environment passes through four stages, closely comparable with those of the movement of thought, (3) and the same parallelism can be traced in ethics and religion, (4) and, with exceptional irregularities, in art. (5) Political development corresponds to the ethical in net result though not in all intermediate stages, (6) and a corresponding net movement may be traced in the main departments of law and custom. (7) Progress is irregular because its conditions do not harmonise until a high stage is reached, and because it proceeds from many independent centres. - - Pp. 204-230

\section{PART II}

\section{THE CONDITIONS OF DEVELOPMENT}

\section{CHAPTER I}

\section{EXPERIENCE AND REALITY}

(I) To determine the significance of the development traced in Part I., we have first to examine the validity of the theoretical and practical order reached in the highest phase. (2) Arguments derived from the nature of knowledge do not prove that reality is unknowable. (3) There is no contradiction inherent in the partial interpretation of reality by the correlation of experience, though the categories which are used may require re-modelling when applied to reality as a whole; (4) nor do the alleged limitations of analysis provide any valid argument against the rational method.

Pp. 233-249

\section{CHAPTER II}

\section{THE VALIDITY OF SCIENTIFIC RECONSTRUCTION}

(I) We have still to establish positively the validity of scientific reconstruction. (2) The hypothetical method is logically imperfect, and tends to resolve itself into a descriptive method. (3) Yet science is not mere description. (4) Valid generalisations are ultimately based on a single principle, (5) which is a rule of thought, 
expressing the common principle of all acts of inference, so far as they are mutually consistent. (6) Rationality is the impulse to the formation of a system of parts, which are mutually necessary, (7) and practical rationality is the impulse to the establishment of a similar system in the world of feeling. - _ _ Pp. 250-280

\section{CHAPTER III}

\section{THE PAST AND THE FUTURE}

(I) Rational development is founded upon the Real order, but to estimate its future we must examine its conditions. (2) The general condition is that harmony is a cause of success, (3) while the extension of conscious correlation is the condition of complete harmony. (4) But we cannot tell how far harmony can be developed unless we can determine whether Mind is a true cause of growth, and how it is related to other causes affecting the life of man. This necessitates an inquiry into the nature of causation.

Pp. 281-294

\section{CHAPTER IV}

\section{MECHANISM AND TELEOLOGY}

(I) To explain a thing may be to refer it (teleologically) to its place in a system which, as a whole, has value, (2) or (mechanically) to its immediate antecedent in indifference to any system. The full explanation of a machine involves both kinds of explanation. In a machine, the system pre-supposes an external agent. In an organic whole the systematic principle is inherent in the nature of the parts. (4) The living being is comparable to a machine, devised with the purpose of self-maintenance, (5) but when closely examined, is seen to diverge from this type in that the action of the parts is constantly adapted to the needs of the whole. (6) Where this adaptation proceeds from the nature of the parts as such, i.e. where the action of each part is conditioned by its effect on the whole, we have a true organism. (7) An organic whole is therefore like a machine in being purposive, though unlike it in that its purpose is within. (8) A purposive process is one determined by its tendency to produce a certain result, (9) purpose itself being an act determined in its character by that which it tends to bring about. As such it differs fundamentally from a mechanical cause. (10) Ultimately all purposive systems are of the organic type. How far we may explain collocations by referring them to such systems has still to be discussed, (I I) but on inductive grounds, we must take intelligent action as truly purposive, and not resolvable into mechanical laws. (12) In a less developed form the teleological principle explains all types of genuine organic activity. 


\section{CHAPTER V}

\section{EVOLUTION AND TELEOLOGY}

(1) Light may be thrown on causation by the principles implied in scientific proof. (2) The most general of these is the impulse to conceive reality as a self-explaining whole-that is, one that has an inherent reason for its character. The only known inherent reason is a value. (3) The value, however, must be conditioned by the elements which it also conditions. (4) This argument assumes that a complete system can be conceived, which is doubtful. Arguing from the ideal of thought, we can prove only that, so far as reality is fully intelligible, it must be a teleological process; (5) but arguing from the principle which underlies mechanism, that all variable relations must be resolvable ultimately into relations holding between the terms as such, (6) we can show that variable relations must belong to a whole, the elements of which necessitate one another at some point of time. (7) Such mutual necessitation must run through all that is real, and constitutes a harmony, or self-maintaining system. Since such harmony does not exist and could not have vanished if it had existed, it must lie in the future, and the actual order of reality is determined teleologically by the impulse to realise it. -

- Pp. 331-351

\section{CHAPTER VI}

\section{DEVELOPMENT AND HARMONY}

(1) Development in general means extension of organic unity. (2) It involves a re-arrangement of forces, which, at the outset, cancel each other, first into structures and then into organisms. (3) The formation of organisms depends on the liberation of forces previously cancelled by mutual inter-action, or on the coming together of forces that can act in harmony. (4) Development then proceeds through repeated synthesis towards the universal harmony, which is the final aim of the whole process. (5) The origin of Mind in the individual must be regarded, like other developments, as a synthesis of elements which separately are not Mind. (6) The teleological argument also postulates a central Mind, of the relation of which to reality we can as yet give no adequate account. It must, however, be a conditioned mind. (7) This result corresponds to the actual mingling of good and evil in the world. (8) The empirical and philosophical arguments point to the same conclusion, that reality is the process of the development of Mind. 



\section{INTRODUCTION}

THE field covered in this volume is wide and the treatment in many parts is necessarily short and summary. In justification it must be said here that the book completes a scheme which has occupied the writer for twenty-six years and has been carried through successive stages in three previous works. But in the meantime it was inevitable that the scheme itself should change and expand, and the precise aim of this final instalment will therefore be most readily explained by giving a slight account of the manner in which the subject developed in the writer's mind during the somewhat extended period in question.

In the middle of the "Eighties," when the writer was first studying philosophy, the biological theory of evolution was already very generally accepted, and the philosophical extension of the theory by Mr. Herbert Spencer was, except in academic circles, in the heyday of its influence. Philosophically Mr. Spencer was not a materialist. But his metaphysical safeguards did not rescue the evolution theory from some of the most unfortunate consequences of a materialistic system. Evolution, as thus interpreted, meant, in its bearing on human life and action, essentially two things. It meant that the human mind must be regarded as an organ like the lungs or the liver evolved in the struggle for existence with the function of adjusting the behaviour of the organism to its environment. It was 
to be thought of (the conception is set out more fully in Chapter I.) as a sort of glorified reflex action. Cunningly constructed as it was, it had no special significance in the evolutionary scheme, and though it made man for a time the dominant animal, yet the ultimate goal of its efforts would be to establish an equilibrium which would prove, as Mr. Spencer candidly admitted, the first stage of decay. The Genus Homo had its place in geological time like other genera, and like them would pass away, only unlike them its fossil remains would never become a theme for the antiquary, because in the cooling of the earth there would be no antiquarians. The teeming life of the world must gradually disappear and give place in time to the primordial silence.

The appearance of an upward process in evolution then was illusory. It was due to the position of the human observer, who could not clearly see beyond the segment of the whole curve on which he himself happened to be placed. This result was more fatally apparent when the conditions of evolution were taken into account, and these bring us to the second point at which the theory affected human life and action. So far as there was anything like progress, it was due to the internecine struggle for existence. But a little reflection suffices to show that if progress means anything which human beings can value or desire, it depends on the suppression of the struggle for existence, and the substitution in one form or another of social co-operation. There was here a conflict between the scientific and the ethical points of view which threatened social ethics with extinction. The contradiction was masked indeed for $\mathrm{Mr}$. Spencer by his theory of the inheritance of acquired qualities, and it was not until Weismann insisted on the all-sufficiency of natural selection that it assumed its extremer form. But the social implications of natural 
selection were already apparent before Weismann's work acquired its ascendency, and were so far accepted by Mr. Spencer as to be made the basis of an uncompromising economic individualism. This assertion of individualism coincided with the beginnings of a new demand for the extension of collective responsibility and the social control of industrial life. Economically the old individualism was dying, and apart from the evolutionist school, it was clear to thinking men that the idea of liberty required a new definition. Such a definition was propounded by T. H. Green, whose influence, together with that of the late Master of Balliol, was dominant in Oxford and in the English and Scottish Universities generally in the Eighties and early Nineties. In this philosophy there seemed to many to be a way of escape not only from a barren individualism but from the whole philosophy of evolutionism. An adaptation of German metaphysics, a modified Hegelianism, or a form of Kantianism in which what was best in the Hegelian criticism was incorporated, might maintain itself against science and justify a spiritual conception of human life and of the entire world order. This method however, to speak frankly and quite personally, I could never accept. Apart from all difficulties of detail, two things always seemed clear to me. One was that the attempt to regard reality as all spiritual was as fatal to clear thinking and to the most cherished ideas of the Idealist himself as Materialism. When everything is spiritual the spiritual loses all distinctive significance, and none of the shifts by which Idealism explains error and evil have ever seemed to me to turn or even to approach the central difficulty. My second conviction was that the philosophy of the future must make its account with science. Whatever the limitations of scientific method and the faults and even the blindness of scientific specialism, the plan of building from the 
foundations of experience, the principle of working piecemeal and admitting the broken and incomplete character of knowledge was and is for me sounder than the method of constructing a complete and rounded system at a stroke by some brilliant, perhaps too brilliant, piece of analysis. Metaphysical analysis clearly had its function in setting out and co-ordinating the underlying ideas of science and of experience generally. But I could never accept the view that the whole work of science was of secondary importance, that it could go on constructing its world as it chose, but that whatever its results, a metaphysical analysis would always be able to reinterpret the entire scientific scheme on its own lines. Doubtless metaphysical analysis and scientific specialism has each its sphere, but they cannot maintain an attitude of mutual indifference to the end. Neither is all-embracing, and a true philosophy, a really concrete interpretation of our experience as a whole, must aim rather at a synthesis in which the analysis of first principles figures as the keystone of the arch of science. In this respect $\mathrm{Mr}$. Spencer, whatever the defects of his method, seemed to me to have been justly inspired. But for a long time I did not imagine the function of philosophical criticism to be anything but critical and negative. It was not till much later that I came to think that it might yield certain sound generalisations as to the nature of reality, and I confess I should not even now attach more than a speculative importance to such generalisations if they were not corroborated by a synthetic view of experience.

In the meanwhile I was convinced that a philosophy that was to possess more than a speculative interest must rest on a synthesis of experience as interpreted by science, and that to such a synthesis the general conception of evolution offered a key. The immediate question was whether it was possible to overcome the contradictions of that theory as 
applied to human progress. At this point, philosophical criticism offered a suggestion. The idealistic writers continually insisted on the special features which distinguish human consciousness, and as the later development of psychology has shown, many of their contentions were empirically sound, even if they could not carry the whole weight of the metaphysical superstructure placed on them. Green's permanent self-consciousness, for example, if it is not a spiritual principle, eternal or timeless, is an empirical fact within the world of time. It was the temptation of an empirical, and in particular of an evolutionary psychology, to explain away these higher developments of mind, to level distinctions of kind, and so reduce all mental phenomena as nearly as might be to the same level. This, I thought, might be the root of the trouble, and I conceived that if the mental or spiritual side of evolution were treated quite dispassionately, without any attempt to minimise differences of kind, but setting them out impartially and using them to measure the length of line which by whatever means evolution had somehow traced out, a very different interpretation of the whole process might be reached. As I followed this line of thought, it seemed to me that, details apart, the Hegelian conception of development possessed a certain rough, empirical value. There were grades or degrees of consciousness and self-consciousness, and as personal self-consciousness was distinctive of man, so there was a higher self-consciousness of the human spirit, which would represent the term of the present stage in development. Further, if this conception was interpreted in terms of experience, it indicated a point of union, where one would not expect to find it, between the Idealistic and the Positivist philosophy. This higher self-consciousness would be the Humanity of Positivism, regulating its own life and controlling its own development. But further, if 
this was the true empirical account of Evolution, our interpretation of that process would be fundamentally changed. The factor of consciousness, as the late Professor Ritchie was already insisting, would influence the course of development. If my view was right it would turn out even to be the central point in development. To the fully conscious mind in man everything would lead up, and from it, once formed, all future movement would be derived. This was indeed to assume that along with knowledge there would go control, but in the first place it could, I thought, be shown that control extends in a kind of geometrical ratio with each new turn in the development of consciousness, and in the second place, as the full meaning of the self-conscious mind worked itself out it was seen to imply a grip on those underlying conditions of life which, as long as they remain obscure, thwart human effort and distract man from that social collaboration which is necessary to the greatest efforts.

By emphasizing consciousness and its control moreover, several difficulties as to the relations of evolution and progress could be met. To begin with, it was possible to conceive of evolution in general as a blind and even brutal process, dependent on the anarchical struggle for existence, but to maintain that in the course of this struggle there had arisen among other species one which owed its survival to a mind. How this had happened was not for the moment the point. It had happened, and there was a being with a mind, looking before and after, and also looking around him upon his fellows and on the whole, working with as well as against them. Something of this mind moreover existed in lower species, and it was important to notice that even there, in proportion as mind began to exert itself, it tended to supersede the struggle for existence. It was possible to display one particular line of evolution, for 
which I afterwards found Mr. Sutherland's expression "orthogenic evolution" as a series of advances in the development of mind involving a parallel curtailment of the sphere of natural selection. The conclusion was clear that natural selection was not the cause of progress, if progress meant the advance of mind. But what was the cause of progress, how mind came into being, how it grew, what were the conditions of its further development, I did not at first enquire. I saw no light upon the question, and I thought that the empirical account leading up as it did to the control of life as a whole by consciousness was the most important or at least the first thing to prove.

There was further difficulty with regard to human progress which could be met by emphasizing this factor. If it was admitted that man was something higher than the animals, it might easily be denied that modern man was anything higher than ancient man. Certainly if we take specially favoured races and epochs of the past for comparison, there is not the slightest proof of any advance in average human faculty. True, social progress does not necessarily require any improvement in the congenital qualities of the individual, and the question should be rather whether the collective achievement of mankind grows-in knowledge, religion, ethics, art, social organisation. But on all these points, with the exception of knowledge and its direct applications to industry, scepticism is abundantly possible, and it is easy to assert that there have been earlier epochs when religion was purer, social life better organised, men and women on the whole happier, and industry devoted to the production of more beautiful objects than sky-scrapers, factory chimneys, gigantic hoardings and aniline dyes. I was never one of those who think that the general fact of progress may be readily assumed, or that mankind constantly advances to higher things by 
an automatic law which can be left to itself. On the contrary, I believed that there was no upward tendency in things as such, that apart from the operations of the human mind, the struggle for existence ruled, that the sun of its favour shone impartially on the just and the unjust, and the east wind of its implacable severity nipped the buds of loveliest promise as readily as the garden weeds. Not only so, but until the mind should come into its kingdom man himself was subject to the same rule. The struggle for existence was not the cause of mind, but mind had to undergo the struggle for existence. Each animal species that relied on a dawning intelligence for its living had to maintain itself against others that might be harder of shell or stouter of limb. Each race of man that made some advance in ideas, in industry or the social arts had to fight for its place. There was no a priori reason to suppose that it would survive. Its mental development would be on the whole an advantage, but it would only be one advantage among many possibilities, and a higher birth-rate, a tougher hide, stouter muscles, or greater power of resistance to some microbe might easily turn the scale of any conflict in favour of a rival race of lower mental endowment. It was therefore clearly possible, and the historical record showed that it was the fact, that the higher type may often be beaten by the lower, and beaten to extinction so far as its achievements in civilisation are concerned. Only if mind should once reach the point at which it could control all the conditions of its life, could this danger be permanently averted. Now it seemed to me that it is precisely on this line that modern civilisation has made its chief advance, that through science it is beginning to control the physical conditions of life, and that on the side of ethics and religion it is forming those ideas of the unity of the race, and of the subordination of law, morals and social 
constitutions generally to the needs of human development which are the conditions of the control that is required. It seemed of secondary importance that there should have been little or no progress in other respects, provided that this essential condition of future advance had been realised.

The first object then, as it seemed to me, was to show that mental evolution had in point of fact consisted in a development of consciousness from stage to stage in the manner supposed. To do this would require a very wide examination on the one hand of animal psychology, on the other of the growth of human thought and of the social customs and traditions in which thought is embodied. But there were also problems of definition and analysis. Consciousness and self-consciousness are vague terms. If we are to distinguish phases of their growth accurate criteria are required, and the criteria should be such as are directly reflected in external behaviour. For in the case of animals we have nothing but external behaviour to go by. In the case of man our judgment has to be in large measure indirect, based on the implications of a custom or a belief, or even a phrase. In all cases it was an integral part of the purpose to determine not merely what consciousness was but what it effected. For these reasons I came to take the correlation which is effected in consciousness between different portions of our experience or between different acts and purposes as the basis of a classification. The starting point of this conception is exceedingly simple. If we utter a simple sentence we bring different words, and the words stand for ideas or elements of ideas, into relation. If we execute a purpose we bring a series of acts into relation with one another. It is by correlation that the mind introduces order and establishes its control. There is, however, in organic life a certain degree of correlation apparently independent of consciousness. Thus the several 
organs of the body act on the whole in concert, or, to take an instance of another kind, the successive operations of an instinct, e.g. the spinning of a spider's web are nicely correlated with one another, though we cannot assume that this adjustment is effected by intelligence. The term correlation therefore serves, first, as a summum genus under which all kinds of vital activity, conscious or unconscious, might be subsumed, and secondly, as a standard by which they might be compared, certain assignable differences in the method and scope of correlation yielding the required differences of type which are successively evolved. There was here a standard measure for the evolution of mind, and to carry it right through that evolution has been the principal task. It was worked out in some detail for animal psychology and for the transition to human faculty in Mind in Evolution, published in rgor. For human evolution the ethical side seemed most important, and this was worked out in Morals in Evolution five years later. The data are in all cases difficult to ascertain with precision, and the analysis has required constant overhauling and restatement. ${ }^{1}$ The results are summarised, modified, and

${ }^{1}$ Animal Psychology had barely emerged as a science twelve years ago, and there was little then to rely upon beyond the pioneer work of Romanes and the judicious observations and careful reflections of Mr. Lloyd Morgan. Mr. Thorndike's experiments, however, had laid the foundations of a new method, which has been brilliantly developed by a series of American observers and experimentalists such as Profs. Yerkes, Jennings, Haggerty, Watson, Shepherd and many others. Animal Psychology as it stands may fairly be considered the creation of American science. I regret that owing to the extended field covered in this book I am unable to deal worthily at present with this new wealth of material, but it has naturally modified my old opinions on several points, as is briefly indicated in its place.

In comparative ethics again to the work of Post, Letourneau and Sutherland, which were the best available surveys ten years ago, should now be added the encyclopaedic researches of Dr. Westermarck, and it is hardly too much to say that this subject has also definitely entered the rank of the sciences. 
extended in the first part of the present work, the object of which is to state as definitely as possible what is involved in the evolution of self-conscious mind, and to show that this evolution has in fact proceeded by successive stages from the dawn of life to the rise of modern civilised thought,

In all this part of the work the method was rigidly empirical, or to use a descriptive, though not very desirable term, phenomenological. In fact in the two earlier works mentioned I confined myself almost entirely to a comparison of the actual content of each stage in development, avoiding theories of the nature of life and mind, and current controversies as to causation. The account should, I thought, hold true whether mental process should ultimately be resolvable into mechanical terms or not. It should also be independent of any theory of the ultimate nature of reality. There might or might not be an original purpose in things, but there was certainly an evolved purpose, and this purpose at its highest point of development $\angle$ would acquire a superhuman, a quasi-divine power. The genesis of this power could, I thought, be verified in experience, and that was a more solid ground than any metaphysical analysis. In point of fact I was at first opposed to anything like a theistic or teleological interpretation of reality as a whole, as inconsistent with the mechanical causation which I took to be the ultimate category of science.

There are, however, elements of fallacy in the purely empirical view, or at least in the inferences which I drew from it, which are set out here in Part II. Chap. III. and the sense of this deficiency compelled me to take further account of the questions of causation which had previously been set aside. For this examination there was a starting point in some results which I had reached in following up another line of enquiry. To justify the empirical method 
it was necessary to examine the foundations of knowledge, in order to discover whether the postulates of the empirical view were self-consistent and self-sufficient. For this purpose, before beginning the systematic study of evolution several years were given to an examination of the Theory of Knowledge (1896). Working with the ideas of mechanical causation in this book, I was led to the conclusion that these ideas themselves imply at the end what might be called an organic conception of reality as a whole. But the organic seemed to me then as distinct from the purposive on the one hand as from the mechanical on the other. Not long after the book was published, however, some new considerations occurred which convinced me that this was an error, and that however much I might object to the form of their reasoning there was an element of substantial truth on this head in the reasoning of the Idealists. The result was to suggest that by mechanical reasoning from a purely empirical starting point a candid thinker would be led to admit an element of purpose in the system of Reality. It thus became important to connect this result with the empirical account of the growth of purpose.

This is the principal object of the present work, and the result, if the reasoning is sound, is to show a coincidence between the views derived from an analysis of the presuppositions of knowledge, and those attained by a comprehensive review of experience. The analysis suggests the operation of a conditioned purpose. The empirical account reveals the purpose in operation. Many difficulties remain which will be found freely admitted in the text, but it is submitted, not in the least as a matter of faith, but as a sound working hypothesis, that the evolutionary process can be best understood as the effect of a purpose slowly working itself out under limiting conditions which it brings successively under control. This would imply not 
that reality is Spiritual or the creation of an unconditioned mind - a view equally repugnant to morality and experience -but that there is a spiritual element integral to the structure and movement of Reality, and that evolution is the process by which this principle makes itself master of the residual conditions which at first dominate its life and thwart its efforts. It is of course true that the evolution whose story we know is confined to a single planet, but it is argued that this terrestrial evolution coincides in outline with the conclusions of an analysis that is applicable to reality in general. For further verification we must be content to await further enquiry.

The relation between the 'historical' and the philosophical argument will be further considered in Chap. I., but one point may be subjoined here. The conception of Mind and its evolution differs fundamentally in accordance with the position given to the rational element. Now in the history of philosophy it was the rational that first interested thinkers. They wished to know what was reasonable and why, both in thought and in conduct. Often, no doubt, they were led to speak as though thought were, and action ought to be, purely rational, and they neglected the study of the elements of impulse, instinct, feeling, emotion that made up the groundwork of human psychology. In recent years the pendulum has swung the other way. The irrational is the chief object of interest, one may almost say of adulation. Indeed it becomes almost difficult to get a hearing for any theory which still regards reason as a good name for that which distinguishes man from the lower animals. Everyone takes a pride in showing his superiority to mere reasoning, and there are some who are at least successful in demonstrating their freedom from any bias in favour of rational methods. The causes of this curious reaction would repay an investigation 
for which unfortunately there is not sufficient space here. In part it is due to the more concrete study of psychology and the prominence which any science, particularly in its more popular versions, is apt to give to newly opened territory. In part again it arises from the extraordinary discoveries of science itself, which have undoubtedly undermined many old categories, and seem to some to have made almost anything possible. Another factor is the old desire to be free of rational trammels, and create in imagination a world which will satisfy the cravings of man-a desire which in these days fortifies itself with odds and ends from the psychology of faith-healing. For if faith can remove blisters, why should it not remove mountains? All this reaction is of purely temporary significance. Rational purpose is, and will always in the end be, recognised as the distinctive feature of the activity of mind, and though it may fairly enough be maintained that the mind is more than its purposes, and that the purposes themselves grow and take definite shape in the very process of execution, this is only to contend that the mind, as we know it, is still imperfectly aware of its self and its own meanings. It is to set one problem the more to the student of the evolution of self-consciousness. A mere vital impulse may blow like the wind where it listeth, so that none can tell whence it cometh or whither it goeth. But creative or rather plastic mind is that which moves towards ends which are worth reaching, and because they are worth reaching. It gets a better view of them as it advances, not so much because they are nearer as because its own nature as mind is being all along developed by its activity and its experience, and this development means precisely that its purposes are clearer, more harmonious and more comprehensive.

To justify this view of mind it has to be shown that the postulates of logical thought are intelligible and self- 
consistent, that they form indeed a rational system. In the same way it is necessary to show in ethics not merely that there is a certain order which as a matter of fact is coming to prevail, but that there is a rational order. This task is attempted in the earlier chapters of Part II., and the evolution of mind is conceived accordingly as a progressive development of the rational both in thought and in action. The conception formed of rationality proves in fact to be the connecting link between the historical account of mental evolution and the philosophical theory of the ultimate basis and meaning of evolution. Three studies are thus closely linked, the history of mind in living beings, the validity of its rational processes, the position of mind in the structure of reality as a whole. In the position here adopted, the conception of reason is no doubt considerably widened. Neither in logic nor in ethics is the rational function confined to the apprehension or application of certain abstract ideas. It is conceived rather as a principle of harmony pervading experience and working it into an organic whole. So understood, reason is supreme in the mind simply as that which embraces every element of experience, interconnects every feeling and thought, takes account impartially of every suggestion and every impulse, and weaves of them all a tissue which is never ossified but always plastic and recipient. It is the conscious expression of that impulse to harmony which dominates the entire evolution of Mind, and the rationality of the process is the guarantee of its ultimate success. 



\section{PART I}

\section{THE LINES OF DEVELOPMENT}





\section{CHAPTER I}

\section{THE NATURE AND SIGNIFICANCE OF MENTAL EVOLUTION}

I. IN the biological theory of evolution the development of mind takes a secondary place. The biologist is concerned with the laws of variation and heredity. As an evolutionist, his main interest lies in showing that certain known facts of variation and certain established laws of heredity suffice to explain the development of the existing forms of flora and fauna in all their wealth from a single primitive type. A parent organism, an original living being he has for the present to assume. Recent physico-chemical research has indeed strongly suggested that the evolutionary principle extends beyond the living world, that the specific forms or 'elements,' as we still call them, of 'inanimate matter' may be conceived as developing in geologic time from a simpler, perhaps from a single primordial type, and that this type would be something (if the expression be allowed) not strictly material, but rather pre-material. But the gulf between the living and the inanimate remains for the present un-spanned. The biologist has to assume the existence of living tissue, just as the physicist has to take the existence of negative and positive electrons as a datum which he does not seek to explain. Granted the existence of the living germ, however, the biologist can do much towards explaining the derivation from this single source of the vast complexity of forms which actually people the world. Not that in 'explaining' he pretends to give the ultimate reason for all that he finds. Ultimate reasons are not precisely the concern of a special science. He explains 
or seeks to explain in the sense that he traces the whole movement of organic life and the vast complexity of organic forms to the operation of a few clearly established empirical laws. The first of these laws is that all living beings in the normal course of their life-history give rise once or oftener to other living beings by separating off a portion of their own tissue. The second is the equally familiar fact that the new living beings, either directly, as in the case of cell division, or after a number of cellular generations, as in the case of sexual reproduction, come to resemble their parent or parents in general type. The third fact is that this resemblance is not absolute, but is qualified by a certain degree of individual variation. The fourth is that under some conditions such variations are in turn perpetuated by heredity. The fifth is that of many individuals born only a certain proportion-among the lower organic types only a very small proportion-come to maturity and so reproduce their species in turn. To these may be added a sixth and last fact, that every living being is born into an environment in which it has to maintain itself against dangers and provide itself with the necessaries of life.

These are for the most part very simple statements of almost obvious fact. Yet in the hands of biological science these very simple considerations go far to explain the labyrinthine complexities of the actual development of life on the earth. It is true that when we come to close quarters certain of these statements raise questions of controversy which are by no means so simple. What, for example, are the nature and limits of that variation around the parental type which manifestly forms the point of departure for the entire process? Are all variations quite small and delicately graded so that there is always a continuity between any given type and any other that we recognise as related but distinct? For a generation after the publication of the Origin of Species it was the ambition of biologists to reduce all changes of form to variations of this kind, and so exhibit evolution as a continuous process. In later years, however, experiment seems clearly to have shown that, explain them as we may, wider varia- 
tions also occur, and new varieties, if not new species, come into existence at a birth. Further evidence has gone to show that these variations, to which the name of ' mutations' has been given, are perpetuated by heredity, while the permanence of the smaller 'fluctuations' has become in some degree matter of doubt. But whether 'mutations' or 'fluctuations' are the more important in the history of organic evolution is a question which does not affect the validity of the propositions above laid down, nor impair the conclusion drawn from them. Mutations are still variations in individuals qualifying the general resemblance of the parent stock, though they are variations of a different order from that contemplated when individual variations were first conceived as the starting-point of new species. What is still more important, their perpetuation is subject to the conditions of the environment. If the mutation is such as to unfit its possessor to cope with the conditions of life he will not survive to maturity. $\mathrm{He}$ will not reproduce his type, and the mutation will disappear. If, on the other hand, the mutation is favourable, the stock once gifted with it will multiply and possess the earth.

In fact the very simple but far-reaching theory which the biologists derived from the still more simple propositions laid down above is not seriously affected by newer views of heredity. Put into its simplest terms the theory merely combines the statements of fact/laid down above and draws out the consequences. Variations arise-no matter how. We keep to the bare fact. Arising, they are perpetuated-again by what mechanism of heredity does not for the moment matter. But they are perpetuated only if they assist, or at lowest do not hinder the individual in the struggle for existence. Hence arises a differentiation of type. Of the varying individuals some are weeded out: others survive. From these in turn new variations proceed to meet with the same fate, or rather the same alternatives of fate. Hence by the repeated formation of fresh centres of variation arise the successive growths which constitute new species, new genera, new orders. 
The one condition which every successful variation must fulfil is that it should assist its possessors in maintaining their own existence, and in engendering and bringing up young ones after their kind. From this point of view the evolutionist expects to find in every new variation of structure some closer adaptation to the requirements of the living species. But in the animal world, particularly as we ascend the scale, there comes into being one structure which in a sense dominates all the remainder. The central nervous system governs the whole body and therewith determines the use to which every part of it is put. But the growth of the nervous system and the entirety of its functions remains for the biological observer merely the most complex and finely adjusted of all adaptations. It is that structure which by the infinite delicacy of its adjustment to the minutest variation of stimulus enables the organism to accommodate itself to a myriad of changes in the outer world, and even to learn from the past and provide for the future. If an object approaches the eyes they blink and so protect themselves. If nevertheless a fragment lodges in the eye a tear rolls down from the lacrimal gland and helps to wash it out. These are very simple instances of reflex adaptation, and they are referred by the biological interpreter to a physical machinery which can in part be traced-viz. to a certain plexus of sensory cells, and nerve fibres, ganglion cells, motor nerve fibres and muscles which make up the regular constituent elements of a reflex act. This machinery is part of the hereditary endowment of the individual. It has come to be, according to the evolutionist's interpretation, because those who could not protect their eyes efficiently lost their sight, and left no descendants, because those who had the best eyes which involved the best protecting mechanism prevailed in the struggle. It is in short the product of a series of adaptations to the requirements of the living organism in its given environment. It is, moreover, interpretable as a purely physical process. The details of that process are still in large measure unknown. But there is no reason to doubt that the luminous waves proceeding from an object and impinging on the rods and 
cones of the retina produce in their cells some physical change. It is known that the change propagates a disturbance along the fibres of the optic nerve and that this disturbance proceeds at a measurable speed in the form of a wave and is accompanied by certain electrical phenomena. The result of this disturbance, to omit the intervening stages, is to set up certain chemical changes in the musclecells which move the eyelids, causing them to thicken and shorten and thereby to draw down the lids. In the case. of the tears the disturbance is communicated to the cells of a gland which it causes, instead of contracting, to secrete their peculiar product.

2. In all this there nowhere appears any reason to impute the existence of any forces but those that we call mechanical or chemical. It is true that the details of the mechanism or of the chemical change are not yet fully made out. But so far as investigation has gone it has yielded no reason for excepting reflex-phenomena from ordinary mechanical laws. The reaction is no doubt complex, but it is pretty nearly as regular and undeviating as the response of any confessed machine to the pressure of a knob or the turning of a handle. The child squeezes its doll and in virtue of a cunningly concealed mechanism it cries. Something squeezes the child and in virtue of a still more cunning mechanism it cries more effectually. There is the mechanical view. And at least in the case of blinking it has this to support it-that the response as a rule is given unconsciously and intelligence neither makes nor meddles with it. The act serves a purpose-yet it is not purposive. It is the result of a preordained structure, of a structure which has come into existence to do that particular thing quite as much as a bit of machinery has. been made to play its particular part whatever it be. It is a case of a function executed by the organism and serving the ends of the organism, which depends nevertheless on purely physical laws and in which conscious purpose has no part to play.

The higher and more complex acts of animals and of man differ, it would be admitted, in important respects from responses of this type. They are not unattended by 
consciousness. To many of them the presence of consciousness appears generally essential. Nor are they uniform and undeviating in their course. On the contrary they are varied from occasion to occasion and even from moment to moment, and varied, it would seem, in accordance with an intelligent appreciation of the needs of the situation. None the less, such examples as those quoted, and the extreme difficulty of definitely formulating any alternative view have suggested an interpretation which would reduce all conscious and therewith all psychical activity to the level of a vastly complicated and glorified mechanism. The series of mechanical changes it is conceived must be unbroken. As the speck of dust sets up a train of molecular movements which ultimately issues in the secretion of a tear, so the stimulus of printed words affecting the optic nerve spreads its wave of influence over the brain and, no doubt through combinations of infinite complexity with other influences, produces by a strictly physical process some final modification in the reader's conduct of life. All that the man so affected is aware of is a series of changes in his own mind-new thoughts, emotional suggestions, the interaction of new and old experiences, the crystallisation ultimately of half-formed suggestions into a new and definite rule of conduct. To him the suggestions appear as the antecedent conditions and his own resolutions as the complete and sufficient cause of the line of conduct that he adopts. But if he propounds this theory to a convinced exponent of mechanical uniformity he is met by some exceedingly difficult questions. The process in question begins with something physical, that is to say with masses in motion, and it ends with something physical; a physical basis, the brain and nervous system, is a necessary condition of its continuance and successful termination. Are we then to understand that there is at some point a break in the physical process? If so, we shall have to say where precisely the break occurs, and this without making arbitrary assumptions we shall have great difficulty in doing. Not only so, but what is more serious, we shall have to assume that at the point where the break occurs a uniform mechanical process by 
which one mass movement gives rise to another in accordance with uniform law comes suddenly to a dead stop. There is some particular movement of some particular particles which sets up no further movement, but instead of so doing has as its effect a modification of a totally different kind of thing called Consciousness. This thing being set to work arrives in its turn at a point where it, not being itself a mass in motion, nevertheless sets a molecular mass in motion, and so presently brings about what we recognise as an act of the individual.

3. This account involves so violent a discontinuity in the causal process that most thinkers shrink from it, and take refuge in some theory of Parallelism. According to this view there is no breach of physical or mechanical continuity. If we could master the whole details of the neural process we should find that in the most complex deliberation, as in the simplest reflex, they run their course in the fixed groove of mechanical law. Motion gives rise to motion within the brain cells and along the brain fibres in strict accordance with the Conservation of Momentum. But certain motions of certain kinds of molecule are for reasons unknown to us accompanied by definite changes in that which we know as consciousness, the relation being so intricately adjusted that there is a point to point correspondence between molecular and conscious modifications. The two streams flow, so to say, not merely side by side, but in one bed. Each, considered internally, exhibits perfect uniformity of sequence, and together they form the whole which is the internal life and external behaviour of the conscious thinking animal.

This theory in turn has many points of difficulty. But what concerns us principally here is to note one of its main consequences. The phenomena of consciousness, metaphysical theories apart, are limited to animal bodies, and moreover to certain processes only which occur within animal bodies. The physical, on the other hand, is everywhere. Thus the process to which a physical stimulus first gives rise and which ultimately issues in a physical action is physical throughout. On the mechanical plane its continuity is unbroken, and its self-determination is 
supposed to be complete. Upon this process the mental or conscious series is at a certain point superimposed, and at another point taken off again. It is as it were a temporary, and so far as the effect of the process is concerned, an accidental and superfluous addition. It is clear from the supposition that the mechanical order must determine itself, and the conscious order take a secondary place. Consciousness in fact becomes what some writers have called it, an Epiphenomenon. So far as the course of events in the universe is concerned, consciousness, feeling; intelligence, forethought, resolution, might as well not be. The secret of organic life is the intricate adaptation of physical structure to respond in such manner as the life requirements of the species dictate to the circumstances of the physical environment.

I shall not for the moment attempt to resolve the difficulties briefly set out. Whether a solution securing a more real position to the conscious factor is ultimately possible will be found to turn in the end on the question whether every event or phase of process must be supposed to proceed uniformly from a pre-existing phase or whether it may be conceived (as we seem to conceive our own efforts) as really determined by relation to that which it itself brings about. With this question we shall deal at length in its turn, and from the discussion some light may I hope be obtained. We may note for the present that the psycho-physical view which reduces the whole mind-life to the rank of an epiphenomenon is merely the most extreme and consistent expression of a result to which the biological treatment of mental evolution tends. 'Mind' is here in all essentials evolved structure. Biologists may be careful to eschew metaphysics and may avoid the charge of materialism by a judicious selection of phrases. None the less it lies in the nature of the biological treatment to think of mental activity like all activity, like muscular contraction or glandular secretion, like respiration or digestion, as the function of a structure. That structure is the cerebro-spinal nervous system, and the functions which that system performs may be summed up in one formula. They are such as to accommodate the actions of the organ- 
ism to the conditions of the environment. They are in man on a very large and complex scale what the respiratory or muscular mechanism is on a smaller scale. As these are arranged to secure a permanent supply of oxygen, the maintenance of an even bodily temperature and so forth, so the nervous system is arranged to secure such action as will, by however circuitous a route, feed, clothe and preserve the organism, cause it to produce children and rear them. The machinery gets more complex, but it is still machinery arranged to secure the ultimate object of the survival of the species. Mind and the world of mind, society, government, the churches, religion, law, are products which have grown up under the pressure of the constant and supreme biological need, and exist only to serve that need. They are evolved to meet the requirements as an aquatic species on taking to the land is held to have evolved lungs, and if their vital function ceases they atrophy as the eyes of a cave-dwelling animal atrophy. Their end and object, their causation, is not in themselves but in the more fundamental biological conditions from which they are thrown up. It must be added that these conditions seem at a vital point to be positively hostile to certain of the effects of mind-development. For it is a general condition of the good adaptation of a species to an environment that the weaker members of the species should be persistently weeded out. 'But with the expan-' sion of mental life come affections and sympathies, and later on religious and ethical sentiments inculcating mutual aid, discouraging the struggle of each for himself and enjoining the preservation of many who but for such assistance would go under in the life-storm. The rise of such sentiments is from the strictly selectionist point of view a case of the emergence of a functionally noxious variation which must be stamped out if the human species is to survive, and the strict spirit of biology has in consequence waged war for a couple of generations on such schemes of social and political amelioration as tend to peace and equity between nations, co-operation between classes, and mercy and tenderness for the weaker brethren. It is however only fair to say that the resulting contradiction between 
the teaching of biology and that of civilisation has at length had its effect and the trend of biological opinion now is to interest itself in the artificial selection of types for reproduction as a civilised substitute for the elimination by natural forces of those who cannot stand on their own feet. With the value of this view, which represents the effects of sociological criticism impinging on a more ruthless doctrine, we are not for the moment concerned. What is of interest is the entire subjugation of the life of mind to biological conditions. It is the survival value of certain types of nerve structures which has given birth to the world of mind, and which remains the condition of further development within that world. Mental vigour, moral worth, as properly estimated, are means by which a type can maintain and improve itself. Whatsoever soul is hard, whatsoever is unlovely, what there is of self-assertion, if there is any ruthlessness, if there is any unimaginative selfcentred push, this type shall prevail, for of such is the process of evolution.

4. If this were indeed so, some might think it better that the process of evolution should cease. But it is worth enquiring afresh whether the account given by biology of the part played by mind in organic evolution is an adequate account. For this purpose it will be necessary to take a summary view of the actual phenomena of mental development so far as they can be ascertained both in animals and in man. This is attempted in the first part of this volume, and it will conduce to clearness if the broad results are briefly stated by way of anticipation.

Our review then will go to show that, without involving any discontinuity either as between the lowest living organism and the intelligent animal, or as between the intelligent animal and man, without, that is to say, involving any change so sudden and great that we cannot conceive it as bridged over by the cumulative effort of small variations, the facts of growth when disinterestedly studied do reveal changes which we ought to regard as changes of kind, and indeed of a kind very material to the interpretation of evolution, of the position of mind in reality, and of the future possibilities of man. That cumulative 
changes of such a kind as are now known to be compatible with the working of heredity may sum themselves up into a distinct change of quality need occasion no surprise. We all know that in the individual bony and muscular tissue alike arise out of an original undifferentiated mass of protoplasm. Yet bone is one thing and muscle another and the protoplasm of a fertilised ovum a third, and if these things are not qualitatively different, the term quality has no meaning. In the same way we shall see grounds for thinking that the reason of man differs in kind from the intelligence of the dog, and the intelligence of the dog differs in kind from the blind gropings of a polyp without proceeding to infer that no course of development could ever have produced the one type from the other. The truth is that it is only when we admit and emphasise qualitative distinctions that we arrive at the full sense of what development means and what it can do. It is the natural tendency of an evolutionary theory in its first phase when struggling for existence to pare away and depreciate the distinctive features of the most highly developed and peculiar structures which it has to explain, to bring them as nearly as it can to the level from which development is to start. This is the natural protective device of an infant theory threatened by enemies in the shape of prejudice and incredulity. The time has gone by when evolutionary theories stood in need of such adventitious and indeed slippery and uncertain aids. We can surely afford now to look the facts steadily in the face and faithfully report the actual scope of mind-development as we find it.

From this study then there emerges as the principal result the recognition of certain qualitative changes which vitally affect our interpretation of the process of human evolution, its genesis, its potentialities and its permanent conditions. The sum and substance of these changes is to effect a complete revolution in the position of mind as it exists in living beings. Coming into existence as the biologist has told us as a means of securing the permanence of the species it never loses that function, and indeed comes to perform it more efficiently. But it ceases to be limited 
by the conditions of its genesis. It becomes self-determining, is guided, that is to say, by values which belong to its own world, and finally it begins to master the very conditions which first engendered it. In the end, when we have fairly taken the measure and grasped the conditions of its growth, we are led to regard the development of mind not as a side product of natural selection but as the central fact of the history of life upon the earth.

5. The development which we have to trace falls into two main divisions. In examining the emergence of intelligence as a factor in the life of the lower organisms, in measuring its growing importance in the behaviour of the higher mammals, and in estimating the qualitative changes which mark the transition from animal to human mentality, we are dealing in the main with the functions or capabilities of the individual mind. But as soon as we begin to follow the track of the higher developments of mind in man the nature of the enquiry changes. The forces to be considered are now social rather than psychological, or, more accurately, are matter of social rather than individual psychology. We have to do not with the emergence of any new faculty, not with any essential change in the structure of the brain or in the sum of hereditary dispositions or capacities, but rather with the social product to which the individual mind contributes its mite, which is gradually built up by millions of individual workmen in the course of ages and which undergoes profound modifications within the limits of recorded history. This branch of our enquiry, that is to say, is concerned with what we may call the social mind, understanding by that term, the Order formed by the operation of mind on mind, incorporated in a social tradition handed on by language and by social institutions. of many kinds, and shaping the ideas and the practice of each new generation that grows up under its shadow. The enquiry into the growth of this tradition is rather sociological than psychological. It is an enquiry into institutions, into creeds, into social relations, rather than an enquiry into the consciousness of individual human beings. The opposition must not be exaggerated. There are 
social forces at work in the psychology of the higher animals which live, some in herds or swarms or flocks, some in families of greater or less permanence. There are also individual and racial differences among men which affect their capacity for supporting or advancing the social tradition, and the question whether actual human faculty-the average equipment with which the individual is furnished by physical heredity-improves with civilisation is an important question. But it is not the main question with which we have to deal in tracing the growth of the social mind. With no change at all in the average hereditary individual capacity in a group, the very greatest changes might be brought about in the course of a few generations by social forces; and the probability is that the greater changes of history, including both the rise and the fall of nations and of civilisations, are attributable to such social causes and not to sudden variations in the average hereditary qualities of races.

Be this as it may, it is to be understood from the outset that the scope of our enquiry includes the social along with the individual. Could it ever be fully carried out it would begin with the most rudimentary germs of mental activity discoverable in the lowest organisms: it would trace the successive stages of mental growth in the higher orders of the animal creation till it reached the beginnings of human intelligence; and thence proceeding essentially by the same method, but concerning itself now for the most part with social forces and social products, it would follow the successive stages in the movement of human thought from its first beginnings to that phase of development in which we live and in which we share. The data for such an enquiry are not and perhaps never will be complete. Our conception of the lowest phases of mind is necessarily inferential, and the path of inference here is surrounded by many pitfalls. Our knowledge of the earlier societies is scanty and at some important points altogether wanting. But in all this we suffer no more than biology suffers from the imperfection of the geological record, and though we may never be able to paint an accurate picture of mental evolution as a whole, there is no reason why we should not 
endeavour to seize on such salient points as may serve to determine its trend and measure the length and direction , of the path along which it has moved.

6. Up to this point, as has been remarked in the Introduction, our method is purely empirical. We have simply to analyse and compare the operations and achievements of mind in successive phases, to show how one phase may be conceived as issuing from another, and to indicate the nature of the changes successively introduced. But particularly as we reach the higher phases we shall see that another set of questions underlies our whole enquiry. When for example we deal with the emergence of rational method, as in science and philosophy, we shall have to take account of the claim of such a method to yield truth. "This claim is an integral part of this particular phase of development, and we shall not be able to understand that phase or place it in due relation to others without enquiring into the nature of rational method and thus opening up the question of the validity of thought. Similarly on the ethical side we shall come upon theories of conduct or of human well-being which we shall not be able to interpret without opening questions as to the meaning of such terms as good and bad, right or wrong. It is true that we might keep to a purely historical method by merely recounting the opinions which men have held or the methods which they have in fact pursued. But it is clear that our conception of a given intellectual movement will differ radically according as we hold that it is a movement towards truth or towards error, or again towards a goal of real value or to one of no greater account than any other. Thus if our object be not merely to record the successive phases in the movement of mind but to appreciate the direction and magnitude of that movement and this is the object which I would propose for the enquiry -it is clear that we have to go outside the purely historical method of treatment ; we must apply a philosophical theory of the basis of rational belief and action in order that we may take stock of the position at which we have arrived. If, for example, we can satisfy ourselves that we have some knowledge of reality, grounded, let us say, on the methods of science, 
then we shall be able to treat the historic development of science as a movement towards the knowledge of reality. If, on the other hand, we take the view that scientificr method suffers from incurable defects or limitations which preclude it from ever supplying a genuine interpretation of the reality of things, then clearly we shall put quite a different valuation upon its growth, and our whole estimate of modern civilisation will be vitally changed. Thus from the study of historical facts we are led on to a study of values, of the ultimate grounds of belief, the meaning of rationality, the possible scope of knowledge, the considerations which reasonably determine action. We have not only to distinguish successive phases of development, but we have to estimate the direction of development as a whole, and for this purpose we must make use of valuations which open up all the ultimate questions of meaning and validity. It will moreover appear, I hope, in the sequel, that the conception of development in its turn throws no small light on these ultimate questions. The advantage to the two branches of the enquiry is mutual, and if we could arrive at no satisfactory conception of the trend of development without a theory of the rational and the good, it will be found equally that our conceptions, and equally our misconceptions, of the rational and the good are intimately connected with the idea of development.

To put the matter very simply, the object of our historical enquiry is to measure the growth of mind from the lowest to the highest phase of development. But how are we to know which is highest? The term itself implies a valuation, and unless we have a reasoned standard of value we have no scientific means of determining the terminus ad quem of our narrative. We certainly cannot take our own civilisation as the highest product of the social mind without any dubiety or any reasons given. It does not, to say the least, stand so high in its achievement above some earlier civilisations which arose and flourished and passed away. Human development, it is well to recognise from the outset, does not proceed continuously in a straight line. If we make the civilisation of our own day the 
terminal point of our narrative we have still to ask whether this point is the 'highest' yet reached or whether it marks a decline from some earlier stage, and this is a question which can only be determined by the aid of a standard upon which the 'higher' and the 'lower' are clearly marked. If in the end we come to the conclusion-as for reasons which will be given, I believe we may-that our own civilisation does upon the whole represent a certain net advance on the previous efforts of humanity, this conclusion must be based upon a clear-sighted comparison of the historical facts with an agreed standard of values.

7. Lastly, the most interesting class of questions concerning development cannot be answered by history alone. A just conception of the trend of development is most valuable to us in as far as it concerns the future. But though history may suffice to show us the orbit upon which the evolution of mind has moved we cannot project the curve into the future by the aid of history alone. We shall have to investigate the permanent conditions of mental growth, and when this problem is taken in its broadest aspects it will be found to compel an examination of the whole position of Mind in the system of Reality. This investigation must decide first whether Mind is, as suggested by the mechanical theory, a mere 'epiphenomenon' or a substantive factor in evolution, and secondly, if it is a factor at all, what position it holds and what function it performs. This will necessitate an analysis of the causal process, which will again involve an appeal to first principles. This analysis will occupy the second part of the volume, and the attempt will there be made to show that by its means we arrive at a conception of Mind and its function in Reality which is in close harmony with our historical results and which accordingly serves to corroborate and extend the interpretation which they suggest.

The scope and method of the book then may now be briefly defined. Its object is to determine the nature of Mind and its position or function in the system of Reality and its method is first to trace the historical development of Mind from its earliest ascertainable conditions to its latest phases, secondly to value the achievement of these 
phases by a philosophical analysis, and thirdly, to apply the results of analysis to the elucidation of the efficacy and scope of Mind as a cause. It will be argued finally that the historical review and the philosophical analysis converge upon the same result, in the sense that the process of evolution when viewed in its completeness serves as a concrete verification of the general theory of Mind which analysis suggests, while conversely the theory serves to interpret and explain the course of evolution. If this is so, we shall have some ground for the belief that our metaphysical conception of Mind is not a piece of abstract reasoning that stands in no contact with living fact, but serves as the explanation of a vast historic movement. At the same time we shall have reason to think that this movement which we trace through the whole sweep of terrestrial evolution is no secondary and isolated result of a unique collection of circumstances, but is of the essence of the world process. Our empirical account will in fact yield us a picture of Mind neither as the Lord of all, nor as the casual bye-product of the clash of forces, but as an impulse towards organic harmony working under limiting conditions which it gradually subdues, and in such an impulse on a still vaster scale we shall find in the end the most reasonable interpretation of the vital process of the cosmic order. 


\section{CHAPTER II}

\section{THE STRUCTURE OF MIND}

\section{(I) Consciousness and its content.}

What we know of mind together with what we know of the world in general is derived in the last resort from the sum of that which comes before our consciousness. We experience, we think, we desire, we purpose. In all these cases we are or may be conscious, and in all there is in ordinary phrase some object with which we are concerned. The phrase is not free from difficulties. It carries associations which are ambiguous and even contradictory. Thus on the one hand it appears as a relative term, implying a subject on which it depends. If there is an object of sight, of hearing, of thought or purpose, there must, it may be said, be a subject which sees, hears, thinks or purposes it. On the other hand, by a contrary turn of association, the object, it may be held, is just that which is independent of any subjective element and in using it we may seem to be assuming a theory of external reality. But as here employed the term is to convey neither meaning. The object is something which we see, hear, think of or purpose, but by calling it an object we do not imply that its existence depends on one of these acts. Nor again do we imply the contrary. All that we do maintain is that the act of consciousness has an object. We feel, see, think or purpose something. The former terms are grouped together as acts or modes of consciousness. The 'something' is the object of consciousness. The statement however implies that we can know that we think, feel, etc., which is as much as to say that the mode of consciousness, the act of enter- 
taining or contemplating an object may itself become the object of a conscious act. It is from this secondary or reflective consciousness, or if the phrase be preferred, it is from these elements in the objects of consciousness that the beginnings of our conception of mind, self, personality, appear on a first view to be derived.

This view however may be challenged from more sides than one. In the first place, it may be urged on etymological grounds that the term consciousness is inappropriate as an expression for any direct operation of knowing, feeling or willing. To see or hear is one thing, it may be said. To be conscious that I see or hear is another. It is something that implies two co-ordinate or concomitant operations, seeing and being aware of seeing, and it is just this doubleness that the form of the word conscious conveys. To this it may be sufficient to say in reply that the use of a term is to be settled by convenience rather than by etymology. It is indeed necessary to distinguish the grades of complexity in different contents, and it is true that there is a valid distinction between seeing and knowing that we see. But underlying this specific distinction there is a more fundamental and generic identity. There is in the simple as in the more complex case something that is aware, something that has an object before it in one way or another. We need a name for this something and the name consciousness serves our turn. Consciousness is that which has before it, has present to it, is aware of some object or other. The term serves as a grammatical subject in any one of those sentences. Neither the subject, nor the verb, nor the predicate appear to be capable of further definition in the sense of resolution into simpler or more general elements. They are on the contrary general conceptions to be defined $(a)$ by enumerating the specific types which fall within them, and $(b)$ by distinction from allied conceptions with which they may be confused.

But here a more serious criticism emerges. We have treated 'consciousness' as a subject in a sentence, and this is as much as to imply that there is a distinction and also a relation between consciousness and its object. We have come to know this relation, our preliminary account 
suggests, by a more reflective act of consciousness. What is subject at one moment is object at another. I see Halley's comet, and so far the comet is my object. But I also report the seeing, and here the conscious state qua conscious has itself become the object of my thought. But in attending to the process of seeing, must I not lose hold of the object seen? And yet if I do so, is not the process of seeing at once vitiated-destroyed therefore in the act of apprehending it? This is, it must be admitted, a real difficulty in introspective psychology and has even led some to deny the validity of introspective methods altogether. It is not however necessary for our purpose to probe all the difficulties that surround the question. Our direct consciousness of mental process is sufficiently clear to found a general conception of conscious life and activity, to enable us to recognise the leading species of this activity, and to infer its operation from results in cases where it is not directly given. This will be all that our account will be found to assume.

\section{(2) Mind and Consciousness.}

If this assumption is justified there is no initial difficulty in conceiving the 'operations' of consciousness or its varying relations to its object as the elements out of which our conceptions of Mind and Self are empirically constructed. It is with the former conception that we are especially concerned and we have to examine its logical foundation. Consciousness, as appears from our previous account, is a name for a state, an act or a condition, in short for something temporary. We seek for something more permanent to which we can refer it, for the same reasons which make us impute colour, sound, length or weight to material substances. Into these reasons we need not enter here. It will suffice us for the moment that we give the name of Mind to the permanent unity of which we conceive any given act of consciousness to be the temporary condition, act or state. But it may be asked why, granting the desirability of something permanent as the vehicle of consciousness, should we look beyond the body, a permanent object which we are already forced to construct by 
the evidence of a mass of common experience. Is Mind another entity; is it a substance like the body, and if so how are the two related?

If we are to deal, however summarily, with these questions, we must first review the body of experience which underlies the conception of Mind as a distinctive unity. This experience, to state the facts very generally, yields two data. The first is that the subjective factor, which we have insisted on as an element in consciousness, is for each one of us a permanent element. It is always there when consciousness is there. It is the same ' $I$ ' that feels hungry, or cold, reads a book, climbs a hill. It is the same ' $I$ ' which memory gives me in the remote past and anticipation projects into the future. What does 'the same' mean here? Not certainly that I am unchanged, but that I can view my conscious life as a whole in which there is a certain thread continuing throughout, and retaining amid change a certain element of persistent character. This continuity in consciousness is not indeed the whole, but it is the core of the 'I.' But consciousness itself is broken, e.g. by sleep, and the sense of an unbroken continuity which unites me to my past would be illusory if my existence depended on consciousness alone. This brings us at once to our second datum. This is that the facts of consciousness reveal upon examination the working: of causes strictly continuous with those that appear within the field of consciousness itself, but yet extending outside that field. There appears in short to be something that operates unconsciously, but yet in a manner closely comparable and even in essence identical with many of the operations familiar to us as operations of consciousness. Moreover by these operations, proceeding as it were in the background, the attitude of consciousness is in a large measure determined. Conscious and unconscious operations then may be legitimately grouped together, and without prejudgment as to their ultimate nature the sum of them may be called Mind. Mind then appears as that which has consciousness in its foreground while in the background it is the theatre of energies, of interactions, of stresses and strains, the play of which goes to determine the character 
of the scene by which the said foreground is filled. To understand this relation, not in its metaphysical essence, but in its empirical detail, is highly important for our purpose.

We may approach the question by a simple and familiar analysis of the ordinary content of every-day consciousness. As I write these words my interest is concentrated directly upon the idea that I am seeking to express. This forms the 'Blickpunkt,' the 'centre of vision' in the field of my consciousness over a considerable space of time. From this centre many other elements are from moment to moment determined. The central idea expresses itself in words, of which the most important are matter of conscious choice occupying therefore for the moment the centre of attention. The lesser words, the 'ifs' and 'ands,' come more automatically under the influence of the meaning which the sentence has to express, and the actual writing is of course in the main a more or less mechanical process. This part of the proceeding however is psychologically not the least interesting. Consciousness, though barely occupied with it, retains control over it, sufficient, for example, as a rule to notice and correct a slip, though in fact success in this respect will be in inverse proportion to concentration on the main idea. The detail of the writing then is half within and half without the control of consciousness. It occupies a marginal position. Yet beyond it there are still further and dimmer sense-elements; the objects on the table coming within the sphere of vision, the sense of sunlight and chirrup of birds out of doors, the permanent background of organic feeling. Lastly, the thinking process may be vaguely disturbed by a sound which presently reveals itself, as the striking of a clock, and in this case there may be the paradoxical effect that the strokes which I definitely hear are the last two or three, but that when I notice them I am at the same time aware that there have been several previous strokes. I am in fact aware of having heard these strokes though I was not at the moment aware of hearing them.

So far this brief and familiar analysis shows that in consciousness there is every gradation in the fulness and 
distinctness of presentation from the maximum of clearness to a zero, and also to something below this zero. The field of consciousness appears not like a material object with clear-cut outlines, but more like the halo of light which a lamp projects into the darkness. There is a gradation from the focus of the rays to their extreme verge, and the outline of light is not clearly marked. Light fades away into darkness. But that is not all, and when we pursue the matter further the image of the lamp requires modification. For not only is there an oscillation between the light and the dark which we might compare to the effect of a swinging of the lamp, but what goes on in the dark area affects the lighted area just as if it had passed there. I do not refer merely to the marginal sensations like the striking of the clock. I refer to the causes operating normally on the definite elements of content within the field of perfectly clear consciousness. Thus in my example I spoke of the words suggested by the central idea. How does the idea come to suggest these words? For the most part not through any conscious process of which I could render account, but by the reaction of the present purpose on my antecedent knowledge of my mother tongue. A host of experiences relative to the use and meaning of words, experiences long forgotten and perished beyond recall in their individual character are the influences which have furnished me with whatever expressions I have at command. But observe that this process of suggestion may itself at any moment become conscious. Thus when in the previous paragraph I wrote the word 'Blickpunkt' a conscious recollection of a well-known passage in Wundt's Physiological Psychology operated in my mind, and there even arose in it faint images of the room in which twenty years ago I first read that work. It might quite easily have happened that I retained the word and forgot Wundt, but the fundamentals of the process would have been just the same. Similarly if a question occurs as to the suitability of any word, the processes which suggest it, the relations of meaning, the grammatical or etymological connections are called up into consciousness. They are, as we always say, rendered explicit. They are 'there' already by implication, and 
need only a movement, a re-direction of attention to be brought forward as distinct objects. In this respect their position closely resembles that of the sounds of the clock which I am aware of as soon as something calls my attention to them, but which otherwise may pass unnoticed. They are 'there,' but not known to be there. 'That is to say, they are in the Mind though not in consciousness, and that again, to keep to facts which we can verify, means that they belong to a mass of operative elements continuous with consciousness, capable of figuring in consciousness, influencing the contents of consciousness, but not necessarily at any given time distinct elements in the content of consciousness. To use once more the figure of the lamp, consciousness is at any moment the area indefinite in its. boundaries in which the light falls. Mind is the whole area which the lamp, as it turns this way and that, is capable of illuminating.

We may profitably carry the figure a little further. Let us suppose that in the sphere around the lamp many things are going on which intimately affect one another. It is a field of interacting forces, which are only to be thoroughly understood when understood as a whole. Let us suppose that the lamp is swinging in all directions so as to illuminate the whole area in turn. An observer would then have the entire data before him for understanding the processes in question. He would obtain them piecemeal, but he would be able to put his results together, and there would be no source of information from which he would be entirely cut off. Suppose, on the other hand, that the lamp was so pivoted that it would only swing in one plane, or perhaps that it was even limited to a section of that plane. The observer would then be in very different case. He could only obtain a fragmentary knowledge. If anything were so arranged as to occur regularly in that plane he could forecast its behaviour, but without adequate knowledge of the underlying forces. Suppose, finally, that after being limited to a segment, the lamp were set free to sweep the whole circumference, and after being limited to a plane were set free to sweep the whole sphere. The spectator would then be aware of a complete change in the point of view, carrying 
him below the surface to the real causes of the events transacted before his eyes. It is by a change involving a reorientation of this kind that the mind that has been limited to the surface of experience comes to apprehend the deeper causes of things. These causes may be external, or they may be forces operating within the depth of the mind itself. In either case they require an enlargement of horizon and change of direction in order to be brought within the direct purview of consciousness.

The matter of re-orientation, with the consequent bringing of the unconscious into consciousness, is one of which we shall have a good deal more to say at a later stage. But let us here put together the two main data which experience yields for our conception of Mind. We have first the presence of the subject in consciousness. The only positive objection to conceiving the subject as the permanent unity which we require for the changing states of consciousness was that its permanence is broken along with that of consciousness itself. This objection is removed by our second datum, which yields the conception of a sum of partly conscious or quite unconscious process surrounding and determining consciousness. This sum of process we can take as permanent, and as having for its distinctive character that it either is or at any time under appropriate stimulus can become the subject in an act of consciousness. So conceiving it we call it Mind.

\section{(3) Mind and Body.}

But it may be said all those unconscious influences that surround consciousness, which constitute what we have called our 'second datum,' are so many witnesses to the sole sufficiency of Body as the true subject, the permanent unity of which consciousness is a state. The brain is a physical structure interacting with other physical objects. One of its peculiarities is that when it acts in a particular way, when, for example, certain of its areas are traversed by waves of excitement, there arise the phenomena of feelings, ideas, and all that we know as consciousness and its content. Other brain reactions are in their main physical characteristics similar to these, but are not accompanied by conscious- 
ness. It is perfectly intelligible that they should affect those special processes with which consciousness is concerned, for all parts of the brain stand in intimate physiological relation to one another, and thus it happens that the basis of much that goes on in consciousness is to be found in molecular interactions not accompanied by consciousness. Mind is really brain and nothing more. No other permanent subject is either directly experienced or implied by experience.

Now the body is no doubt a continuous unity with whose functions conscious activity stands in close relation. But it is not a 'permanent subject,' because it is not strictly a 'subject' at all. To identify mind and body in the sense of resolving one into the other is simply to confuse distinct categories. Body, as known to us, is that which is measurable and ponderable, that which has mass, which moves and is moved, is visible, tangible and so forth. Mind is that which feels, sees, hears, judges, expects, infers. To say that mind is body is as much a confusion as to say that a weight is an inference or that an acceleration is a wish. Very slight consideration shows that if mind and body are to be identified in any intelligible sense the meaning must be that in any individual they form one permanent reality whose attributes include on the one side the phenomena which we group as physical, on the other those which we group as mental. Such a reality would be a psychophysical whole, which we may call the Self. This conception may pass as a prima facie account, and it serves to put the question of substance in the right form. For at bottom what we have to ask is whether the mental phenomena depend on the bodily, or the bodily on the mental, or whether there is some interaction between the two. That is to say, to understand the relation of mind and body we ought to know whether the totality of the processes going on within the self is to be understood in mechanical terms as a series of actions and reactions of masses in motion, or in terms of mind as a series of efforts determined by purposes. Now the only sound method of approaching this question is to consider the self as a psycho-physical whole, and to enquire how it acts. In point of fact this enquiry 
enables us to arrange the actions of the developed human self in a series, the first terms of which are prima facie mechanical in character, i.e. are explicable in terms of the interaction of masses, while the last are prima facie mental, i.e. are explicable only in terms of purpose. Whether this prima facie view can maintain itself to the end or must yield on analysis to the theory that the most developed purpose is the result of a peculiarly complex mechanism is a question which must be taken up again when the exposition of the series is complete. In the meantime we shall deal with mental activity and mental determination as we find them, and make it our business to describe the forms which they assume and the part that they play. By this means we shall trace the development of the mind-function within the self, though we shall leave open the question whether the whole of this development is or is not to be interpreted ultimately in mechanical terms.

The question thus left open is one of the ultimate nature of causation in the psycho-physical process. We shall nowhere have to challenge the view that a mental state or process implies a bodily state or process as concomitant. We shall only have to ask in the end whether as between these concomitants we are always bound to look to the bodily side for the real explanation of the process. We need not doubt that when I see a ball coming towards me and put up my hand to catch it there is a physiologically continuous process ${ }^{1}$ from the excitement of the retinal cells to the series of muscular adjustments which results in the catch accompanying the mental acts of perception and conation. We need not doubt that when I read a book the train of reflections set up and issuing ultimately perhaps in written and spoken words of my own implies a long series of physical adjustments in cerebral cells. Only, having this circumstance before us, we must insist equally on both

${ }^{1}$ Some psychologists (as Mr. MacDougall, Body and Mind, pp. 288, etc., cf. Sherrington, The Integrative Action of the Nervous System, p. 384 ff.) throw doubt on the spatial continuity of the processes involved in some mental operations. I am not qualified to form any judgment on this question. All that is meant in the text is that for the purposes of the present argument no discontinuity need be assumed. 
sides of the relation, and by so doing we may justly extend our conception of the psychical life on the one side just as we extend the physical life on the other.

The physical processes associated with consciousness are changes of the nature of which very little is known in the mass of interconnected nerve cells and fibres constituting the hemispheres of the brain. There is no known difference of fundamental quality between these processes and those which go forward in lower nerve centres, and which are not normally attended by consciousness, while there are many acts which are performed sometimes consciously, at other times, if attention is otherwise occupied, unconsciously. It is a fair inference that on the physical side there is no gulf between the processes attended by consciousness and those not so attended. But we have already seen that on the mental side there is true continuity of character, the conscious shading off from the clear light through every gradation of dimness to the utter dark, while that which was dark may under new conditions enter into the light. The inference is that organic processes which do not involve clear consciousness may yet include a psychical element, or, more accurately, that the psychical concomitant of neural process may be regarded as varying from a maximum to a zero point, which is perhaps reached in the cases which we shall find in which a reaction has become once for all stereotyped. Thus we may take the psycho-physical whole as a continuous unity, the differences within which are either differences of degree or at most differences of species within a genus. Our business then is to consider the general character of the behaviour of this unity, and then to set out the specific differences of its functions in such a way as to exhibit the various phases of the psychical factor from its lowest to its highest forms. 


\section{CHAPTER III}

THE GENERAL FUNCTION OF MIND AND BRAIN

(I) The portion of the body with which mind is associated is the central nervous system. Now the function of the nerve tissue in general is to secure the correlation of different parts of the body in the work of adaptation to its needs and to those of the race. The temperature rises, and a nervous mechanism responds by expanding the smaller arteries, distributing the blood over the surface, and increasing the activity of the sweat glands. By these means the blood is kept to an even temperature. A blast of cold air or douche of cold water produces the reverse effects. By running or making any muscular effort we denude the blood of oxygen, and the result is to stimulate the respiratory centre to a greater activity, which causes us by panting to absorb more oxygen. As we run we catch our foot against a stone, and the other foot comes up more quickly to preserve the balance, or the hands fly out to protect the face in falling. These adaptations are performed for the most part without the aid of consciousness, which is not quick enough for them, by means of arrangements of sense organs, nerve fibres, nerve centres and muscles, which can in many cases be traced in considerable detail. The nerve fibre is essentially a conductor of excitements. It leads, say, from a cell of the retina to a cell of the mid-brain, and from this cell another fibre will proceed, conducting the excitement to a cell of the occipital lobe. Arrived at the cortex or grey matter of the occipital lobe, the excitement is propagated in a cell of ' pyramidal' form, possessing very complex branching processes, which intertwine with the pro- 
cesses of neighbouring cells. ${ }^{1}$ We explore the brain fruitlessly for any one centre to which all things are brought and from which all start anew. What we find is myriad on myriad of ramifications so arranged that any disturbance may propagate itself through the whole area and awake response from any cell whose stored-up energies are sensitive to its stimulus. Undoubtedly there must be precise conditions determining which cells will respond to a given stimulus and in what way. But as to this we know only the broad empirical fact that the response is in general one that is suitable or at least relevant to the situation, and that the effectiveness of the response depends on the maintenance of functional continuity between the nerve fibres which constitute the paths of communication. The central system appears in short as an exceedingly complex system of intercommunication, by means of which, to put the matter in very general terms, any element in our experience may be brought into relation with the whole mass of our stored-up energies in such a way as to facilitate orderly and consecutive action.

The matter may be made a little clearer by reverting to the scheme of reflex action and its inhibition as ordinarily described. If I withdraw my hand sharply from contact with a hot object the process is explained physically as a reflex. The contact with the skin is held to send a nerve excitement to a ' sensory' cell, which again propagates it to ' motor' cells, which in turn give rise to impulses descending the motor nerves and resulting in muscular contractions of the hand and arm. But if it is a point of honour or of safety not to flinch but to hold on, what happens physically, it is conceived, is that the excitement in the sensory cell passes along other fibres besides those which lead to the motor area ; that it awakens in turn other cells in different portions of the brain, and that these by one or other of the

${ }^{1}$ The available evidence goes to show that the processes of different nerve-elements are not, in vertebrates, in actual contact. The point of interconnection between them is called a synapse, and it is probable that to pass the synapse the excitement has to overcome a certain resistance, the strength of which, as compared with the resistance at other synapses by which the excitement might find outlet, is probably of high importance in determining its path. 
infinitely numerous connecting fibres quell the tendency of the motor centres to discharge. ${ }^{1}$ The reflex impulse is thus inhibited, and I hold on in spite of pain. Physically the interpretation is of the same order all the way through. The difference is that in the reflex the system of intercommunication is simple, providing only for the undisturbed flow of excitement in one direction, while in the case of inhibition the system has developed, and the wave of excitement sets in motion energies in other parts of the brain-mass which cancel its original movement. The effect of this development is to bring the stimulus of the moment into relation with other and more remote vital functions, to increase the extent of correlation between different parts, or incidents, of the entire activity of the organism. And in effect it will be observed-no matter as yet by what method-the correlation transcends the present. The act is performed or restrained in virtue of effects which will accrue in the future, perhaps the remote future. At the same time the influences operating to promote or restrain it may derive from the past, perhaps the remote past. Expressing the same thing in terms of mind, what we should say of course is that present pain is discounted for the sake of some wider, deeper or remoter end, my safety or my credit. Whatever the nature of the end, the obvious point is that the experience of the moment, instead of being left isolated, is connected with other experiences contemporaneous, past and future, and perhaps with my life as a whole. Now to achieve such interconnection and thereby to order behaviour is, we may say, the generic function of Mind regarded as a factor in life, and we can thus easily see that the functions of Mind and of the nervous system are generically the same. Specifically we shall find that there are forms of correlation in which the psychical factor is unimportant or absent, while

1 The structure of the nervous system is specially adapted to the inhibition and equally to the co-operation of reflexes by the fact that many paths of conduction unite through synapses in common paths. If two or more excitements end in the "final common path" leading to the same muscle, they naturally cancel one another if opposed and strengthen one another if allied (see Sherrington, op. cit. esp. Lect. IV.). 
there are others which do not proceed without it, and are (at lowest) correlated with specific stages of its development.

(2) The measure of this development is the area which the conscious life controls. Not only is conscious activity the only ground which we have at the outset for imputing mental activity, but further we may regard consciousness as being the organ by which the mind effects correlation. Indeed we may go further and say that, whatever the ultimate truth as to causation, at the level of development which it has reached in human beings, the psycho-physical whole, which we have called the self, does not ordinarily effect new correlation without some consciousness of what it is doing. I say not ordinarily. In the body functions best performed and normally performed by one organ may be indirectly and cumbrously brought about by means of others. The skin performs in a rougher way some of the functions which are specifically those of the lung and the kidney, and the organism that has lost the services of any organ makes shift to do without it by bringing up reserves of energy. Yet there is no doubt in this case as to the nature and function of the specific organ. Similarly we shall see in the case of mind that correlation is slowly, indirectly and inefficiently performed outside or partly outside of consciousness, while it becomes swift, direct and efficient in proportion as it enters the conscious area. Thus if a painful experience attends a response of a certain kind at a low grade of consciousness, a fitful, uncertain and gradual modification of the response will ensue. At a higher grade the relation of the response to its consequence is definitely grasped, and there is an immediate and decisive alteration of behavicur. It is in this sense that consciousness is the organ of correlation. Perhaps the simplest evidence of its specific function is to be seen in methods of correlation which consciousness itself establishes, but which, when rendered thoroughly familiar, need no further consciousness for their execution. This is the familiar experience of our daily habits. We can walk, run, ride a bicycle and so forth without thinking about what we are doing. We all know how that which is learnt with 
the expenditure of laborious and painfully conscious effort passes rapidly as it becomes perfect to the margin of the conscious area or altogether beyond its limits. But conversely in these very exercises, as soon as some conjunction occurs requiring new and perhaps unique adaptation, conscious attention comes again into play. It is through the elements that come into consciousness as such that we principally establish new correlations, and we may take as the external sign of the birth of consciousness the appearance of a permanent power of making new combinations, while the measure of the growth of consciousness, and therewith of mind, is in the extent and perfection of the combinations which we can form. In particular we shall find that the extent to which the factors influencing consciousness are themselves brought within the content of consciousness is of special importance in estimating the growth of mind.

We conceive then of the psycho-physical unity, which is the self, as the seat of mental and of physical phenomena. Under either aspect we can regard it as a unity which subsists by processes of adjustment involving the correlation of different experiences and energies. In the nervous system we see the physical basis of such correlation. In the mental life we see it clearly at work, and proceeding at its best through the medium of consciousness. Our business will be to classify the different forms of adaptive correlation and to distinguish the sphere of consciousness in each. We shall thus arrive at a conception of the development and the sphere of mind which will be true, so far as it goes, whatever interpretation we may ultimately put on the causation of mental phenomena. For this purpose we must first review the general conditions under which the whole psycho-physical unity works.

\section{(3) The Psycho-Physical Structure in its Development.}

Let us take any commonplace deliverance of consciousness and consider the general conditions on which it rests. As I write I hear a lark singing outside. This perception is not the effect of the lark's song alone, nor of the physical waves of air that beat upon the drum of the ear, nor of the vibration of the membrane of Corti, nor of the 
wave of excitement that traverses the auditory nerves. It is a reaction of something, call it as you will, mind or brain, but certainly of a preformed structure. To the fashioning of that structure there have gone in the first place certain factors of heredity, in the second certain factors of experience. Of these last the most obvious is that I have heard similar sounds before, and have connected them with a bird, and have been told in childhood that that bird is called a lark. If I had to justify my original judgment I should have presumably to advert to experiences of that kind. My perceptive judgment would appear as a kind of inference in which previous experiences figured as an inductive premise, and it is very easy here to fall into the confusion of supposing some such inference actually to take place when I merely give a thing a name. It is tempting to break up the process into elements - as (a) a certain sound, $(b)$ the subsumption of this sound under a general conception of lark's song, and (c) a concluding, inferential judgment ' that is a lark.' In actual consciousness of course nothing of the sort takes place. What has actually happened is that past experiences have so prepared the mental structure that it reacts to a given physical stimulus with the judgment ' that is a lark.' 'The chain of causation is parallel to that of the analysed inference. The same elements are there, and the effect is the same, but they are never, except as now by a writer seeking an illustration, analysed out and then put together in an articulate whole. This relation is general. On all sides experience leaves results on the mind-structure which function as inferences, but are not inferences. Very often we cannot on being challenged discover through memory the experiences which have caused the modification. An object is charged with emotional suggestions, a scent or a colour-pattern stirs our liking or disliking, and we can find in the recesses of memory no experience to account for it. The results of old experiences are for us woyen into the texture of the object. More accurately they have come to qualify our perceptive reaction to a stimulus. The content of this perception therefore, and in particular the feeling-tone which qualifies it, may be said to stand for and reflect in the mind the nature of the experi- 
ence, perhaps of a whole mass of experiences, in which similar perceptions have in our previous history been concerned.

(4) But it is not only our own history which forms our mental structure. The experience on which a suggestion rests may be irrecoverable by memory, for the simple reason that it never occurred within the range of memory. The mind, as part of the whole psycho-physical structure, grows up under the influence of heredity as a whole, and in its several parts it arises, survives and is modified from generation to generation in accordance with vital needs. The main need which the mind functions subserve is that of directing response to the environment, and the direction must in the main be that which tends to the preservation of race. Under these influences arises a mind-structure endowed with definite tendencies of reaction, quick, for instance, to respond with perception to certain external movements which threaten the safety of the organism, and not only with perception, but with appropriate motion and appropriate feeling. Here again we must in any individual case be on our guard against the old fallacy. When we see a fish dart away in response to a sudden movement of our own we must not hastily impute to the fish a series of distinct operations-such as the perception of a moving object, a fear of attack, and resolution to fly. For all we know the fish may be capable neither of perception, emotion or resolve. What we see is the responsive motion, which would be logically justified by the fear of danger, which fear again might be logically justified by an experience of men and of their unkind dealings with fish. If the fish is capable of mental processes, and if these mental processes correspond, as they may, to certain of the lower processes of our own mind, we may put it that what actually passes in the supposed case is a process which contains all the elements enumerated in germ, but none of them in maturity or distinctness.

For among ourselves the primal basis of our reactions is not reflective. It is not even due to experience. It is a part of that original equipment which we call hereditary, ${ }^{1}$

${ }^{1}$ We know not where to look for the source of any element in our original equipment except in the physical antecedents of our birth. So 
or in more familiar phrase it is instinctive. This hereditary element is not to be confined to certain specific elements in our mental life, to certain 'forms of thought,' certain principles of will, certain types of emotion. No doubt there are points at which its influence is more distinct, less overlaid by the effects of social tradition and personal experience. But rightly understood it permeates the entire life of mind. In a sense its operation is most decisive in the very department which is singled out as the especial preserve of personal experience, the department of 'pure' sensation. So far as a sensation is 'pure,' that is to say is unmodified by elements of thought or by the unconscious operation of previous experience, it represents the naked reaction of the hereditary structure of mind on the given stimulus. The poppies are red and the oak leaves green to us because our organism is so constructed as to react to the physical stimulus of vibrations of different wavelengths with those two sensations. That the one object is a red poppy and the other an oak leaf are judgments in which something more than pure sensation is involved. That they are red objects is a judgment in which something more is involved. That the names red and green apply to them are judgments in which something more is involved. But in the quality of the sensation 'red,' 'green,' we come as near as we ever can to pure sensation, and therewith we come to that which depends on the original hereditary endowment of the mind-structure. This element will be found accordingly to pervade our judgments of external things. It is even more obviously present in our feelings and our impulses. It is operative in our judgments and inferences. It is the original foundation of our temperament and character. But all along, until we reach the highest stages of reflection, it is in its operation unconscious. That is to say it determines our

we call the whole of it hereditary. But (a) there may be other sources of which at present we know nothing, and $(b)$ the use of the term 'hereditary' does not imply any specific measure of likeness to our parents. From generation to generation there is variation as well as resemblance, and exactly how much there may be of either is a question for the science of genetics to determine. 
mode of reaction, decides the way in which we take things without our knowledge of its operation. Though a man may in part know himself in the sense of being aware of certain idiosyncrasies of temper and on his guard against them, it is only science dealing with nerve and brain, heredity, education, and the reaction of body and mind that can render in fully explicit terms the true nature and limits of the hereditary factor.

(5) Enough however will have been said to show that the ground layer of mind is a property of the hereditary structure. Upon this foundation experience works, but the result at any moment is not to be severed by any mechanical process into effects of experience and effects of heredity. The result is the product of a continuous process of interaction, and will accordingly be a function of both the contributory factors. There is, however, one element common to the two. The hereditary element is itself shaped indirectly by the experience of the ancestral stock. The stock has had to live and act within a world of experience which is on the whole the same world, and it has had to adapt itself to that world or perish. Hence, in the basis of the individual constitution lie tendencies, modes of feeling, promptings of action making in the main for sanity, making at least for the race preserving as against the race destructive line of conduct. These tendencies may be so precise and complete as to determine action without the need of any individual experience to perfect them. They then form the basis of inherited reflexes or instincts. Or they may be vaguer and more general, and may figure accordingly as promptings, tendencies, characteristics, or mere potentialities which the experience of life serves to define and complete. The first and more developed form plays the more important part in animal life where the scope of consciousness is smaller. As the sphere of conscious correlation grows so there is less room for fully developed specifically determined modes of reaction, and the function of the pre-existing structure is rather to form a basis for correlations which are constantly effected anew by fresh mental efforts. Hence, though the hereditary equipment of man is not poorer but richer than that 
of the animal, it is an equipment which is less complete in itself and leaves more scope for the exercise of initiative. Man has many instinctive tendencies, but few instincts complete in themselves.

At any rate, what we have to emphasise here as of the first importance to the student of consciousness in its development is the existence of a permanent background, the work of the massive inarticulate action of ancestral experience as modified by the half-articulate action of personal experience and the social atmosphere. These forces together form that permanent basis of our thought, action and feeling which Lady Welby has called the mother-sense. This is something less specific than instinct, judgment, inference, or will. It is not so much the immediate determinant of specific acts, though it does lead to specific acts - to precisely those acts which we perform with confidence, though admittedly without being able to give our reasons. It barely enters into consciousness as a distinct force, though it is often what lies close upon the verge of the luminous area when we claim an 'intuitive' certainty of something, when a situation impresses us as hopeful or threatening, or a personality as attractive or repulsive without tangible ground. In another aspect it is itself closely allied to the foundations of that same 'personality' which impresses, or fails to impress, others, in apparent defiance of the qualities that men praise or blame, admire or condemn. It is as the enveloping atmosphere of the conscious life, or to take a different metaphor it is a mothersubstance, a matrix out of which clear-cut contents of consciousness, explicit judgments, purposes, stated reasons can be taken. But what is to be remarked about the contents so taken is that in the process of cutting they are often more or less mutilated. If we seek, for example, to analyse the qualities of someone whom we admire, we succeed perhaps in fixing certain points. We can formulate the basis of our judgment to a certain extent, but very often we are quite conscious that this is not the whole account of the matter, and when we are not so conscious we no less often mistake ourselves, and impute reasons which are inadequate and unreal. The distinct quality assigned 
as the basis of a feeling or a judgment is, in short, the result of an effort of analysis, and analysis is a partial attempt to crystallise what is fluid, or to distinguish and map out what is originally present in consciousness as a whole. Now this process of distinction and systematisation is the basis of all the higher developments of mind. But it is at the same time to be understood that it arises and performs its functions within the sphere of the 'mothersense,' and its business is to replace the unreflective deliverance of the mother-sense by an articulate system of thought. In one sense the defined idea is from the first an advance upon the obscurer reactions of the mother-sense. It is more articulate, more rational. It is a necessary step towards the full consciousness of developed mentality. But in its use there lurks from the first a source of fallacy - the danger of being guided by a partial and incomplete analysis, a danger which may lead to practical mistakes from which the simple confidence in the untroubled mothersense might be free. What we can satisfactorily formulate being seldom more than a part of the reasons really influencing us may omit something that is essential, and so we get all the errors of the 'abstract' type of mind. Of these we shall have something to say at a later stage. In the study of mental evolution they may best be guarded against by bearing constantly in mind that explicit consciousness does not suddenly arise in full definiteness out of the void, but emerges within the sphere of the mothersense and remains until the highest stage of its growth under the influence of forces which it comprehends imperfectly or not at all.

Our argument then has led us to conceive Mind, whether in man or brute, as part of a psycho-physical structure which grows under the conditions of heredity and is modified in each individual by experience. This structure reacts in accordance with the laws of its constitution to that part of the environment with which it comes in contact, in such a way generally as to adapt the actions of the organism to the needs of race-maintenance. The method of adaptation in which Mind is specially concerned is the correlation of one experience or one act with others, and we may 


\section{DEVELOPMENT AND PURPOSE CHAP. III}

regard all such correlation as partaking of psychical character. Its special organ is consciousness without which new correlations are only effected indirectly and cumbrously. The development of Mind can accordingly be measured by the nature of the correlations effected and the conditions under which they are effected, and in comparing these we shall find every gradation from the case where the activity of consciousness is at zero, to that in which it is the effective determinant of the entire function. 


\section{CHAPTER IV}

MENTAL DEVELOPMENT IN THE INDIVIDUAL

I. IN mental as in physical evolution, the emergence of new factors does not involve the total disappearance of the old. These are merely overlaid and in varying degree modified by the later development. Just as man remains an animal, so the most reflective consciousness coexists with the most irrational impulse and the life of the most perfect and complete human being has its roots in methods of action and reaction which it shares not only with the life of the savage or of the dog, but with that of the rhizopod or the plant. Thus we get in the developed man a rough epitome of the history of the race, we find in him modes of action which represent all the stages which the race has passed through. The correspondence is not indeed accurate, for the presence of new factors modifies the operation of those which are older, but (as in embryology) it is sufficiently near to enable us to form a rough outline of the evolutionary process, an outline which we can verify and correct by comparison with the actual behaviour of animals at different grades of development.

We may therefore suitably approach our task by distinguishing the elements discoverable in the activity of the developed man, and considering their analogues in the animal world. In doing so, since we conceive the organism as a psycho-physical unity, we shall take physical reactions into account along with the deliverances of consciousness, using, in any case, the evidence most readily accessible and most easily verifiable.

Having taken correlation to be the typical function of 
mind in relation to the life of the organism, we shall found our classification of reactions on the nature and conditions of the correlations involved. Now, generically correlation is a term applied to the parts of a whole when they are so arranged that their joint operation yields a result tending to the maintenance of the whole or of some function, character, or activity of the whole. ${ }^{1}$ The source and nature of the arrangement may be very various. In a machine it is one thing, in an organism another, in the colour scheme of a picture a third, in the concatenation of acts that constitute a purpose a fourth. But in all these cases there is an ordered whole consisting perhaps of physical parts, perhaps of successive acts or events, and in all, whether by the operation of one element on another, or by two or more elements acting in conjunction, the whole is formed, or maintained, or modified, as the case may be. ${ }^{2}$

We have spoken of consciousness as the special organ of correlation. But we must distinguish between the correlation which is effected in consciousness and the correlation which is effected by consciousness. Consciousness, at any rate in its more developed phases, has before it at any moment many elements. Among these it moves selectively, bringing into a connected whole those which stand in some definite relation to one another and to its dominant interest. This is a correlation effected within the conscious area, though it may have the effect of bringing within that area elements which would otherwise not be there. Thus I grasp certain visual and tangible data and recognise a book on psychology, which, operating on the prevailing train of interest, reminds me of a passage on a certain page which I can hunt up. The joint function which the several elements combine to form is, say, the judgment that a reference that I need will be found somewhere in Chapter X. of the book before me. This is correlation in consciousness. But this act has wider bearings. It is a step in the train

${ }^{1}$ It must be borne in mind that an organic whole is maintained not against all modification, but constantly through some modification.

${ }^{2}$ As a matter of terminology, the elements may be described as correlated with one another in subservience to the result or as correlated collectively or individually with the result. 
of actions by which I bring my past reading to bear on the whole task of writing this book. It helps to correlate a long train of experience with a long series of co-ordinated activities. This is a correlation effected by that and other acts of consciousness together. Now the whole of both trains may also be grasped by consciousness more or less adequately in a single act and in that sense become a correlation in consciousness. But in the first place, that will be another act of consciousness quite distinct from the first and more comprehensive; in the second place, a mind capable of the lesser, simpler synthesis might not be capable of the wider one, so that it might build without ever knowing what it is building or reviewing what it has built. The elements correlated in consciousness then do not necessarily coincide with the factors of life correlated by consciousness, and in comparing different phases of correlation we must take account both of what goes on within the conscious area and of what is effected thereby. Again, if the two things do coincide, it may be only the correlation effected by consciousness that is susceptible of proof. We have no direct knowledge of that which passes in the mind of another. We judge analogically on the basis of our own experience and of the behaviour of others. In the case of animals, their behaviour differs so far from the human as to throw a shadow of doubt on all interpretations of what is actually passing in the animal mind. The solid basis of our argument is always the correlation which the mind actually effects. We find, for example, certain external stimuli affecting the organism. We find subsequently a certain modification of behaviour conducing to a result beneficial to the organism and bearing a definable relation to the stimuli. That in such a case the effects of certain experiences are so brought into relation by forces acting within the organism as to conduce to its benefit, is then a hypothesis susceptible of the ordinary methods of inductive proof or disproof, and the result is independent of any theory of the precise mechanism by which the correlation is effected. Generally, then, our problem is to distinguish forms of correlation according to the data which may enter into them in each case and the use made of 
these data. So far as we discover exact correspondence with any human function, e.g. consciousness, we shall not hesitate to ascribe the same mechanism to an animal. But this is a secondary inference always dependent on the establishment of a definite mode of correlation, always open to an element of doubt, at any rate as to details, and fortunately of quite subordinate importance to our general purpose. The reader must understand generally that where we use terms involving consciousness of an animal we mean to allege a function corresponding in its causes and effects to the function in man described by those terms. Though we may reasonably presume such a function to be in itself similar to a conscious process of our own, this presumption will not form a premise in our reasoning.

Lastly, it must be remarked that correlation in order to be fully understood has often to be viewed under more than one aspect. Here is a series of acts directed to a particular end. We may describe the way in which they are correlated for that purpose without looking beyond the acts themselves. But if we ask how the adaptation came about we may have to look to the past experience of the individual and even to that of the race. This is another aspect of correlation. It is through the peculiar character of the organism which preserves the effect of the past that the adaptation in question has come about. The past may be conceived as acting on the present in the sense that it is represented by certain internal forces which co-operate with present conditions to produce the given adaptation. We may speak of this within the terms of our definition as a correlation of the present and past, and the exact way in which past and present are correlated is one of the distinctive marks of a given stage or form of activity. Again, while all correlation is directed to the future in the sense that it is such as to produce a result of interest to the organism, what that future is, how far it extends, and generally how the present activity is correlated with it, are questions on which a great deal turns. Thus at each stage, whether we are examining what passes in consciousness or what is effected by consciousness, we shall have always to ask what precisely is the nature of the correlation. 
between the present act and the past, on the one hand, and the future on the other.

2. We deal with conscious processes throughout then in their capacity of correlating functions. But, further, though consciousness is the special organ of correlation, it is not even in man the sole method, and among lower organisms it-or its analogue-is not even the most important method. Our task then is not merely to analyse consciousness, but, more generally, to consider the kind of correlation that we find in the acts of organisms and in particular of men. We shall distinguish responses to the environment from this point of view. There will be first action in which no correlation appears, and then among correlated activities there will be different types or species of correlation. These will be found to differ $(a)$ according to the function of the present organic condition, and particularly to the way in which this condition is expressed in conscious effort, $(b)$ according to the part played by the living experience of the individual or by causes acting on the individual only through his experience as compared with the part played by the hereditary structure. We shall distinguish accordingly correlation resting on heredity, correlation resting on co-present conditions and correlation resting on past experience, and of each we shall find distinct species.

\section{A. NON-CORRELATED OR SPORADIC ACTION.}

3. A man subject to nervous shock and unable to control himself throws his limbs about, twists his body, utters cries, or swears meaninglessly. The excessive excitement produces general muscular contractions which have no specific functions, though they may give indirect relief by draining off pent-up stores of nervous energy. If we are dealing with any obstacle which we utterly fail to understand, action, particularly if we lose our heads, tends to relapse into this convulsive meaningless form. In such a case we touch bottom. We sink to the lowest level at which not only all that is rational in action, but all that is adaptive and useful is eliminated. We have a general discharge 
of excitement permeating all available channels without differentiation. In the higher organisms such channels are clearly marked, and the excitement will always run mainly along nerve fibres and will affect the tissues innervated, not only the muscles but of course the viscera. Indeed, the imperative need felt for muscular action in cases of powerful emotion probably arises from the necessity of relieving heart, lungs, stomach and bowels from the strain which otherwise falls exclusively on them and produces intense discomfort and possibly serious ill effects. In the lowest organisms the channels are less distinct, and excitements sometimes propagate themselves through the whole mass of protoplasm. If there were no channels at all there would be a wholly undifferentiated discharge, yielding a quite random reaction to any and every kind of stimulus. Whether such complete absence of differentiation has ever existed may be questioned. But we can recognise the existence of discharges which are undifferentiated in the sense that they permeate all available channels indifferently. Such discharges occur in man mainly where purposive action fails or where the excitement is too strong to be readily contained, but if neither purpose nor any other form of adaptive correlation existed they would be normal. Undifferentiated discharges with the random actions to which they give rise are what remain when all correlation is taken away. Conversely, we may regard them as the material out of which those forms of reaction are selected which tend to secure the vital needs of the organism.

\section{B. CORRELATED ACTION.}

\section{Correlation by Heredity.}

\section{(I) Structural Activity.}

Uncorrelated action, it need hardly be said, is the exception in animal life. In all living beings normal behaviour runs on lines which roughly or accurately, in broad sweep or in detailed precision, coincide with the temporary or permanent requirements of the organism. At the basis of this adjustment lie the modes of action 
which depend directly on the physical structure and are therefore part of the hereditary equipment. These actions may occur in response to external stimuli, and as such we shall meet them again in the next section under the name of reflexes, or they may proceed from internal forces maintaining by their interaction a rhythm of change. In the latter case, however, which we take first, the structural activity may often be increased, arrested or modified by external events and it may need something external to work upon. It is not therefore easy in practice to keep the two types distinct. Digestion is a process determined by the correlated action of a number of internal forces, but that digestion may begin there must of course be food taken in from without and the character of the food will affect details of the digestive process. So for respiration there must be an atmosphere, and the amount of oxygen in the atmosphere will affect the respiratory process, and through it the details of the circulation which is otherwise much nearer to a true 'automatic' function. Still, among ourselves, the vital processes as a whole are determined mainly by the interacting forces ${ }^{1}$ involved in the structure of heart, lungs, alimentary canal and nervous system, and form together the going concern, the self-maintaining process which is life. Far from being purely dependent on external things to set it going, if this process does not meet with the elements of the environment appropriate to it, e.g. oxygen or food, it sets up cravings and ultimately movements of the entire body which tend to supply the deficiency. These movements, again, are movements of limbs, arms and legs, hands and feet, determined in type by their structure. So, though the precise direction of the movement may differ indefinitely according to circumstances, there is even for highly developed behaviour a generic basis in the physical organisation which is part of the hereditary equipment.

Such type reactions are readily verifiable in the lowest

${ }^{1}$ It must be borne in mind that these forces may be set in motion by internal stimuli, and in that sense be reflex. This, however, would not destroy their independence of external stimuli which is for the moment in question. 
animals, and it is possible for a good observer to give a fairly complete inventory of the 'action system' of a Rhizopod or an Infusorian. The animal is in constant movement of the parts or the whole. The movements are not dependent on any special stimuli. They go on ' of themselves' under normal conditions, though they are affected in detail by the temporary state of the animal, e.g. by emptiness or satiety. Finally, they serve the simple life-needs, absorbing food, sometimes (not always) rejecting unsuitable matter, avoiding harmful objects, and (principally by the indirect method of avoiding other regions) guiding the animal to a suitable environment. In the successful maintenance of this behaviour there is often need for a special combination of particular actions, and here there is room for a certain variation from case to case. But the elements of the combination are always easily recognisable type reactions, the beat of cilia, movements of the body on its axis, contractions to this side or that, or whatever it be. There is always a need for definite responses to certain stimuli if these occur, but the evidence is clear that the normal activity is not merely a series of responses to special stimuli, but the outcome of the internal forces of the organism, that is to say, of the congenital structure. $^{1}$ As such we may speak of it as inherent structural activity, ${ }^{2}$ and we may lay it down that the simplest and most general form of correlation in behaviour is the broad adaptation of the lines of action to the general needs of life effected by the congenital structure in accordance with its internal forces. The cause of this correlation according to ordinary biological theory is inheritance from generations whose individuals survived or perished in proportion as their structure was well or ill adapted to life conditions. The degree of correlation thus determined

I The remarks of Loeb and others (Sixth International Congress of Psychology, 1910) do not so much as touch the facts reported by Jennings in his masterly Behaviour of the Lower Organisms (1906).

2 The term 'structural activity' would, as will be seen immediately, include the reflex. The qualification 'inherent' distinguishes actions or those elements in action which depend on internal forces from those requiring a special stimulus to set them going. 
by the structure represents, therefore, the sum of all previous adaptations of a successful kind.

\section{(2) Reflex Action.}

But life cannot depend on internal forces alone. The organism is constantly meeting changes in the environment, and it must be somehow fitted to deal with these or it will perish. The structure must respond to changes as to a stimulus, and the response must be one normally suited to the requirements of the organism in relation to the stimulus.

The most elementary form of such response is known as a Reflex act. It is one in which the stimulus of an external object calls forth a uniform response on the part of an organic structure. ${ }^{1}$ Thus the touch of something sharp or hot sets up muscular contractions or results in the withdrawal of the limb. The contact of a crumb with the windpipe induces a cough, a touch on the pseudopodium or limb of protoplasm which a rhizopod puts forth causes it to shrink up and withdraw. Normally the reflex act serves a perfectly intelligible function in the life of the organism-thus in all the instances given it helps to protect from possible injury. But though it serves this function it would be mistaken to infer that it is performed because it serves it. This would be true at best only in a very roundabout sense which we shall presently consider. We have first to note that the precise differentia of the reflex is its unintelligent and quasi-mechanical character.

To begin with, consciousness plays no essential part in it. I am conscious of sneezing, but the consciousness is here a mere effect, and the sneeze carries itself out automatically and even against my will. I blink without knowing it, and cannot help blinking even when I know that there is no danger to my eyes. The reflex may even be injurious, for it is adapted only to the normal, and yet it may be difficult or impossible to control it, and so the

${ }^{1}$ The reflex may be inhibited or modified by other parts of the organic structure. But as long as we have evidence that the tendency to react belongs to the structure as such in relation to the stimulus as such we can call the response a reflex. $V$. Sherrington, The Integrative Action of the Nervous System, p. 7 seq. 
smallpox patient has to be forcibly prevented from scratching himself. The reflex in short is not like a purposive act directly adapted to the circumstances in which it is performed in such wise as to secure a specific end. It is the result of a preformed structure adapted in general to secure a result of a certain kind in response to a stimulus of a certain kind. The result is normally beneficial, but not necessarily so, and no provision is made within the limits of the reflex structure for deviations from the ordinary type. If we ask how the structure has arisen the answer on the well-known biological lines is the same as that proposed for inherent structural activity. It was through small variations, each of which was upon the whole beneficial to its possessor. The general suitability of the reflex response to the needs of the species is thus the condition of its existence, but its actual suitability in any particular case where it is performed has nothing to do with its performance. It is adapted to needs though not at any time determined purposively by the needs which it subserves. We may express the distinction by calling it Adaptive and not purposive, and we observe that in such adaptive responses, while there is a certain correlation between response and requirement, ( $\mathrm{I}$ ) this correlation is general, assigning a definite type of action to a definite type of stimulus without provision for variation of circumstances, (2) it is sensory, affecting only responses to a definite present sense-stimulus, (3) it is effected entirely outside the sphere of conscious operation, and (4) it comes about slowly and indirectly through the massive operation of generations of ancestral experience. Such, in fact, is the general character of action which is not purposive but adaptive, and is determined not by the relation of the present to the future but entirely by the cumulative and indirect effects of the past. For, mutatis mutandis, what has been said is probably applicable in equal measure to structural activity. It is the character of type-reaction in general, i.e. of all correlation so far as fixed by structure under the conditions of heredity. 
II. Correlation by Co-existent Conditions.

(I) Equilibration.

It would however give a false impression of the operation of either form of type-reaction to speak of them without qualification as unvarying. In both relations we constantly find that the condition of the organism, concomitant stimuli and the relation of the organism to other things have their effect, controlling, limiting, possibly suppressing a reaction or adjusting one type-reaction to another in a combination or a series by which a certain result is obtained. For instance, an infusorian as it swims encounters alkaline matter. It starts back a little way, reversing the movement of its cilia. This may be taken as a reflex. But it is succeeded by a typical exploratory motion which ends in the animal's moving forward at a different angle. If the new motion again brings it into contact with the alkali the process is repeated and it continues until a direction is reached which takes it from the alkali altogether, when these reactions cease and the ordinary forward movement proceeds. Putting many such instances together we may say that there is for the organism a certain condition in which its ordinary structural activities go equably forward. Any disturbance of their equilibrium is a stimulus to reactions which continue until the equilibrium is restored. This may be achieved by a normal sequence of reactions, but if not there will be some variation from the normal, perhaps some suitable modification, perhaps merely heightened and continued activity-continued that is, until the disturbance begins to paralyse the powers of the organism. In higher stages we recognise this condition without ambiguity as one of effort. At lowest, we must regard it as one in which the co-present organic conditions modify the type-reactions, repeating or combining them in such ways as remove a disturbance. The action tending to remove the trouble is chosen out of many possibilities, and if it does not succeed the animal persists till relief is obtained. On the other hand, as soon as equilibrium is gained the "efforts" cease, and the normal 
type of activity is restored. Among ourselves such efforts, often random enough, are guided by some pressing discomfort, and the equilibrium is for us comfort. Whether we can always impute an analogous consciousness when we see similar behaviour may be matter of controversy. What is clear is that here we have actions directed to a certain result and something maintaining them as being so directed. This we shall see is at least the germ of effort and purpose. We may call it conation, defining conation generically as action dependent on the difference between the existing state of the organism and some other state which it directly or indirectly tends to bring about. In the cases taken, the conation involves a correlation of the acts of the animal with the co-existent conditions external and internal in a manner tending to organic equilibrium. Correlation is no longer effected merely by heredity and the past. Whether resting on consciousness or not, it is certainly something effected by the individual in the present for itself.

\section{(2) Sensori-motor action.}

So far the governing organic activity has been considered mainly as heightening and sustaining type-reactions as long as disturbance continues. We pass next to cases in which it appears to assume a more decided function of direction. Our first illustrations of this may be drawn from human behaviour, and they may best be understood by taking a reflex as the point of departure.

A reflex response may take the shape of an action directed to, and in a sense by the object which stimulates it. A baby's fingers close automatically on a pencil brought into contact with them. Its lips suck anything with which they come into contact. A few weeks later it grasps at anything that it sees and tries to convey it to its mouth. In these cases the reflex response may be regarded as a series of muscular contractions so graded and combined as to result in a movement definitely related to the position which the stimulating object happens to occupy. There is in them, therefore, something individual. There is a certain departure from that bare generic correlation which we regarded above as characteristic of the reflex, and in pro- 
portion as the elementary contractions that make up the response are seen to be so co-ordinated as to yield the particular motion required by the circumstances of the moment - to be dominated and guided by the position and perhaps even by the motions of the stimulating object-there is something which suggests purpose rather than that blind execution of its function by a preformed structure which we took as distinguishing the reflex pure and simple. There is also something more than the mere pressure of continued disturbance maintaining an activity that tends to relieve it. There is a more definite guidance of action in relation to an external object. Now, behaviour of this sort is externally ambiguous in character and it is exceedingly hard to decide in any individual case, particularly in the animal world, how it should be classified. What we have to do here however is to distinguish types of action by virtue of the conditions involved, and for the moment we have to deal with a type which differs externally from the reflex by its nicer adjustment to outer objects.

Among ourselves responses so adjusted are almost all acquired by practice. The grasping reflex is, I believe, an exception, but it is a response of a very simple character. The action of grasping at a thing seen is not an exception. It is at first ill performed - the child ' grasps at the moon' -and is perfected with practice. The higher adaptations of this kind, e.g. the delicate adjustments required in skating, shaving, cycling, tennis playing, are formed in response to conscious purpose, but the part which purpose plays in forming them is peculiar. It stimulates us to make the effort, to persist in the face of failure, to submit to tuition. But as every learner of a new art knows, it does not serve to direct the particular grade of effort or combination of movements which actually succeeds. Success comes gradually and unconsciously. We do the thing badly many times, and begin, by a process which we cannot explain, to do it well. We keep on falling now to the right and now to the left till slowly we discover that somehow the balance is coming. Thus though conscious purpose inspires the effort it does not tell us how we shape the adjustments through which the effort succeeds, and for 
the moment it is the nature of these adjustments which we are considering. What then is the part which consciousness plays? To deal with this question let us leave the process of learning for the present, and consider first the acquired art. Here it is pretty clear from common experience that so far as we are successful in executing what we have learnt, consciousness is concentrated on the object of perception, not on the act nor on its results. The batsman concentrates his whole mind on the ball as it comes towards him, and this perception discharges automatically (i.e. by processes in which consciousness plays no direct part) the proper movements of the bat. If he 'places' the ball successfully in a direction where he had observed a gap in the field, this is the consequence of a previous observation still operating on the fringe of consciousness, but not in such a way as to impair the focussing of the percept. The motion thus seems to follow on the sense perception without the further intervention of consciousness. Conscious perception leads direct to motion, and so we speak of responses of this type as sensori-motor actions.

What is the precise function of consciousness in these cases? We have as the basis of the skilled act a structure fitted to respond to stimuli of a certain order. But a structure, as we have seen, can only be adapted to general requirements, i.e. to meet a certain type of stimulus, $A$, with a type of response $\alpha$ and a type B with a response $\beta$, the response in each case being that which is generally suitable. Now, what happens in any matter requiring much skill in the treatment is that the situations are often unique, that what is wanted is not $\alpha$ or $\beta$, but a certain combination of $\alpha$ with $\beta$, involving perhaps some grading or modification of each. The function of the close conscious attention to the precise position, distance, movement, size, etc., of the object dealt with at any moment is to combine or correlate these distinct data, to yield us the precise combination, A-B, of sense-elements which corresponds accurately to the situation as a whole. Each element in this combination discharges its appropriate motor impulse $\alpha, \beta$, but their union in consciousness effects through a machinery which does not enter into consciousness a corresponding 
modification of impulse by impulse, of $\alpha$ by $\beta$. The precise function of consciousness then in sensori-motor action is to grasp the unique combination of stimuli, each of which having its special reaction modified by the concomitant reactions, there follows a response appropriate to the unique situation as a whole.

In man all skill is acquired, and few, if any, sensor1motor adjustments are wholly innate. But among the lower animals the hereditary mechanism plays a larger part. The pecking of a chick, the spinning of a spider's web, are highly developed (though not perfect) from birth, and there is no reason to deny that such adjustments might be fully perfected by heredity. But in any such case one of two things would seem to hold. Either the adjustment must be such that every variation in the position or movement of the object to be dealt with can call out a response which will be suitable in the great majority of cases-a type response. In that event a preformed machinery can deal with the situation. Or if that is impossible, if, say, the possible movements of a prey are so individual and uncertain that no two situations will be alike, then we must infer a function capable of dealing with the novel and the individual. That will be a function identical in its operation with the conscious attention to the object which we have noted in the parallel case among ourselves. There is no difficulty in supposing this function to operate on the basis of a hereditary structure just as it operates on a structure which is modified and improved by practice. To crouch and spring are hereditary modes of action. Just how long to crouch, and when and how to spring will be determined by the keen-eyed watching of the behaviour of this particular prey. Much of the more complex activity of the lower animals may be and probably is of this type. That is, it is sensori-motor action where a hereditary structure is guided in its response by a sense-synthesis of the objects with which it has to deal.

In either case, whether it is operating upon an inherited or an acquired structure, how consciousness effects the suitable response is unknown. All that we can say about it is negative. Though it is consciousness its method is 
unconscious. We see, we watch closely, and then we strike and hit the mark. What is correlated in consciousness is a mass of percepts, the ball coming towards us, its successive movements, the ground, the position of men in the field, and some elements perhaps of our own motions in raising the bat. The result is a boundary hit, of the mechanism of which at the moment we can give no account, while if we try to attend to it, it only distracts us. It goes off in accordance with the structure furnished by heredity or by experience or by both combined, stable enough to give results of the right type, plastic enough to respond to the particular combination of impressions which consciousness effects. The function of consciousness in sensori-motor action is not to correlate the present with the past or the future, but to correlate the data of the present with one another in a way which effects a corresponding correlation of the functions of pre-existing structure, whether that structure were formed entirely by heredity or in part by experience. What is effected in consciousness is a union of sense data governing a conation. What is effected by this union is the adjustment of general tendencies to given variations in individual cases. Conversely, where we find such adjustment as a regular incident of life, we are justified in attributing it to consciousness, since consciousness is for us essentially the organ for effecting novel and unique combinations. Sensori-motor action then is probably the earliest verifiable function of consciousness, as it is certainly one of the most widespread. ${ }^{1}$

\section{(3) Instinct.}

We have conceived sensori-motor response as governed by the needs of the moment rather than the future. It

1 What sort of awareness an amoeba may have of its prey we cannot tell, but no one can read Mr. Jennings' account of an amoeba hunt without receiving the strong impression that the behaviour is of sensorimotor type. It is of course possible that analysis may ultimately resolve it into a series of type actions, in which the peculiar combination is due to the successive actions of the prey, but as it stands the evidence is all the other way. So far as our information goes then consciousness must be carried down to the lowest animal types. 
may serve the future, however, and that for one of two reasons. First, the mind may be dominated by a purpose. In that case, while the purpose lasts there will be satisfaction only in that which tends to forward it, and dissatisfaction with everything else. It fixes the feeling tone ${ }^{1}$ which constitutes the co-present organic condition dominating each adjustment from moment to moment. Thus, in the game the desire to win is present in the form of a tension, stimulating and directing each sensori-motor response. The response is guided and adjusted to the act which at any given moment relieves the tension, and as under the influence of intelligent purpose the act which relieves the tension, which satisfies, or establishes momentary equilibrium, is normally one which brings us nearer to the end, the result is that the purpose gets itself accomplished.

But without the formation of purpose it is possible that actions should be co-ordinated in series, so as to produce results of importance to the organism. This brings us to the second method in which sensori-motor response may serve the future. Just as the hereditary structure may determine a reflex response, which performs a function without intelligence or purpose, so it may determine a tension of feeling guiding a train of sensori-motor actsand indeed of structural and reflex acts along with themand persisting till a result of importance to the organism is attained. Trains of action so determined are generically instincts. We may conceive that where there is a welldeveloped instinct, but little or no intelligence, the train of action is determined by a tension, which at any given point is satisfied only by a performance which falls in with the course leading up to the final accomplishment of the result, and by no other. The solitary wasp dragging a spider to its hole does not act altogether mechanically, nor altogether intelligently. But it is not satisfied till it gets the spider into the hole. That result, and no other, relieves the tension. Where intelligence arises within the sphere of instinct, it probably takes short views at the outset, and

1 On the assumptions involved in postulating feeling a few words are said in another connection. See below, Ch. V. §3, p. 64 . 
aims at near results, which will relieve the tension and so satisfy. From these it advances step by step till it grasps the end of the instinct, which then becomes suffused with purpose.

Among the higher animals, but particularly among the most developed insects, there are long trains of intricately adjusted actions, which can be conclusively shown to be independent of any intelligent apprehension of their ultimate end, though they may use a measure of dawning intelligence in the manner indicated in executing certain steps. These form the instincts proper, and their genesis is to be understood by the analogy of the reflex, i.e. as arising through the accumulated effect of small variations, each of which is serviceable to the species. In the case of the reflex, what comes about is a structure adjusted so as to respond to a sense stimulus in a manner which serves a need. In the case of the instinct, the adjustment is more complex. There is first a tension which continues or recurs until a need is met, and secondly, an adjustment which secures that this tension is at any given moment relieved by the action which under the circumstances is in the train tending to serve the need. The state of momentary equilibrium or satisfaction, that is to say, is adjusted to the appropriate combination of objects and actions. It determines that sensori-motor adjustment which is in fact required by the organic need, and as the tension is constantly revived till the need is met it governs a train of adjustments which are in the end successful. ${ }^{1}$ Instinct

1 Note here the development of conation involved in the evolution of instinct. We saw above that conation was involved in the maintenance or recovery of the optimum or equilibrium state in the presence of disturbing causes. The term was justified on the ground that the reactions were determined by the difference between the existing state of the organism and another state, which they tend to introduce-this other state being one of 'equilibrium.' In the case of instinct, the equilibrium itself is at any moment a state of tension or conation. It is a state of excitement dependent on the difference between the existing conditions of the organism, and the conditions at the time when the instinct function is complete, and through its effect on action at each moment it tends to produce the state which terminates its activity. Conation develops then from the determination of action by response to the equilibrium point, to the determination of the equilibrium point itself by reference to 
may, I think, be definitely distinguished from a compound reflex as determining sensori-motor adjustment, and as such its basis must be held to involve consciousness.

As intelligence arises and directs action to ends remote from the momentary situation, the need for the successive adjustment of feeling to each detail in a train of actions disappears, and the mind gains its freedom. Among ourselves, accordingly, we do not find such intricately dovetailed series of acts determined by heredity as appear in the animal world. But (a) we still respond to many perceptions and situations with a feeling which popular psychology calls instinctive, but which is really rather of the nature of a reflex consciousness. The feeling of resentment at an attack is as spontaneous and unreflective as the muscular movements of the counterblow. (b) We still have the permanent interests in the race preserving functions - the satisfaction of organic needs, sexual attraction, parental love. Indeed the whole vaguer mass of the social feelings are in their basis hereditary. But we are not guided merely by instinct, because the power of looking at life as a whole brings our various dispositions and tendencies into relation with one another. We are not to conceive the hereditary endowment of man as consisting in a number of separate instincts so much as in the temperament and character, that basis or background of life which, suff used as we grow up with experience, tends to determine how we will take things, how we will regard fresh experience, and weave it into the whole of our life. Reason and will are with us as hereditary as any capacity to feel or any tendency to physical or mental response to special stimulus, and it is a mistake to found human psychology on a row of separate instincts that may be variously combined. What we should emphasize rather is the element of heredity which forms the substructure of all our thought, feeling and action.

Be this as it may, we have in instinct a method which directs sensori-motor adjustment, and by so doing in-

extended vital processes. Finally, the relation between the present state and the result to which it tends may come into consciousness, and in proportion as it does so the conation becomes a purpose. 
directly effects the correlation of trains of action in subservience to vital needs. It is thus the highest form of correlation effected by heredity and co-existent conditions combined, and completes our account of the work of these factors in life. 


\section{CHAPTER V}

\section{INDIVIDUAL EXPERIENCE AND SOCIAL TRADITION}

\section{Correlation BaSed ON EXPERIENCE}

So far we have considered correlation dependent on the hereditary structure, or on the operation of present, i.e. co-existent conditions. We have now to deal with correlations arising directly or indirectly out of the past experience of the individual, and related to his future, perhaps his remote future, or to that of his society.

\section{(I) Enduring organic effects.}

Now the influence of past experience in a certain sense affects action at a very early stage. For the reaction of the organism is, of course, dependent on the organic condition at the moment, and this condition will be affected by the past. Thus the animal which has just had a full meal will in general (not always) react to food in a very different way from the animal which is hungry. Again, the animal which has begun to chase a prey has its faculties and impulses directed towards that prey in a special manner, and it may, in consequence, ignore other stimuli for the time while reacting with enhanced keenness to anything proceeding from the chase itself. In such case the past operates, but does not produce any specific types of correlation beyond those already mentioned. Its influence is chiefly interesting as evidence of a certain plasticity in the organism, which prepares the way for higher types.

\section{(2) Acclimatisation.}

Such plasticity has a more definite effect in the accommodation to special conditions, of which probably all 
organisms are in some degree capable. This accommodation, which of course is familiar in manifold directions in our own life, is also observable among Protozoa. Paramecium introduced into a weak salt solution will give the 'avoiding reaction,' and repeat it many times, but finally abandon it. If not killed by the new medium, that is to say, the animal becomes acclimatised. The efforts to escape cease, and it resumes its normal life. Often, as we know, acclimatisation will lead us to prefer the accustomed condition to that which originally suited us. In such cases there is a certain correlation based on past experience. But it is to be carefully distinguished from the correlation of actions, e.g. of means leading to some end. What it involves is a shifting of the equilibrium point, by which so many acts of the animal are governed. This point is adapted to the conditions under which the individual lives, and with this adaptation a whole attendant series of actions is, of course, modified accordingly. We might speak of acclimatisation as a correlation of the equilibrium point with the persistent conditions given in the experience of the individual.

\section{(3) Inarticulate Correlation. (a) Selective modification.}

The teaching of experience and the development of mind which is stimulated by it, if not founded on it, has as its unit a relation between two data affecting the organism. When we speak of learning by experience, or regard thought as resting on experience, this is the kind of experience that we mean, and when we trace the growth of intelligence, what we have essentially to consider is the way in which the mind apprehends or at lowest is affected by data in their relations, the kind of data that it can apprehend, and the use that it makes of them when grasped.

Probably the earliest form in which such relations affect conduct is one which is amply verified for certain Infusoria. A stentor gently touched on one side will contract upon its stalk, but will soon open out again. Touched once more, it will perhaps bend to one side, and if continually molested in this manner, it will uproot itself in pardonable dudgeon 
and swim away. That is to say, it has several ways of reacting to the stimulus and seeking equilibrium, and if one fails it tries another. But now, when it anchors itself again, it seems to have learnt something, for if again touched it does not go through the stages of contracting and of bending aside. It keeps to its more radical remedy, and moves off again. The effect is very transient, but it is there, and is amply verified for Protozoa and for several of the lower Metazoa. Observe that precisely what is learnt is not to avoid some object previously sought, or to seek some object previously indifferent. What is temporarily learnt is to prefer one type-reaction to anotherthe stronger, the more effective, but also that which the animal is normally least ready to put into action. The relation of data - which, if not apprehended by the organism, has somehow affected it-is the failure of response $A$ to remove an annoyance $B$, or conversely the success of response $C$ in this respect. It need not learn anything about the qualities or relations of $\mathrm{B}$. What has affected it is the success of one of its type-reactions in relation to $\mathrm{B}$. The stimulus $B$ becomes the basis of a different typereaction from that which it at first called forth. Correlation is effected between a stimulus and a certain type-reaction. Such correlation is observable in human action (though often in combination with higher modes of response) in the acquisition of skill, which is essentially a modification of the means by which a result is attained. We saw that in skill of any kind, though consciousness plays a part, the process of acquisition is in detail unconscious. We can now see that the general method is that of selective correlation. The too violent response lands us in a fall or punishes us with a cut. The insufficient movement ends in a bruise on the other side. If there is no bump or cut, there is at least the disappointment of failure, while the chance response that hits the mark is encouraged by the sudden joy of success. The result is that the successful movements are selected and the failures eliminated. It is reached by a process which we seek in vain to bring within our consciousness. Yet conscious experience has somehow operated to make this accuracy of execution possible. 
How it operates may be best considered in relation to a somewhat different case.

\section{(b) Assimilation.}

In acquiring skill we perfect the means to an end which is already determined. In the cases now to be considered the end itself is affected. More accurately we learn to redirect our action, to quell a response to which we were originally impelled or to meet with a definite reaction some stimulus to which we were originally indifferent.

Learning of this kind plays an important part among animals as well as among men. It is seen in its simplest form where two sensory data, as A and B, are closely related in time and where one of them is charged with feeling. The relation is particularly clear and simple in its operation where the first datum $A$ is a stimulus setting a hereditary mechanism to work, which has B as its immediate result. Thus, in a familiar observation of Mr. Lloyd Morgan, a chick sees a small bit of orange peel on the ground (A). It pecks at it, and experiences a taste (B) which is apparently unpleasant ; for the peel is dropped, and thereafter, or perhaps after one or two more experiments, orange peel is avoided. The pecking impulse is in relation to these objects inhibited. Now, in speaking of an 'unpleasant taste' we are inferring from our own consciousness a feeling in the chick which it may be difficult to verify. But we are not concerned for the moment with the inner character of the process by which correlation is effected in any particular species of animal. It is certain that among ourselves an experienced feeling ${ }^{1}$ is the operative element in cases of the kind, and in accordance with the principle laid down above, in describing animal behaviour we use terms involving such consciousness and such only as would be essential with the parallel case among men. In the human mind much higher factors may at any time co-operate, even in the simplest act, while we may have sufficient evidence for denying these factors to the animal. In such a case the

1 Meaning by the term here and throughout this discussion the entire psycho-physical process in which feeling is involved. The inherent causal efficacy of the psychical is not assumed. 
evidence of animal behaviour becomes especially valuable as serving definitely to show how far the simpler factors will carry us, and thereby to mark off lower from higher stages of correlation.

In the case of the chick then we may, on the analogy of the human child which begins to eat something nasty, then relinquishes and subsequently avoids it, impute the change to the experience of an unpleasant feeling. How then are we to describe what has happened? A stimulus $A_{1}$, exciting a movement, leads to the unpleasant experience $B_{1}$. Henceforward the reaction is modified. Similar stimuli $A_{2}, A_{3}$ no longer prompt to the same motion. Clearly the basis of this change is the relation $\mathrm{A}-\mathrm{B}$ as experienced in the case $A_{1}-B_{1}$, and one way of explaining the process would be to say that the relation A-B being once apprehended is inferred in any new case where $A$ is found. In this instance this would amount to saying that the chick connected a certain bright yellow appearance in an object with an unpleasant taste, and thus formed a perception, and on the basis of perception an idea of orange peel as yellow, peckable and nasty. There are reasons for denying any such power on the part of the chick which it is not necessary to examine in detail, but which amount to this-that if the chick had such power we should expect him to be capable of many inferences and manipulations of experience of which he is in fact incapable. It is however clear that the feeling $B_{1}$, which quells the original response $A_{1}$, has some lasting effect. In the end this effect is the same as would be produced by an apprehension of the relation A-B. But we do not suppose this apprehension to be formed. The relation then must affect consciousness without being present to consciousness. The response is correlated with its result, but correlated by some less direct method. How are we to understand this correlation? We could only answer this question adequately if we knew how it is that modifications of the mind or of the physical organism are rendered permanent. Confining ourselves to the facts that we know, what we can say is this. The painful or unpleasant experience $B_{1}$ tends to quell the reaction to $A_{1}$, and the effect persists, in this sense, that in the future similar 
reactions are more readily quelled till a point comes at which they wholly surcease. In fact the whole process may, especially among ourselves, be achieved by a single sharp experience. The psycho-physical tissue is so modified by the wave of inhibition once set up that the original reactive tendency is altogether held in check. It is not however the original inhibition which persists. That is a temporary state, which having once occurred is past, like any other event. Nor is it true to say that it is 'revived,' for by degrees, if not at once, the necessity for inhibition disappears, and a new response arises, which avoids the unpleasant object. What comes about then is a permanent modification of the psycho-physical structure, which gives directly the response ${ }^{1}$ at first reached only through the reaction of feeling.

Thus the sense-stimulus comes to act as though it were infected or charged with the feeling that is at first a mere consequence of the reaction. And this infection corresponds to something which we actually find in consciousness. The eatable that has a nauseous taste, unless there is a counteracting factor of considerable strength, will come to look nauseous. Its appearance to the eye is 'complicated' with an element of unpleasantness, charged with disagreeable character. When the ground of this unpleasantness is set out it becomes the taste of the thing, which for me, as I merely look at it, is an idea, and an idea distinct from my present perception, but this separation is effected at a higher grade of consciousness. Before any idea distinct from sense perception is formed, the sensorimotor excitement is qualified by feelings which do not originally form part of it, but which come to do so as the result of the antecedent experience of similar sensations and of the attendant response and feeling. Thus on the psychical side the excitement $A$ takes on itself in our

I If the object is simply ignored it may be said that there is no response at all. There must, however, have been a psycho-physical change perfectly comparable to that which brings about a definite movement of avoidance, and the negative result (e.g. that the orange peel is not eaten) corresponds to that which is 'at first reached only through the reaction of feeling.' 
consciousness, or 'assimilates' something of the character of $\mathrm{B}$, and we may infer that on the physical side a corresponding modification occurs. ${ }^{1}$ Thus assimilation effects in consciousness the union of a sensory excitement with a feeling originally foreign to it. This feeling determines a response which is in general satisfactory. Hence we may say that through assimilation the elements of an action are correlated with its result. But though this relation is effected by consciousness, it is not itself present as an object to consciousness. It is an underlying fact noted by the observer, but only brought into consciousness at a higher stage. Again, the new adjustment being based on past experience, assimilation may be said to correlate the present with a past situation in the service of the future. The correlation of the successive experiences of the individual is, in fact, precisely the addition made at this stage to correlation by heredity and by co-present conditions. But this relation, again, is not an object of consciousness, for there is as yet no idea of past or future. Thus assimilation is a union of elements in consciousness based on relations that do not enter consciousness, effecting correlations that do not enter consciousness. The modified sensory excitement is the product of a body of experience, stimulus, response, and feeling, acting in a mass. The elements of this mass are not sorted out in consciousness, nor can each be correlated as such with some element of a subsequent experience, as we shall see that it may be at a higher stage. Each acts indirectly as a contributory element in the massed effect, not articulately as a separate datum determining its particular part of the response. We may express this by saying that we have here a massive or inarticulate correlation of successive experiences.

We may assume that the process involved in the selective correlation of response, as in the acquisition of skill, does not involve more of consciousness or of articulate correlation than this. Probably it involves less. Whether there is a distinction of principle cutting deep into the nature of

1 This has been well brought out by Professor Holmes, 'Pleasure, Pain and the beginning of Intelligence,' Journal of Neurology and Psychology, April, 1910. 
the organism is not clear to me. ${ }^{1}$ But at all events the negative description - Inarticulate - will apply to both, and what is said later of the general effects of this method of correlation may be taken as a rule to cover both cases. Of assimilation proper the simplest case is furnished by such inhibitions of original impulse as have been described. But there are others probably of the same generic type though they are more advanced, and at least in their highest development prepare the transition to a further method. For example, a content $A$, the sound of a bell, which is originally indifferent, proves to be the beginning of a short continuous train of events culminating in the excitement of dinner (B), and $\mathrm{A}$ in consequence becomes by slow or rapid steps charged with the interest of $B$. By this method the random efforts of an animal may lead to useful habits. It may react to $A$ at first in many vague and useless ways. But one reaction gives $B$. This reaction, after one or many repetitions, is preferred. All the others get the feelingtone of failure, one alone gets that of success, and so in time $\mathrm{A}$ comes at once to prompt the right reaction. This is the method of Trial and Error, which has been shown to have great importance in the 'learning' of animals.

But among ourselves B need not in all cases be a feeling. Any element entering habitually into the same field of consciousness with A may come to colour A with its own nature. Any data that frequently impinge on one another in our consciousness may become so bound up that to our sense-apprehension one stands for all the rest. Such is the character of perception as distinguished from mere sensation, of Recognition, and of all the operations in which we detect what we call an unconscious inference. This name is inappropriate only if it suggests that there is in consciousness any transition from premise to conclusion. In reality I see that wall as a solid object built of brick, though in point of fact I could not by vision alone adequately test its solidity, to say nothing of its composition. But many

1 It may be noted that among Protozoa the evidence for selective response is clear, and that for true assimilation very doubtful. Among Coelenterata, however, true cases of the reversal of a response to stimulus are reported. (See Washburn, The Animal Mind, p. 214 .) 
experiences of touch, resistance and so forth have from infancy been operating upon me in such a way that the apprehension of a red extended surface is filled out automatically with elements that make it into the perception of a wall built of bricks and mortar which I cannot push over or walk through. Logically when I see a little figure running to meet me, and discern it to be my child, I am inferring from a patch of colour quite a fabric of potential conclusions. Psychologically what has happened is that all the meaning that the term ' $m y$ child' has for me has got itself incorporated with that vision. The optical sensation is charged with possibilities of meaning, any one of which may be developed into ideas or acts according to the interest of the moment.

In action the characteristic product of assimilation is Habit. Just as the hereditary structure produces reflex responses to sensory stimuli of a definite type, so assimilation produces reactions which are the same for all stimuli of a class. Correlation of this order does not lend itself readily either to correction or to accurate discrimination of essentially different cases, and where we find habits slowly formed and obstinately adhered to we may refer them to Assimilation. The reason is quite intelligible. The process consists in the modification of the excitement corresponding to $\mathrm{A}$ by its assimilation of the character of $\mathrm{B}$. This may take many repetitions to render it permanent, and once permanent it is a structural change which similarly requires much effort to undo. For the same reason the modification easily extends itself to $a$ and $\alpha$, which to the senses resemble $A$, but have quite different effects, while it fails to affect $A_{1}$, which to a superficial view differs from $A$, but in reality has substantially the same effect. Habit, in short, like the reflex, is of the nature of a structure built up to suit the simple and the normal, and outside that range failing disastrously.

In the human mind Assimilation is responsible for more than Habit. Past experience operates unconsciously on the highest and most developed as on the most elementary mental products. Our experience in the mass goes to shape our thinking, to suggest one train of thought or 
exclude another, to determine the way in which we take things. It fuses with the hereditary substratum, and makes of it a new, though more plastic and modifiable, structure, which operates for the most part altogether without self-consciousness. Inarticulate correlation thus operates with elements that arise at higher stages of development than its own, and helps to form the permanent background of our thinking, our purposes, our emotions. But if we wish to understand its action and measure its achievement as such we must strip away all these higher elements. We must reckon only what it adds to the work of reflex and sensori-motor action. So considered, and taking its two specific forms together, its function is to build up the habits and the skill, which form the basis of sensori-motor action, so far as this is not already determined by heredity. The essential new fact which it introduces is that the experience of the individual co-operates with that of the race in determining action. Past and future are correlated, but the correlation is 'massive' and inarticulate. It is effected by consciousness, but not in consciousness, and the result is a structure which yields type-reactions, not a purpose which can adapt action at need to every variation of circumstances which bears upon the end.

\section{(4) Articulate Correlation-Co-ordination of Concrete Elements.}

Thus far we have supposed the reaction upon a stimulus $A$ to be modified by the effects of the attendant experience $B$, and have shown how that might happen without any express correlation or co-ordination of $\mathrm{A}$ and $\mathrm{B}$. Let us now suppose that this correlation occurs. The individual now has an experience which we may write A-B. It is an experience of two related elements. $A$ is an object to the right of B, or it is an event followed by B. E.g. I want my book, I remember that I left it on the table to the right of the door in my bedroom. To get it I have to go indoors, upstairs, and in at the second door on the left. Here there is a quite explicit reference to a set of related elements. These elements in their relations have entered into my experience, and as such form the basis of my present action, 
in which the relation of each step to the end is also explicitly present to me. The correlation of elements falls within consciousness. It does not merely affect consciousness from outside. It is part of the explicit content. In particular the relation of my act to its result is clear to my consciousness. There is in fact a correlation on the one hand of perceptual data, the space relations of book, table, room, etc., and on the other of practical means and ends, the movements necessary to get the book, and the correlation of practical means and ends is based on the correlation of perceptual data.

In such correlation of means and ends we are said to act intentionally or with purpose, and the end is held in ordinary thought to determine the act. This at once raises the question, how and in what sense can a future event, no matter how near, be conceived as actually going to determine, to cause, the act which brings it about? As to the proximate means common language has its answer. The effect of my act determines me through the idea which I form of it. The idea is a state or act referring to something not as such present, and when I form such an idea and act upon it, I act with purpose, and when I act with purpose I do so either desiring or resolving to obtain the end. We shall see that resolve, so far as it is distinct from Desire, involves the elements that constitute desire (and aversion) and more. We may therefore confine ourselves for the present to Desire, and define it as an idea of something not yet real, charged with the feeling-tone prompting to such actions as will make it real. Action of this kind therefore involves Purpose in the form of Desire, and these involve Ideas, and as the ideas are of co-ordinated elements and ideal elements presented in co-ordination form the content of Judgments, we may say that the judgment also is involved. As the judgment of the present case is based on or determined by a past judgment ${ }^{1}$ we must admit that it is inferential, but the inferential process is not as yet necessarily explicit-that is the common elements connecting premise and conclusion do not form distinct contents

${ }^{1}$ I mean, e.g. that my belief that the book is in my room is based on the remembrance that I left it there. 
of consciousness. Indeed the premise itself may fail to be explicit. Without being definitely remembered a past experience may operate unconsciously to supply the relevant idea at the moment of action.' Explicit inference is not therefore included among the mental processes necessarily involved.

It will be said that, granted this prima facie analysis, it is still absurd to talk of an actual determination of present by future. What has happened is that the course of experience has generated in the individual the state of tension called Desire. There is in this state a mental reference to something future, of a line of action leading up to the effect and blended therewith an impulse to move along this line of action. But though this is a forward-looking state, still it is a presently-existing state which has grown up out of the past, and by its present character determines future phases. It is not determined by them, because what now is cannot be determined by relation to what will be. We shall discuss this matter more fully at a later stage, and give reasons for rejecting this view, and shall therefore allow ourselves in the meantime to stand by the ordinary way of thought which speaks of a purposive act as determined by its ends. We have only to note that the end is also determined by the purposive act, and that there is therefore a true mutual correlation of act and end.

This brings us to a further question, how do we distinguish correlation of this kind from the unconscious correlation of the previous stage? Where we have to judge by external behaviour only the distinction is by no means easy to make, and involves some of the most difficult questions of comparative psychology. Thus we ordinarily conceive a dog as begging in order to get food, i.e. intelligently, purposively. But suppose it is suggested that previous experiences of the begging posture and attendant gratifications have bred up in the dog the habit of begging

1 The fact that I left the book may operate in consciousness to engender the anticipation, 'I shall find the book there,' rather than the memoryjudgment, 'I left it there this morning.' It must be admitted that in the human mind the one judgment passes into the other, but at a less reflective level it may be otherwise. See below, p. 80 and note. 
when hungry? Suppose, that is to say, that the apparently intelligent performance is taken as a case of unconscious. correlation. How are we to meet the suggestion? How do we distinguish between the indirect effects of related experiences on the reaction to stimulus, and the direct effects of a correlation established within consciousness? The reply is that among human beings the distinction is made clear in the first place by careful attention to the contents that come before consciousness, and in the second place by the nature of the action involved. It is the difference, for example, between my perception of the wall in which, as we have seen, the experiences of solidity, etc., qualify the actual content of vision, and the explicit judgment 'That is a brick wall ' - not a painted or reflected wall which to mere vision might equally possess the 'solid' character. It is the difference between the emotion of fear which a thunderclap may produce and the explicit judgment that in a moment we may be struck dead. In action the differences are still more marked. The habits bred by unconscious correlation are habits of type-reaction to typestimuli. True, as we have seen, these reactions may be graded and refined in detail to meet the variation of individual cases. But all these cases fall under a type, which as a type produces a generic form of motor reaction and attendant feeling. Now in the search for my book there is nothing of this. The need of a book in general or of that particular book does not discharge in me the set of motions. that take me from wherever I happen to be to my bedroom. The whole case is unique, and its uniqueness depends on the particular concrete relations of the book which fall within my explicit memory or at any rate within explicit past experience. What we achieve at the present stage then is the appreciation of the different relations in which things stand to one another, even though these relations are not present to perception, together with the power of so using them as to gain our ends. The concrete circumstances in which any living being is placed are always varying. Any element in its surroundings stands in many relations, and any one of these relations, seen or unseen, near or remote, may in fact be relevant to the life and pur- 
poses of the individual. It may be on the whole better for the individual that it should acquire a stereotyped method of reacting to a certain element than that it should act purely at random. But it is better still that it should be able to vary its actions according to the relations in which that element stands, and this it is able to do by the conscious recognition of those relations.

The conditions of such variation are in the main two. First, the mind must be able to appreciate distinct elements in relation. $\mathrm{A}$ and $\mathrm{B}$ must not fuse or be assimilated. They must remain distinct and yet be related. Thus the sound of the bell must not merely be charged with the suggestion of dinner. It must remain a clearly-cut content on which dinner as another clear-cut content follows in time sequence. But secondly, the sequence once apprehended must somehow serve as a guide to action. At lowest this involves that where ' $A$,' say, is present as an object there is an effort to institute the sequence $\mathrm{AB}$. But $\mathrm{B}$ is not here something present. It is not an object to the senses. If there is true conscious effort to bring it about there is a conscious state involving direction or reference to something not present. Such a reference generically is an idea. The emergence of ideas is a fundamental departure in the life of mind. Hitherto we have considered consciousness as concerned with objects present or given to it-expressions which we may paraphrase, but which we have not succeeded in analysing further. The mind is either merely aware of what is given or reacts upon it, seeking to enjoy it and maintain it, or to escape from it, be rid of it. These are modes of conation, the first of which is barely distinguishable from the feeling of pleasure, while the second is evidenced in a series of definite efforts or conations. Now with the emergence of ideas we get an explicit reference to something which is not present at all, and which serves from the outset to direct conation to the production of something that is not yet, but can be definitely anticipated. How does this transition effect itself?

Let us note in the first place that in perception we have a mental act which may be said to occupy an intermediate 
position. For perception is essentially a judgment asserting something given. Yet its assertion contains somehow more than is given, for the perceptual judgment may be wholly or partially false, whereas what is given qua given is simply fact. Perception may be said generally to assert something given as having its existence in some definite spatial relation, and it is in regard to this spatial relation that error may arise, for instance, in any case of illusion. This reference effected by perception arises from the action of the given on a mind possessing (I) certain definite tendencies to correlate its experiences in certain ways, and (2) a certain antecedent experience which it brings to bear on the present from moment to moment. Since the experience is incomplete and the tendencies imperfect as agencies of absolute truth the perceptual judgment may be in varying degrees inaccurate or false. Perception then is not a mere acceptance or awareness of what is given, but an interpretation of the given which refers it to a definite position in space and time. Nevertheless perception is a judgment about the given, and thus falls on that side of the line of which we have already taken account, whereas any explicit reference to that which is not given falls on the other side within the world of ideas and ideal-judgments. We have thus to ask how ideas may be conceived as emerging in a mind which is at first concerned with the given.

The idea is not, as the early empiricists supposed, simply a faint revival of the past experience, for it is unlike the experience which it is supposed to copy. The image which may arise in my mind now of a place which I saw last year might be explained as such a revival. But my memory judgment, 'I was there last year about this time,' is an act of quite different character from the perceptions which I experienced last year. It is an act of reference to them or to their objects as something belonging to my past. Similarly, an anticipation which guides my efforts is an act of reference to a possible future. 'Revival' alone will not explain the genesis of this type of reference, but revival operating on other mental elements may help us. The general history of mental development suggests our looking for such an element on the side of conation. We may 
pretty confidently assume that ideas first arise as subsidiary to conation and as directing it. Now we have seen conation in its earliest forms as a spasmodic activity excited by discomfort and continuing till the discomfort is removed. But in the most developed forms of sensori-motor action we have already reached a more definite species of effort than this. Particularly as the 'distance receptors,' i.e. the senses of sight, hearing and smell, evolve, we have action directed definitely to certain distant objects. Such effort again we have seen will be confirmed by an agreeable experience, and in this we have a form of 'revival.' The general character of this revival is that a conation involving perhaps an ordered series of actions may be set going by a stimulus which has previously been a starting point of a successful effort, i.e. one that has had agreeable results. Now let us suppose revival to operate on a mind capable of perceiving three objects $A, B, C$ in definite space and time relations, $\mathrm{C}$ being something desirable, e.g. food. If the three objects are present to the senses, the first two leading up to the third (e.g. as intervening objects in space), conation will be definitely directed to $\mathrm{C}$ via $\mathrm{A}$ and $\mathrm{B}$. Let this have happened and then let $A$ alone be given. If the animal is hungry, i.e. if there is a conational basis to go upon, A will, according to the law of revival, excite a conation corresponding to the previous one, but this was a conation definitely directed to $\mathrm{B}$ and $\mathrm{C}$ in succession as things standing in a definite relation to $A$. The animal then directs its efforts to the point where, in accordance with the first experience, $\mathrm{B}$ and $\mathrm{C}$ should be. It looks for them, or if $\mathrm{B}$ is some change which brings $\mathrm{C}$ about, sets itself to perform B and so obtain C. Its action is directed to something not given, and this appears to be the germ of a conational or practical idea. The further step required is the disengagement of the idea on the one hand from the direct conational interest, on the other from the order of past experience. This may be conceived as arising from the circumstance that any desired object will stand in relation with many different things, one of which will be relevant in one case and another in another. Without pursuing this development here we may conclude that the 
first emergence of an idea consists in the definite direction of effort to something not given, and arises from the joint operation of three conditions, articulate perception, directed conation, and the power of a stimulus to 'revive' a defined effort.

Whatever the precise genesis of the new process, what has come about is an effort consciously directed to something absent, a mental state of a new kind which is apparently due to an effect of a past perceived relation impinging on a present conation and so defining it. In the full development of this form of correlation such effort involves an anticipation. ${ }^{1}$ But if we are to so describe it we must bear in mind that the anticipatory state has its feeling-tone tending to set up the action suited to it. The effect of the development will be that action is now pivoted, not as before on A, but on B itself, and may be varied in accordance with any of the relations in which I stand at the moment and which experience suggests as likely to affect B.

Whether correlation of this order is attained by the most intelligent animals is an open question. The affirmative view might be proved if we could show one of three things, viz. (I) that animals can learn from witnessing the sequence of events or the relations of objects, and not merely by the modification of their own action by attendant feelings. E.g. if a dog sees a bolt pulled and a door opened disclosing food within and then comes to pull the bolt himself, the inference is that he has correlated a little series of events. Experiments on these lines give very varied results, and the interpretations of experimentalists differ. The question cannot be regarded as settled, but upon the whole the evidence shows that such 'learning' is excep-

${ }^{1}$ It cannot be too emphatically stated that an idea at this stage is not a general idea. It is a reference to something to come, that is, something particular. It may in the full development of this form of correlation also be a reference to a particular event in the past, but $I$ have no doubt that Miss Washburn (The Animal Mind, p. 274) is right in contending that the first function of ideas is to guide conation, that is anticipatory. We might call such ideas Images, but that involves a description of their character which is not always easy to verify and is not necessary to the bare statement of their function. That function is direction, or, as I call it, in order to bring out the generic community with other ideas, reference. 
tional, but that it does occur among apes and monkeys and probably among some other mammals. ${ }^{1}$ (2) That animals vary their action according to the individual circumstances of the case-the relations in which they stand. Thus a dog has been scolded or punished for lying on the sofa with his dirty paws. He avoids it in the presence of his master, but indulges in his absence, and pays no regard perhaps to the presence of some more easy-going member of the family. Mere habit, it may be argued, would have induced avoidance of the sofa, or perhaps of the person who struck him, and for reasons of this kind a less intelligent animal is incapable of instruction unless of the most rudimentary kind. A dog is afraid neither of the sofa nor of his master nor even of the stick, but only of a certain expressive combination of the three. It is of course possible to suggest that the dog learns by habituation to respond to that particular combination, but the explanation wears very thin when it has to be extended to account for every difference which an intelligent dog will make in dealing with different people and different things. The essence of the correlation with which we are dealing is that it guides action in any situation by reference to its special relation to the object desired, and if an animal can vary its action in accordance with such special relations, not once or twice as by happy accident, but as a regular part of its behaviour, it must be taken to have advanced beyond the stage of learning by mere habituation. (3) Evidence of conscious correlation may be derived from cases in which an animal performs a novel action as the result of relations which it has experienced. E.g. in a well-known story a dog chases a rabbit

${ }^{1}$ For monkeys, see 'Imitation in Monkeys,' by M. E. Haggerty, Journal of Comparative Neurology and Psychology (July, 1909); 'Some Mental Processes of the Rhesus Monkey,' by William T. Shepherd (Psychological Monographs, Nov., 1910). For cats, 'An Experimental Study of Imitation in Cats,' by C. S. Berry (J.C.N. and Ps., 1908). For Raccoons, 'Concerning the Intelligence of Raccoons,' by L. W. Cole (ed. 1907) cited in 'Animal Behaviour,' by H. S. Jennings (American Naturalist, March, 1908). I have not seen Mr. Cole's article, but have to thank the other writers mentioned for their monographs. Mr. Jennings in the same paper quotes from Mr. G. van T. Hamilton an experiment showing what appears as a high development of this method in a dog. But again I have not seen the original. 
which bolts for a drain; next day seeing the rabbit again it makes straight for the drain. Here there are no materials for habituation to work on. It is the perceived relation that operates. A few carefully studied instances of this sort would put the matter beyond dispute, but unfortunately evidence of the kind is from the nature of the case anecdotal, and it is not only untrustworthy in its detail but entirely lacking in study of the previous conditions, which would very often put the incident in a different light.

It must then be admitted that the question whether the animal mind reaches the stage of conscious correlation remains unsettled. In my own view the probabilities favour the affirmative answer, ${ }^{1}$ and I shall provisionally assume that this stage in mental evolution is reached before the birth of the human race. There is the more reason for this view in that language, the distinctive characteristic of humanity, the necessary instrument of human thought, the basis of the social mind, is not essential to the correlation of perceptual elements or of the practical means to near and concrete ends. Be this as it may, we have in any case a further stage in the development of Mind to be noted. Its distinctive feature is that a relation such as A-B which before only affected our attitude to $A$ now enters into consciousness. We can apprehend terms in their relations and therewith any one term in many relations. On the basis of this articulate experience we form anticipations and ideal constructions, and so far as any of these are imbued with feeling-tone we conceive desires and aversions, and shape our action thereby, i.e. we act with purpose. Articulate perception, idea and desire thus go together as the characteristics of this stage. We have in consciousness a direct correlation of distinct elements of perception on the one hand, and of means and ends on the other. Action is no

${ }^{1}$ Whether if animals do attain this method of correlation they employ the same mechanism as the human mind, i.e. particular or 'practical 'ideas, is a further question, far harder to determine. We cannot look into the animal mind, we can only ascertain at best whether its behaviour involves a function corresponding point for point with one of our own. But the precise nature of that which passes in the animal consciousness is for my purpose of much less importance than the kind of correlation which it achieves. 
longer, as in the previous stage, correlated indirectly with its result, but directly aimed at it. Nor is it based on the massed experience of the past alone, but may be correlated detail for detail with the relations of objects as they have been given in perception. We are always dealing with the perceptual surroundings or with the object of desire, and the ideal links between it and the percept of the moment. ${ }^{1}$ But in either case we have distinct elements articulately related to one another. If we conceive such correlation repeated continually without any advance beyond this plane of mental activity we shall have a ramifying co-ordination of the objects and events which make up the perceptual world of the individual, serving from time to time as a basis for the satisfaction of his desires. This world will not be present to consciousness as a system, but any part of it may operate within consciousness when it is relevant to a momentary need, and we must observe that although the objects before consciousness at each moment are particular objects, what is relevant in the guidance of action is that which is common to the existing situation and to others that are already familiar. We have a correlation of particulars based on common elements. This correlation may be called Direct (or conscious) and Articulate.

\section{(5) Correlation of Universals-Analysis and Synthesis.}

The inference from the past to the present or the future is founded on a certain community of character which unites them, and it becomes explicit, and so far rational, in proportion as this element rises into consciousness and is recognised as the basis of our proceedings. From the look of things I think a thunderstorm is coming on. This is an expectation based, let us say, on my own observations of the weather, in particular it may be of yesterday's weather.

${ }^{1}$ In the human mind there may be distinct reference to the past at this stage (i.e. without involving general ideas). That is there are true memory judgments as well as anticipations. To verify such memory judgments as distinct from efforts based on anticipation guided by past experiences seems impossible if our evidence rests on external behaviour alone. True memory as distinct from anticipatory ideas cannot therefore be securely attributed to animals. 
I may not think about the basis of the matter. I may be concerned merely with the present and I put off my walk. But if a discussion ensues I begin to analyse, I point to those clouds, remark on the heat, consider the direction of the wind and (to take the matter in a very simple form) point to the correspondence in all these details with the situation of yesterday. This is to dissect the situation as perception gives it me, to find elements common to it and to a previous situation, and to make these common elements an explicit ground of inferring a further point of resemblance. There are here the essentials of the reasoning process, the bare elements of which may be succinctly characterised. The data of perception are resolved into distinct elements of character recognised as qualifying experience (analysis), and such elements can be combined to form new wholes without any reference to the order in which they are perceived (synthesis). ${ }^{1} \quad$ Hence are formed thought constructions or concepts which take us altogether beyond the world of perception. Whither they take us, whether to a region of pure imagination or to a deeper reality than that of perceptual experience depends on the way in which they are formed. In this process the structure of the mind as shaped indirectly by racial and more directly by personal experience is necessarily the determining force, but at the outset it operates unconsciously. Inasmuch as it has been formed under the conditions governing survival, it tends in the main so to construct our thought-world as to facilitate and improve our dealings with reality. But this is only to secure a very rough and general correspondence, and how far thought actually yields truth remains a question, which is only to be solved by bringing its data, methods and results into conscious correlation. This is the work of a higher phase of development of which we shall speak presently. Meanwhile we must note certain points bearing on the evolution of the thought-world.

1 It should be understood that analysis and synthesis are not two separate processes, but rather distinguishable phases of what is essentially a single process of correlation. Where one is explicit the other will be found to be implied. Thus, what is consciously a comparison, and so a synthesis of two objects, rests, on an analysis, and conversely. 
In the first place, we must remark that from the beginning of this phase we are really passing out of the development of the individual mind strictly considered. It is of course conceivable that the process of analysis and synthesis might arise in the mind of an isolated individual, but as we know it, it is the product of communication between mind and mind, resting on and in turn facilitating the development of language. For the name of common significance involves analysis, and the significant sentence is a synthesis of elements which analysis has rendered distinct. To give a thing a name which will be understood is to select in it a character common to it and to other objects within the experience of the speaker and the hearer, ${ }^{1}$ and to say anything intelligible about a thing is to render a combination of elements in idea, which combination the words must be able to reproduce in the hearer's mind unaided by perception. Thus analyses and syntheses of perceptual experience are the basis of language, while conversely they can never go far in advance of language. The meanings which we cannot somehow express we not only fail to propagate among others, we lose them ourselves, they are fleeting impressions, lights and shadows of reality which we cannot fix and unwillingly let go. Language then - or more generally the social means of expressionforms a kind of sieve catching the expressible and letting go those elements of experience which it cannot render. The degree of adequacy with which it can express meanings is accordingly of the first importance in the development of human thought.

The common elements which we find in experience and which serve as a basis of interconnection between its parts fall generally into two categories. There is in the first place community of character, or Resemblance, which lies at the basis of all generalisation. The resemblance may be loose and vague or it may be precise, and the advance of exact thought consists on one side in analysing loose

1 A proper name might seem to be an exception, but is not. The individual is a continuous being running through my experience, recurrent in many of my experiences and common to them, and also, if he name signifies anything to you, common to you and me. 
and vague similarity into elements of exact resemblance and definite difference, concepts, or concept-elements which are the true units of science. As such elements become distinct we arrive at propositions which are true of them as such, and thus reach the explicit conception of the universal and the necessary. These relations are shot through the varying movement of our experience connecting things most remote in space and time by underlying affinity of character, and so we may think of this form of correlation as a 'cross-correlation' in that it cuts across the order in which experience comes to us. Regarded as a method of dealing with that experience, what it enables us to do is to grasp it in masses, grouped by affinity of character and consequent necessity of interrelation. It forms our world into Classes of which we can take a comprehensive view, by reference to which we can judge new cases, and with the aid of which we form general rules of action.

In the second place, the basis of interconnection may be continuity of existence, i.e. the continuity of an individual passing through various phases or presenting numerous qualities in simultaneity and succession. Here again the concept is a basis of correlation between an indefinitely great number of concrete elements of experience, and when it is brought to bear upon action serves to correlate the act of the moment with permanent interests and general principles. By its means the individual consciousness grasps the continuity running through its experience and projects it into the future. It becomes conscious of Self-for the self, as remarked above, is the element of continuity running through the acts of consciousness - and at the same time and by the same methods aware of other persons, and of the social groupings which they form. It can focus its own experience in generalisations, and learn and teach others by communication. Henceforth a social tradition comes into play, the past history of society acquires a significance, and action may be guided by a conception of the social future. Lastly, on the practical side these larger interests appeal to the self as a whole and often conflict with the solicitation of some more special and immediate end. In that case they prevail only when they can obtain a response from 
some dominating central impulse of the self wherein the desires are either harmonised or controlled. This central impulse is what we call the Will, and it is influenced by the relatively persistent feeling-tone of the self as desire is influenced by the temporary feeling attending its realisation. This relatively persistent feeling-tone is the backbone of Happiness or Unhappiness, or at least of internal Peace or internal Discontent.

Thus along with the concept and the processes of explicit reasoning which centre upon the concept there emerges the knowledge of self, and of other beings as persons, the formation of a social tradition, and that organisation of impulse that we know as will. Any one of these involves the rest and is distinctive of the human as opposed to the animal grade of development.

The world as conceived under these influences soon begins to be a very different world from that which is perceived. It is a world not of colours and tones and feelings, but of persons and things, groups and classes, quantities, qualities and relations, the stable fabric prolonged indefinitely into past and future, whose states, phases, attributes, changes make up the world of perception. To the higher development of mind there corresponds a deeper stratum of reality. As at the stage of Assimilation, Reality may be conceived as presenting itself in the form of sense-stimuli charged with feeling, and as at the stage of perceptual correlation it appears as a network of related objects of perception underlying and in a measure explaining the stimuli and their attendant feeling, so now it appears as a world of permanence in the midst of change, of uniformity shot through variety which is again to explain the perceptual order. Each advance of intelligence may be taken, on the one hand, as extending our grasp on experience, and consequently our power to direct life, on the other as yielding deeper insight into new orders of reality. The building up of the conceptual order however is a long and gradual process. It is essentially an achievement of the social mind, and the stages of its formation are in a measure recoverable from the examination of the actual movement of human thought. We shall find at least 
enough material to indicate some of the leading phases in the evolution, and we shall review this material in the next chapters. We shall there see clearly enough that the 'selfconsciousness' of the human mind is by no means the same thing as self-criticism. In all its earlier phases the operations of the mind, however clearly it be aware of its immediate object, are set in a framework which is built up by the joint action of social tradition and the hereditary structure of mind. When criticism is turned upon this framework a higher stage is reached.

The phase at present before us then may be characterised in general terms as that of the correlation of the common elements-universals-which run through the perceptual order. It arises as these universals, which previously operate unconsciously, emerge into explicit objects of consciousness. With their aid it arranges masses of experience in ordered groupings and forms general rules for the guidance of action. If, as before, we conceive it to advance upon its own plane without leaving it, if we put together all that may be effected by consciousness without necessarily constituting a unitary object of consciousness, we may say that it will effect a comprehensive order co-ordinating the general relations found in experience with permanent elements of well-being, and the experience would be social as well as personal experience, and the well-being social as well as personal well-being. But still, upon this plane, however far-reaching the order may be, the methods of correlation are determined by massive forces reaching far into the background of social tradition and racial heredity. When the work of correlation has advanced to a certain point the existence of these forces emerges into consciousness and provokes questions which give a new direction to effort. This effort initiates a higher phase of mental activity. The characteristic work of the stage now described is a Correlation of Universals based on the conditions of racial and social development which are not yet brought into consciousness.

(6) Correlation of Governing Principles.

The unifying work of thought has no assignable limit, 
and, in spite of what has just been said, it might seem that on the lines just indicated it could advance indefinitely towards a comprehensive view of experience and even of reality as a whole. But in point of fact the work of thought in the stage hitherto considered is broken and incomplete. Experience is gathered up into masses presenting some internal order, but not yet harmonised one with another. Our common knowledge is broken knowledge and half knowledge, a series of glimpses with no complete vision. In close correspondence our purposes are fragmentary and inconsistent, and we war both with ourselves and with one another. The roots of this discord can be traced to the conditions of development, and in following the movement of human thought we shall see how inevitably they result from the uncritical reactions of the social mind-structure to the promptings of its experience. Conversely, the method of advance towards genuine unity lies alike on the side of knowledge and of action through a process of reconstruction. This reconstruction, the general character of which will be discussed in Chapters VIII. and IX., will be found to depend, once again, on a fresh turn of the mind by which that which has hitherto operated on consciousness becomes a content in consciousness. In this case the factor in question is nothing less than the correlating activity itself, the structure of the mind, the entirety of the data and the processes by which and out of which the mind evolves its percepts, its thoughts and its purposes. The nature, the growth, the potentialities of mind itself form the keystone of the complete synthesis at which reconstruction aims.

And as at former stages so here, the new turn of thought brings us into contact with a deeper stratum of reality. As we passed from a 'world' of sensory stimulus and feeling to one of related objects and events, and from the network of percepts to the elements of common character and persistent identity running through it, so now in the critical reconstruction of knowledge we are dealing throughout with a new view of reality - the underlying forces, be they spiritual or mechanical, which are grasped indeed by means of perception and thought, but only when 
perceptions and thoughts are critically compared and systematically interrelated.

The distinguishing feature of this stage is the explicit recognition of the conditions operating on or in the mind itself, the entrance among the data to be correlated of the correlating processes or activities. It is a self-conscious correlation, a correlation of methods and result, or, briefly, of ultimate principles. If, once again, we conceive this critical movement carried through it would analyse our mental world down to its elements, and our purposes to the ultimate sources of their value, and it would bring them together into a working whole of rational comprehension and purposive activity. It would correlate the system of racial experience with the ultimate ends of racial development. It would thus cover the entire sphere of human life, bringing its past and future within the compass of a single synthesis. This would, in the first place, be effected by consciousness piecemeal, but in its completeness it would also come within the scope of reference of consciousness. The development of mind would come within the knowledge of mind, and it may be inferred in some degree within the control of mind.

With regard to the measure of this control more will have to be said. But we have first to justify our summary account of the two last phases here distinguished. This will be the task of the remaining chapters of this part. We have to begin with the building up of a partial and uncritical order of thought by the social mind. This will occupy the following chapter. We have then to deal with the work of reconstruction, which in its various phases will occupy Chapter VII. to X. Here we have briefly indicated the nature of these phases in order to take a summary view of the movement of mind as a whole. The latest phase described completes, it will be seen, that process of correlation which we have traced from its beginnings by bringing within the circle of consciousness all the factors that work upon consciousness. Throughout the development there has been stage by stage a change of orientation, engendered by the entry into the field of conscious intelligence of something that before affected the mind without 
entering in as a content, determined correlation without being itself a term in correlation. This series of changes would seem to be completed in idea by the inclusion of the conditions on which its own existence depends. We are now, therefore, in a position to take a summary view of the succession of phases in the evolution of Mind.

We have been led then to conceive of life as the process of a psycho-physical structure which grows up in interaction with the environment and which acquires, through the medium of correlations of which consciousness is the essential organ, the power of directing its own fortunes. In the lowest stages consciousness is undeveloped, and the response to the environment, at first wholly random and useless, is gradually directed in paths which are normally suitable to vital needs by the action of heredity. This is the stage of the 'Structural' action and the hereditary reflex, which controls the immediate response to direct sense-stimuli. So far correlation is based on the hereditary structure. To meet the individual variation of such stimuli a special correlation of co-present sense-data is required, and this is the first work of consciousness in sensori-motor action. The feeling-tone determining this correlation from moment to moment may be so adjusted, through the influence of heredity, as to guide trains of action towards ends of vital service. Such a train of action is called an Instinct. Correlation based on heredity is here combined with correlation based on present conditions. From this we pass to correlation based on Individual and Social experience. This yields first the formation of habit and trained skill resting on the correlation of sense-stimulus with feeling under the influence of related consequences. There is a massive, indirect or inarticulate correlation of individual experiences. In the next stages these consequences come into consciousness, distinct elements are grasped in their relations and anticipatory ideas are formed on the basis of perception under the influence of underlying affinities. Particular experiences are articulately or directly correlated. Next, these affinities come into consciousness and we have a Correlation of Universals,-in which experience is organised into bodies of thought and 
action subordinated to wide and permanent ends, while both the experience and the purpose are not merely personal but also social. Lastly, the deficiencies and contradictions of the thought-order force on a process of reconstruction by which the underlying factors of heredity, of personal experience, and of social growth which go to the building of consciousness, are themselves brought within consciousness. There is a correlation of results with processes or principles. With the aid of these principles it becomes possible to take a comprehensive survey of human development, tracing our life backward to its ultimate condition, and carrying its aims and efforts forwards to their ultimate meaning and goal, to correlate human purpose as a whole with the conditions of development as a whole. 


\section{CHAPTER VI}

\section{THE EMPIRICAL ORDER}

In the development summarised in the last chapter the two final stages were treated as the work not of one mind but of many. From the dawn of language onwards the action of mind on mind is the leading factor in development, and henceforward every phase of thought may be regarded as a social product and as a cause of further social effects. Our next task is to describe these latter stages in some further detail, to examine the steps by which in human society the thought-order is evolved, criticised and reconstructed. As before we shall find that every phase has its distinct method and its peculiar scope. It brings us into contact with a new stratum of reality in virtue of a new method of correlating experience, and it enlarges and clarifies human purposes in the same ratio. Our object then will be to distinguish the main phases of development experienced by the social mind in point of the characteristic methods used, and the scope of thought and purpose achieved. We shall find that particularly in the later stages a third question arises, that of the ultimate validity of the processes employed and the results attained. This question carries us outside our immediate task of recording the simple facts of the development of thought, but we shall find it so closely interwoven with the questions of scope and method that it will be impossible to eliminate it from the discussion. We shall, moreover, as explained in Chapter I., have to form a definite conclusion upon questions of validity in order to a just interpretation of the meaning and trend of development as a whole. 
The movement has many aspects and, though at bottom a unity, its essence can only be intelligibly explained by following each aspect separately. We shall deal with it first on the side of thought or cognition as such, then on the side of purpose, and lastly on the side of those social relations in which thought and purpose may be said to be embodied. In each case we shall follow the process as a whole from the evolution of general ideas to their critical reconstruction.

\section{(I) The Empirical Order.}

We have taken language as the distinctive mark of human intelligence because it reflects the conceptions by which empirical data are brought into relation. It not only reflects them, it is the condition of their effective use. Resemblances of quality are expressed by general terms, continuity of existence by individual names, the relation of ideas and the order of connection in thought by the arrangement of words in the sentence. As the work of correlation is social it cannot proceed effectively unless by means of expression, and the expression which is in the first instance an effect thus becomes in substance a most important determining condition of the further development of thought. Language and its early accompaniment, gesture, forms along with art the two principal vehicles of expression, and if we had a complete record of language and of art, we might reconstruct with fair completeness the earlier stages of the growth of the human mind. This, as the evidence stands, we cannot do, but we are able to distinguish certain phases of growth sufficiently to see that the general ideas which form our ordinary mental furniture have a history, that the process of forming them is one that only came to maturity by degrees, and that it reaches maturity only to give occasion for the higher processes of 'Reconstruction.'

Let us begin by considering the character of the process as a whole. As the work of correlation advances a certain order emerges within the chaos of perception. This order does not in its earlier stages amount to a system, still less is its formation guided by conscious and deliberate method. 
We are to conceive the great forces of the human mind as still operating from the background in the dark. The light of consciousness falls, as it were, on the surface of a deep sea of energy. It stirs forces that reach far down into the depths, and these forces determine the movements and rearrangements within the lighted area, but without themselves emerging into the light. Ideas are formed, names are given, judgments are passed, inferences drawn, emotions stirred, desires conceived and plans of action resolved upon, and the whole play is played out on the illuminated area. Perception gives the cue, deliberate action supervenes, and further perceptible results follow, but to trace the real causes to their roots we should have to go below to forces which are not formulated and are perhaps but obscurely felt. Nevertheless as the work of correlation advances, certain governing modes of conception begin to stand out. Without being recognised as explicit principles of correlation, possibly without even being named and known on their own account, there emerge certain structural forms of great generality which come to govern the work of correlation, give shape to the entire order, and direct the work of construction. These structural forms are what are known as the categories of common sense, such categories as those of substance and attribute, cause and effect, space and time, action and passivity, persistence and change, sameness and difference. Themselves educed from experience they react powerfully-and that long before they are named and known for what they are-on the empirical order. To understand this action it is not necessary to suppose, after the fashion of the Transcendental Analytic, that nothing could be experienced that does not conform to certain pre-existing categories. It is sufficient that whatever when experienced is seen to fall within the limits of one or other of them acquires thereby a certain local habitation within the existing order. What will not square with them is vague, meaningless and obscure. It hovers doubtful on the confines of consciousness. It cannot get itself expressed, nor enter into the ever-living medium of language, which alone confers permanence on the fleeting experiences of man, and so it flutters away 
again from the ordered world into the darkness of the inane. The victory of the categories is not established without a struggle, and like other victories it ends in a dictatorship under which death or exile is the penalty of recalcitrance.

The empirical order thus established on the solid foundation of the categories constitutes what we know as the world of common sense. The term empirical must not be taken to mean that the order consists simply of the series of sensations, emotions, or, generally, of the contents of immediate consciousness. Precisely because it is an order it is more, and also less, than these. It is the world built up out of these by unreflective processes of thought, imagination, feeling, action. It is the world of which men can give some account to themselves and one another. There is in it something of system, for the general ideas which it forms and employs serve to connect experiences and to direct actions. But it is an unsystematic system, for the principles of connection are never sought out beyond the point to which practical needs or casual interest may point, while the processes involved in establishing the order, though processes of thought are, as has been said, unreflective processes, that is to say their nature and implications are not examined. Nevertheless, though its conceptions are loose, its generalisations somewhat slippery and its methods uncritical, common sense does by slow degrees evolve a kind of order. We may even say that without deserting its own plane it evolves a generalised conception of order - the natural course of things-Nature, human, non-human, animate and inanimate as we know it in experience, and this order in fact governs our ordinary workaday life. It is this conception of nature and the loosely woven tissue of rules, ideas, views and practices that range themselves below it that I speak of here as the Empirical Order.

(2) The formation of this Empirical Order forms the first phase in the development of human thought. Its critical reconstruction occupies the second phase-the two corresponding to the two highest phases in the general evolution of mind sketched in the last chapter. But both 
movements break up into subordinate steps, which we proceed to distinguish.

The lower phase that precedes the consolidation of a common sense order is best understood by reviewing briefly the character of the looser and lower modes of the exercise of intelligence in the developed man. For the more primitive ways are never laid aside. They are merely overlaid and held in check by the more developed thought which is the distinctive product of the social mind at its best. If we suppose this influence withdrawn, we obtain some measure of the untutored mind of the child and of primitive man, and we can in fact corroborate our deductions by the direct examination of ideas and methods current in the lower culture. It will be sufficient here to distinguish two points in which the lower order of thought falls short of the methods and achievement of common sense.

(a) Common sense uses, in the organisation of experience, general ideas-man, animal, custom, good, evil, round, square, single, plural, which are in the main clear and distinct without being rigorously defined or systematically compared. They serve their own purpose, which is that of colligating experience, grouping together things which belong together, and focussing results for the guidance of practice. For these purposes the rough-hewn idea serves its turn. The 'round' is not Euclid's circle. It is a wheel which turns 'true' enough to make the cart go. The 'just' man is not one who conforms to an abstract ideal of fair dealing, but he is one whom his neighbours trust. Ideas at this stage serve to focus masses of experience, but are not themselves so clean cut and defined as to be capable of being built into a regular system. Where such systematisation of accurate thought-elements begins we are passing beyond the sphere of common sense into that of science. The common-sense concept is made what it is by rough and ready working of experience, which forces comparisons and distinctions, and so engenders enough of accuracy for many practical purposes, but not enough for systematic reasoning. We may call the concept in this stage a general idea. At the full height of their develop- 
ment such ideas though not defined are clear, and except for a certain raggedness about the edges, distinct from one another. Each embodies and expresses a certain mass of experience and that only. The achievement of such ideas represents a distinct onward stage in human thought, for one of the first points that we discern in the lower strata of the mind is the relative absence of such distinctness. But we must carry the matter a little further. Obscurity and clarity are relative terms. Throughout the history of thought new distinctions are constantly being drawn, and what appeared clear and definite is shown to have concealed ambiguities and obscurities. Thought does not rise out of the mists once and for all. What then are the kind of obscurities that beset childish and primitive thought? The answer may be found by returning to the categories of common sense. For common sense this is a world of substantial things possessing attributes, entering into relations with one another, acting causally on one another so as to produce changes. which are events occurring in time and space, and so forth. Common sense does not qualify these varying aspects of reality in abstract terms. When it does so it begins to be metaphysics or science, but its concepts do follow the lines of distinction prescribed by these several sides or aspects of experience, and in its maturity it does not confuse one sort of concept with another. Its substances are substances and its relations are relations. The characteristic of earlier formless thought is that it does make confusions of this kind and in particular it confounds the category of substance with the others. Thus the vital functions of men, animals or plants become a quasi-material essence, identified perhaps with the shadow, perhaps with the breath, capable of being caught, confined and transferred. A word or a thought may be a living force, and if charged with emotion like a curse, may be washed off a person or purged out of him. A pain is a stone that may be extracted, a quality like courage or timidity is an entity that may be transferred. In some of these cases we may say that a quality is hypostatised into something resembling substance, in others that a function or relation is treated like an inherent quality belonging to the sub- 
stance of the thing. The latter case would be well illustrated by the common savage notion of obtaining a man's courage by eating him or those portions of him in which the courage is held to reside. Even good fortune is perhaps a something that behaves like a physical quality, and at a higher stage grace may be transferred by the laying on of hands. Indeed the tendency to turn qualities, functions and relations into substances is very persistent at much higher stages of thought, since it arises from the difficulty of forming a clear concept of anything without conceiving it thereby as distinct and separate in its essence from other things, and what is so distinct and separate readily becomes self-subsisting. But if in early thought, relations and qualities tend to become substances, it is equally true that substances deliquesce into a series of changes. Transformations are effected with the greatest ease. The genie becomes a dragon, a seed, a fire. The big Bear that is in the sky is also incarnated in the bears that are hunted here. The soul goes far away, yet is affected by the fortunes of the body. What belonged to the body but is severed from it affects its fortunes as if it were part of the body still. By a quite similar order of confusion the general is identified with the particular. The ceremonial treatment of an individual animal serves as a bond between the whole species and the performers. When the totem is eaten a link is established with the class of objects to which the totem belongs, and to explain the character of a species a story is told of something that happened to an individual member of it. What is similar functions as though it were the same, so that the maltreatment of an image destroys the original, and to represent the fertilising process assists fertility. Indeed, whatever is connected with a thing in any way may retain strands of connection with the thing, so that shorn hair or nail clippings falling into the hands of an enemy give him physical powers over the original owner, and the sword that has made a wound will afterwards inflame it if allowed to rust, and should be kept clean and bright if the wound is to heal healthily. I call this mass of confusions which underlie the bulk of animism and magic - the two characteristic constructions of primi- 
tive thought-a confusion of categories, not as meaning that the categories having been formed are then confounded, but as meaning that they are not-so far as these constructions prevail - adequately distinguished and firmly established. Aspects of the empirical order which for common sense are clearly distinct remained blended and blurred so that we pass from one order of ideas to another without any sense of discontinuity. No lines of demarcation are fixed.

(b) But secondly, these confusions have behind them a distinct driving force which accounts for their extravagant development and persistence in certain directions. Common sense, though not ruled by conscious logic, is moved by its own determining forces in a broad sense along the lines which logic afterwards formulates. That is to say, it is guided by experience which it generalises with a certain caution, correcting and limiting one rough generalisation by another, and piecing the results of experience together by a rude analysis and synthesis. In the court of common sense, though there be no formulae, good evidence is already distinguished from bad, and good evidence consists either in reasoning from admitted data or in some fact or facts of perception to which one can point. Now to have come thus far in the course of rationalisation is to have advanced a stage in human thought, for we find below it a stage marked with tolerable clearness in which it is neither perception of relevant facts nor dispassionate reasoning from admitted data, but partly the drift of fancy and much more the sway of impulse-feeling which determines belief. By the drift of fancy I mean the incalculable movements of ideas in the imagination under the stress of chance associations, of the play of words and of other forces having no relation to the real evidence for a belief. By the sway of impulse-feeling I mean that in the lowest stages of the human mind ideas, propounded by no matter what, tend to be accepted if they suit our feelings, and to be rejected if they annoy. Acceptance and rejection are the primary attitudes out of which reluctant affirmation or denial are developed by differentiation. Ideas arise, as we have seen, in the practical sphere as the directive element in desire. 
Hence at the outset between the willing retention of an idea and the desire of the object to which the idea refers there is very little difference. Hence, further, untutored minds retain a difficulty in affirming resolutely that which they dislike to believe, and indeed this difficulty, like others that belong to the more elementary stages, persists in the highest thought, and not seldom influences it. In the lower thought it produces a regular make-belief, which clearly plays a large part in magic and animism as it does in the doll cult of children. The child likes to give the Teddy Bear a bit of its cake and to think that it eats it, but to make the eating real the child will cheerfully carry. out that process himself. The difference is not great between this play and the ceremonial in which the human worshippers eat the material sacrifice while the gods feed upon the spirit. As long as it is a source of mental comfort to think that a spirit has accepted a sacrifice and will be appeased by it the belief itself will flourish, demanding no more sustenance than the formal acts required by tradition with some sense of mystery, some unknown formulae or secret rites at the back, to draw as it were a veil behind which the transaction which the senses cannot witness may be supposed to go forward. Lastly, if the ceremonial is so arranged as to satisfy the motor impulses, if for the satisfaction of anger there is some smiting of a victim to be done, if in rejoicing there is dancing, or to summon the war god music and beating of drums, the action still more directly satisfies a felt want, and has a physical as well as a psychic appropriateness.

Indeed, in interpreting primitive belief it is possible that we ought very often to invert what is for us the logical order. We see food implements and possibly wives or slaves buried with the dead by some primitive folk, and we say 'They believe that the dead continue to live in much the same way and to need the same things: therefore they give them what they will need.' Perhaps what we should say is rather 'The mass of sentiments and emotions stirred by death impel the mourners to acts of respect, affection and sacrifice. As they come to give to themselves or perhaps to their enquiring children some account of these acts they 
can express their meaning only by speaking of the dead as continuing to live, so that the practice emerges from a sentiment, and in turn gives rise to the belief that would justify it.' If this explanation is correct neither magic nor animism is primitive. What is primitive here, as all through the earlier stages of psychology, is impulse-feeling, and here as in those earlier stages the idea formulates, directs, extends and in a way explains the act to which feeling prompts. This is at least one root of primitive belief. On the other hand the extensions of idea involved in magic and animism - the tendency to clothe one object with the attributes of another, not through conscious generalisation but through failure in distinction - seem equally ' natural' to the dawning intelligence. Idea may suggest impulse, or impulse may lead up to idea. Provided there is fundamental harmony with feeling, the ideas will be suited to their environment, and will survive. In general we may suppose that the magic ceremony, the animist's spell-prayer, the witches' charm all have an efficacy of their own-bringing relief to the tension of suffering or anxiety, or arousing terror and dismay in the mind of enemies at whom they are aimed. But this emotional suitability considered as evidence for truth stands materially below the rough logic of common sense.

We may then formulate the advance made in passing through the first two stages of human thought much as follows. With the origin of language there arises the germ of the power to group experiential data in accordance with their affinities, and so to build up conceptions of individuals, groups and classes as the subjects of rough and ready generalisations. With regard to matters standing out very plainly in experience or very close to practical interests there is not room for much divergence in method. People are not to be persuaded that thirst can be satisfied without drinking, or that a flint stone is soft to lie on. But outside the limited area of readily tested belief lies a mass of more doubtful ideas of great significance in human life. In this region we find in the first stage that the movements of fancy under the sway of feeling take the lead in forming belief, and that the ideas formed are so obscure and inconsistent as to blur the deepest lines of distinction drawn 
for more developed thought in the logical categories. We may then consider the first stage in human thought to be one in which the process of organising experience into the common categories is incomplete, and the evidence for the truth of an idea is not yet separate from the qualities which make it pleasant. The transition to the second stage, which we have called that of common sense, witnesses (I) the organisation of ideas in accordance with the categories, and (2) the differentiation of belief from feeling. Neither of these processes is to be understood as being derived as yet from any abstract principle. The categories are not known in the abstract, and there are no laws or formulae of truth, only experience has begun to shape the world of ideas and of language into that form in which logic finds it-the form in which concrete substances and their functions, attributes and relations are clear enough and are quite distinct from one another: the world again where proof is already one thing and liking another. These are the simple essentials of that empirical order which represents the workaday world as it presents itself to the average civilised man, outside the scientific laboratory, the church or the lecture room.

(3) Now this order and the methods which establish it are exposed to attack from more than one angle. On the one hand, there is the risk of self-criticism. This may be said to begin with the demand for exactitude, a demand to which practical interests cannot always close their ears. But the criterion of exactitude applied to loose generalisation means criticism and definition, and opens the road to science and philosophy, wherein the structural categories themselves will not escape attention. To this road we must return later. Let us notice first the other angle of attack. The world of common sense is not the whole world. Some would say it is not the real world at all. Whether that is so or not we shall have to enquire, but granted for the moment that its world is real, still it is not the whole world. Worse, it is not a world that explains itself. The forces that produce the play of action visible within it are not themselves within it. This is no metaphysical dogma, but, for us, provable fact. Take the course of a disease. Common sense moving on the plane 
of perception can diagnose certain symptoms, make a fair prognosis of the course of events, and apply palliatives or even remedies with a greater or less degree of practical success. The microscope reveals germs, and modifications of cell tissues, chemistry traces actions and reactions all invisible to common perception and all essential to a true understanding of the matter. Whether the 'scientific' account of causation is or can be adequate is a further question which for the moment we do not raise. Enough is known however to prove that even for the inanimate world the working of causation is definitely not discoverable on the plane of common sense. 'Man joins and disjoins bodies; the rest nature transacts within.' Thus was the measure of empirical knowledge and its practical application adequately and succinctly described. And if this limitation is true of the material order with its relative simplicity, still more is it true of life and consciousness. Man knows little of himself, but he knows enough to justify the broad truth of the metaphor used above, that the sphere of consciousness is but an illuminated spot on the surface of a deep sea.

But the forces within the depths are all the time at work. They direct our movements and give form to our thought. Nor are we in fact cut off from the knowledge of them by any impassable barrier like that which in some metaphysical systems separates appearance from reality. As the light gathers in intensity and concentration it penetrates here and there below the surface. But with regard to our knowledge of underlying forces we may usefully distinguish three phases. In the first place, to begin with that which is last in order of development but most intelligible in the order of logic, we might attain to a clear and untroubled vision of the forces as such. This would involve an enlargement of our experience as well as an improvement of our methods, of which we shall have to speak. In the terms of our metaphor it would imply that the light had penetrated below the surface to the depths. But in the second place and short of this we may have an obscure and imperfect glimpse of underlying realities. We may have a sense that they are there without knowing what they are, 
or (what is more frequent) our dim sense may clothe itself in a misleading shape of concrete definiteness, and we may feel an intense conviction of luminous truth when all the time the actual images that we possess are mere shadows, and what is working within us is something far other than that which we suppose. This leads us to the third and lowest grade, where the unseen forces are also unthought of, but where none the less they affect our thought, shape our theories and guide our impulses. In this capacity they are apt to intrude as disturbing influences on the world of common sense, arresting its work of systematisation on its own plane, by obtruding the element of the incalculable and bringing in the emotions of fear and hope to distort the cooler processes of inference and belief.

In this form underlying reality is at work upon consciousness from the first, and, as we have seen in the analysis of magic and animism, Thought even before the empirical order is formed is by no means content with the world that it can see and touch. It has its view of the processes that underlie the tangible and visible, and this view is in a certain sense a theory of causation and a conception of the supersensible. But in this connection we must be very careful to hold different stages of development apart. Neither magic nor animism is as yet in any strictness a theory of the supersensible or supernatural, because as long as they are dominant there is as yet no theory of the sensible and natural. More than that there is not in strictness any theory at all in the sense of a connected system of articulate thoughts. There are beliefs, ceremonies, practices, which we can reduce to principles and so form into a theory, but if those who held them possessed the same powers of reflection they would cease to hold them. Nor are the spirits of animism or the powers of magic supposed to be supernatural. Some spirits have mysterious powers. But spirits as such are just like ourselves, or they are the life or the functions of things precipitated into an image-

The troll and gnome and dwerg

And the gods of cliff and berg

Were about us and beneath us and above.

There was not one order of this perceptual world and 
another of the imperceptible. The magic rain-making was a quite natural process if anything was natural. The harvest might be improved as obviously by prescribed representations of a fertilising process as by the equally unintelligible virtues of manure. The distinction which makes the one method practical and relegates the other to the clouds of superstition exists for us, not for the honest magic-worker. No doubt some things were plain, while round them rose a thin cloud of mystery, which gradually deepened into an impenetrable veil, and no doubt it was within the cloud confines, and most of all in its darker recesses, that magic and spirit worship flourished most. But this is not to say that either magic or animism was an explicit theory of a deeper reality underlying the order which common sense had evolved. For magic and animism precede the formation of that order, while the contrast between experience and reality only comes into view after it is firmly established. The recognition of the supernatural as such is not primitive, but comes at a relatively high stage of development.

(4) But now if magic and animism belong in essentials to a lower stage of thought, what takes their place when the empirical order is formed? We are not to suppose that they are extirpated by common sense. On the contrary, they retain much of their power, but are overlaid by more developed conceptions. The mind is never satisfied with the empirical order which fails to solve many of its deepest and most urgent problems, and at every stage it meets the need with ideas of an order suited to and conditioned by its development at that stage. At the point at which the empirical order is well developed the animistic spirits are in greater or less degree subordinated to a god or gods who, like other objects of common sense, are clearly and vividly conceived. As compared with a spirit the god has a distinct personality. He has a home, on Mount Olympus or on Mount Seir. $\mathrm{He}$ has a history and a character, friends and enemies, very possibly wives and children. From an abstraction he has become something concrete. He has evolved into a man, and indeed into a superman, i.e. a being with human feelings but more than 
human strength. He is distinct from any material, from the stone or the image or the animal in which his ancestor the spirit was merged. Also in sympathy with the general extension of order in experience he has much more extensive powers than a spirit. From being the underlying vital principle of a tree he has become the god of vegetation, perhaps the god of all the earth or of the sea. Or again he is the god of the people, the centre of national patriotism, and destined accordingly to a higher elevation, to sit enthroned among the congregation of gods, to deny their right to worship and ultimately to existence.

Thus the divine takes independent shape, and the gods have a world of their own, a world on the border of the empirical, but neither threatening it with conquest nor divided by any very scientific frontier. Indeed, at the outset there is little difficulty in mutual accommodation. The empirical order is not so firmly established but that miraculous interventions may obtain credence, nor have the structural categories been thought out to the point at which philosophical difficulties interpose, nor has criticism turned its edge upon the foundations of the supernatural. There are rules of art, but the craft has a god to help with that divine touch which no rule can fully secure, to temper the iron to the right point, to raise the cream and keep the milk from turning sour. In the graver issues of life, where human control is still very weak, prayer and ceremonial are of wonderful psychic staying power, at lowest as an anodyne, at best as a tonic and an inspiration. The two orders help each other, and conflict is but occasional and unnecessary.

(5) It is otherwise as the organising work of common sense draws towards its limit. As it extends its sphere and begins definitely to conceive Nature as a whole, as a system,

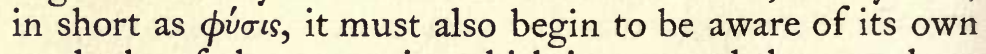
methods, of the categories which it uses and the postulates on which it rests. Long before this stage is reached the fatal demand for exactitude has been raised. The sciences of number and of space have begun to take shape, and accuracy has been practised in the records of astronomy. Alongside the looser ideas of common sense, bodies of 
accurately defined and nicely correlated concepts have arisen in arithmetic and geometry. Men have learnt what it is to observe and describe accurately, and the distinction between a vague generality and a strictly universal relation can no longer be missed. On this side the growth of science engenders discontent with the empirical order as rendered by common sense. On another side, it threatens the supernatural, with the demand for evidence and for consistency. The old easy-going acceptance of tradition is disturbed. The gods must give an account of themselves or vanish. With the consciousness of methods, postulates and conditions of sound thinking, we have passed the limits of unreflective development and entered those of methodical construction. Let us carefully consider the position at this stage, and the problem to be solved.

We first note the characteristics of the empirical order. To begin with, as its name indicates, it has been built up on the basis of experience, and the units of experience are objects of perception. Without seeking for the moment to analyse the phrase, we may point out that perception bears, not only upon the events of the material world, the qualities, motions and changes of material objects, but also on the inner world of consciousness, and that by the analysis and synthesis of perceptual data, by generalisation and deduction, we arrive at the connective ideas which we have treated as the essential tissue of the world of common sense, and which embody and connect for us, knowledge both of nature in the narrower sense of that term, and of human nature. Given that we can observe, and by analysis, synthesis and generalisation construct and apply ideas and judgments dealing with our surroundings, we have the simple foundations of common sense knowledge. When experience is specified as the foundation of common sense, it means experience worked up into an order of ideas by the factors specified. The same factors suffice to explain the power of calculation where even within the world of common sense we seem to reason a priori rather than empirically. For both the number and the space concepts are derivable from the empirical order by analysis, 
and calculation is at bottom a putting together, a piecing and re-piecing of the thought-elements so provided. Lastly, within the world of common sense and before we reach science, the process of inference is at least so far explicit that the distinction of grounded truth on the one hand, and fallacy, fancy, and make-believe upon the other, is fully apparent. It is here in particular that common sense represents an advance on the modes of thought typified by primitive magic, and offers a point of possible resistance to the dreamier world of mysticism and even of religion in general.

Now this common-sense method with its dawning science of calculation gives men a certain power of dealing with their environment. But it does not meet the fundamental problems of life. It gives men neither practical aid nor mental peace in face of the issues of death, of disgrace, of the deeper moral difficulties, the more searching problems of social life. The reason of its failure has been set forth already. It is that though it moves with some sureness within its own area, its area is, relatively speaking, the surface of life, and there are depths below the surface in which the springs of life lie hid. We have seen how from these springs arise the impulses and sentiments that get themselves clothed with ideas and embodied in traditions. As long as common sense is itself only struggling for existence, tradition passes unquestioned, and the gods survive. But as soon as the empirical method gains the confidence that comes from success on its own lines, a new position is reached. The adult mind will make a corresponding demand on the religious tradition. Men will by no means be contented to leave fundamentals alone, but in dealing with them they will require a certain logic, a certain coherence, a certain account of the relations between the proposed solution and the empirical order in which, so far as it goes, they have come to place deserved confidence. In short, as there has arisen a natural or practical order so there must now be a reasoned religious ordera coherent theory of final problems, and between the two orders there must be an intelligible relation. These requirements set the problem to the higher religions and the 
philosophies of the world. We may broadly sum up the position in a couple of sentences. Slowly asserting itself against the illusions and confusions of primitive thought, common sense has laid down the lines of an empirical order in the world of perception. But this order renders no adequate account of the foundation problems. For their solution a theory of reality is required, and theories arise with a coherence and cogency of their own, but distinct from, and even opposed to the teachings of common sense. Thus there come to be two orders of thought, and the problem of their relation constitutes the task set to the higher stages of mental development. The work of the two lower stages is essentially to evolve the empirical order, that of the higher stages is to relate the empirical order to the underlying conditions of reality. 


\section{CHAPTER VII}

\section{THE TWO ORDERS}

(I) THE problem of thought in its higher phases, whether as Philosophy or as Science, as Religion or as Art, is one of Reconstruction. The origin and nature of this problem only becomes fully intelligible in the light of the theory of evolution. That the deliverances of ordinary perception and the inferences of common sense should possess a certain validity and yet provide a very inadequate basis for a final interpretation of reality is in general terms perfectly intelligible to the evolutionist. For the student of mental evolution, perception and thought are alike functions of a structure which has grown up under the conditions of survival. What is generally necessary to such structures is merely that they should answer their purpose, and their purpose-or rather their function-is that of preserving the stock. For this it is necessary at bottom that they should induce suitable motor responses to changes of the environment. One way in which this might be done is certainly by the growth of a structure whose function should consist precisely in cognition-in knowing what the environment is, how it changes, and how it is going to change. But (a) this is not the only possible method of adjustment. The study of reflex action and of instinct yields overwhelming evidence that behaviour may be adjusted to the requirements of the organism in accordance with changes of the environment without knowledge on the part of the organism of what it is doing or why it is doing it. It is thus at least possible that there should be a point to point correspondence between changes in the 
environment and changes in the organism resulting in behaviour suited to the needs of the organism, yet nowhere implying knowledge of what is going forward. So theoretically it is possible that there should be a point to point correspondence between our thought, or any portion of our thought, and the real order without a true apprehension of that order. Indeed, if we take the scientific order as real, we are directly forced to admit the existence of such a correspondence at the lower stage of common sense, wherever we arrive at sound practical conclusions by methods or on grounds which are inadequate or false. The familiar experience of day and night and the observed position of the westering sun suffice to tell the savage that the darkness is at hand, and he will take his measures accordingly, and not a whit the worse because his mental construction of the sun's movements is scientifically false. The housewife can boil the kettle though she is innocent as the babe of the thermal laws involved in the operation. True, there are occasions on which the limitations of common sense will come into play. It is not prepared for all the exceptions which science can understand and foresee, and here the difference between a deeper and more superficial knowledge will break out and have practical consequences. Both in its successes and in its failures, the structure of common-sense knowledge reveals itself as a development adapted to the normal course of human environment, and adapted primarily to action within that environment and to understanding only as a means to action. The circumstance, then, that common sense has its validity as a practical guide is not to be taken, without further parley, as evidence that it renders a true account of our surroundings. It neither excludes this possibility nor decides in favour of it. We may reach sound practical conclusions from wrong theoretical premises.

(b) There is a further point of great importance. Even if our common-sense knowledge be sound as far as it goes, it may also be very inadequate. It is, to begin with, limited by perception. Now our perceptive faculties grow up under the ordinary conditions of development and they evolve - apart from artificial selection and training 
-only to the point of signalling to us certain changes in the environment. Wherever in any species this signalling apparatus is adequate to the maintenance of the stock at a sufficient rate, no further development is to be expected from natural selection and the laws of inheritance. Hence in the lower orders of the organic kingdom where the rate of reproduction is very high, and it is possible for very large numbers to perish in immaturity without the destruction of the stock, a very low grade of accuracy in response may secure the survival of a sufficient fraction of those born to carry on the life of the species. As the rate of reproduction diminishes, the individual acquires a higher value, and the necessity for judicious action becomes more imperative. Greater powers of perception and inference are evolved, and the fine structures of eye and ear come into being. But these grow up by the increasing specialisation of structures that are originally rude, and limitation is written over every page of their history. Thus the ear is only susceptible to the impulse of aerial waves of a certain length and frequency. To other waves differing from these only in quantity it is deaf. The eye begins to respond to transverse waves of a certain length, and there arises in our consciousness the sensation of a dark red. As the wave lengths diminish, the colours change till they reach the violet and then again it is dark. We have no sense organ to respond to electrical waves as such. We can see nothing distinctly that does not subtend a certain definite angle upon the retina, and the optimist who told us that man had not

the microscopic eye

For the plain reason-man is not a fly,

wrote before the days of bacteriology. Could man by direct perception have seen the microbe in the infected substance, the history of medicine would have been very different. In place of this means of combating disease, man has only some indirect and very imperfect perceptual signals - the disgust at putrefying substance, the aversion to the spectacle of disease, the fear of infected persons, the early preference for cooked food, the aversion to close 
animal contacts and so forth. If we ask why man is left without a power so useful, the answer can only be given in very general terms. Negatively, the human body is not the product of a finished design adapting it accurately to all its needs. Positively, it is the development in all its organs of a far ruder structure. By a ruthless elimination of failures, the organs are rough-hewn and finally polished down at certain points to an accurate adjustment to requirements. But nature makes no inventions like telephones or microscopes. It works upon what is there, and in perfecting specialises it to one function, abandoning others. If the human race can get along and survive with sight adapted to our colour scale and to the sizes and distances which we familiarly judge, that is enough for nature. The fact that man would do infinitely better if with this he could combine the eye of the telescope and the microscope is nothing to her. For to drop the too ready metaphor of personification, the physical structure is determined only by the conditions of survival, not by the requirements of an ideal type or a perfect economy.

It is not only in its data but in its use of them that ordinary thought betrays its origin. The common-sense concept is a practically-useful concept, and as long as it 'works,' common sense cares little for criticism. The 'solidity' of the table means that it will give you a nasty bump if you run against it. That is definition enough for the workaday world. The structural categories which appear fundamental and tend to be used as sieves which only let certain kinds of experience through into the admitted tradition, are in fact products of certain elementary processes, which have been specified, working within the empirical order. They are growths, and they have arisen at the outset under fundamentally the same conditions as those which we have traced in the rise of perception. Only as human purposes develop and truth becomes an object do more refined conditions come into play; of these conditions, and of their growth in general, we shall have a word more to say at a later stage. For the moment we may be content to note that whether we look at its data or its methods, the whole structure of the 
empirical order reveals itself as a specific development arising under conditions which show that it can be at best only a partial rendering of certain aspects of reality.

As this rendering is found inadequate, as deeper experiences and larger needs take shape, the second order is formed, and we get the dualism of religion and common experience. In this dualism the relativity of common sense is insisted on, and the need for an absolute truth proclaimed. But the truth which claims to be most secure rests on methods which are most fragile. It is indeed itself like common sense, a structure determined at bottom by the response of the Mind to the conditions under which it lives and grows, and though critical in that it demands internal order and coherence, it passes without that selfexamination which would reveal the fundamental insecurity of the whole fabric.

(2) In this account it will not have escaped the critic that we are assuming a good deal. Virtually, we have been speaking as though reality in some of its main features were known to us. We have been assuming that there is a certain environment, material or otherwise ; that living, conscious organisms arise within this environment; that they respond to its changes and thereby preserve themselves and are able to produce and bring up their young; that in this way, in accordance with the ordinary view of heredity, new species are formed, organs develop and so forth. Assuming all this we can in a general way understand how the ordinary conception of the empirical order might arise, and how it might have a certain practical validity and yet be a very imperfect, possibly a wholly false rendering of reality. But what guarantee have we for any one of these assumptions? How do we know anything about this reality which is distinct from the empirical order? The empirical order is that in which we ourselves live, in which our thought moves and has its being. How are we to get beyond it? The reply is that in one sense we never get beyond. Our own experience and our own thought remain the sole basis of our knowledge. If they 
yield us no truth then we possess none. Nevertheless experience as extended by observation and experiment, as refined and remodelled by analytical and comparative methods, undergoes a reconstruction which it is logically possible to take as genuine knowledge of reality, while recognising a narrower experience and a cruder thoughtorder as an imperfect and even misleading interpretation of the Real. There are here assumptions as to the final validity of thought which require justification, and the lines of this justification will be summarily indicated at a later stage. Our first task will be to follow out the process of reconstruction itself in its principal steps.

(3) Viewed very broadly, the work of reconstruction may be said to fall into two main phases. In the earlier, the mind works with the ideas that it has educed from experience. By meditation, by analysis, by interrelation, it seeks to transform them from loose generalities into exact concepts, to elaborate a systematic order, to achieve internal consistency. It feels that truth is one and consistent in all its parts, and if it could arrive at a wholly consistent body of thought, it would be confident that it had attained truth. It thus effects a Conceptual Reconstruction, yielding a view of Reality which differs widely from that of common sense. It does not necessarily neglect experience, but in truth, until the conceptual order is well developed, it lacks the necessary instrument for advancing the investigation of experience materially beyond the point which common sense has already reached. The first phase then is preparatory to a second in which the critical investigation of experience itself is the dominant factor, in which the foundations of the older conceptual order are dug out and laid anew, and a more fundamental reconstruction is begun. Of these two phases, the earlier was the special work of the ancient, the latter of modern thought. But we are not to infer that the work of antiquity was done once for all, and that the modern had simply to begin where the ancients left off. On the contrary, the problems raised in the early stages of reflection are still unsolved, and only open deeper issues as they are 
further examined. Nor are old methods superseded, but at best enlarged and supplemented.

As there are two phases in this development, so there are two great impelling motives persisting throughout and leading to very different forms of reconstruction. We saw that the common-sense order not only failed on the side of knowledge, but even more conspicuously in the matter of the spiritual necessities of man. The craving for spiritual satisfaction is as potent an influence, at least in the earlier stage of reconstruction, as the desire for consistency, completeness or, to put it generally, for truth for its own sake, and we may broadly distinguish attempts at reconstruction in accordance with the dominant motive as primarily religious or primarily scientific and philosophical. But we must not hastily assume any simple order of succession as between the two. On the contrary, the religious and the philosophical movements influence, and even interpenetrate one another. Nor can we here endeavour to trace the filiation of thought, for which indeed many links are wanting. Just this much may be said. In the oldest civilisations of Babylonia and Egypt, the early invention of writing first made possible a connected development of thought from generation to generation. The formative sciences began to appear towards the close of the third millennium в.c. We have an Egyptian text-book on arithmetic with some matter of algebraical character, and some geometry-not pure land-measurement-from the I 8 th dynasty, pointing back to sources as old as the I 2 th dynasty. We have early Babylonian tables of squares and cubes, and we have the early observational astronomy. That is to say, we have the beginnings of an orderly and systematic treatment of certain subjects. Further, through the second millennium we have clearly in Egypt a growing dissatisfaction with the traditional popular polytheism, and an effort towards a more coherent and spiritual conception, whether monotheistic or pantheistic in tendency. But it is not till the first millennium, perhaps from about 800 B.c., that we get a decided movement, and then during the next three or four centuries we find something that looks like a wave of higher impulse spreading over the centres of 
civilisation. We have the development of Brahmanism in India, the beginnings of ethical monotheism in Hebrew prophecy, the mysticism, and close upon it the ethical idealism of China, and finally the philosophic movement in Greece. What measure of interconnection we are to postulate among these movements, how far we are to suppose a direct propagation of ideas, or at least of stimulus by unknown contacts, how much is due to independent development, it is not yet possible to say. But during that period the foundations of our own thought and religion were laid. The thinkers of that time still speak to us. The questions they raised are still our questions. Of the creeds, systems, and methods of thought which have since dominated civilisation, Brahmanism, Buddhism, the Confucian ethics and Greek philosophy and science were born within that period, while Christianity and Islam were engendered later, out of the influences which then came to birth. This, then, is the foundation period of the Reconstruction.

(4) It is not within our purpose to follow the movement historically, but to distinguish its leading phases, noting only those points which have special significance for the general development of Mind. We must deal first with the work of the religious impulses, which in their dissatisfaction with the empirical order, urge the Mind on to the creation of a world of its own.

For it is the irony of human thought that experience itself forces on man problems which it cannot solve, and yet successively destroys all solutions which rest on any authority but its own. Not that religion is wholly divorced from experience. There are at the core of religious psychology elements of genuine experience, which as experience is just as real as the sensations of heat and cold. There is a true spiritual insight, that is to say, an apprehension of the workings of the psychical, a sense of those deeper realities on which our personal life and our relations to others rest. Such insight is for most men fitful, and reached only through some experience heavily charged with emotion. It may come in the romance of love or through the equally passionate and less selfish 
devotion to a child, through the stress of danger, or of temptation, or more calmly and equably in the communion with nature, or in the clear-sighted vision of large human issues and the ordered movement of the world. What we actually experience in such cases takes shape in our ideas and still more in the language in which we seek to describe it in accordance with the traditional religion of our time. If we could get the experience 'pure,' i.e. stripped of all the inferential implications which description involves, we should have a core of reality as sound and solid as our experience of space or of motion. But the case of religion is one in which it is more than usually difficult to get our experience 'pure' and unmixed with extraneous elements, and the movements of the religious consciousness are subjected throughout to the great driving force of the demand of feeling, in the widest sense of that term, for satisfaction. Man requires to be in some sort reconciled with his place in nature. He asks for consolation in grief, redemption from sin and disgrace, stimulus in practice, the guidance and encouragement of an ideal of character and a rule of life. For these emotional needs, bound up with much that is strong and good as well as with much that is weak and poor in our nature, he looks to religion for satisfaction. The religious doctrine that is to prevail must answer to these needs, and thus it will embody elements responding not only to our personal and selfcentred cravings, but to our ethical and social feelings and ideas, to our sense of justice and mercy, possibly also to our lust for battle, domination and cruelty. The ethics of an age or a people will be reflected in its religion, though, let us note, they will also be reflected back by its religion, modified in character, intensity or direction. The causation is not one-sided, but reciprocal, and so far as religion can take up a new demand, absorb it into its system and find a vent for it in some new form, it may survive change and preserve itself by adaptation. The plasticity of Christianity and more particularly of Romanism in this regard has been a main condition of its prolonged hold on vastly divergent masses of men. But this does not affect the main point. Religion cannot be imposed as a rigid 
system on any sort or condition of men without regard to their characteristics. There must be either an actual harmony or the conditions of a possible harmony which will grow if the religion is to be a vital part of the social structure. This necessity operates throughout the history of religion. We have seen it at work in the lowest stages of belief. But there it operated without check. As thought in its advance becomes clearer and more articulate a new condition of harmony appears. There must be intellectual or speculative coherence. The deliverances of the religious mind must consist with one another. There must emerge a dogmatic system forming a coherent and ordered whole. But order and coherence are of the essence of logic and reason, and religion must therefore make its account with these factors of mental life. Again, there is a parallel development of ethical feeling, which as it becomes conscious, demands a greater measure of harmony in personal and social life, and the religious system must provide a basis for such harmony and discard elements of teaching that conflict with it. The higher religion therefore sets up a definite and reasoned construction, a theory of the world and of man, an ideal of life, a unified system of thought and action.

But though there are logical and ethical conditions under which the religions move, they are not based squarely on experience, nor is their practical order educed from an investigation of the actual conditions of harmony. They take up a position above experience, and reasoning downward therefrom determine the destiny of man and prescribe the laws of conduct. Their appeal is in the last resort to 'faith,' to the inner light or to the wisdom of the illuminated. They may use historical narratives or miraculous signs as buttresses of faith, but at bottom they know that these are only outworks to impress the vulgar. The religious order stands on its own basis. But as the common-sense order is equally firm the result is a virtual recognition of two orders such as may be said roughly to express the attitude of popular Christianity. Here is our world, the world of space and time, of inanimate matter and of conscious human life, the scene of our personal 
history and the theatre of our efforts. Over there beyond the bounds of death is another world, where we shall live again and where the Kingdom of God is now. Both worlds are real, and for all practical purposes both have their own laws. Doubtless God rules this world too. He made it out of nothing, and could destroy it as a slip of paper in the fire, but it is part of his plan to let it run its course guided by the immutable laws of matter and the free will of man. Our guidance in this world is the empirical order as elaborated by science. Only on the side of ethico-religious duty do we come into regular contact with the spiritual order, and direct interventions of Providence in answer to prayer are irregular and uncertain. The two orders issue, in theory, from one being, but in practice they are two. They touch here and there and mechanically interact, but in the main they are self-dependent and equally real. Substantially, this form of solution may be regarded as the common property of Monotheism, the tendency of which is always to conceive of the Deity as Creator and Ruler set above and over, and so outside the world, which is accordingly a separate entity. That any such theory must make its account with the opposite drive towards Monism, which would merge the world in the Divine nature, is an interesting point. It is also the source of many logical and moral incoherences and inconsistencies which need not detain us here. It is sufficient to note the extent to which a distinctly dualistic system is possible, and to observe that it is stronger in popular practice than in the closer reasoning of theory.

(5) In view of the moral incoherence of the world of experience, the alternative to Dualism is to make the spiritual world the one reality, wherein the world of common-sense experience is either mere illusion or a passing and temporary phase. Such is in fact the tendency of the Brahmanic philosophy in its most thorough-going form. ${ }^{1}$ The real is One, and the Self is that One, and this

${ }^{1}$ Taking the Vedannta system as interpreted by Sankara as probably the most logical interpretation of the Upanishads (see Mr. George Thibaut's Introduction to the Vedanta Sutras-Sacred Books of the East, Vol. XXXIV. esp. pp. ciii. to cxxvii. 
self, smaller than a grain of mustard seed and wider than the heavens, passes through all the transformations that make up the life of the world. It lives in every man and in every insect. It does not come into being nor perish, but is the subject of an infinity of incarnations in the bodily prison. Or does it really change or suffer at all? By austerity, by self-repression, by knowledge, by retirement into the innermost recesses of the mind we may each of us find that self, and be at one with the central essence of things, and for him who is so at one and so at peace the husks of the body, the wrappings of sense fall away, the web of Mãya is brushed aside and the reality appears one and unchangeable. The world of space and time, the world of the finite individual, it would seem, is all delusion, and we are left to ask ourselves, is delusion itself something real, is error, though it contains no truth, something that truly exists and has a meaning and an importance for the life of the one?

The final tendency of spiritual Monism is clear, but it becomes clear only to show the insuperable difficulties that would flock about it if pushed to the bitter end, of which not the least violent is the practical one that life must be bent by the strongest, most violent efforts to the supreme work of negating and overcoming that flesh, that outer world which does not in reality exist, to conquering an illusion which in a world that is all Spirit has no intelligible source.

(6) The theoretical and moral paradoxes of a spiritual interpretation of the world-order may lead by reaction to mere scepticism which is the abandonment of any attempt at a consistent theory, or they may lead to a more cautious reconstruction of the spiritual order avowedly on the basis of practical needs and with an abandonment avowed or half avowed of the search for the ultimate truth. The second was the line of thought which in the East culminated in the great system of Buddhism. Here there is in a sense no theory of ultimate reality, for the world as known to us has no reality, at least no substantial reality. It is a world of Impermanence, of flux. Yet it is a world in which we may have to play our part, and our part is to 
disentangle ourselves from the delusions, the unreal desires, and the consequent sin and suffering involved in Selfhood. We are to escape now not by withdrawing into the recesses of the true Self, for the Self is no longer true or real, but rather by rising into a purer domain of perfectly selfless and impersonal love, which is to issue forth to all the quarters of the universe. In this emancipation of the Arahat there is true peace to be found for unhappy men within the circle of this life, and through it alone is the misery of individual life to be finally extinguished, since the attainment of true Arahatship puts an end to the Karma which would otherwise give rise to another vexed personal existence. The solution is practical rather than theoretic. It gives no ultimate account of the nature of things, but prescribes an order of life for man based on the practical and emotional needs of an outlook tinged with melancholy but softened by compassion. From our present point of view it may be regarded as a form of spiritual Monism, for, though it holds ultimate reality unknowable, still, for all practical purposes its spiritual order is real, and the only reality that counts. The layman, indeed, may accumulate merit and advance upon the Path without donning the yellow robe of the mendicant, yet it is not through success in the dealings of ordinary life that he will progress, but only by clearing his own mind of personal longings for anything that therein is.

It has already been remarked that the religions on which I have thus briefly touched are not altogether uncritical religions. They have, indeed, a history behind them, they have grown out of the uncritical folk-religions of an earlier time, and sometimes retain embarrassing traces of their past. But a profound religious experience, a wealth of spiritual insight and a great store of human and social feeling has gone to their making, while on the intellectual side their doctrines have been built up with the aid of all the resources of the subtlest dialectic. They do not, in fact, mature until thought in general has been refined to a stage at which an accurate logic and a subtle dialectic are the common property of the learned. They are moreover guided by the idea of unity in life and experience which is 
the focal point in the higher stages of the correlation of experience. On the other hand, the religious order remains self-poised, independent and even indifferent to ordinary experience. To the mystic mind, or in moments of religious elation, it may seem to transfuse that experience, but it does not really do so, and the reason is that any such transfusion must be a mutual process. There must be a movement from the empirical order itself before a true unity can be formed. Of this movement we shall have next to speak, but we may first endeavour to sum up and explain the results now reached.

We have passed beyond the rough and ready results of what we have called 'common sense' to a third, or systematic stage of human thought. By methods which have been briefly touched upon an empirical order has been formed, and its growth has ceased to be wholly unconscious. In varying degrees men are aware of its method and tendency. On all hands it is allowed by practice, if not in strict theory, a certain validity. But it is also clear that it does not exhaust reality, and in the opinion of many its value is quite secondary, and even at bottom deceptive. Side by side with it-theoretically, perhaps, in place of it - another order takes shape. This is in general what I have called a spiritual order, and it rests at bottom on the felt needs of man. But it no longer satisfies these needs by an easy acceptance of tradition. For it is also an order; it is developed with a regard at least for logical consistency and internal coherence. With varying degrees of completeness and success it seeks to satisfy the cravings of men. It propounds an ideal unity of thought, of character, of action, and thus offers a synthesis that is immeasurably wider, as its analysis probes far deeper, than the fragmentary judgments of common sense and the uncritical traditions of the folk-religions. But at the end the satisfaction that it yields is the main proof that it offers of its truth. Such a proof is not recognised in logic, and in methods as in result religion and experience fall asunder. There are two orders, and between these two men have, alike in theory and in practice, to effect a choice, a compromise, or a synthesis. 


\section{CHAPTER VIII}

\section{CONCEPTUAL RECONSTRUCTION}

I. If the common-sense order could not satisfy the spiritual cravings of man, neither could it, without serious modification, meet the demands of science.

Scientific thinking, indeed, is not distinguished from common sense by any peculiar assumptions, by any limitations of method or by any restriction to one field of experience rather than another. It is distinguished, first, by its motive. It aims at the discovery of truth and at no other result. It is detached from emotional, personal or practical objects. It is distinguished, secondly, by its continuity and exhaustiveness of treatment. It is not content with isolated results, but conceives its subject as a connected whole and investigates all that it can find which has a bearing thereon. It is distinguished, thirdly, by the exactness which in all its results it seeks to attain. Detachment, continuity and accuracy ${ }^{1}$ are the three marks of any science, and any study so marked is scientific, no matter what its subject may be.

Now continuous, consecutive and accurate investigation arises at an early stage in relation to the arts and handicrafts, and though the motive here is in the main practical, we have in the training of the craftsman the beginnings of system. In the early Oriental civilisations we have,

1 It is a part of accuracy to state definitely the degree of indefiniteness attaching to our knowledge and the measure of probability attaching to what is uncertain. Hence science is not limited to the definitely known, but also measures the degree of our knowledge where it is incomplete. 
further, the beginnings of genuine science. We have Egyptian text-books of arithmetic, and the elaborate astronomical records of Babylonia, while the practical requirements of land measurement laid the foundations of an empirical geometry. But with the early Greek philosophers a new epoch opens. ${ }^{1}$ The Ionic philosophers conceived the ideal of interrogating Nature without regard to tradition or to the requirements of the religious consciousness in the simple belief that they might find out her secret by reasoning from common observation. They attacked the problem of reality with simple-minded confidence. Modern research goes to show that their theories of the nature of things were crude but intelligible generalisations of experience as they interpreted it: on the question what reality is they agreed that it was something different from reality as it appears, yet whether they took Water, Air, Fire or the Flux of things as the ultimate reality, they founded themselves at bottom on facts of experience which they took to be fundamental and extended by simple and uncritical generalisation. But with the rise of the Eleatic school a new method appears. Reality according to the Eleatics must be one, not clearly because in experience we find that all things are one, but because the conception of Unity satisfies certain intellectual needs. Reality in general from this time forward becomes subject to the character and relations of the concepts by which we can interpret it, and there arises accordingly a systematic effort to construct reality by means of an examination of thought and its products. But the thought-product itself required criticism, and to supply a regular method of criticism was the work of Socrates. The Socratic dialectic aimed in the first place at the accurate definition of meanings, and proceeded by two methods which might be used separately or in combination. On the one hand, a concept might be examined by relation to the experience which it appeared to formulate. This was the foundation of a scientific induction. On the other hand, it might be tested and defined by comparison with other concepts. It would

${ }^{1}$ At least in Europe. I will not here enquire how far the conditions mentioned below are satisfied by Hindoo thought as well. 
then appear as a species within a genus, or as a genus containing species, or in both relations when the two points of view were combined. This was the foundation of the logic of deduction and of the Classificatory method of systematising knowledge. On these methods successive thinkers from Plato onwards built up theories of Reality with the character of which we are not here concerned. What is important for us is that in so doing they worked out the fundamental categories of experience, defining and distinguishing substance, attribute and relation, quantity and quality, the various forms of causation, the contrast of the universal and the particular, of the necessary and contingent. Nor could these distinctions be carried far without raising the problem of knowledge, the grounds of belief and the principles of reasoning. The world of reality, which is also that of the necessary, the universal and so the eternal, matches the system of accurate knowledge demonstrable by deduction from first principles, while the contingent, the changing, the indefinite, is the sphere of unscientific opinion. The method of demonstration is elaborately set out in the Aristotelian logic, and the relation of its first principles to experience is summarily indicated. They are educed by intelligence operating upon data of sense, but the logic of the operation remains shadowy.

The structure of thought in its main outlines was thus revealed by the great philosophers. But meanwhile another movement was on foot. As the problem of reality developed it soon became clear that it must be broken up. Mathematics and astronomy were making progress, and Plato distinguishes five special sciences, while Aristotle lays down a general theory of scientific specialisation and indicates the relation of science to metaphysics. Every science has its own particular field, and, in addition to the principles common to all reasoning, has its own specific principles, consisting in the primary definitions of its subject matter. A special science is conceived as a systematic body of truth educed by syllogistic reasoning from certain original definitions and axioms - the ideal which Euclid sought to realise in geometry. But, in fact, science 
was not purely deductive. Systematic observation was practised in astronomy, and by Aristotle himself in biology and sociology, while in the hands of Archimedes experiment guided by mathematical genius of the first order laid the foundations of mechanics. But the ancients had neither the mathematical methods nor the physical instruments which have given to experimental science the range which it has obtained in the modern world. Thus it comes about that when Greek thinkers move outside the region accessible to common observation, they give us conjectures rather than true hypotheses. These conjectures are often singularly brilliant and happy. The atomic theory of Democritus, the evolutionist suggestions from Empedocles onward, bear an interesting analogy to modern ideas. But it is easy to overrate their significance. Modern science, as will be remarked later, often obtains fruitful results by assuming positions which it cannot directly prove because it has worked out methods of reasoning from such assumptions and comparing its results with those of observation. An assumption so treated is a hypothesis. One which cannot be so treated remains a conjecture, and Greek theories of that which lay beyond the domain of direct observation remained for the most part conjectures. It needs no lengthy argument to show that it was in the construction of the conceptual order itself that the main work of the Greek enquirers lay. Thus we have on the one side the fundamental metaphysical enquiries, the analysis of the elementary categories, the statement of the philosophical problem, the elaboration of a deductive logic, the exposition of the ideal of knowledge and truth. On the other hand, we have the positive development of mathematics beginning along with the first philosophic impulse, but continuing long after philosophy had reached and passed its first culmination. We have the first completely systematic exposition of a body of truth in Euclid, the development of theoretical arithmetic, and, growing in importance at the close of Greek activity, the beginnings of algebra. Then we have mathematics applied to mechanics by Archimedes, and to astronomy by the long series of investigators whose work was ulti- 
mately reduced to system by Ptolemy. Not that observation was neglected. On the contrary, in astronomy results of great magnitude and, relatively to the instruments available, of surprising accuracy were attained. The catalogue of fixed stars made by Hipparchus was the best available till the time of Tycho Brahe. The same observer measured the length of the year within six minutes, discovered the precession of the equinoxes and knew the difference between the solar and the sidereal day. Indeed it may be said that after the banishment from Athens of Aristarchus for anticipating the Copernican theory the theoretical development of astronomy was small as compared with the advance in the description and accurate measurement of the phenomena. Thus it would be true to say that in astronomy the Greeks had laid the foundations of that union of mathematical reasoning with exact observation on which physical science depends. It would also be true to say that in biology their observations, again relatively to the available instruments, were searching and valuable. On the other hand, it is clear that on this side of knowledge in tracing the history of Greek enquiry we are dealing only with beginnings. If observation is rich in certain quarters of the field it is restricted to those quarters, and generally lacks instruments of precision. Direct experiment again is rare. Such an investigation as that by which Ptolemy determined the angle of the refraction of light in passing from air to water, etc., is quite an exceptional occurrence. Nor in spite of Archimedes at Syracuse, or of the more regular and continuous labours of Hero was the application of scientific principles a field of general interest to the Greek enquirers. In short, their body of recorded, organised, systematised experience was relatively small, and most of it moreover was gained in the later stages of their activity. It was in fact the natural outcome of that elaboration of the conceptual order which was the main work of their first and greatest period-an elaboration which laid the foundation alike of metaphysics, of ethics and of mathematics.

Now this work of conceptual construction is not a stage which thought has passed through and left behind. It is 
not a level which thought reached, say, in the age of Parmenides, and left for something higher at some point. in the development of modern philosophy. It is a permanent and necessary part of the reconstructive process. It has its distinctive value and is liable to certain characteristic fallacies. Both the good and the evil live and flourish in modern as in ancient thinking, though, as I shall argue later, it has been the function of modern thought to elaborate methods by which we can enjoy the benefit without paying the price.

2. To appreciate its exact function we must analyse with some care the general nature of the process, the conditions. of its validity and the limitations of its value.

The method, which for the sake of brevity may be called the Abstract, and in its more critical aspects the Dialectical, consists essentially in the endeavour to attain truth by the systematic interrelation of concepts subject to the test of self-consistency. It is the method of the bulk of mathematical and legal reasoning, while in metaphysics the 'Socratic dialectic' is its most brilliant example. Its value, its limitations and its dangers depend mainly on the subject matter to which it is applied, though partly of course on the hands into which it falls. In a general way it may be understood by considering its origin. In the formation of the empirical order connective concepts are formed by the precipitation of various elements of experience. The child's conception of a cat is formed and re-formed by many perceptions of soft strokings and perhaps of sharp scratchings, of purrings and mewings, by sensori-motor experiences of cuddlings and perhaps of chasings, by feelings of delight and perhaps of disappointment. The concept is not to be regarded as the actual endurance in the mind of faint remnants of these perceptions, nor does it consist essentially in their 'revival' in the form of images. We can hardly say even that it is a rallying-point of unity among many perceptions, for in a literal sense the perceptions change and pass, and the only strict unity among them is that of the mind through which they pass. Nevertheless, the concept is formed of elements 
given originally in experience. These elements are subjected to processes of analysis and synthesis whereby contents are formed, which as wholes do not necessarily resemble anything in experience. Thought does not copy. It builds. But it builds only with materials taken from the experienced order, and any apparent concept not formed from such materials is meaningless, and turns out upon examination to be no concept, but either a form of words or a mistranslation of something else. To this extent the content of the wildest imagination is related to and may be compared with experience.

But the concept of pure imagination and the concept of logic differ vitally in one respect. Both exist as contents of that mental state which we may call conception. But the imaginary concept, at least so far as it is recognised as imaginary, is satisfied, if I may so express it, with its own existence. The logical concept, on the other hand, involves a reference to some further reality. Now this further reality might be only another concept or system of concepts existing in my own mind or in that of other people. But it may also be Reality in the sense of that which is continuous and of one texture with experience-the Whole which is given fractionally in present experience. Now concepts in general are formed, as has been said, from this Reality, and the function of the concept in knowledgeand this function is what distinguishes the logical concept - is to gather together related elements of Reality. The logical concept then has an implied reference to Reality, ${ }^{1}$ and when this reference is justified by the existence of something Real that corresponds to it, the concept may be said to have Validity. ${ }^{2}$

1 Thus the concept may be regarded as embodying the material of an existential judgment, which only requires to be affirmed to be definitely asserted of reality.

${ }^{2}$ That is to say, a concept $A$ is valid if the judgment ' $A$ exists' is true. But may not a concept be valid on any other condition? The point raises the most difficult question of conceptual logic, the validity of ' imaginary expressions,' concepts to which nothing in experience conforms, but which are formed by logical processes and can be used without allacy in calculation. It seems impossible to deny all validity to such conceptions, but I think it may be said that their validity depends 
Now we may deal with our concepts, combining and re-combining them in numberless ways, without thought of any reference to reality or to anything whatever beyond themselves. This is the work of pure fancy. We may also deal with them with the purpose of gaining further knowledge of reality by means of our operations. This is conceptual thinking. But in so doing we may or may not act with clear consciousness of their ultimate reference. Without this consciousness we may go a long way in certain directions without error, while in other directions we are landed in serious fallacies.

For though, for the purposes of knowledge, the concept has value only through its reference to the real order, on the data and processes by which they are formed and the results to which they lead. If these conform to experience they are valid though the existential judgment founded on them is not true. Their validity is therefore dependent on other concepts to which they stand related, it is relative or extrinsic.

Validity is a wider conception than truth. It is the character of a datum or a process which yields truth, or, more generally, it is the cause or ground of truth. With false premisses a valid process yields false results, but they are results which would truly follow from those premisses. As applied to a perfected system of knowledge the two terms would coincide, as in such a system all the data and processes would be explicitly asserted in the form of propositions which would be true. In anything short of such 2 system valid processes yield truth only on the condition that they are free from dependence on any data or processes which are invalid. Conversely, true results may be derived from invalid processes.

The consideration of a perfect system of knowledge indicates that a valid process may be converted into a true proposition. Thus a concept which is intrinsically or unconditionally valid is one which may always be expressed as a true existential judgment. But an imaginary expression cannot as such be so converted. The process of calculation, however, in which it forms a part and by which we prove, say, that equation $\mathrm{A}$ involves equation $\mathrm{B}$, may be expressed in the true proposition 'where A is true B is true.' The imaginary expression then has validity in this and in similar combinations with other concepts, but not otherwise. That is, it has a dependent or conditional validity.

It may, of course, prove possible to find some 'real' meaning for all imaginary expressions, i.e. at bottom to find a sense in which they are applicable to the world of experience. If so, cadit quaestio, but in the meanwhile it is, I think, impossible (a) to deny all validity to such expressions, (b) to allow them any independent validity. They have validity only as links in a train or combination of thought. 
though it thus points beyond itself, and though its logical value therefore is wholly relative, yet, as we have seen, the concept is for our minds something definite on its own account. It is represented by a word. It forms the content of a distinct mental state. As such it can be dealt with, and under certain conditions legitimately dealt with, as though, so to say, it existed upon its own account, that is to say, without reference to the experience which gives it validity. It can be brought into relation with other concepts, and by this correlation a new synthesis may be formed, or as the result of a comparison, a new analysis may be achieved, the existence of distinguishable elements in the concept being for the first time discovered. By the interrelation of concept and concept, thought, in fact, moves in a world of its own and may even go on to create fresh worlds. Let us see under what conditions the process is legitimate and to what fallacies it is liable. For this purpose we must consider a little more closely how the mind operates with its concepts. We have spoken of synthesis and analysis, of combining and breaking up contents. But these phrases can only be applied to the work of thought in general on two understandings. First, we must understand that they express the logic rather than the psychology of the process. If a given concept A may be described as being formed of elements $B$ and $C$, it does not necessarily mean that $B$ and $C$ came before the mind one after another, that then they were somehow laid hold of together, pieced into one and so made into $C$. This would be at best a very rough and mechanical, and at worst a quite misleading description of the mental process. The phrase really means that the concept $A$ is a whole constituted by or resolvable into elements $B$ and $C$, that we started with these elements and that we have arrived, no matter by what precise mental process, at the whole. We have thus effected a synthesis. If, conversely, we had begun with the whole and arrived at certain elements, factors or relations involved in it, this would be an analysis. Secondly, we must understand that the term synthesis may be used to cover very different cases. In some elements may be combined without modifying one another. If I mentally 
add 4 and 3 , a new whole is formed, but 4 and 3 remain within it. In other cases the elements are modified by the union. The formation of the synthesis is in fact the modification of one by the other. So if I picture a colour as one that originally matched a given colour, but is faded, or think of a figure as similar to a given figure but on a larger scale, the resulting concept may be spoken of as a synthesis of elements, but only if we understand that here the process is not in the nature of a juxtaposition, but of a modification or transformation. With these understandings we may speak of the work of thought on its concept-materials as consisting in a synthesis and analysis, and may treat rules and principles of the transformation of concepts as themselves concepts applied to other concepts in the process and with the result of transforming them.

Now what are the conditions under which the transformations effected by synthesis and analysis are valid? Let us first be clear as to the meaning of the question. If I combine elements to form a whole, that whole exists for me, and any qualities or relations that I find in it exist for me. They are before my mind. They are as such parts of my present experience. But so considered they are taken apart from the reference which is a part of the concept in its full significance. The question whether the new whole has validity is the question whether the reference to reality contained in the concept is justified, whether if we make the reference explicit in a judgment affirming. the whole to be real that judgment is true. Now the judgment might be false because the original premisses were false. But this case is not for the moment in question. We are enquiring only into the validity of a certain process and we may put the question in this way. Supposing realities corresponding to two or more concepts, would they together form a whole corresponding accurately to the whole which my thought forms when it puts the two concepts together? The affirmative answer depends on two conditions. (I) The character of the whole must depend on the elements that form it and on nothing else. But this dependence is by no means simple and unambiguous. A triangle is a synthesis of three 
straight lines, but three straight lines do not necessarily form a triangle. They form a triangle only when brought into certain mutual relations, and these relations as well as the straight lines themselves are necessary elements in the combination that yields the triangle. Now on the one hand, it is very easy to overlook a condition of this sort, that is to say, to omit some of the conditions required to give to a combination a particular character, and this is one great root of fallacy in deductive argument. On the other hand, if the only synthesis that we can legitimately form is one in which all the elements are given, and if the 'elements' are to include all the parts and the relations in which they are combined, it is not easy to see how we make any advance at all. Do not the parts in their relation constitute the whole, and if so is not the whole already given when the 'elements' (which include that relation) are fully enumerated? How then do we make any advance in thinking? The reply is that the whole is not given when the parts are enumerated and the relation between them stated, but when they are actually combined. It is then recognised as something with a character of its own constituted by distinguishable and assignable elements. I may think of two straight lines diverging from a point and then of a third cutting them both at some distance from the point. When the process is complete I have my triangle, a figure with a recognisable character of its own constituted by the elements specified, which remain recognisably distinct within it. This figure moreover may possess some distinct qualities and present some distinct relation of its parts which belongs to it only as a whole$e . g$. the three angles of the triangle. But if this characteristic is a true character of the whole itself, and does not contain any element that is not part of the whole, it will be found in any other whole constituted in a precisely similar way, that is to say, it will follow from the synthesis of the elements as such.

We have now, accordingly, reached one of the general principles governing the valid synthesis and analysis of concepts. If two or more elements constitute a whole of a certain character precisely similar elements will be found 
to constitute a precisely similar whole, and conversely, if a whole can be resolved into elements of a certain character a precisely similar whole can be resolved into precisely similar elements. If this is true it will follow that if there is any real combination of elements corresponding to a synthesis which we effect in our minds, the whole which those elements form will correspond, and its characters will correspond, ${ }^{1}$ to those of our resulting concept. This will hold good subject to a condition to be noted lower down.

But this correspondence depends entirely on the assumption that the mode of combination is the same in both cases. If there is any possible ambiguity on this point there is opening for error. Accordingly sound and unambiguous mental construction is dependent on one or other of two conditions. (a) The combination may be so simple as to admit of no ambiguity. The clearest case is that in which the combination involves no specific relation of the parts, the bare fact of combination irrespective of order or mutual position being the only presupposition. Such a combination in the case of two quantities is sufficient for the purpose of addition, and addition like more complicated syntheses yields a whole with a character of its own, of which further things are found to be true, and which is not arrived at till the components are added. Subtraction is at bottom the converse operation by which a whole is divided into parts standing side by side. But furthermore, $(b)$ certain rules of synthesis with their results may be verified once for all and applied in any number of cases. Thus higher rules of computation can be educed from simple addition and subtraction. Further, certain axioms result from the general principles already laid down, and serve as principles in computation. We have assumed that when a certain result is arrived at by a process combining given elements in a given way, that result can be generalised. It follows, e.g. that if we start from similar concept-elements and con-

${ }^{1}$ In a more ultimate sense the principle depends on the axiom that relations which hold between certain terms as such hold between them universally. This, it will be argued later (Part II. Ch. II.), is one form of the final principle involved in the reasoning process. 
ceive them modified in similar degree, similar results must be arrived at - so that (in terms of magnitude) if they are increased equally or decreased equally or left unchanged, the resulting terms will be equal. Different conditions, conversely, must have different results. Thus the principal axioms involved in calculation are applications of the general principle that precisely similar syntheses of similar elements yield similar results, while the specific geometrical axioms appear to be generalised forms of certain simple combinations.

There remains a second condition of valid construction. The elements that enter into combination must have no effect on one another other than that contemplated in the rule of combination itself. This condition can never be affirmed a priori of physical things. Suppose that a body $A$ acted on by a force $P$ undergoes a certain acceleration in a certain direction. Suppose that when acted upon by $\mathbf{Q}$ it undergoes a certain acceleration in a certain direction. What will happen when it is acted upon by $P$ and $Q$ together? It may be that when we compare the resultant movement with that which would arise from $\mathrm{P}$ (say) acting alone the difference corresponds accurately in direction and magnitude with the movement which would result from $Q$ acting alone. In that case it may be said that the action of each force in the combination corresponds precisely to its action apart from the combination. Conversely, if this correspondence can be assumed it is possible by a geometrical construction to determine the joint effect of $\mathrm{P}$ and $\mathrm{Q}$ from a knowledge of their separate effects. Accordingly, wherever this assumption can be experientially justified we can apply such a construction in the prediction or explanation of phenomena, and this is what is effected with success in the whole realm of mechanics. But it may be also that $P$ and $Q$ are so modified by the very fact of their co-presence that there is no precise correspondence or no correspondence at all between their action in combination and their action apart from combination. In that case we cannot apply our geometrical construction to infer from the separate to the joint effect. We can do nothing without specific experience of the effect of the combination itself. More generally, 
deductive reasoning is often illusory because the generalisations on which it rests are not strictly proven, and the new combination may be precisely the modification in which they will not hold good. All intellectual construction is therefore at bottom of hypothetical character, and requires reference to experience to establish its premisses and test its conclusions.

3. Thus we can deal fruitfully with concepts, combining and analysing them without reference back to experience, provided always that the result of each operation depends on the elements entering into it and on nothing else, and provided further that the elements entering into combination have no effect on each other apart from that contemplated in the combination. Fallacies arise when ignoring this condition we attribute to physical things combined effects corresponding to our constructions without specific evidence of this correspondence. Fallacies arise also when the concept is modified unawares as it passes from one usage or combination to another. This happens the more easily because in most reasoning the concept is represented by a word which always will have many associations in virtue of which its meaning is elastic and tends to bulge on this side or that in accordance with the pressure or tension of the context. The concept may thus come to diverge materially from the experience out of which it was educed, and so yield false combinations or engender unreal difficulties. In particular, in the mere fact that it separates from experience and sets up as an object of contemplation on its own account, the concept undergoes changes of character which are a fruitful source of fallacy.

Fallacies of this order, arising ultimately from a misinterpretation of the relation of the concept to experience, underlie much metaphysical speculation ancient and modern. They are the source on the one side of Mysticism and on the other of Materialism. They form the make up of the legalistic and formalistic type of mind. It is therefore well to examine them with some care and to illustrate them with some detail, and in doing so we can draw all that we need from contemporary thought. For though-as I shall 
try to show here, and more fully in the next chapter-the modern movement is directed to a truer synthesis of thought and experience, it is far as yet from attaining its goal. It still works under constraint, dominated largely by the instruments which it has itself created, because it is not fully conscious of the how and the why of its own action.

The concept arises, as we have said, as a precipitate of experience. Experience comes to us as a continuous and concrete whole. If we are to do anything with it beyond passively receiving it, we must in our thought break up the perceptual order. In so doing we are guided partly by the physical continuity and discontinuity of things which divide the world of perception into distinguishable objects, partly by likeness and unlikeness of character which enable us to name the objects, their attributes, relations and modes of behaviour. Both these functions involve a certain grouping and re-grouping of the elements yielded by the perceptual order, and the basis of this re-grouping is an analysis which distinguishes elements and enables us to deal with them apart from the whole in which they appear. Now as long as the concept retains its living function of co-ordinating experience the separation is not misunderstood. But in proportion as it becomes an object of interest on its own account, and is separated from the experience out of which it arises, and from the world continuous with experience to which it refers, two tendencies are set up. In the first place, the concept is taken as real, or at lowest as the criterion of reality. However uncritically formed it becomes a mould into which our thought runs and outside of which we fail to think clearly at all. Hence all experience that will not fall within its four corners is dismissed as illusory, and as the concept has been uncritically formed, and is further liable to distortion in the very process of becoming independent, such discrepancy goes far and deep. The result is that experience is treated as a mere world of appearance while reality is identified with the world of ideas.

Thus the relation of thought and experience is inverted. But this is not all. The concept itself undergoes a devi- 
talising process. Separated from the living function of co-ordinating experience it hardens into a shell which is the more empty in proportion as its outlines are more rigid. It crystallises its contents, and indeed any distinguishable element of its content, into an independent object, and takes that object as it stands for something real. Hence it endeavours to separate what are really nothing but distinguishable aspects of one whole. Conversely, it merges into one concepts which though essentially diverse resemble one another under one aspect. It confronts the world of experience with dilemmas demanding that it should conform absolutely or not conform at all to concepts which are in fact derived only from partial characters of experience, and are never given except as qualifying or intertwined with others. Lastly, it crystallises fluidity and movement into separate elements with gulfs between them, wherein true movement is lost.

The first pair of these tendencies may be illustrated from the history of the concept of Identity. As a point of view from which to correlate experience this term has two distinct roots. It serves to hold together the object that has many attributes, that appears in different times and places, that undergoes certain changes and exhibits various forms of behaviour. As such it may be more definitely expressed by the term numerical identity or Unity. But the concept of Identity also applies to the several manifestations of an unchanging character, that is to say, to all the elements of experience which present an exact resemblance to one another. As soon as the concept is cut off from the experience to which it refers a blending of these meanings occurs. The two concepts collapse into the element which they have in common-the notion of a unitary centre of different contexts - and the character is thought of as an individual entity which persists and is numerically one through all its manifestations. This confusion is made into the logical basis of generalisation. The difficulty of arguing from case to case disappears, it is thought, for what truly belongs to the concept in any one instance belongs to it as a unity once and for all, and to deny it in any other case would be mere contradiction. 
Hence generalisation becomes a question of insight and even of intuition. It is a question of knowing what elements form part of a concept and what do not, and the attempt to form sound inductive canons is rendered nugatory. At the same time the permanence and substantiality of the conceptual world is vindicated against the world of sense, since the concept acquires the unchanging character which the empirical world has lost. On the other hand, the abstract conception of identity gives rise to difficulties. For $(a)$ the 'manifestations' of the concept differ even in characteristic quality. The redness of the rose is not the redness of the geranium. (b) The manifestation suffers change, the red of the rose deepens and fades and $(c)$ in the strictly conceptual order it is not the rose that is red. The quality or characteristic identity of the rose species is not the same character as redness, but both more and less. With this puzzle predication itself becomes impossible, and our ordinary ways of thought are triumphantly dismissed as pertaining to the world of illusion by some metaphysicians. Others with more insight perceive without perhaps deserting the conceptual method that it is our way of taking the concept that is at fault. Identity is in fact a concept formed from and applicable to objects that are in one way or another different. It implies some difference, and is compatible alike with change, with variety of aspect and specific differences of character. Bare identity, identity exclusive of any difference, is an abstraction within an abstraction. It is in fact a false abstraction, to which nothing corresponds, and to endeavour to fit an experience or a thought into it is precisely like trying to construct a curve which shall be convex without being concave. Thus by the separation of the concept of identity from the experience in which it arises two distinct fallacies arise. One is the confusion of two meanings of the term by concentrating on the point which they have in common to the neglect of their essential differences. The other is the formation of a wholly unreal and impossible category to which thought and experience are to be subjected, with the result that they are condemned as illusory and full of contradictions. 
So far the first two forms of fallacy. Let us consider next the tendency to 'harden' aspects or processes which in experience are interwoven into things which are mutually exclusive. Under the hardening treatment the common categories can be pitted against one another and shown to be mutually irreconcilable. Thus as long as the concepts of substance and cause are taken as self-sufficient entities, or as exhaustively characterising the real nature of certain entities, it is impossible to reconcile them. Substance is the abstraction of self-supporting existence. What is substantial as such is therefore unchanging, and if per impossibile there are changes within it they must be selfdetermined changes. What then is a cause? The concept of causality is that of necessity in interaction, and when the two concepts are put together we arrive at the idea of interacting substances, that is of self-determining things which are determined by one another-a stark contradiction. The possibility of a solution in which neither concept loses its value appears when we consider each of them as arising, uncritically in the first instance, as a rendering of certain elements of experience. It then becomes clear that to render reality as a whole intelligibly we must give due place to these elements, but must also recognise that each is only an element and not the whole of the truth. What is real is self-maintaining, but it is also a system of interrelated changes. The element of permanence in that system is its substantiality, the orderly continuity of its changing phases, its causality. The notion that a given object must either be 'a substance,' as we at first conceive the meaning of that term, or must be wholly insubstantial, is seen to be a false dilemma, and what is self-determining - whether, indeed, anything short of reality as a whole is self-determining - and what contingent on surrounding conditions becomes a purely empirical question. The concepts of substance and cause are resolved into the abstractions of continuity of real existence, admitting of correlated and consecutive changes of character.

4. We have seen how fatal the 'hardening' of the categories may be to the concepts themselves. We may 
consider, lastly, how it distorts our rendering of experience itself by transforming the fluid and continuous into a series of crystallised terms divided by the void. This particular trouble connects itself especially with the function of analysis. To understand the given we analyse, and the more we can analyse it down to distinct elements, and in particular, the more we can get hold of elements that are qualitatively alike, or are at least comparable, the more we can render the world in conceptual terms. We can bring in mathematics and substitute calculation for verification. In all this we are apt to forget that all analysis lays us open to the fallacies of partiality and incompleteness. We can truly say of a concrete whole that it has a certain character, although that character by no means exhausts its nature. The predication becomes untrue only when we overlook its partial character. The summer sky is blue. It is absurd to criticise the predicate as false because the blue is of a particular shade and of a certain degree of luminosity. But the judgment does in practice become false, and is, in fact, a fertile source of fallacy as soon as these qualifying circumstances are forgotten. Thus the artist who literally renders the colour that he sees, and in whose work it is perhaps impossible to detect a flaw to which a name can be given, may, nevertheless, produce a spiritless copy out of which all the beauty has departed. What is this spirit? Often-in the world of beauty, perhaps always in the present state of our criticism -it is something which defies, or has hitherto defied analysis. It is that which is left over when all the characters that we have been able to distinguish and to name are accounted for. Now there are some who hold that this incompleteness of analysis is inherent in our thought. So far as analysis can go, they say there may be science and calculation. But reality, or at any rate most important parts of reality, such as Life and Consciousness and Beauty, are in their inmost essence unanalysable, and therefore there will never be a science of them. I do not think that we should be hasty either in limiting the powers of analysis or in restricting science to its sphere. But let us agree so far that the fallacy of incomplete analysis is a fruitful source of disorder. Let 
us observe further, that this fallacy has two main forms. The crudest is that of simply taking the ascertainable part for the whole, e.g. the measurable desire for gain as the only motive that counts in the conduct of business. A more subtle and pervasive fallacy is that of the complementary partial analysis. Here the given concrete is resolved into elements which are in reality mere abstractions. They harden into independent entities, and, when the first that are so separated are seen to be inadequate, the missing elements are similarly precipitated and transformed to the requisite degree of self-sufficiency. The result is a reconstruction which is related to reality much as an exceedingly ingenious automaton to the living being which it simulates.

One great family of fallacies of this order arises in the effort to render continuous reality in discrete thought. The fixity which the concept needs in order to be easily handled as a unity in inference, contrasts with the actual continuity which experience yields. Hence, abstract thought will resolve a continuum like space into an assemblage of points, or time into a succession of instants, or motion into a successive occupation of positions. The point is the boundary of a line (or, what comes to the same thing, of a segment of a line), just as the line is a boundary of a figure. It has, as Euclid justly remarks, no parts and no magnitude, because it is not a division of the line, but an abstraction within it-the abstraction of its end or beginning, which can neither be perceived, nor strictly speaking conceived apart from that which begins or ends. But the point also figures as the goal of an analysis which would treat space as a whole consisting of separate elements. For this purpose, either it must receive magnitude which contradicts its essential purpose, or the spatial perception must be declared to contain a contradiction, and we get the Zenonian dialectics by which extension, motion, and, indeed, duration as well, are shown to be impossible. Modern mathematics yields a reconstruction on its own lines through its conception of infinity. No finite number of points arranged in order, each next to its fellow, builds up the continuous line. Only an infinite number can do this, and the infinity is of such a character that it breaks out between 
any two points, however close we may endeavour to take them. No point is next to any other, because; between every two points there is always another, and that is to say, there is an infinity. Now this account draws a just conclusion from its hypothesis, but the hypothesis itself is open to more than one interpretation. If we keep resolutely to the conception of the point as devoid of magnitude, no finite number of these zeros will lead us anywhere. But this result seems to be falsely interpreted if it is taken to mean that space is an assemblage of point-elements of which there is actually, in the shortest possible line, an infinitude. This conception would balance one fiction with another. The true interpretation appears to be rather that the point is the abstraction of position within a continuum, and that no summation of such abstractions will yield the continuum itself, but rather that in the smallest possible quantum of the continuum the abstraction could be repeated in an infinitude of different relations. With this conception I think we approach a genuine intellectual reconstruction of the sense-percept of continuity.

To reduce continuous magnitude to a form in which it can be subject to calculation, ordinary thought breaks it up into units, and the units readily become fictitious parts. As continuous space is dissolved into points, so time is conceived as a succession of instants, though there are no instants and no breaks between the end of one time element and the beginning of another. In the same way, motion is regarded as the successive occupation of positions, though the moving body, strictly speaking, occupies no position. However short the time taken, it is moving through space, not occupying a single position in space. Now for the purpose of calculation, the error involved in treating the moving body as occupying a position may be made as small as we please. In the same way, motion in a curve may be resolved into a series of motions in very short straight lines enclosed at very wide angles, and the error may be reduced below any assignable point, and generally the rate of a continuous change may be treated as the limit which we should arrive at by taking the differ- 
ence between two values, each being regarded as a value momentarily possessed, and by reducing the difference indefinitely near to vanishing point. This approximative method was, until recently, taken to be the logical basis of the calculus, which was therefore conceived as resting upon a fictitious resolution of the continuous into the discrete. In this resolution there was an unavoidable element of error which was harmless, because it could always be reduced below any finite magnitude, but served to show the ultimate incapacity of the human mind to grapple with the real by rational methods. Theoretically it could only be justified by the assumption of infinitesimal magnitudes, an assumption which could be shown to involve contradictions. More modern analysis shows that the calculus gives an exact reconstruction of the continuous depending on the distinction between the limit of a series of values and any actual value within such a series. The theory of the calculus defines the limit with precision and without assuming infinitesimal magnitudes, and proves that it is not the approximate but the exact and unambiguous measure of continuous variation. Thus, in this instance again it would appear that while the first movement of thought breaks up the continuous into the discrete, its final aim is to surmount this point of view with the fictions involved, and to equate its concepts with the continua which actual experience yields.

What is said of the physical continuum applies without essential modification to the continuous, or at any rate, exceedingly subtle and gradual variations of character which the real world presents. Here, again, common sense, guided by practical interests of correlation, gathers together a certain section of experience under concepts, which thus possess, not so much an exact fixity as a certain range of meaning. This laxity is intolerable to abstract thought, which, accordingly, selects some particular case and hardens it into a type, to which any new case must either conform or not conform. As an alternative, using experience but using it badly, it takes an instance falling under the concept, and (since the concept is assumed to be one and indivisible) asserts anything that it finds in this 
instance of the concept as such. Thus with the aid of dialectic on the one side and false analogy on the other, abstract thought confronts experience, as it were, with a number of alternatives, whereas the reality presents itself rather as something that moves continuously from one alternative to another. In this relation the legalistic type of mind commits its worst errors, and again the remedy is the closer correlation of the concept with experience. For the bare alternative, $A$ is $B$, or $A$ is not $B$, is substituted such a concept as is symbolised by a curve in which every variation of B to the limit of zero is contemplated, and advanced thought in most departments may be rendered by systems of such curves. The economy of thought begins with the discrete, but the back stroke of experience drives it to make its account with the continuous.

Another family of fallacies derives from the relation of whole and parts in the organic order. In this order a whole is never a mere sum of parts, but involves such mutual actions and modifications among them as will upset our calculations if we seek to reason from the parts as selfsubsistent entities. The crudest form of fallacy here is to take the sum of parts for the whole. A slightly more refined error is to take the organic character as an extra part added to the others, possessed of mysterious efficacy and acting in an ill-defined manner among the rest. Thus the behaviour of living beings has been partially resolved into a complex interaction of mechanical forces. One school in consequence assumes that it has only to pursue the same methods further in order to make the analysis exhaustive. Others crystallise the differences between mechanical and vital processes into a separate substance which interacts with body and perhaps has its seat in some problematical region of the brain. Others again infer somewhat prematurely, that the characteristic phenomena of life are hidden from our intelligence and can only be felt and perhaps made a subject for poetry or rhetoric, but never for systematic study. If we let ourselves be guided by experience, what we find is that the behaviour of living beings diverges from the mechanical model in that it is constantly adapted to the requirements of the whole. To 
ascertain the precise nature and conditions of this divergence then becomes a purely empirical problem, but to state it squarely is to recognise that the character of each and every part is modified by the whole to which it belongs. The analytic view which resolves behaviour into its ultimate elements has then to be corrected by the synthetic view which accounts for each element by its place in the whole. The peculiarity of the organic character lies not in one specific part but just in its wholeness.

5. At the present time there is no danger that the errors incident to abstract thinking will be overlooked. On the contrary, all the tendency is in the opposite direction, and insistence on the rights of instinct, feeling, emotion, and the concrete practical interest is pushed to the point of considerable scepticism as to the scope of articulate thought. The tendency in the hands of thinkers must be suicidal, for thought is nothing if it abandons the attempt to be distinct, connected and articulate. It may, indeed, be questioned whether all modes of reality can be articulately rendered. "Those who maintain the negative, whether on the ground of some inherent irrationality of things or of the limitations of thought, prepare for themselves serious metaphysical difficulties. But it is probable that the current tendency is based on the failure of analysis in certain specific instances, for example, in the analysis of beauty, in the reduction of life to mechanical elements, in the explanation of the religious sentiment in terms of experience. In face of any such failure, two opposed fallacies regularly find adherence. One party maintains the sufficiency of the existing analysis. Another, convinced of its inadequacy, insists on the discrepancy between the living reality and the deadness of abstract thought, and exaggerates it into a chasm that never will and never can be passed. The element of mystery, the dim halo of the uncertain and inarticulate, the obscure and the primal, is for this way of thinking just the one thing that matters. The attempt to explain, nay, even the attempt to state a meaning in frank and unambiguous terms is resented as a violation of the sanctuary. Thus popular thought wavers 
between mechanical abstraction on the one side and mysticism on the other, the one, to adapt a famous antithesis, relatively void, and the other blind.

In point of fact there always is in experience more than thought can render in articulate terms. This holds of a very simple experience. Even one of the colour sensations to which we referred above has a quality which it is difficult, if not impossible, to render quite perfectly in abstract terms. The green of the oak leaf is a green of a particular shade and quality. We express this quality as far as we can by calling it a darkish green, shading to a slight suggestion of blue when the leaf is fully out. But it is difficult to give it its precise quality without calling it the green of the oak leaf, which is after all a definition in a circle. 'Green,' 'dark green,' 'bluish green,' are, in fact, general terms which, with a varying measure of accuracy express the character of the colours that we see. By attention and comparison, by trained perception and analysis, we can keep on increasing this accuracy so that it approximates to the limit of the concrete sense datum. Hence the painter's colour vocabulary is richer, and more diversified with shades of perception, than that of ordinary language. As the process of analysis advances so the rendering of experience becomes more perfect, and the element of error inherent in the translation of experience into thought becomes less and less material. Naturally, the more complex and subtle the object which we are approaching, the more backward we are in this process. When we are dealing with something like the sense of duty in which a thousand subtle threads of feeling are involved, but which is always pre-eminently a unity and destroyed by any breaking up of its elements, the task of analysis is of far greater difficulty. When, again, we are dealing with the nature of life, we are attacking that which for the most part is only known to us directly by certain superficial effects. Direct observation of the inner processes fails, and any conception that we form can only be the result of a prolonged effort of synthesis applied to very diverse and always insufficient data. Lastly, when we consider religious conceptions, we are dealing with the 
entire attitude of men to life and the world, an attitude which is, in fact, the expression of their total heredity and their total experience-likely therefore, one may say, to be of all things the last to receive satisfactory shape in explicit thought, and yet incapable of taking distinct shape and performing its functions effectively except through the medium of explicit thought. In such a case as this, we might, indeed, seem in sorry plight, compelled to choose between inadequate formulae or an ineffectual vagueness, were it not that thought is not fixed but plastic, that it corrects its own errors, and if allowed freedom of movement, shapes itself stage by stage to the requirements of the reality which it seeks to interpret. Throughout the process of growth, both the parties to whom we have referred will have a measure of truth on their side. On the one side, articulate statement is necessary. if thought is to advance at all, and it is only when certain elements of experience are made explicit that we can begin to see how much remains. On the other side, the adequacy of any given analysis is justly subject to searching criticism, and the 'mother-sense' has a right to express and to maintain any dissatisfaction which it feels. But both sides have also certain natural tendencies to fallacy. Analysis takes the part for the whole, or forces complex and subtle experiences into the harder and simpler categories with which it is more familiar. Feeling, on the other hand; sometimes opposes analysis altogether, and at others solidifies itself into some explicit dogma or doctrine, the proof of which would really lie and could lie only in the province of thought. 'This is the most fruitful of all sources of confusion. The real force behind a dogma is a mass of feeling that has never been analysed, never left its home in the mother-sense. But this feeling is not so strong as to be happy without the appearance of evidence and reasoning. It spins such evidence and reasoning, accordingly, out of the first materials that come to hand, and invests the flimsy web with its own intensity of emotion. The only element of assured truth in the whole matter, as analysis disentangles it, is the feeling in the background. This feeling is so far entitled to respect that it belongs to 
the mother-sense, that is to say, it has grown up in response to requirements of the environment, but how it is to be interpreted in detail, is unfortunately not to be judged by the simple deliverances of consciousness in which it issues. That experience is narrow and incomplete is not a reason for ignoring it altogether, but rather for seeking means of extending it. That analysis is imperfect and may be fallaciously used are not reasons for reverting to uncritical dogmatism, but for supplying that which existing methods lack and correcting what in them is amiss. We shall come in the next chapter to the methods in course of elaboration in modern thought with these objects in view. We may here briefly sum up the results of our examination of the stage of conceptual reconstruction.

The first stage of philosophic and scientific thought may be considered as the internal development of the conceptual order. In some directions, notably in mathematics, and wherever the combination or resolution of concepts gives rise to new concepts without surreptitious modification of data, a considerable advance can be made on this method with very slender reference to experience. But the process as a whole is liable to fallacies arising from the conditions under which concepts are formed. They are derived from a limited experience, by an analysis of very variable adequacy, and unless critically used they tend to a misleading rigidity which distorts the truth. They are falsely used if treated as tests of reality, or as self-existent, or as containing their evidence in themselves, and this usage is the basis of the separation between the world of thought and that of sense, or between Reality and Appearance. Their uncritical employment, again, engenders a certain materialism when use is made of the most clearly definable conceptions as the measure of things, and by reaction from the method, to mysticism when the unanalysable elements of experience are endowed with a special sanctity and divorced from the conceptual order; to Dogmatism when that which is at best but obscurely felt is treated as though it were explicitly known, and to a dogmatism of negation when the partial character of analysis and of experience itself is ignored. While the 
metaphysical attempts to construct Reality out of categories fails alike in its idealistic and materialistic forms, the reversion to mysticism or Dogmatism is not justified. The defects of the conceptual order are not due to eternal and immutable limitations of thought, but to faults in its operation which criticism is overcoming. Stated generally, the aim of criticism is to effect a reconstruction of the conceptual order by taking into account the conditions of its development. This will be found to involve a corresponding criticism of experience, and the methods of using experience, and to point to that more far-reaching reconstruction of our world which is being elaborated by philosophy and by science in the modern period. 


\section{CHAPTER IX}

\section{EXPERIENTIAL RECONSTRUCTION}

I. VIEWED as a phase in the development of Mind, the elaboration of the conceptual order appears not as an end in itself, but as preparatory to a higher effort. It yields an ideal of truth, an instrument of reasoning, a self-conscious awareness of the mind's own operations in cognition. Over against the flux, the tangled ends, the disjointed fragments of experience, it has set up the conception of a reasoned coherent order. The next step is to find this order in experience itself, to trace within the flowing, shifting mass the broad and permanent lines of movement which render it an intelligible whole. This synthesis of experience is the goal of the movement which we have traced from its beginnings. To effect it there are required, on the one hand, the systematic and critical examination of experience itself, which, though begun in Greek antiquity, is the peculiar work of modern science ; on the other hand-partly as a condition of success, partly as consequence -new methods of organising experience, and close criticism of the functions of the Mind itself-a work in which both science and philosophy have had their share in the modern period. We must endeavour to seize the leading points in the complex movement.

With the death of Aristotle, the great period of constructive philosophy in Greece came to an end, not, we may take it, for lack of fertile, original and constructive minds, but because the work of the conceptual reconstruction of reality had been carried as far as it could go with the materials of experience then available. But Science 
still flourished, and the advance of Mathematics in particular continued until the social decay of the fourth century arrested intellectual development in the West.

While Europe slumbered and slept, the Arabic schools, under an impulse derived partly from India, partly from the débris of the classical culture, carried on the development of Algebra into a distinct branch of mathematical discipline, and by their chemical investigations gave new scope to experimentation. The contact with Oriental culture brought about the tardy revival of learning in the West, which, after spending three centuries in the assimilation of Greek thought, set forth on lines of its own. Nothing is more difficult than to state in any general terms the distinguishing features of modern philosophy, and nothing more false than the denial that it possesses such features. All the problems of thought and being mentioned above are problems for the modern as for the ancient, but the modern has, after all, carried these problems further back. It is doubtless easy to recognise in many attempted explanations by modern thinkers, errors and distortions which are avoided by Plato or Aristotle. Such things always occur when an earlier thinker of the first quality is stating relations or describing processes which a later one is striving to resolve into something more elementary. Yet it is rarely, if ever, true that the solution of a modern problem is to be found in an ancient thinker, though it is not seldom the case that we may profitably revert from the partial explanation to which some moderns have been led, to the more balanced and rounded expression of the facts to be explained as we may find them in some Aristotelian definition. It is not to our purpose to deal with successive syntheses of philosophy or the value of the solutions which they have propounded of the problems of Reality. Our purpose is to note the emergence of certain conceptions essential to the work of Reconstruction, which we have seen to be the problem set to thought by the conditions of its evolution. Now, the leading conceptions required appear to be three. In the first place, the Mind must appreciate its own position. It must recognise and measure its own contribution to the 
world in which it lives and which it takes prima facie as plain reality. We may speak of this as the evaluation of the subjective factor in Thought. In the second place, we must find some elementary data, which at the end of criticism may be taken as a basis of reconstruction, and some method of building upon them. If these data and methods could be taken as final, that is to say, as independent of any of the limitations affecting Mind, they would be all that we should require. But as we cannot escape from our own minds, as criticism is still a mental process, and the most fully critical method a mental product, and the most objective 'datum' something that Mind can grasp, it follows that any reconstruction that we can make remains liable to the imputation of relativity. We shall need therefore in the third place a theory of rational proof which will decide on the claims of rival constructions, and furnish a grounded judgment of the final value of our thought-structure.

Of these three questions, the first two concern the nature and method of reconstruction, the third its final validity. To show how the first two arose in modern thought will therefore suffice for our present purpose, while we may reserve the third for later investigation from a somewhat different point of view, only touching on it incidentally here.

2. Now, the evaluation of the subjective factor in knowledge may be fairly regarded as the central contribution of the first movement in modern philosophy-from Des Cartes to Kant. Knowledge is the property of a conscious subject. It is conditioned by the character and limitations of its subject, and the problem of its relation to its object at once begins to press. It is here that the distinctive features of modern philosophy emerge. Ancient thought had been familiar with the conception of the mind as a structure interacting with objects, and Protagoras-if the Theaetetus reports him accurately-educed therefrom a sceptical form of relativism, making man the measure of things. Modern thought is an effort to work through relativism to objectivity, by accepting the 'moment' of 
relativity to reconstruct the reality which transcends it. It works down to an analysis of cognition in which the content of any cognitive act is regarded as involving a reference to something beyond the cognitive act itself, and having, therefore, prima facie, a separate existence. But as, under another aspect, the content appears to be identical with the total character of an act or state of consciousness, the question that pressed was how any reference beyond could ever be justified. At best the appeal could only be to some other content, and we might move in a world of such contents in the whole of which the apparent external reference might be quite illusory.

In fact, the result of the first stage in the development of the problem was the Humian scepticism, in which the fabric of knowledge was reduced to 'impressions' and 'ideas' devoid of valid reference beyond themselves. Reconstruction was attempted, in the first instance, by a criticism of Objectivity. It might be that all that we could know lay within the circle of our consciousness, but that within this sphere there was an immutable order which might be rationally apprehended and become the content of science, or irrationally and arbitrarily conceived and so form the content of mere opinion and error. This solution, already put forward in principle by Berkeley, is worked out by Kant on the basis of a theory of the contribution of the mind, not merely to our way of thinking about experience, but to experience itself. The underlying elements of the empirical order are now brought more fully into view. Complex elements are revealed in the apparently simple data of perception, and the structural categories are argued to be not merely results of experience but principles implied in the formation of that order which at first sight we take as simply 'given.'

But with this conception of objectivity Kant deliberately, if inconsistently, combined a different theory of reality. Things in themselves were unmodified by the work of mind, and though they could not come within the grasp of ordinary cognition, something of their fundamental character could be apprehended by analysing the postulates of the moral consciousness. This dualism broke up the 
Kantian system, but observe, meanwhile, that the Critical philosophy yielded a theory of knowledge as at once relative and objective. Knowledge not only was but was meant to be a mind-structure, and the mind-structure was resolved into a number of elementary functions working in systematic co-ordination, not merely to connect one empirical result with another, as in simple common-sense psychology, but in the very tissue of experience itself.

The natural outcome of this conception was to enlarge the borders of the mind-world and make it co-extensive with reality. The operation of 'things in themselves,' inconsistent as it was with the general postulates of knowledge, was eliminated, and the objectivity of critical thought was vindicated, because outside the world of mind nothing could exist. But the more the world was identified with the mind-structure the clearer it became that that structure passed through phases of development. Limitation, error and evil could not be denied, and on the general presuppositions involved they could only be identified with some partial phase of the mind-world. Accordingly, the thought-categories are now exhibited as a development ascending from what we may call an inorganic stage in which partial truths are so held as to conflict with and destroy one another, to a comprehensive synthesis devoid of internal contradiction and complete in itself. Objective truth is now in the whole seen as a whole, or, more accurately, in that final vision the antithesis of subject and object is overcome.

The idealistic solution was based on a one-sided interpretation of the problem to be solved, for, in fact, the reference of cognition to its object implies a duality which could only be destroyed by annihilating cognition itselfas many mystical modes of expression show clearly enough. Weighted with this one-sidedness, the monistic interpretation becomes involved in insuperable difficulties as to the nature of error, of evil, and even of individuality. But it forwarded the general movement of thought, especially in the form of the Hegelian dialectic, by conceiving the Kantian mind-structure as a development, by emphasising the relative and partial nature of the categories, and by 
tracing error to its root in the mistake of part for the whole, and indicating, accordingly, where truth lay. That notwithstanding all the errors of one-sided thought and partial experience, truth lies in the complete system of inter-connected experience,' and that no further test can have any meaning is thus made clear, and we get in the growth of the mind itself the double conception of a relative truth always in process and always involving error, and an absolute truth already present as the moving force in growth, but becoming known in its fulness only when the process is complete.

It is possible to retain this conception of truth without that identification whereby the subjective factor is really made to swallow the objective. If thought involves reference to an object, a system of thought is a system of such references. Where such references are mutually inconsistent there is demonstrated error. Where they are mutually compatible there is no reason to impute error. But they may be not only compatible but mutually necessary. Thought may in the end stand as a system of references of which all the parts imply one another and which excludes every basis of negation. It is in accordance with the idealist conception that in such a system there could be no rational ground of doubt attaching to the references which it contained, for any such ground must itself be a thought arising within the system of thought which has been held to exclude it. Admit, in fact, that belief or doubt alike arise within the circle of operations of thought in or with experience (phrase it as you will), admit that the appeal is always to further operations of thought, and the conclusion is that a completely articulated system, including all operations of thought with experience, can and need appeal only to itself. I shall dwell on this point further at a later stage and consider its bearing on the actual structure of our partial knowledge. For the present I have to note its value for the conception of our thoughtworld as a structure in process of growth. If we hold fast to the distinction between thought and the thing thought about, we are led to the conception of any thought as being necessarily a function of two independent things-a mind 
and a wider reality of which that mind is a part and with which it is in contact. We see next that any thought which we can justly call knowledge is supposed thereby to be an accurate assertion of such reality, and that if this supposition is ever justified, it implies that the mind operates somehow so as to correspond with reality. It is clear that such operation is only one of many possibilities, and among other things the questions, how it has come about and to what extent it has come about, are distinct but related questions affecting the whole interpretation of that which we take for knowledge. We have to recognise that the most complete and consistent thought attainable by any mind will contain only so much truth as the measure of that mind permits, which may be anything from zero upwards. Truth itself is not relative, but the truest judgment we are capable of making is related, in strict proportion, to the structure of our minds. Our attitude to our own apparent knowledge becomes not so much one of assured confidence as one of effort and conscious imperfection. Our most fundamental conceptions become ways of apprehending reality or of co-ordinating experiences that have lost all sacrosanct immutability and may require revision and supplementation like everything else that belongs to growth. The structural principles of thought are conceived not as rigid moulds into which all truth must fit, but rather as plastic elements of a growing structure which may be modified without loss of identity to take a wider and fuller experience within their grasp.

3. Thus, on the one hand, the realisation of the subjective factor in knowledge leads to the conception of a mindstructure with a life history of its own, a conception which gives shape to the modern investigation of personal and social psychology. On the other, it engenders the logic of experience. It demanded some form of mental operation in which an objective element could be securely predicated. Of such a form immediate experience seemed to be the clearest case, and experience has been the term round which the controversies of philosophy have raged. For 
at first sight we seem in sense-experience to be in direct contact with outer realities, and if it were so, we had here so firm a basis of knowledge that the only question to be discussed would be the method of building upon it. The Berkeleyan criticism soon showed that the matter was not so simple, but even so it left experience standing as so much fact, though fact of an 'internal' psychological kind, and the problem of knowledge was to understand how thought so organised experience as to discover general truth. The Kantian and post-Kantian analysis showed, however, that 'immediate' experience could not be taken as a simple datum. From the outset we assert, and though in sensation or in any form of immediate consciousness that which we assert may, in a certain sense, ${ }^{1}$ be taken as fact, this sense is only reached by an effort of abstraction. Analysis showed that the 'pure experience' which seemed a prime starting-point was an abstraction from which the elements of reference that piece its parts together are omitted. The true starting-point of knowledge is the assertion which assigns an object a place in a permanent order, whereby it enters into relation with other objects. Yet-and this is the paradox of knowledge-this order is itself built up by slow degrees and is certainly not an object of thought until experience is far advanced. The solution of the paradox is that the cognitive life of mind is from the first a correlating activity which connects the successive phases and weaves them into a plastic order to which every new experience is referred. It is true that the precise nature of the reference is determined by relations which are contained in the objects of experience when experience is taken as a whole, but $(a)$ since experience comes in fragments, spread over time, to take it as a whole is only possible for a mind which can correlate distinct data, and (b) certain methods of correlation, viz. those which involve generalisation are never 'given,' but involve assertions going beyond anything that can be given.

Thus the unit of knowledge is an assertion involving the object in relations, and the 'pure' experience which

$$
\text { I See below, and footnote on following page. }
$$


may be taken as so much 'fact,' is the asserted object denuded of these references. ${ }^{1}$

Objects, then, are not 'given' in experience from without in the simple manner at first supposed by common sense. Hence, even if we assume perception to be an accurate assertion of an outer object, we no longer conceive the one as a photograph or impression of the other. We conceive it as a construction or, if we prefer the term, a reconstruction out of materials of stimulus and psychophysical process in which there is no likeness to the object at all. This criticism of experience may be taken as the starting-point for two very different lines of investigation. On the one hand, it is the point of departure for the investigation of the psycho-physical processes underlying experience. For, if experience is still the basis of knowledge, it is, genetically considered, a mere effect of the specific reaction of certain complex structures under given conditions. Ultimate in the one sense, it is derivative and relative in the other. On the other hand, the recognition of the reference involved in the bare assertion of objects of experience opens a door of escape from subjective idealism. The problem of knowledge becomes that of verifying these references, and the mode of verifying them is by thorough-going interrelation with one another. This interrelation is the work of the correlating or, as Kant called it, synthetic activity of thought. This he showed to be an original function, with its own appropriate modes of operation, without which no organised body of experience could be formed. ${ }^{2}$ When these modes become con-

'I 'see' a figure over there. Investigation convinces me that it was an illusion. The so-called 'seeing' is a false judgment, what convinces me that it was false being at bottom inconsistency between it and other judgments, i.e. the impossibility of correlating it with other objects. Nevertheless, as a mere object of immediate consciousness, i.e. apart from its reference to a point of space outside my body, the figure was real. As such it was 'pure' experience or the object of simple apprehension.

2 To adopt this general result of the Kantian criticism is not of course to accept his description of the modes of operation in question or to distinguish between what is 'given' and what is not given on anything resembling Kantian lines. 
scious they are stated in abstract terms, and figure as axioms. In this analysis Kant gave the first critical account of the nature of axioms. For the axiom rests not on apparent self-evidence, the psychological feeling of certitude, but on the correlating function which it formulates in general terms. We shall return to this point later. Here we may be content to remark that in the modern philosophical movement from Kant onwards we have criticism attacking the systematisation effected by thought at both ends. We have it applied alike to the primary data and the supreme correlating principles. Kant himself was clear that these principles have no validity and no real meaning, except in relation to that which is given in experience, and his criticism-as distinguished from his quite inconsistent reconstruction-has so far the same tendency as that of the British empiricists. Thought is that which has the function of correlating experience. What is true is in the last resort judgment based on a duly correlated experience, and thought is the function of correlating experience. There was needed, accordingly, a logic of experience, or a scientific induction, and to elaborate such a logic is as much the problem of modern as the formation of a deductive logic was the task of ancient thought.

The evolution of the modern theory of method, however, has not been determined by philosophical impulses alone. The revival of experimental science preceded the modern movement in metaphysics, and the development of mathematics engendered new methods of handling experimental results. In particular the discovery by Newton and Leibniz of methods of calculating from rates of change to results, or vice versa, gave an enormous extension to applied mathematics. It became possible in physics to assume the action of very simple forces, to calculate the result of their operation in complex or remote cases and to compare the result with accurately measured observation. This is the method, not devoid of liabilities to error, which has on the whole determined the advance of physics. In fields where simple and measurable forces could not be so assumed and where observation at first 
floundered in a morass of contradictions and confusions, mathematics at a somewhat later stage came to the rescue with the theory of Probabilities, and modern science relies on statistical evidence, on the one hand, for the first introduction of order into newly reclaimed territory, on the other for the correction or verification of its calculations from hypothetical principles. Scientific induction, calculation from hypothesis, and statistical verification are thus the characteristic methods of modern science, and all are governed by the principle that truth is to be found where results coincide - the principle of Consilience, which underlies all modern methodology. To this it must be added that from the invention of the microscope and telescope onwards there has been a parallel extension of the world of observation itself. Outer experience no longer means the world of the unaided senses. It is a supersensible world, and in as far as chemical and other experiments enable us to see in laboratories that which common experience never reveals it is also a supernormal world. It is a world whose data ramify in all directions far beyond the experience of common sense.

4. The problem of modern science in its most general terms has been commonly stated as the ascertainment of the laws or general relations of coexistence and sequence among phenomena. The term phenomena suggests metaphysical implications which are open to criticism. But if we overlook these for the moment we may take the formula as a statement of the problem of knowledge in its simplest terms, viz. as a correlation of the elements of experience. Now many relations are given in experience, and the function of thought is to use these as data for the discovery of further relations which are not and perhaps cannot be given. On the basis of the given relations thought builds up the conception of a reality continuous with but extending indefinitely beyond experience, containing and explaining the order of experience as a part of itself. It is in this sense that the function of thought is the correlation of empirical data, and this function is primary, that is to say something that thought 
contributes. But this phrase is easily misunderstood. It does not properly mean that the work of thought is to construct relations which would not otherwise exist. For the function of thought as a correlating activity is to discover what is already real, and the only thing it constructs is its own system, which it means to correspond to the real order. The proper meaning of the phrase is that thought does not find all the relations that it needs given in experience ready to hand, but is an impulse to find relations which exist but are not given, and to discover a complete connectedness where only a partial order is observable. The Logic of Experience seeks to lay down the principles and conditions upon which this process of correlation is valid.

Of this logic we shall have something to say in the next part. Here we note the terms in which the problem is stated as a characteristic product of modern thought. We may usefully contrast the question, "What are the relations between this and that datum?' with the 'What is it?' which is the characteristic formula of antiquity for the scientific enquiry into a subject. The older form of enquiry tacitly assumes that there is some typical conception under which the subject can be brought, and which when fully set out will contain the explanation of any of its properties. The thought of antiquity, that is to say, is guided mainly by the impulse to reach certain central conceptions, capable of being stated as definitions from which a number of properties may be deduced. The order of nature, including man and society within it, is seen as an array of types to which actual things approximate. Science is the knowledge of the central essence of the type, and of the properties derived therefrom. So far as actual things diverge from the type it is because they contain elements of ambiguity and indefiniteness which remove them from the purview of science proper, for science deals only with the necessary and the universal. This is not necessarily a static view of nature, for, as in the system of Aristotle, the types might form an ascending series, and the world might be conceived as a process in which the higher types are realised in succession. But it is a view which places the typical, the complete, the definite as it were on an eminence, 
and is ill adapted for the systematic study of order in variation. It is, in fact, quite consonant with the conceptual logic, and formulates the range of truth that can be studied with a relatively slender experience and without constant back-reference to experience. The modern problem places the whole field of enquiry more on a level, its uniformities are sought through all the wilderness of variation and change, and its types are rather sign-posts or meetingpoints or critical turnings in a continuous area than solitary eminences parted by the void from one another. Its clue is the discovery of an order of which all the terms are comparable inter se, with which our experience, with its rich qualitative diversity, can be correlated. Thus our sensations of sound, light, colour, heat, our experiences of touch, resistance, pressure, our perceptions of motion, rest and bodily form can be correlated with the terms of a mechanical system which thus carries the notion of a single order right through the world of perception. Indeed, the success of the mechanical principle in its own sphere tempts to a hasty generalisation which would extend it to the whole of reality, but a very little philosophical criticism is needed to show the fallacy of baldly identifying the life of mind with a process to which it stands related. The further effort of modern thought then is to find a similar order for the world of mind and of life in general, and for this purposethough as yet the work is but beginning-it has elaborated the comparative method and the governing conception of development. If the mechanical order was the culminating conception of the first movement of modern thought, the evolutionary order holds the same place in the second period, and as the mechanical system provided the common terms by means of which all the variety and change and detail of physical experience could be brought into correlation, so the idea of development enables the facts of structure and function, of life, intelligence and purpose to be seen in their mutual relations. Experience falls into the two series, the mechanical and the developmental, or, as I will venture provisionally to call it, the Teleological. There will remain the final problem of interrelating the two orders, a problem which can never be wholly solved 
until the two terms of the relation are completely understood, but which it is constantly necessary to state and re-state in the light of the best available knowledge.

5. To many people the march of science seems to narrow the world. The truer view is that it has enormously expanded our conceptions of what is possible in Reality. Hence it is that partly as cause, partly as effect, but altogether in sympathy with the lines of movement already sketched, the idea of the Infinite plays a central part in modern philosophy. Modern thought may almost be said to have reversed the attitude of man to this idea. When the Pythagoreans ranged the One, the Finite and the Good on one side, and the Plural, the Unlimited and the Bad on the other, they expressed the characteristic feeling of the Greek thinker and of the Greek artist. Order, proportion and all that we now call organic unity were the essentials of the Greek ideal. They emerge out of the formless as Aristotle's specific forms arise out of shapeless Matter in its impulse towards the divine. Growth is necessary to them, but necessary as a means. It is valuable only on the way to perfection, which once reached, what need of further growth? Now this static perfection is almost intolerable to the modern. It bores him like the mediaeval heaven. Movement of itself has become part of the ideal. The fragmentary, with its suggestions of something vaster, the 'broken arc', the tattered banner of the forlorn hope have a greater charm than the rounded whole and the polish of perfection; as the gloom, the half lights, the long vistas of dim unending Gothic aisles appeal with a force which classical symmetry can no longer match. The contrast has been stated once for all by a master whose sympathies with both sides were keen and perfectly instructed.

To-day's brief passion limits their range

It seethes with the morrow for us, and more,

They are perfect. How else? They shall never change.

We are faulty. Why not? We have time in store.

What we know and do is a living fragment whose fibres and tendrils stretch out into an immensity beyond, and all 
that suggests this beyond, be it even failure, sin and suffering, is to us more than the lovely thing of which we see the end. ${ }^{1}$

None the less, there remains the demand of reason and knowledge for wholeness and completeness. Reality is infinite, yet we desire to understand it as a whole. But how can the infinite be a whole? How can it be completely understood without being summed up, and how can it be even potentially summed up unless it be finite? It is not the bare conception of the Infinite which gives rise to the Kantian antinomies but the endeavour to unite the two conceptions of the Infinite and the intelligible order in the idea of an. Infinite whole. I shall touch on the question again at a later stage. Here it is only necessary to remark that once again in the conception of all our experience as finite and yet as having roots in the Infinite, we have the distinctive modern view of the world of human thought as relative and yet capable through self-criticism of transcending its own relativity, and relating itself to the vaster whole of which it is only one facet.

This conception again has its justification in the idea of development. For as applied to knowledge the theory of development explains the actual limitations of the mind by the conditions of its genesis. It shows that adequate adjustment of response to environment being a sufficient condition of survival, a psycho-physical structure may be blind to everything but just that which is necessary for such adjustment. But it also reveals an indubitable growth of faculty, and, what is most important, the emergence of powers and interests unconnected with mere survival and concerned with the expansion and-improvement of life.

I "Euclid always contemplates a straight line as drawn between two definite points.... He never thinks of the line as an entity given once and for all as a whole. This careful definition and limitation, so as to exclude an infinity not immediately apparent to the senses, was very characteristic of the Greeks in all their many activities. It is enshrined in the difference between Greek architecture and Gothic architecture, and between the Greek religion and modern religion. The spire on a Gothic cathedral, and the importance of the unbounded straight line in modern geometry are both emblematic of the transformation of the modern world." Whitehead, Introduction to Mathematics, p. 119. 
It thus indicates that the limits of mind at any given moment are no adamantine barriers, but rather that the boundaries of its operation at any given moment are functions of its development at that moment, and are perfectly capable of extension. It prepares us for the view that by recognising our limits we transcend them and that by knowing a truth to be true only for us, we know it absolutely. The final secret of Reconstruction lies in the consciousness of development itself.

6. We see then that the world of advanced thoughtthe world of philosophy in the older and more legitimate sense in which that term included the sciences-is one in which common thought has undergone a fundamental reconstruction, both in its methods and its data. We have to picture common sense advancing on uncritical lines and building up an order of ideas which has its value but is by no means a perfect mirror of reality. We picture criticism beginning with a sense of this deficiency, with the notion of a real world set over against this mental construction. Such an opposition we saw is implicit in the higher religions and is posed as a definite problem for logical solution from the first hypotheses of the PreSocratics onwards. Ancient philosophy defined the problem in general terms, and modern thought, with its emphasis on the subjective factor, has traced the difficulty to its root, and with its new methods of reasoning and observation has made some notable advances in the work of reconstruction. The essence of this reconstruction is the entry into the sphere of consciousness, previously concerned only with results, of the data and the processes by which results are obtained. This critical movement begins in the ancient world in the demand for a logical treatment of the conceptual order, with the ideals of unity, system, accuracy and interrelation, with the exposition of the formal conditions of a perfected science. We have here the general conditions of metaphysics and of mathematics, at least in the form which they assumed in antiquity. Indeed, the Elements of Euclid remain the nearest approach to the realisation of this ideal of conceptual reconstruction. 
In modern thought the critical movement is carried a stage further by the resolution of the processes involved in knowledge into their elementary factors. If the concept is treated as a function, having its meaning and value in the order and correlation which it establishes among the data of experience, the deliverances of experience have in turn to come up for criticism and submit themselves to the criteria of consistency and coherence imposed by thought. Fixed starting points and absolute principles are replaced by partial views, experimental assumptions, working postulates, which are to be tested by being brought together, and are ultimately confirmed, modified or rejected according as they can or cannot conform to the requirements of a coherent whole. Thought thus becomes a plastic structure subject to constant modification, at any time conditioned by the existing stage in the development of method and by the acquired mass of experience, but constantly through growth overstepping its conditions and expanding as well as tightening its grip. In this conception, while results are resolved back into conditions, the data, the processes, the principles which underlie them, these conditions are also viewed in relation to the results on which their coherence or incoherence, their breadth or narrowness of scope becomes manifest. The foundation of the movement, then, may be described as a correlation of the conditions, the data, processes and principles of thought and experience with their results. This correlation is not altogether the cause nor merely the effect of modern science, but is involved in it through mutual interaction, expressing its tendency, and consciously, half-consciously, or unconsciously shaping its efforts.

We have then a double movement of Reconstruction or of the conscious and reasoned effort to obtain knowledge of Reality. The first is essentially a Conceptual Reconstruction, and though it involves criticism of mental process and of logical validity its principles are mainly those which arise from analysis of a conceptual order as such. The second is essentially an Experiential Reconstruction, and its criticism involves what we may call briefly a correlation of Mind products with their conditions. 
The survey of these conditions carries us right through the field of experience and includes therein the history and structure of mind itself. Its aim is to set the organised experience of the race in its right relation to the system of Reality, showing on the one hand how it has grown up, on the other, seeking to determine the extent to which it enables us to judge of Reality as such. While the task set in these terms is infinite as Reality itself we may consider the mind as fairly entering on this phase at the point at which, through the aid of the several movements that have been mentioned, we are able to take a view of the world of our thought as a growth resting on assignable conditions and capable of extension, through the intelligent appreciation of those conditions.

This is for thought a new kind of self-consciousness arising gradually in the course of history and realising itself rather through the collective operation of many minds than by change of any innate quality of individual minds. None the less, it involves a new orientation, a change of attitude and direction not less fundamental than that which is implied in the dawn of self-consciousness in the individual. The change is quite parallel to those which we have noted at earlier stages. As the massive experience of the past determined the reaction to present stimulus in such manner as to avoid a pain or procure a satisfaction before the pain or pleasure entered into consciousness, as the pain or pleasure entered consciousness and determined action in similar cases, though without consciousness of similarity or generalisation, so lastly, general relations operated as explicit grounds of inference without any consciousness of the principles of method logically involved. And just as the pain or pleasure rose into consciousness as an end of action, and as the general relation that connected different experiences became known for what it was, so finally do the principles underlying generalisation or any other inference come into the conscious area. The advance is always in the same direction, the underlying forces guiding effort are brought into relation with one another and with those that are already known. Every such movement involves a certain 'turning of the eye of the soul,' a new direction 
of the correlating activity which constitutes the function of consciousness and in that sense a change of quality. The turn by which the mind becomes aware of its life as a unity is what we call the dawn of self-consciousness, and distinguishes the human from the animal mind. The turn by which the mind of humanity reduces the structure of its thought to its elements to reconstruct its view of reality from the foundations is a quite comparable advance in selfknowledge. Finally, each 'turn' of consciousness reveals a deeper plane of reality. The world, which is for the lowest intelligence nothing but a disconnected series of sense-stimuli, becomes first a network of related objects, then an order of beings persisting through change, and like amid unlikeness, and lastly, a system of forces and principles, mechanical, spiritual or other, whose interplay determines the superficial changes of the shallower plane.

If we conceive the critical movement carried to its completion, we shall have reached a central point from which, in outline, the genesis, the development, the conditions of Mind in man lie open to view, and with them its potentialities and, we may say, its future. The entire history of Mind may be said to lead up to this point, at which it becomes, as we have put it, self-conscious. The question that now arises is how far this self-knowledge yields selfcontrol, how far, that is to say, having gained this point of view, the Mind could not only forecast but shape its future. To answer this question we must turn from the development of thought to that of action. 


\section{CHAPTER X}

\section{THE WILL IN DEVELOPMENT}

ONE source of confusion in Ethical theory has been the close relation of distinct aspects of ethical life. Happiness, self-realisation, personality, the common good, virtue, duty, conscience, moral sense are all distinct conceptions, but they are not so readily to be assumed as independent factors in the life of man in society. They are terms expressing certain distinguishable elements in an ethical experience which is, after all, at bottom a unity. And in this unity all the relevant elements are closely interconnected. It is possible, accordingly, to start from any one of these conceptions and make it the centre of ethical theory, but in its further development such a theory has before it one of two alternatives, either to fall into hopeless one-sidedness or to take up into itself in bulk the content of theories that start from the remaining elements. Hence, while different in form, ethical theories tend, as they fill out, to cover very nearly the same ground. On the theory of development this result is very readily intelligible. For, in the first place, the function of ethical theory is to harmonise a number of functions that have grown up in unconscious and incomplete, but nevertheless in real and fundamental relation to one another. In the second place, as ethical development consists in an evolving harmony of feeling and experience, the problem of theory is essentially to reconcile and not to exclude. It will, accordingly, now appear that each of the main types of ethical theory has its place in the evolutionary scheme. Happiness, for example, is the harmony of feeling with feeling and of feeling with 
experience, which is the general character of the good. Such a harmony, if attainable at all for the individual, is so only because the self is a potential system in which, by a duly proportioned development of each several element, a harmonious working of the whole is possible, and such a development is self-realisation in the strictest sense of that term. But, again, for the rational mind there can be no satisfaction in a harmony that anywhere involves fundamental discord. The rational impulse is an impulse to harmonise all that is susceptible of harmony, and that is the whole world of sentient mind. Hence, for the rational man there is no harmony within the self unless as a basis. of harmony with other centres of experience and feeling, and the realisation of any one self is regarded only as an item in the development of society, that is in a Common Good. This development implies an ideal of Personality in which the moral virtues as well as the intellectual and physical excellences are constituent conditions, and the promotion of which, when it conflicts with any warring impulse or interest, is felt by the individual as a duty. Finally, the instinctive or quasi-instinctive promptings that urge us without reflection to the action generally necessary to such a harmony, form the content of the moral sense, and the summed up judgment of present duty, in which elements of direct feeling and rational reflection blend in a final deliverance which in foro interno is felt to be supreme, is the reality to which the name of conscience has been given.

We have to follow briefly the development of this system of practical rationality in its point by point correspondence with the general evolution of mind.

\section{The Hereditary Factor.}

Of Ethical as of all conduct the primary psycho-physical basis is instinctive. Nor is there a whit more difficulty in understanding the origin of social instincts - that is, of instincts tending to foster a common life and to ensure the maintenance of the species - than of instincts directed only to the maintenance of the individual. Whatever the source of variation in the first place, it is evident that varia- 
tions of function tending to racial preservation would have an even better chance of survival than variations tending only to the preservation of the individual. Accordingly, from an early stage-indeed in a sense from the very lowest-heredity builds up structures, which in response, partly, perhaps, to internal changes, partly to definite outward stimuli, lead the individual to mate, to produce and perhaps make elementary provision for the young, and finally to consort in many cases with others of its kind. These instincts, on close examination, reveal the characteristic limitations, defects and individual variations of structures that have been roughly shaped to their work by the indirect action of heredity. Thus, the infant mammal has an instinctive impulse which is satisfied by the sucking of the breast, but does not unfailingly and unaided lead it to find the breast. It follows its mother, but its initial impulse is often to follow any large slowly moving object. A sparrow will feed the cuckoo that has expelled her own young, because she cannot resist the sight of a callow nestling and a bill gaping for worms. The fabric of instinct as a series of responses to stimulus is well seen in the cries, clucks, whistles that stir the mating instincts, warn the young or gather them around the mother to share the food. Every gregarious species depends largely on sounds of this kind, to which the response is highly uniform. But social life in the purely instinctive stage remains necessarily in a rudimentary condition. The hereditary apparatus of itself can do no more than provide certain typical forms of operation, and can neither advance to true parental care for the individual young nor from mere gregariousness ${ }^{1}$ to genuine co-operation. In fact, behaviour testifying to regard for another as an individual is, I believe, confined to the types-mammalia, birds, and possibly the highest insects-among which there is independent evidence of intelligence at the level of that which has been described as the direct correlation of articulate experience.

1 Gregariousness proper is the mere tendency to consort, a tendency which has certain obvious advantages, e.g. warmth, the improved chance of sharing any find made by one individual, and, indirectly it may be, defence. 


\section{Inarticulate Correlation. Feeling.}

It would be difficult to show that the social impulses undergo any substantial change in the lowest grade of intelligence, but there is one point to be remarked. In accordance with our general hypothesis it is at this stage that experienced feeling acquires decisive importance as a cause of subsequent action. It is under the influence of the attendant pleasure or pain that we suppose various modes of action to be built up, maintained, modified or annulled, and if this is so, feeling must become the pivotal point of behaviour. Indeed, instinctive acts also, so far as they are clearly distinct from the quasi-mechanical reflex, must be attended by satisfaction in all that prospers and forwards them, and by pain and distress in all that thwarts them, and there must, accordingly, from the first, be a broad correlation between the pleasurable and the lifegiving, the painful and the unhealthy. It is probable that among the lower animals this correlation is closer than among ourselves. With us, two sources of discrepancy

$X$ arise. (I) While the satisfaction of the organic cravings is generally pleasurable and failure to satisfy them painful, these cravings in the individual may be opposed to the higher functions which membership of the social organism or the mere energising of mental and spiritual activities may impose. In this case, the satisfaction of the organic impulse is a source of pain through the thwarting of another side of our nature. (2) What is a matter of greater difficulty at this stage is the existence of organic cravings which are intrinsically unhealthy, e.g. gluttony, alcoholism, etc. In general, these represent a hypertrophy of a normal impulse which is healthy enough, furthered by the reflective desire for the pleasurable excitement of stimulation, belonging to a higher stage of development. Man not being dependent merely upon instinct and being in some measure master of his life-conditions can, within limits, play fast and loose with himself without undergoing nature's penalty of extinction, and the existence of individuals with exaggerated, deficient or perverted impulses does not involve the destruction of the species. Pain itself 
as a source of nerve-excitement may come to be an object of desire, and it is probable that the physical foundation of cruelty is the excitement of a perverted form of sympathy which the sight of another's pain produces. The mob that used to crowd to an execution and that still devours the newspaper accounts of a murder or gluts itself with details of the chase of a criminal, feels the thrill of the situation without the overwhelming physical or mental anguish which in direct personal suffering soon comes in to quell the hypertrophied lust of excitement. The interest that so many people take in punishment, and that they attribute to the fine development of their healthy moral indignation, is more accurately to be referred to an unconscious lust of a wholly morbid character-the perverted desire for an excitement which the suffering of others affords. ${ }^{1}$

Once again, then, we see how the rough and ready methods by which instinct is correlated with actual requirement, account, on the one hand, for the broad adaptation of organic pleasure and pain to the needs of health, and on the other, for the discrepancies which make morbid feeling possible and allow it to play its sinister part in human life.

\section{Articulate Correlation. Purpose.}

The impulsive act may spring from a feeling but is not directed to an end. Such direction becomes possible in proportion as the present experience becomes capable of suggesting an idea of that which is to come-an anticipation. Such an anticipation charged with feeling is a Desire (or Aversion if the feeling be of the opposite sign), and the action so determined is a purposive act, the content of the idea being the Purpose. With the formation of Purpose we cross the bridge which leads from the action of blind (though felt and conscious) impulse and enter the kingdom of Intelligence proper, and though the basis of the feeling which underlies the Purpose may be wholly instinctive, yet the purposive act will be justly ascribed to the

${ }^{1}$ In detail these excitements depend for their satisfaction on much higher developments than those at present under consideration. But the point is that they have a basis in feeling of a morbid kind, whether congenital or acquired. 
conscious intelligence of the individual. It is correlated with its end, causes and is caused by it.

Just so far as it is intelligent the purposive act may also have ethical value. He who acts with a purpose 'knows what he is about,' and this is the first condition of praise or blame. At this point there are certain incidental confusions against which we should guard. In saying that a man or an animal 'knows what it is about' in doing this or that, we must be careful to understand what sort of knowledge we impute. To do this act $A$ with this end $B$ in view is to have a clear idea of $B$ as a consequence of $A$. It is not necessarily to appreciate all the implications of the act. In particular, it does not imply the conscious application of a general principle, still less of any system of conduct. When a bird procures food for its young or a dog flies to the defence of his friend, we can justly praise the act because it is done with a purpose conforming to our standard of what is praiseworthy. We need not withhold our praise because we deny to the animal any apprehension of that standard as such. It is sufficient that it purposes the individual result of its individual act. But it may be asked, can we not at this rate go a step lower down and praise blind impulse too if it works out to effects which we hold good? The answer is that at the level of impulse the suggestions of praise and blame have no effect, and methods of punishment, if they effect anything, do so not by suggestion, ${ }^{1}$ but by the quasi-mechanical influence of repeated experiences of pleasure and pain. For, where ideas of that which is not yet actual can be attached to the

${ }^{1}$ Conversely, the chiding tone that checks a dog's impulse in full career operates through the suggestion of consequences, and a dog may be seen wavering between the two ends or seeking to carry out his congenital impulse while yet avoiding the results of his master's displeasure. It is of course conceivable that in any individual instance a tone or gesture should have acquired by assimilation direct inhibitory effect without suggesting consequences. Whether this explanation can in fact be applied to the successful and many-sided discipline of the higher domestic animals runs back into the question discussed above (Chap. V. p. 77). We are concerned here with the discrimination of stages as such, and our point is that true praise and blame conceived in their most elementary orm as suggestive of reward or punishment operate through ideas, and are therefore appropriate only when ideas can influence action. 
present, not only one, but two or more alternative ideas are possible. Choice between them arises, and praise and blame, suggestions of reward and punishment, can weight choice by charging one of two ideas with new elements of feeling. The domestic animals are in their degree susceptible to stimuli of this kind, and the way in which a sensible master treats them has its theoretic as well as its practical justification. In sum, with the emergence of ideasthough they be only ideas of immediate ends directly conjoined with present experience and serving as the term of some course of action arising out of such experience-there arise Desire, the conflict of desires, Choice, Purpose, and a function, and therefore a meaning, for the application of praise and blame-in a word, the elements of an ethical order.

Assuming these conditions and no others, we have an order limited to the particular desires of the individual. In the absence of a higher being distributing praise and blame in accordance with a general rule, we have no instrument for the control of present desire, no guide as between conflicting desires standing above the needs of the moment or the wants of the individual, and so correlating present action with the requirements of life as a whole. We are dealing with individual feeling, and the main lines of such feeling are fixed by heredity. On the other hand, the sphere of experience is by this time considerably extended. Experience of results is more rapidly acquired and more freely applied. It can discover new sources of pleasure and pain and induce response to any regular training. We may suppose that the retriever experiences a satisfaction as real in bringing a dead bird to his master as he would in eating it up himself. Further, the more vivid and articulate character of experience builds up a true knowledge of the individuals by whom the agent is surrounded, and with knowledge, the instinctive impulses and feelings of affection, dislike, resentment, jealousy become focussed on individuals. The dog has its regular circle of friends towards whom its behaviour is graduated with some degree of nicety. One is its master, there are others whom it will follow, others, again, whom it greets with friendly recognition but no more, others whom it tolerates and 
others to whom it is hostile. The impulse-feelings on which social relations rest are, in fact, developed in the course of experience and take a variety of individual and concrete forms. The higher animals, therefore, are not merely gregarious, but are capable of the rudiments of family or social life. Parental care is, in all cases, well developed; whether to this is added the life of the herd depends mainly on the method of feeding, which in some cases gives an advantage to the gregarious type and in others makes it necessary to disperse.

\section{The Moral Law.}

From the present point of view-that of a comparison of successive stages in the organisation of life-the essential difference involved in the introduction of the rational factor is the formation of a traditional standard of conduct. Supposing no change at all in the primitive capacities of impulse-feeling, great things would, nevertheless, follow from the power to state in general terms the effect of an impulse, to give expression to the feelings which it excited in those whom it affected or in the onlooker, to distinguish its immediate from its remoter effects and so on. All this is done in effect as soon as class terms arise under which actions are arranged and to which terms of approval and disapproval are applied. There begins then to be a standard whereby action is judged, and this standard is neither the peculiar work nor the personal property of any single man. It is formed in the medium of language, grows up through the interaction of many minds, is handed on as a social tradition and once constituted brings the weight of an external force to bear on the promptings of individual feeling. In the result, action passes beyond the control of momentary desire. It is shaped by a rule of permanent efficacy and of impersonal character.

We have supposed this process to go forward without the aid of any wholly new feeling. But it may be doubted whether at one point such a feeling was not tacitly postulated in our account. We spoke of the feelings excited by an act in the onlooker, and the feeling of the onlooker is the psychological correlative of the generality, the im- 
personal character of the rule. Now it is quite possible that at a lower stage emotions might be aroused by the sight of suffering inflicted, but it is difficult to conceive that at this stage they would be distinguishable from the resentment inspired by any injury to a beloved object. Dispassionate emotion can only: arise in proportion as the character of an act is distinguishable from the person who does or suffers from it. It is therefore apparently dependent on that measure of analysis which we have seen to underlie the formation of language and general conceptions. But it must also be noted as a new and specific development of feeling without which such conceptions would have no efficacy in ethics. It is, in fact, the basis of the pivotal ethical conception, the conception of Justice, and as the response of feeling to the elements of a rational order, we may speak of it as the rational feeling.

This feeling is sometimes identified with sympathy, and, indeed, they are not unrelated. Sympathy may be defined as the tendency to react to the feeling of another as though it were ane's own. This tendency, in the purely unreflective stage, is determined by a pre-existing affection for the individual. It is extended in proportion as the realisation of the life of others enters clearly into one's own consciousness. With this realisation the feeling of another, though it is but an idea for me, is an idea of an experience charged with feeling, and the fundamental fact of sympathy is that in the absence of a counteracting cause the idea has the feeling-tone of its object. Such a counteracting cause, for example, is an emotional disposition of hatred or envy towards the person affected, which overwhelms the feeling of the object and makes the thought of pain a source of pleasure. In the absence of such a transmuting force, the object of the idea determines its feeling-tone in the mind in which it is formed, and a vivid representation of another's pleasure is pleasurable and that of his suffering painful. ${ }^{1}$

${ }^{1}$ The latter is by far the stronger motive. Sympathy with the pleasure of others is apt to be crossed by a morbid egoism which makes the happiness of others into a magnifying mirror of any cross in our own lot, and conversely, I am afraid we are the more ready to relieve the suffering of another because to do so exalts our own ego. 
The extension of sympathy then is conditioned by the limitation and suppression of counteracting emotions and by the extension of the imaginative realisation of the life of others. As this passes beyond the circle of the immediate objects of affection, sympathy begins to be dispassionate and supplies the humanitarian element in conduct. But as the history of human ethics shows, it is only by slow stages that it spreads from the circle of the kindred and the personal friends to that of the community, and from this again to the wider society, the human race and the sentient creation.

But though sympathy is one root of justice, it is not the only one. Primitive, like developed, justice concerns itself not only with the suffering of the sufferer but with the deed of the doer. It is the deed which is directly upheld or condemned, and the rule by which the verdict is determined is a part of the tradition by which the existing social fabric is maintained. What gives force to this tradition is the necessity of a social order as a condition not merely of the healthy life, but of the bare existence of human beings. In the maintenance of this fundamental condition of life, not one but all the living interests of human beings may be said to be concerned. Now this interdependence of the individual and the community to which he belongs is only realised in full at a late stage of reflection, but like other conditions of evolution it operates upon consciousness long before it becomes an object of consciousness. In the present case it operates through the formation of a social tradition, and we may conceive its operation as analogous to that of the environmental conditions in shaping the growth of an instinct. If we conceive a sentiment growing up which would forbid some course of conduct necessary to the maintenance of a given society or allow a course which would be fatal to it, it results that that society must, as a society, perish, or that a counter sentiment must arise in time to check the dissolution. Thus, the actual sentiments that prevail are roughly correlated with the needs of the social structure, without necessarily any conscious reflection on those needs. The one thought-factor that is indispensable is the universal judgment by which a rule is apprehended and applied. But a 
rule that is to be operative in action and to be sustained as a custom must awaken a response in feeling. Now particular rules will awaken particular sentiments, and, conversely, may be engendered by such sentiments. Among other things, it is easy to see that direct feeling for another individual, and particularly feeling for him based not on his personal relationship, but on his membership of the community, would be one very efficacious sentiment in the formation of such rules. But it would not be the only sentiment in operation. On a much larger scale, customs arise as the result of countless individual interactions of impulse and sentiment, interest and counter-interest, and in each case the rule once formed is supported, without regard to its particular character and effect, by a sentiment attaching to custom as custom and condemning its breach. This sentiment does not necessarily imply any clear appreciation of the social order, but it arises in response to the necessities of that order, just as other feelings arise in response to the necessities of life.

In trying to formulate the minimum psychological difference involved in the formation of general rules, we are thus forced to allow one new element of feeling - the sentiment supporting the rule itself. If all the grounds of this sentiment are set out, they involve the whole relation of the individual to society, the recognition of self and others as alike members of a body with rights and duties determined by that membership, and the admission that the life of such a body rests on the observance of general rules impartially applied. But here as elsewhere, feeling, sentiment, impulse arise first, the forces which engender them work in the background and are not made explicit as grounds of action till a later stage of developed reflection. The sentiment of loyalty to the established rule, the feeling that is shocked by a breach of custom, is the simplest form of the response of the individual to the call of social life. Now the individual can respond to the social order only by introducing elements of order into his own life. And while, once again, the nature of this order, the ideal of character or of duty, and the grounds on which it is based are late products of reflection, the direct feeling for the 
admitted rule is the first expression in consciousness of the forces making for that order within which matches the order without. The formation of such an order involves the correlation of different impulses and desires, restraining one, developing another, modifying a third, and the psychological reaction which supports it when challenged is not so much one feeling or sentiment co-ordinate with others, as an effect or precipitate of the entire mass of impulse-feelings that have been brought into a working synthesis. It implies, that is to say, that unity of feeling which constitutes the normal self, the practical side of which is that central control relating the particular act to the general lines of life which we call Will. For Will is the practical expression of system or relatedness as between different elements in active impulse, as reason is the theoretical expression of system or relatedness in the apprehension of experience, and Will is, accordingly, the response correlative to broad and comprehensive ends or to general principles of action, as desire is the response to particular ends. The psychological evolution then involved in the bare formation of human ethics may be conceived as the growth of a synthesis of the impulsive forces of our nature in response to the requirements of a social life. This organised body of impulses expresses itself in consciousness as the sense of obligation to admitted rules, and in action as the control of aberrant desires by will.

From the general conditions of human ethics we may now proceed to the phases of ethical development.

\section{(I) Custom.}

In Ethical as in mental development generally we come in the ruder forms of life upon traces of a stage in which the distinctively ethical categories are imperfectly formed. In all known human societies, indeed, the simpler social rights and duties are in one way or another supported by customs which have at their back sentiments of an ethical character. Yet in the earlier stages there are many indications that what is distinctively ethical has not detached itself from elements of a different origin and character. 
We may take as the general conditions of an ethical judgment that it is ( $\mathrm{I}$ ) a judgment passed on the purposive acts of responsible individuals or on their character as tending to issue in such acts, and (2) an impartial judgment, which, whether explicitly or not, concerns itself with types of action in so far as they affect the general relations on which society is based, in so far as they affect others, and in so far as they affect the value of an individual as a member of society - a judgment imposing duties or asserting rights. Such elements are, of course, made explicit only at a reflective stage, but if our analysis is just a judgment is ethical which in the concrete conforms to them.

Now if we look at the lower grades of ethics we find that outside the circle of the immediate kinsfolk the primary rights of life, property and respect for sexual relations are generally recognised, but recognised in a peculiar form. A breach of these rights is not precisely wrong. It is rather an occasion for the recognised exercise of vengeance. To take the life, wife or property of one who is not a member of the kin, though he be a member of the same society, is not wrong at this stage if it can be done with impunity. It is simply an act which will stir the resentment of the offended man and of his kin, and so lead to a blood feud. At the lowest stages even vengeance is not regulated nor organised, and it is hardly possible to say that there is any regular method of securing redress. But even where redress by the strong arm and the help of the kinsfolk is well established and recognised, it is clear that such a check on transgression is not of strictly ethical character. The act that injures another and threatens social peace is not condemned by any general rule impartially applied. The ethical judgment fails at its central point. In the same way and at bottom for the same reasons it fails in distinguishing intentional and unintentional action, and the vengeance of the blood feud often falls on the kin collectively or on any member of it in place of the individual wrongdoer. If the whole of primitive ethics were of this description we should have indeed to recognise in known and recorded social systems a stage at which the ethical judgment is not yet formed. In actual societies, 
however, we find in fact other fields of conduct in which a more mature stage has been reached. Almost uniformly we find certain transgressions which are punished by such force as collective society can bring to bear-breaches of the marriage taboo and murder by witchcraft being the two offences that occur most frequently in this connection, and thus form the starting-point of a true criminal law. Generally moreover, though on this point exact information is less readily obtainable, the mutual obligations of the kinsfolk may be regarded as true duties, genuine contents of a categorical imperative. It remains that over a large sphere of life, that in which many of the most elementary rights are conceived, the ethical judgment proper is imperfectly formed. The recognised code does not say 'Thou shalt not kill,' it says 'If thou killest, expect the avenger of blood.'

This, it may be, is largely a matter of defective social organisation. The blood feud may be regarded as a stage in jurisprudence rather than in morals. But in the end ethical ideas work themselves out in the structure of law and custom, and the bond of custom in early society is quite strong enough to be a very real force, even if there is no physical force to back it. The characteristic customs of the blood feud prevail, we may be fairly sure, because on the whole they lie near to prevailing sentiment, and this sentiment, which recognises no obligations at all outside the community, admits them within the community, but outside the kinsfolk, just in the form and to the degree which the custom of regulated collective vengeance expresses.

With this half-formed character of the ethical judgment the early conception of the moral sanction is in full accord. Primitive societies have their own theory of custom. They seek a reason for it in one of two directions. Generally the breach of custom brings a misfortune on the transgressor and those connected with him. Precisely how this misfortune operates it is not always easy to say, but in many cases it is clearly connected with the prevailing ideas of magic. In particular the magic power of the curse is an object of fear that may serve to justify the authority of 
father or elder brother, protect the poor from insult and gain the beggar a dole, keep property sacred from trespass and secure respect for the duly sworn oath. But such a sanction is no moral sanction. It is simply egoistic and prudential. ${ }^{1}$ No doubt it embodies the workings of a real ethical feeling. When a man insults his father, jeers at a beggar or breaks his oath, he experiences an internal revulsion of feeling all the more violent in proportion to the $\ddot{v} \beta \rho$ ss of his initial act. In this mood he is ready to be filled with gloomy apprehensions, and in a condition to believe that any threat pregnant with evil will come true. But though the feeling is ethical the expression of it is prudential and, indeed, selfish, and it is with the expression that we are for the moment concerned. Looking at the expression of the ethical consciousness in the belief in misfortune following automatically on transgression, we may say then that it fails to render the ethical judgment (I) in that it gives an external and prudential reason for conduct which, morally considered, rests on quite other grounds, and (2) in that working automatically it takes no account of the character and psychological conditions, while often it is visited equally on the careless or purely innocent act, and falls vicariously on those connected with the actual agent.

Not only magic but primitive animism has its bearing on early custom. But here again we can distinguish a stage at which the operation of the spiritual world is in full harmony with the law of the blood feud. Poseidon avenges the blinding of the Cyclops in the true spirit of the avenger of blood. The rights and wrongs of the matter are nothing to him. That Polyphemus ate several of the companions of Odysseus and did his best to eat Odysseus himself is of no account. He pursues Odysseus from shore to shore, and blocks up the harbour of the Phaeacians who rescue him. The earlier spirits support their wor-

${ }^{1}$ When the calamity is one that falls on society as a whole, society as a whole protects itself by expelling or destroying the offender, and perhaps his relations with him. It is significant of the nature of early ethics that it is just at this point that the conception of a public wrong as against a private injury is first found. 
shippers, protect their haunts and homes, punish their enemies. They are not impartial, supreme authorities, but simply unseen allies to be invoked, or enemies to be dreaded and repelled. We do not, then, in the lowest stages of religion find an explicit expression of the ethical consciousness, but rather a reflection of precisely those defects which we discovered in primitive law.

Upon the whole, then, if the ethical judgment be defined as one impartially upholding rights or imposing duties on responsible persons, it appears true to say that such a judgment is never wholly absent in any known society, but in many rude societies is in large measure unformed and imperfect. It issues in customs which in large measure are neither fully developed morality nor fully developed law.

\section{(2) Law and Morality.}

Early society emancipates itself from the anarchy of the blood feud principally through the growth of a central authority, which by slow degrees takes to itself the function of maintaining order, repressing aggression and retaliation with the equal firmness of the strong hand. Custom at this stage becomes definite law in the sense that it is formulated and enounced by authority and enforced by the executive power. It becomes 'the command of a Superior,' and at least in ideal it is impartially applied. It may be conceived that the development of an organ of impartial administration will forward the evolution of a corresponding sentiment. But whether political circumstances or improved ethical sentiment take the lead in bringing about the advance there is no difficulty in recognising the ethical equivalent of impartially administered law. It is simply the stage of the common moral sense which maintains a miscellaneous set of rules as binding on all persons concerned, which recognises in various men and women various rights, and enjoins on all a number of duties. Into the why and wherefore of these rights and duties it does not enquire. There they are. They constitute morality, and the breach of them is as such immoral. There is nothing here of the hypothetical character of the 
law of vengeance. Whatever their source, the moral laws have a validity which does not depend on retaliation, and is not confined to the weak. The moral law is now as impartial as the king's law endeavours to be. Yet in the face of temptation the moral law must have something to say. The reasons for conforming to it, at other times neglected, must at length come into the foreground, and at the present stage these are of two kinds. There are the temporal penalties attaching to the breach of public law, and there are spiritual penalties attaching to every breach of the moral law, seen or unseen of men. These spiritual penalties may take the form of misfortune in this life, or of punishment after death, whether by reincarnation in the form of a loathsome animal or by being cast into hell. Their points of agreement and difference from the punishments of magic and animism are equally instructive. Like them they are non-moral in that they base the motives of conduct not on the inherent ethical consequences of action, but on external and prudential considerations. Unlike them they are so far ethical that they are applied in general by the impartial judgment of a just God, and fall accordingly on the offender alone, and on him only so far as his $\sin$ is deliberate and unrepented.

This common-sense morality which underlies all the higher religions and philosophies, then, is closely analogous in its successes and its failures to the thought which we also attribute to common sense. It gets on very well until it is asked for reasons. Its rules are felt as rules of morality, as something to which the conscious intelligent being is bound, the breach of which cannot therefore be visited on anyone but the deliberate offender. They are for the same reason impartial. They may, indeed, be very unequal, but that is a different matter. The rights of A or B may differ widely, but whatever they are $C$ is bound to respect both alike. A may have privileges which $B$ has not, but be his privileges great or small, A, like B, must keep within them. The common-sense moral judgment is in this sense as impartial as it is categorical. These are distinctive features of the ethical judgment, and it is only when we reach the grounds of the judgment that the relapse occurs. The 
absence of thought-out ethical grounds forces us back upon an unethical mechanism of extraneous rewards and punishments.

We have said that this incompletely-ethical view is very persistent. But in all the higher civilisations the content as well as the form of the ethical judgment is greatly modified by the reflective systems of ethico-religious teaching by which it is overlaid. If we would know what sort of ethical order common sense elaborates for itself, we must look back to the early civilisations and to the barbarian ancestors of civilised society. ${ }^{1}$ These codes, of course, differ very greatly in detail. For our purposes it is sufficient to remark that they are founded on and serve to maintain the group-organisation of society, which they carry to greater perfection and further elaboration than the ethics of the first stage. Group-organisation becomes a system of peace and, on the whole, co-operation as between the members of a certain body, combined with indifference and even hostility to those without this body. This organisation dominates both the stages which have been described. But (I) in the simplest forms of society the effective group is that of the kinsfolk, who will stand by one another for purposes of mutual defence. As society advances the relations of different kindreds come under more regular control, generally by the growth of the chieftainship, and though the blood feud is only suppressed by slow steps, there arises gradually a certain order in a society resting on other elements than either the tie of blood or mutual fear. (2) In the simplest societies there is only one great distinction, that between the fellow-member of the community who has equal 'rights' and the outsider who in principle has no rights at all. This simple division disappears as in the growth of society there arise within it distinctions of class and rank and of rights in accordance therewith. The class tends now to form a new sort of group within the wider social group. Within it rights are equal, and the inferior has fewer rights, and perhaps (if he is a captured enemy or bought slave) none at all. All that

${ }^{1}$ Though these are in fact still heavily weighted with the ethics of the blood feud. 
here need be insisted on is that throughout the groupformation dominates ethics and law. Man must be loyal, honourable, just in his dealings with his own. As to others-that is another matter. He must, moreover, be ready to fight for himself and his own - and against all else. There is wheel within wheel, group within group-family, kindred, trade or profession, class or caste, the community as a whole. There arise many groups and many loyalties and many degrees of legitimate enmity. But as a whole the life of common-sense ethics is a life of blended cooperation and hostility, of justice and aggression, of love and hate, of self-surrender and self-assertion. All these elements are written deep in the code of common sense, in the personal character that it admires and the system of law that it supports, and if the origin of this code lies in early times, does it need anything but the bare description of it to show that, however much overlaid and held in check by a higher law, it persists to the highest stage which civilisation has yet reached? The ethical judgment is there, but its meaning is not ascertained, and it is allowed to flout itself through mazes of contradiction.

\section{(3) Idealism and Religion.}

Before logical analysis has displayed the contradictions of common-sense ethics the insight of prophets and seers has penetrated the web, and had sight of a deeper truth. A succession of gifted men, or indeed several schools of such men, working in their different ways in Greece, Palestine, India and China, seize for the first time the nature of certain of the fundamental conditions that underlie the life of the individual and his relations to his fellows. They reach down to the life of the soul and the spiritual order, in which the relation of soul to soul is the unitary fact. In form their teaching for the most part is an exposition not merely of the nature of man, but of the being of God or of the laws of existence. In this respect it is largely determined by the general intellectual level of their time, the prevailing interpretation of nature, scientific or metaphysical. But they have certain things in common, whether they work from a theistic basis, like the Hebrew 
prophet and the Christian Apostle, from a metaphysical conception, like the Buddhist, or with a more directly social interest, like the Confucian. Their mission is to interpret the essential nature of spiritual life, and in carrying it out they may justly be said to cut down to a deeper order of reality underlying the world of common sense, just as the reality of science or metaphysics underlies the world of common perception. From henceforward on the ethicoreligious as on the cognitive side there are two ordersthe order that is natural and the order that is spiritual, the order in which the plain man lives and the order which the higher teaching reveals. ${ }^{1}$ In essentials what they report to us of this order may be put in a very broad way as follows. It is the source of that element in commonsense ethics that makes for harmony and co-operation. The stuff of which it is formed-the tissue of the spiritual world -is Love, and from this tissue is woven an ideal of personal character and, in dependence thereon, of social relationships. Of this ideal the suppression of self, and of all that makes for self-assertion, is the warp, as universal benevolence is the woof. Where God is the centre of the whole design, God Himself, at first, as with the Hebrews, the source of righteousness and authority, becomes, as in Christianity, the concrete expression of Love itself, and the relation of the self to God sums up and includes the relation to all other conscious beings. All the characteristics of group-morality, its virtues of pride and grouppatriotism, its antagonisms, its denial of equal justice fall away. The spiritual order allows no such discord. Its peace and goodwill are for all, and it thus lays the basis of a co-operation and a harmony of all mankind. Lastly, the motive which it propounds is no longer some extraneous consequence, but, whether it be the love of God, the perfection of self, or the power of self-surrender, always some inherent characteristic of the spiritual order.

1 At bottom this holds true even of a purely ethical teaching like that of Confucius, since the life that it postulates makes a demand on human nature, which, though less exacting than that of Buddhism or Christianity, will only be met through a special discipline, and in its fullness only by a gifted character. 
While revealing profound and fundamental truths which may as justly be termed true scientific discoveries as any which physical science can boast, this teaching has its limitations and its liabilities to error. Essentially a matter of insight rather than of reasoning, its truths are partial rather than complete, and where it seeks to cover the whole field of knowledge and action it does so rather by deduction from conceived positions than by the patient reconstruction of reality through the piecemeal interpretation of experience. In the support of its central position, which rapidly becomes crystallised in dogmas, it postulates Faith, and Faith comes to replace Love as the keystone of the arch, and so to distort the whole ethical edifice. Moreover, its appreciation of spiritual truth, being obtained rather by penetrating insight into certain aspects than by the resolute effort of reason to grasp the whole, is partial and one-sided. In particular, in insisting on self-surrender it is apt to ignore the claims of self-development, and in dwelling on Love to pay less attention to justice. In holding before the individual the way to obtain peace with his own soul it has less regard for the collective life of humanity, and has little concern for the possibilities of true social progress upon earth. It tends to foster rather than to overcome the antithesis between the world of the flesh and the world of the spirit, and while confident that the one world only is true and real, has practically to abandon the attempt to incorporate the other within it. In the result it either acquiesces in the division of the spiritual and temporal power, or to maintain the form of supremacy explains away its own fundamental teaching. Its comparative failure in practice is therefore not to be attributed solely to the hardness of heart of the sons of men, but equally to its inherent limitations.

\section{(4) Realism.}

These limitations point to the need for a more fundamental reconstruction. The world of ethical thought and practice, the fabric of social institutions in which thought and practice are crystallised, has to be treated as the world of knowledge is treated. It has to be dug out to its foun- 
dations and built over again. We have to get down to the true ethical meanings, the judgments of value which incorporate themselves in rules of action, in ideals of life and forms of social structure, trace them to their generating conditions, and combine them into an order which lends rational significance to the impulse of life as a whole. Such is the avowed task of ethical philosophy, alike in the Greek and in the modern world.

Abstract reasoning cannot indeed play the same part in this practical reconstruction as in the world of knowledge. There is needed an impulse from the actual craving of souls and bodies left figuratively or literally starved by the deficiencies of the recognised social order. There is needed the sensitiveness of the sympathetic imagination to lay. bare the palpitating fibres hidden and too often bruised and crushed under the weight of the social fabric. Hence, particularly in modern times, we often find the most concrete and insistent statement of the problem not in philosophy but in a social or national movement, or, again, in the literary delineation of life as it really is in contrast to the pictures of life which the unreflective social tradition has built up. The true realism of art and literature-and every creative mind is at its best realistic-may be conceived as dealing with a problem very similar in its essentials to the problem of science. Here, on the one hand, is the web woven by society - the mass of existing institutions, marriage, property, the established religions, the current morality, the recognised ideas and sentiments to which all good men are supposed to subscribe. Conventional art accepts this order in disorder, romantic art idealises it. Realistic art has a higher and more difficult task to perform, and it is no wonder if it seldom yields that completeness of aesthetic satisfaction which comes from the contemplation of a nicely rounded whole. Against this screen of traditionally built sentiment it holds up the real man and woman, it seeks to pierce to the heart of their life, to show them as they truly are, and to display the interaction of those underlying forces with the social tissue in which they find themselves enmeshed. It is true that human forces-forces such as these very men and women 
whom the artist seeks imaginatively to realise-have fashioned this network. But fashioned as it is by man, it by no means covers the whole of human needs or expresses. the full possibilities of human life. It is just the particular compromise of impulse with conditions which the jostling of a myriad of forces happens to have brought about. Not but that wisdom, insight, statesmanship have gone to the making of it. Without these no accommodation were possible at all. But their part has been to make the best adjustment possible with circumstances. They have not yet overcome the conditions or made themselves master of the material in which they work. To put the same point in another way, society, though consisting of conscious and intelligent units, is not thereby constituted a conscious whole. On the contrary, the interaction of the units, each with his own seemingly clear purpose, is ever at work producing situations which no one plans. Not only so, but the mass of custom from which law and ethics take their start grows out of an indefinite number of acts, in each of which the individual was conscious only of his immediate end, and had no concern with the social institution which he was all the time building up. But such an institution once formed becomes for action and, indeed, for thought itself a habit, a fixed groove, a category by which henceforward experience will be judged, by which thought will be directed and action confined. It is not till the fabric of custom has been formed that ideals of life take their rise, and so they come into existence confronted, so to say, not with an unweighted experience which they might dispassionately judge, but with man and society as they have been formed by generations of unconscious growth. Hence at the outset the milieu dominates the ideal itself, even when it is an ideal of revolt. The social fabric, with its strange organic power of adaptability, absorbs the independent thought, sucks it into its tissue, digests it and emerges very slightly modified by that which was going to revolutionise it. The ideal so digested becomes a convention, and in the end little more than a form of words out of which all the blood has been sucked, and it is perhaps serving the best function of which it remains capable when it stimulates the 
realist to his task of holding up life as it is against life as it is decked out with convention and smothered in tradition.

\section{(a) The Social Principle in Ethics.}

The realism of art may thus be said to state the problem of which it is the business of philosophy to find the general solution. To this problem the work of ancient philosophy made two great contributions. In the first place, it found a general solution of the problem of the relation of the individual and society. It arrived (with some qualifications, it is true) at the conception that the antithesis between the social and the personal is fundamentally false, and that the true antithesis is between the higher and fuller self which is social, which needs social relations for its content, its filling, and the lower self, which seeks individual satisfaction. This solution has been in substance taken up into modern thought and compared with the idea of selfhood, which the religions suggest has the great merit of placing the conception of personal development in the foreground and putting self-surrender and negation in its right place as a means to the fuller development of self or others. For the same reasons it has the further merit of bringing out the social side of virtue, and insisting on justice as the pivot of the practical life.

In estimating the value of this contribution we must keep in mind a point which tells both on the credit and the debit side. The Greek thinkers were not working with the developed thought of a spiritual religion before them. Greek philosophy was not an endeavour to take rational account of such a religion and work it into the sum of human experience. On the contrary, for the Greeks such a religion lay not in the past but in the future, and their method of approach to it was mainly through philosophic enquiry. They were working up to the fundamental truths, not working on them as conceptions already familiar. Hence the comparative simplicity of their problem and the relative success and completeness as compared with modern systems of their handling. They grasped a smaller range of experience, and held it therefore with a firmer grip. 
But in the second place, as Greek ethics advanced thinkers became more aware of the relativity of their entire standpoint. It became increasingly clear that the city-state was not the only form of human association nor the last word of development. The necessity of a more absolute standard appeared, such as would appeal to the individual in the absence, or perhaps in defiance, of positive state law or generally accepted custom. The Greeks formulated the conception of such a standard in the conception of Nature as that which is valid everywhere and is unalterable by human agreement, and in the law of Nature they arrived on the side of ethics and jurisprudence at fundamental truths parallel to those of the religious consciousness, and asserted Universalism on a different and more logical ground. But though it formulated the conception of an absolute standard from which the actual constitution of society has departed, the idea of Nature contained ambiguities which, as became apparent when it was revived in modern thought, rendered it a dangerous instrument in the reconstruction of social ethics. What was needed was a systematic exposition of the nature of the judgment of value which, if it was to reorganise society, must lead up to and culminate in a comprehensive conception of the meaning and purpose of human life.

\section{(b) The Reconstruction of Idealism.}

But in the interval between Greek and modern civilisation Christianity had absorbed the energies of Europe, and had saturated the social and ethical domains of thought. The stream of Christian development represented a union of Greek rationalism with Oriental mysticism, but in all but its highest expressions it tended to maintain the separateness of the spiritual and material order, nor at best is it free to criticise its own presuppositions and to revise them in the light of the actual needs of human life. Its function is not to discover how life can best be organised so that humanity can make the most of itself, but assuming that it has possession of that which is best, to see how humanity can be made to conform to its rule. Now the concern of modern rationalism-in this linked closely with 
the Greek-is precisely with human life, its needs and its possibilities, individual and collective. The spiritual truths of the world religions are among the leading data of its problem, but they are truths that have to be disengaged from a historical setting which can no longer maintain itself. Stripped of this historical framework, they are seen as truths concerning the soul of man, the position of man in the world, the relations of man to man, and so the fundamental life of society. But whether they are whole truths or part truths is another question, and one which the religions that taught them could not impartially investigate. So the whole problem of life had to be taken up anew, but it could not be taken up precisely where the ancient thinkers had left it. It had been complicated by the deeper conflicts opened up by the fuller religious experience which the world had lived through. For under the influence of religious idealism moral laws acquired a sanctity and an absoluteness which they never possessed on the common sense plane. They were touched, we may venture to say, with that same breath of the Infinite which all through distinguishes modern from classical modes of thought. To adjust their claims to the actual conditions of social life involved a reconstruction alike of ethics and religion which could only be effected by investing social life itself with the same infinitude of meaning. ${ }^{1}$

The relations of the individual and society no doubt are still the pivot on which controversies turn. But the problem is not merely to reconcile their interests. The individual is now a potential centre of resistance, not necessarily on selfish but on the highest ethical grounds. The claims of conscience on the one hand, the order and welfare of society on

1 A single illustration may suffice. No ancient thinker would have hesitated to sanction infanticide as the solution of a sufficiently acute population problem. To the religious mind this solution is barred by the sanctity of parental love and of the new-born life. Modern rationalism would admit this sanctity as one of the conditions of true human development, but cannot, as the religious spirit may, refuse to consider the problem of reconciling it with other conditions equally sacred. It can find a solution only if it can show how to maintain parental responsibility at full power while joining to it responsibility for parenthood to society. 
the other, contend for his obedience. Even to fulfil his own personality may be as much a man's duty as his right. He has become the subject of more than one allegiance, and in virtue of one or other of these may have claims upon society as legitimate as the claims of society upon him. Indeed, there is a sense in which the personal life is more fundamental than the social. For in the instincts, the needs, the impulses of the personality are implicit all the strands that connect the individual with the whole life of mind, whereas in the actual fabric of society wherein he is called to play his part the requirements of the spiritual order may be very imperfectly met. If in one sense society is clearly greater than the individual, there is another sense in which the individual may stand above society, and any reconciliation of personal and social claims must reckon with this relation. The problem then is so to conceive the heightened claims of personality as to make them not disruptive of the social order but working constituents of social harmony.

In the solution of this problem the question of personal liberty takes the central place. Nor will it be personal liberty alone, but liberty for all the forms of social life or combined efforts which arise spontaneously out of the special relations of men that will need consideration. To put it shortly, the synthesis now required is one which will harmonise not merely individual with social interests, but a many-sided freedom, social and personal, with an orderly and disciplined co-operation. In such a synthesis the idea of Development is the keystone. For the implication of liberty is that the error, the wrong and the discord which it renders possible are the price of truth, character and cooperation. In the end we get nearer to truth by letting error develop its fallacies than by stifling it at birth. From beginning to end we develop character not by sheer coercion, but by self-conquest and the knowledge-or rather the full imaginative realisation - of the meaning of good and evil. We approach assured social co-operation not by compelling obedience, but by winning assent. In fine, those things which we ourselves hold true and right and socially just we know for partial truths which will gain in 
the end by the contest with their rivals in the open. But these considerations have weight only when we conceive the social order as a stage or a process of development, and that a development of a spiritual or rational kind. If it were merely a question of realising immediate good as it appears to us, coercion would always be in place." Liberty has its value only in a far longer game.

(5) Now modern thought, as has been said, is concerned with the idea of liberty from the first, but its implications only emerge by degrees. The general problem of modern ethics is to bring spiritual truth into relation with the actual conditions of the development of humanity. In terms of social philosophy this is to achieve the synthesis of freedom and social co-operation, and in the effort to do so we may distinguish three main phases, the first centring on the conception of Nature, the second on that of Happiness, and the third on that of Development.

The idea of Nature descended from antiquity, but, like all ancient ideas, it changed its concrete meaning with time. Nature meant for the eighteenth century philosopher that which would remain if human institutions were in idea swept away. Into this conception he unwittingly imported his own highly developed ideas of right and justice, and the human individual was conceived as seized of rights and possessed of moral qualities which are in reality the outcome of social history. There was in consequence a blending of truth and falsity in the conception. It was true in so far as it conceived human nature as larger in its potentialities than anything which the social fabric had expressed. It was true in so far as it conceived the individual as subject to a higher court of appeal than the judgment of any given society. It was sound in method in that it stated the social problem not merely as a question of identifying personal interest with public utility, but of reconciling the private conscience with public law. It was wrong in so far as it detached the social from the personal life, set liberty in opposition to order, and treated the rights of individuals as limiting conditions rather than as constituent elements of social co-operation. 
In this respect the Greatest Happiness Principle reversed its teaching. It reduced rights and duties, liberty and authority, alike to the position of means to an end, and it set up in the conception of Pleasure and the mitigation of pain a standard of value which it took to be scientifically measurable without dependence on any current ideas or traditions about right and wrong. Here we come to a definite demand for the thoroughgoing reconstitution of human institutions on the basis of an intelligible theory of value. But the theory itself was open to criticism from two points of view. On the one hand, it supplied no adequate account of the ethical motives which it postulated, and arguing that Happiness alone was desirable, it yet pleaded with the individual to sacrifice his own happiness if necessary for that of the greater number, and could overcome the contradiction only by a supposed development of sympathetic feeling which carried little conviction. On the other side, in taking Happiness apart from the fullness and harmony of life on which it depends, it introduced a certain unreality and a certain narrowness into its ideal. It failed to satisfy the deep-seated conviction that man-not only the individual, but the race-has a function to perform, a part to play in things, and that even if the race as a whole could be happy without performing this function yet something essential would be missed.

This conviction is asserted in the biological conception of the expansion of life, the increasing fullness of vitality as expressing at once the direction in which the organic world moves and the goal at which rational man should aim. Unfortunately this conception, being based on physical laws and not on ethical analysis, is generally presented in a form which fails to differentiate the aims of man from those of the tiger and the wolf. The idea of development has received a more human treatment both at the hands of Idealism and of Positivism. In spite of profound differences we have in both these methods of approach the fundamental conception of the human spirit working towards the fulfilment of its own being, evolving out of its cravings and to meet its necessities the institu- 
tions and creeds of successive societies, but freely modifying them in its advance to match the deeper needs of a fuller and more conscious life. The ethical order is the product of a spiritual principle working in human life. This principle seeks, as the ablest of the Idealistic writers teach, to realise itself. It is not complete here and now, but is something in process, in effort. Operating in every individual it moves to the fulfilment of personality, but operating alike in all individuals the development that it seeks must be self-consistent or harmonious, and it is as the condition of such development that liberty acquires its full, positive and social meaning. Lastly, as fulfilment of effort satisfies, the result if not the direct aim of this development is the general happiness.

Indeed, if happiness be rightly defined as consisting in harmony of life, the divergence from the Utilitarian teaching is less marked than appears at first sight. We shall see in the next Part that the Practical Reason must be defined as an impulse to establish Harmony in the world of Feeling, and that this world comprehends all sentient beings, reducing differences of person to a secondary place. Harmony will be seen to imply a relation of mutual support or furtherance, and to be realised in several relations. There is what we know familiarly as pleasure, a harmony of feeling with the environment. Certain conditions yield pleasure, and the pleasure prompts us to maintain or reinstate such conditions. There is again a harmony of feeling with feeling, and such a harmony, where the environment does not conflict with it, is happiness. Lastly, there is a harmony between our feeling and those of others with whom our lives are in contact. This harmony is a part of the rational order and the basis of any Happiness which can be called general. Accordingly, $(a)$ it is true to describe the ethical end as Universal Happiness. But (b) we do not experience either pleasure or happiness in the abstract. We have pleasure in the exercise of our powers, physical, mental, emotional, or generally in the fullness of life. We have happiness in so far as this exercise is in harmony with itself, so that if there is to be a harmony of feeling running through the world of mind, there must be a corresponding 
harmony of life. General happiness would be possible in lives so lived but in no other, as any other is, so far as it is effective, self-destructive. Happiness, then, has as its content a life of a fullness conditioned by harmony. (c) But harmony, we have remarked, means mutual support or furtherance. Two or more functions or forms of life are in harmony so far as they tend to maintain and further one another. Hence, in so far as feeling harmonises with impulse it tends to strengthen that impulse, and, similarly, all sides of personality are thus increased in scope and intensity so far as they harmonise. At the same time insistence on harmony, that is the practical reason, aims at extirpating whatever it cannot reconcile with a harmonious order. Harmony tends to fullness of life, to complete development of personality, though it also limits this development in any individual by the condition that his activity must be such as to promote the development of others. Thus a harmonious development of man in society forms the one aspect of the ethical ideal as the universal happiness forms the other, the two being related as the content of feeling to feeling itself. Both those who have insisted on happiness and those who have insisted on self-realisation have expressed the truth, though it would seem in each case with too much emphasis on one side. The Harmonious development of Mind is at once the substance of general happiness and the end of rational action.

Thus in modern thought the problem of the relations between the individual and society breaks up into several distinct but related problems. On the one hand there are the rights and duties of the individual giving occasion for internal conflict. On the other hand there is the contrast between the actual social order and the true conditions of social welfare, and this contrast necessarily complicates the resulting problem, which is that of the mutual claims of the individual and society. In general the solution to which modern thought has tended lies in the conception of the ethical order as a realisable harmony of many-sided development. Rights are essentially conditions of development, duties are conditions of harmony, so that both are 
elements in social welfare as fully understood, while the actual fabric of society is a partial or experimental approach to the order required. The realisation of such an order would involve the full development of personal capacity, and such development, when shared in common partnership, is the substance of a noble and happy life. The furtherance of such a life has a claim on man through that element in his nature which we may call, indifferently, rational or spiritual.

(6) The validity of this claim will be briefly examined in Part II. Chapter II. Here we lay stress only on the fact of the clear emergence in modern thought of the conception of the ethical system - and with it religious belief and social institutions are bound up-as the creation of human impulses and as the servant of human needs. Modern ethics does not, as has sometimes been held, render the State subordinate to the individual. What it does is to subordinate the State system, including therewith the entire mass of traditionary regulation of life, to the needs of life itself, but the life that it contemplates is that of all humanity. Just as on the side of cognition so here the fabric of traditional thought grows up uncritically under the stress of social actions and reactions. Religious idealism holds up against this tradition a higher ethical order, but still without reasoned demonstration. The critical stage, beginning with the demand for a standard of value, culminates in the conception of the entire ethical order as emerging historically from the structure of the social mind, and subject rationally to the ascertainable conditions of the mind's development. Here again, as in the spiritual religions, the motive is inherent in the nature of the moral order. But it is more fully impersonal than before, the value of conduct lying not in that which the individual attains for himself, but mainly in his service to the greater whole to which he belongs. But the more ethics is freed from religious dogma as an external authority or sanction the more evident it becomes that the ethical order must itself acquire the full force of a religious appeal. To fill our place, to play our part in the moving life of the world 
with all the ardour, devotion and self-sacrifice that that may entail becomes the supreme religious duty. The sense of that life as something exceedingly simple and very close to us in its essence and yet remote and vast in the sweep of its all-embracing order and movement becomes the content of the religious thought. It is indeed impossible to speak of modern religion with the detachment of the historical spirit, for it is all in the making, and it is rather propounding a question than laying down a solution. But certain points appear distinctive. In the first place, the religious order must make its account with experience. In spite of all efforts to escape, in spite of a hundred abortive flights through loopholes of irrationalism and mysticism, religious thought is in its inner consciousness aware that in the end it must abide by reason or perish. In the last resort accordingly it falls back from mythology, from faith, and from intuition on experience. But that is at once to place the actual spiritual experience of mankind in the foreground of religion. The historical forms become secondary. They are reduced to so many incarnations, each infected with the spirit of its day, of the substance which is just all that is noblest in the life of mind. The problem of religion then comes to be to determine what is noblest, and to ask how it has come to be and what it has in it to be. The old order is inverted. What is good and worthy and worshipful, instead of being derivative from an assumed law of creation, become data from which the meaning of life can be inferred and the content of a religious order filled in. If in an earlier phase the moral law was derived from and based on religion, it would be truer now to say that the moral consciousness is one of the startingpoints and strongholds of religious belief. Whereas aforetime ethics had to conform to religious prescriptions, it would now be widely felt that religious conceptions must conform to ethical requirements as verified in human experience. As a consequence the whole ethico-religious sphere is enlarged. It does not become less personal. Indeed its hold on personality deepens in proportion as it is realised that for each man its value depends on the spontaneous response of his whole nature. But it recog- 
nises social salvation as the greater, and including personal salvation within it, and it finds in justice, or what is right in the relation of man to man, a higher spiritual achievement than any virtue of the soul in which the individual can wrap himself in moral warnith.

Lastly, in proportion as the spiritual order is defined in terms of experience its points of contrast with the order of reality become impossible to ignore. The moral indifference of nature forces itself upon us, and it becomes evident that the real as such is not spiritual nor the creation of anything which is purely spiritual, just, or good in our human sense. Reality then is not spiritual, but the spiritual is an element in Reality. It is moreover, if our account of development is just, an element which grows and gathers strength as it attains unity and clearness of purpose. If this is so, we may say that from a Being or Law from which humanity has woefully turned aside the spiritual becomes a life or a principle which is coming into force through humanity, giving unity and rationality to the toil of human beings and through the life of man to the whole world process that leads up to and supports his life. More briefly the Spiritual may be defined as the moving force in ethical development. As such it is an object of positive knowledge, and the entire stream of orthogenic evolution is the revelation of certain phases of its growth.

Ethico-religious progress is not continuous, but we can recognise the principal steps by which the idea of a spiritual order has been attained, purified, enlarged and brought into relation to ethical experience. Nor is the advance continuous in the domain of ethics proper. But it is untrue to say that there have been no discoveries in the ethical field. On the contrary, there have been four such discoveries of capital importance leading mankind through the stages here distinguished. The first is the establishment of the impartial rule, the foundation of commonsense morality. The second is the establishment of the principle of universalism, the foundation of religious idealism. The third is the social personality (if we may use a modern phrase to express the real centre of the Greek doctrine), which governs the first stage of philosophic ethics. 
The fourth is the idea of Freedom, as the basis alike of personal development and social co-operation, which emerges in the modern reconstruction of ethico-religious idealism. But broader and deeper than any definite 'discovery' is the subtle and penetrative change effected by 'reconstruction' as a whole, which transforms rights and duties from restrictive laws into constituent conditions of the desirable life, and though it leaves Morality the master of man makes it the servant of humanity. These discoveries find their ultimate meaning in the conception of a spiritual order not imposed on humanity from without, but growing up within, and directed, through the control of mechanical conditions and by the development of its own many-sided activities, to the fulfilment of the vital capacities of the race. The development of thought, which renders the mind of the race self-conscious, is completed by the development of will, which renders it self-determining. 


\section{CHAPTER XI}

\section{SOCIAL DEVELOPMENTS}

I. WE have traced the advance of thought by itself. But it does not proceed by itself. Both as cause and effect it is correlated with every side of human activity and experience. As cause, because the advance of thought gives fresh power over the environment, suggests new ideals and formulates new methods of social organisation. As effect, because the basis of thought - even of the ideals in which imagination seems to have the freest range-is in reality experience. The most Utopian dream starts from experience, and in proportion as it recedes from experience becomes shadowy and in the end meaningless. Thought in social affairs is not indeed bound close to the realised and the actual. Rather it is tethered to it by a rope which gives it a certain play but confines it to ineffectual struggles if it seeks to wander too far.

Properly to understand the development of mind then we must attempt a very summary view of the corresponding stages in the growth of human achievement in general, of the social structure, the arts and industry. Unfortunately a summary view of social development is more easily imagined than attained. The extreme complexity of the subject, the bewildering mass of cultural data which with all its wealth leaves gaps and blanks where information is most necessary, the difficulties of interpretation and the absence of admitted standards of comparison combine to make the measurement of social progress an exceedingly difficult task. Let us, however, seek to appreciate the general character of the evidence and the possible method 
of dealing with it. The comparative study of culture has as its data first the life of our own world on its manifold sides, its philosophy, science, literature, religion, its laws and customs, its economic structure, its political system, all that we call Western civilisation. Next, still keeping to the contemporary world, there are the old civilisations of the East, and, representing still older levels of culture, the semi-civilised, barbarous and savage communities whose independent life is fading into the past. Thus in the present alone there is an immense field for comparison, but the comparative study of the present could throw little light on development if we knew nothing of the past. To the investigation of present conditions then we have to add a history which for our ancestors extends over about two thousand years, and traces them to a stage of barbarism broadly analogous with some of the lower social types of our own day; while for our civilisation we have a much longer record, extending back through Greece and Rome to the beginnings of Babylonian and Egyptian civilisation in the fourth millennium before Christ. Lastly, where history ends or where it leaves gaps and faults in its record, we help ourselves as best we can with the indirect evidence of archaeology, and with its aid we trace the story of culture, more dimly, indeed, yet still with sufficient light on certain fundamental points, to an epoch so remote that in comparison the whole span of recorded history becomes short.

What emerges from these data? If history had that full continuity for which some writers have contended the answer ought not to be very difficult. The prehistoric movement would be given us by the combined study of archaeology and of contemporary savages. This would lead us to the dawn of civilisation, and from that time onwards the record itself should inform us. But the matter is not so simple as this. In a certain broad sense human evolution may be one process, as indeed all organic evolution may be one process. But if so, it is a unity made up of a thousand different processes-processes, moreover, which, particularly in their lower stages, are not merely independent but largely antagonistic to one another. Just as each separate organic species evolves on its own 
lines, partly no doubt in occult underlying sympathy with others, but also very largely in overt and strenuous antagonism, so does each human community live its own life, flourish and grow through the internal conditions that make it strong, decay and die as often by internal dissolution as by the weight of external force. True, there is always, or almost always, a contact with other communities which is not merely hostile, and the growth of civilisation in particular is forwarded, one might almost say measured, by the development of such contact and the substitution of the peaceful penetration of culture for the internecine war of barbarism. Human history grows towards a unity, but it is founded on diversity, and down to the present day its growth is still to be traced in numerous independent centres of evolution. Now this independence does rot wholly destroy the continuity of history. We ourselves owe our civilisation not to the barbarians of the Teutonic forest, but to Roman, Greek and Jew from whom they learnt. There is a thread of continuity running through all historic culture, but it is crossed and recrossed by many another thread, and the result is at first sight a tangle rather than a neatly woven tissue of clear pattern.

In this tangle we may find a clue if we can seize some distinctive feature of our own civilisation, the latest in time, and therefore the net result of the whole movement up to the present. One such feature has been already mentioned. It is the development of knowledge, a development which is not, indeed, continuous, but which for a simple reason, which I will refer to later, proceeds on the whole more surely and more regularly than any other collective effort of mankind. With knowledge we may rank the control of physical conditions as its immediate result - so immediate, indeed, as in the absence of written records to be a sufficient measure of the degree of knowledge actually attained by a people. Now if we take knowledge and the material arts as a provisional basis of classification, and with this in our minds survey the field described above, we find a vast range of variation presented by peoples still inhabiting the earth. Without going backward in time we can pass from our own aeroplanes and wire- 
less telegraphy to Australians, Fuegians and Veddas, whose life is almost entirely dependent on the caprices of nature. Between these extremes we can place a series of barbaric and semi-civilised peoples of the present day together with the civilisations of recorded history. Beyond the confines of history we find remains testifying at first to a level commensurate with contemporary barbarism, but descending, as we go still further back into the palaeolithic age, to a level even below that of the rudest living savages.

On this side, then, the general drift of human evolution is sufficiently clear. Yet even on this side it is not a straightforward continuous movement. The material culture of classical antiquity was in large measure destroyed in the fall of the Roman Empire, and it was not till the later Middle Ages that all the lost ground was made good. Nor is it probable that this is the only break which a full investigation would disclose. If we speak, then, of a tendency or a progress towards the growth of knowledge and the increased command of nature we must not think of this as an automatic process, as a 'law' of progress which must inevitably effect itself. It is something dependent on further concurrent conditions which may work against it and arrest it. It does not, so to say, represent a straight line to which the movement of humanity is confined and along which it is always marching. All we can say is that, with whatever halts and back turnings, it is a direction in which humanity, or a large part of it, has actually moved a very considerable distance, and is at present moving with greatly increased velocity.

When we pass to other sides of social life these considerations become still more important. It is rarely, if ever, that we can say of any institution or any order of ideas or of activity that its growth can be traced as a continuous process from its first beginnings to its present form. Normally we find a series of actions and reactions, and must be more than content if we can find in the upshot some definite result indicating a net movement in some distinct direction. Take, for example, the position of women. We conceive of the equality of the sexes and the freedom of women as one of the distinctive ideas of modern times, 
and it is not uncommon to hear the position of women spoken of as one of the tests of general civilisation. If this were so, and if progress were continuous and were something that affected the life of society all round, the inference would be that the study of history would reveal a continuous advance in the position of women from slavery to equality. This view will not stand the most cursory examination of the data. Among the historical peoples the position of women has more than once been far higher in many important respects than it was in the times of our fathers, and among savages it is by no means uniformly low. It is, in fact, affected by other causes than the general level of culture, and at certain stages the advance of culture has probably affected it injuriously. Take, again, political freedom. It is an ideal towards which the modern world is still striving. It was in large measure realised by Greece and Rome and the mediaeval city. True, if we look deeper we find that freedom for us has a fuller meaning and a larger scope. It is not to be denied that there are essential differences between a modern and an ancient democracy. But in the interval between them it would be true to say that there were periods when the idea of political freedom was dead. By no stretch of imagination could we represent the measure of political freedom to which the modern world has attained as something towards which the art of government has moved by successive steps all pointing in the same direction. The most that we can say in these and countless similar cases is that, when we consider the life of humanity as a whole and compare our own civilisation with the whole series of earlier forms, together with their survivals at the present day, there appears, when all actions and reactions are set against one another, a certain net movement.

Now if we take social life on its many different sides and consider the development of each, it is quite possible that we shall find a broad coincidence in the net movement, along with great variation in the steps by which that net movement is achieved. But for our purpose, which is that of appreciating the actual result of social evolution up to the present time, it is the net movement which is of primary 
importance. Now this movement has already been set forth on the side of thought, and we have already seen a tolerably close correspondence between speculative and practical ways of thinking. What we have now to do is to consider whether the net movement on other sides of social life exhibits any correspondence with this dual development.

2. It will be well to begin with the development of mind on the side of knowledge, and with the order of ideas and activities most closely related thereto. Here, if anywhere, we may expect to find a correspondence, and this should form accordingly the simplest portion of our task.

On the cognitive side the movement which we traced proceeded from the unformed concept to the common-sense order, from this to the abstract construction of a higher reality, and thence again to the critical reconstruction of experience. This movement is mirrored stage by stage in the methods of organising experience and controlling natural conditions.

The first stage is distinguished by the prevalence of Magic; the second by the rise of the trained handicrafts on a purely empirical basis. The third-the development of a conceptual order-gives rise in the effort to grasp reality as a whole to the dialectical method of metaphysics. In the realm of number, of space relations and of astronomy, so far as dependent on number and space relations it evolves genuine sciences, while in chemistry, biology and to some extent in physics it works out into a tissue of mystical systems with a certain backing of empirical matter. Lastly, we have the scientific treatment of nature generally through the combination of experiment, systematic observation, statistical verification and mathematical analysis. ${ }^{1}$

${ }^{1}$ It is not, of course, to be supposed that these stages succeed one another without blending. The beginnings of a practical training must be put back to the first discovery of the arts. The savage who first chipped a flint probably taught his child to do the same, or was imitated by his neighbour. Nor does magic disappear with the rise of skilled handicraft. It may even be considered as prominent though somewhat transfigured in the scientific mysticism of the third stage. Nor do the rule of thumb handicrafts die out. We name each stage by its leading distinctive feature. This remark will apply to all the distinctions to be drawn in the following paragraphs. 
Passing to practical application, we have first a stage in which man is almost entirely dependent on that which nature offers him for his support. His tools and weapons are of flint, bone and wood. If of metal it is of metal in the pure state requiring no smelting. His dwellings are caves or at best wind-breaks and the rudest huts. His clothing is non-existent or composed of skins and furs sewn with bone needles and animal sinews. His food is obtained mainly by hunting and fishing. He has tamed the dog to assist him in hunting, but has no regular live stock. ${ }^{1}$ He has no knowledge of health and disease, but imputes natural death to witchcraft. His powers of counting are small, and though he can draw and carve, he has no writing. At most he may here and there use pictographs to communicate certain information, and perhaps certain signs by way of memoranda. ${ }^{2}$ The only exception to his general dependence on natural supplies is his power to light a fire, the origin of which is still the subject of guesses rather than of any scientific certainty. Such in rough outline is the culture of the lowest hunting races, now becoming extinct, and, so far as the available evidence enables us to judge, of the Palaeolithic Age. Its broad characteristic is the use of the gifts of Nature with the minimum of transformation. The improved implements of the Upper Palaeolithic levels appear to mark the beginnings of more specialised industries, each with its definite rule-of-thumb tradition. The transition to the Neolithic Age is the result of their maturity. Smoothly polished implements come very largely into use. Spinning and weaving become regular arts, the use of earthenware is general, boats are built, and, according to the character of the environment, society becomes either pastoral, and increasingly rich in flocks and herds, or agricultural, and settled in permanent habitations, often-especially if the Joint Family system develops-of considerable size.

1 'Magdalenian' man would seem also to have driven the reindeer (see Sollas, Ancient Hunters, p. 347).

2 Marks of unknown meaning which may have served these purposes are not uncommon among Upper Palaeolithic remains (Ibid. pp. 243, 31 2, etc.). 
Such is the general character of contemporary barbarism so far as it is not influenced by contact with a higher culture, and such appears to have been the condition of the more advanced peoples in the period between the close of the Ice Age and the beginnings of recorded historypeoples who constructed the lake dwellings and who, when they could control masses of labour, erected the megalithic tombs and temples that remain among the wonders of the world. The third stage is marked by the introduction of writing and by the use of metal, and the two improvements together, taking place in the fertile river valleys, or introduced there by immigrants, aid in the formation of settled states of some extent and population. Writing is still ideographic in the earliest Egyptian dynasties, now referred to the middle of the fourth millennium B.c., but in the Babylonian region the Sumerian script had lost its pictorial form before в.C. 3400. Though iron is found in Egyptian tombs of the first dynasty, it did not come into general use for thousands of years. Both the Sumerians and Egyptians are at first copper-using people. Bronze comes later-it is rare before the twelfth dynasty-and the Egyptians do not appear to have used iron habitually till the seventh century. Gold work, hammered wire and soldering are found in the first dynasty tombs, and copper was cast as early as the fourth dynasty. The introduction of metal into Crete is referred to about B.c. 2800 , and iron appears from B.c. 1200. Glazed pottery beads are found in the prehistoric Egyptian remains, but glassy matter by itself is not earlier than the eighteenth dynasty. Conjoint irrigation works began in Egypt with the earliest dynasty, and the Bahr Yusuf was in working order for 300 miles in the fourth dynasty. Elaborate systems of drainage have been found in the 'Minoan' palaces of Crete, and on many early Sumerian sites. These discoveries are the more remarkable, as sanitation of the kind appears to have completely died out, and it is said that the Minoan system was not again equalled till the middle of the nineteenth century A.D. Oxen were used for ploughing in Egypt from early times, but the horse and chariot were introduced by the Hyksos, and men are not depicted riding until the New Kingdom. 
The shadoof was early in use, but the water wheel does not seem to be verified before the Ptolemaic period. No siege engines are shown in Egyptian monuments, but from the eighth century we see towers raised against besieged cities.

No epoch-making advance was effected in the industrial arts by the Greeks or Romans. Yet Archimedes is credited with the discovery of forty machines, including the compound pulley, the screw pump, and the endless screw, while the Romans had cause to remember his inventions in the art of war. Hero of Alexandria was the first man of eminence who systematically applied science to invention ; but though he made the first steam engine, was familiar with the expansion of air by heat, and constructed mechanisms in which motion was produced thereby, his contrivances were rather scientific toys than machines of practical utility, and this side of his work was sterile till it was taken up again in the Renaissance. Meanwhile the mariner's compass was introduced into Europe from the East - it was known to the Chinese at least as early as the second century B.C. - and the possession of this guide made long sea voyages possible, and led to the discovery of the new world. Chemistry, originating as a practical art in Egypt, had elaborated methods of experiment-e.g. distillation as a means of separating substances was known from the fourth or fifth century A.D. Lastly, to mention only one more point, clocks known to the Greeks in the shape of the clepsydra and the sandglass had developed into weight-driven machines needing only the experiments of Huyghens with the pendulum to perfect them as time measurers. Thus on the one side the more apparent natural forces have been brought into the service of man, wind and water are used to drive mills, animal strength is freely utilised, rivers are canalised, land is irrigated, and fertility maintained by some rotation of crops. On the other hand, many artificial substances are empirically discovered and brought into use- from bronze to glass, and from iron to the elixirs and potions of the older pharmacopoeia. All the elementary machines, the wheel, pulley, lever and screw are in use.

Such, roughly, is the state of man's control over nature 
in the later Middle Age. But in the middle of the thirteenth century began a series of capital inventions which prepared the way for a new epoch. Gunpowder (thirteenth century) transforms the art of war. Printing from movable blocks (fifteenth century) revolutionises knowledge. The discovery of the microscope and telescope (early seventeenth century) opens up new worlds. The barometer (seventeenth century) and the thermometer (I 700) lay the foundations of accurate measurement. There follows on the industrial side the discovery of the smelting of iron with coal (eighteenth century), and then comes the steam engine and the great series of textile inventions which created Lancashire and revolutionised England. These inventions introduce us to a fourth stage in the relations of man to nature.

The discovery of the microscope and telescope reveal new worlds, the development of mathematics a new instrument, the systematic interrogation of experience a new basis. We get below the surface properties of matter, and appreciate and utilise the energies which they conceal. Without seeking to determine the question of the ultimate validity of the conceptions of physics, we may fairly assume that they stand for a genuine advance in insight into the real working of things, and that as the microscope gives us genuine knowledge of a world beyond the ken of the senses on which many of the most important events of our lives depend, so similarly the conception of molecular processes expresses a reality of which chemistry and physics make use, and so, further, the ultra-molecular processes to which the most recent science points, represent again, however inadequately, a further stage in the grasp of reality. The characteristic of the industrial stage in which we live is that industry, following science, goes below phenomena and utilises the unseen forces of nature in engineering, chemistry, medicine and hygiene for the purposes of man. Industrially this stage is marked with some historical definiteness as beginning towards the middle of the eighteenth century. Some of the leading inventions which made it possible go back, as has been shown, to the Middle Ages and even to Greek science, but it was not till this 
period that they began to take full effect. In the earlier inventions, again, the wit of the 'practical' man was still the principal agent, but more and more as development has proceeded has this latest industrial phase come to deserve the name of the stage of Applied Science, and of the control of the underlying forces of nature.

We may perhaps best succeed in fixing the leading characteristics of these stages by considering the materials used by man in each, the motor forces employed, and the methods by which food is won. Thus in the first stage the chief implements are adaptations of materials half formed by nature for the use to which they are put-the chipping of flint, the pointing of bones, the scraping, cutting and stitching of skins and so forth. For power man relies on his own right arm, and for food he goes direct to the products of nature. Beyond this he hardly advances before the close of the Palaeolithic period. In the next stage we may take the potter's art as typical. A wholly formless material is shaped by man to his ends, and with the shaping of the clay vessel we may compare the spinning and weaving which transform fabrics into thread and thread into cloth. Animal power is added to man's, and food is obtained by the breeding of animals and the cultivation of the soil-in both cases by using not merely the products of nature but the productive powers of nature. In the third stage the materials are themselves in part artificial, though their discovery is sporadic and empirical. The great apparent forces of nature, wind and water, are brought into use by mechanical appliances, and similar appliances enable human and animal power to be transformed in kind and direction. Agriculture begins to be intensive, natural fertility is increased, its lack even is made good by manures, and natural species are improved by breeding and grafting. In the fourth stage substances may be disintegrated and reconstructed from their elements. Molecular and ultramolecular forces-vapour tension, electrical attraction and repulsion, chemical affinity - are brought within the comprehension and, finally, within the service of man. Chemistry, bacteriology and the science of heredity are being applied to the systematic production of the best forms of plant and 
animal in their most perfect condition, and the synthetic production of foodstuffs is a further result which it is not unreasonable to anticipate. From the use of the overt, massive forces of 'phenomena' we are passing to the control of the underlying and elementary conditions of movement and life.

3. The control of the environment is one of the two great channels through which the influence of Mental Developments affects the entire social structure. The other great channel is that of the ethico-religious outlook. In ethical development we have distinguished the stage of primeval custom, of the moral common sense, of ethical idealism and of realistic humanitarianism. The centre of the development is the idea of humanity in its two meaningsthe humanity which is in each of us, and the humanity which is all of us. In the first two stages this principle is operative but unconscious. In the third it is explicitly affirmed in various forms of idealistic teaching. In the fourth it is correlated with the conditions of development, personal and social. Now religious development is a web of which knowledge and ethics are the warp and the woof. It is the progressive apprehension of the spiritual element in the world. Its leading moments, as here conceived, have been shortly stated or implied in the account of intellectual and ethical development, and the results may be summarised in a few sentences. In its lowest phase the spiritual, while gradually emerging as an idea out of primitive emotions and quasi-instinctive practices, remains as an idea wholly confused with the material, the unintelligent and even the bestial. It is the stage of animism, of stone worship, beast worship, of the binding of spirits by magic incantations and charms, of cajolements and threats intermingled with petitions. In the second stage the spirit stands out as a clearly recognised personality. It is anthropomorphic, human and even superhuman. In the third stage it embodies the ethical and intellectual ideal. Ethically it is the Perfect God, all righteousness, all love, the source and fountain of human ethics. Intellectually it is the Absolute, the Infinite, even the whole of Reality. 
These attributes are, in fact, irreconcilable, but the upshot of a dispassionate criticism of experience is that, though Spirit is not the whole of things nor their unconditioned creator, it is a dynamic force in things, and a force which progressively enlarges its borders. From being the eternal and immutable basis of order, the spiritual becomes the moving impulse towards that highest order, which may be called the harmony of life, and the evolution of humanity is the revelation of certain phases of its growth.

4. With the advance of ethico-religious ideas the development of the imaginative representation of life is closely linked. For in the deeper and more subtle issues of life abstract thought never satisfies, and we approach the concrete truth by flashes of insight, by emotional suggestion, by constructions embodying meanings which it is hard to state in explicit terms. Imagination, like all thought, is in part an expression of experience, in part a construction for which experience supplies material and suggestions. Hence its work at any stage refiects the extent to which and the methods by which at that stage experience is held together, and corresponds accordingly to the movement of thought in general. Thus the lowest grades of art reflect the incoherence of ideas. Its stories, generally centred in some magical or animistic ceremonial which they 'explain,' are rambling and disconnected. Its attempts at the figure are childish. ${ }^{1}$ Conversely, the clear-cut concrete ideas of

1 I speak of the lowest grade of art, not of an art uniformly formed among the lowest grade of men. In general culture the Bushmen rank almost at the bottom of existing or newly extinct peoples. Judged by their implements, the men of the Upper Palaeolithic period rank clearly below the Neolithic. Yet the Bushmen could draw and paint in a lifelike fashion of which races standing far higher are incapable, while some of the animal drawings and carvings of 'Aurignacian' and 'Magdalenian' man have a force and spirit which puts them not only far above any Neolithic achievement, but in the judgment of many above the corresponding achievements of early Oriental art, and even, according to some enthusiasts, on a level with those of the Greeks. It must be admitted that, though some simple scenes. can be made out, most of the 'Aurignacian' drawings are as wholes of very confused and incoherent character, different figures, however life-like in themselves, being thrown, as it were, pell-mell on to the rock, and that the 'Magdalenian' work is only one 
'common sense' are reflected in the life-like bas-reliefs and statues of early Oriental art, and in the sagas and ballad poetry of Scandinavian, Hebrew or early Greek. Here is life as it is, or stories of life as it has been handed down from mother to child and borrowing a certain heroic proportion from the dimness of antiquity. But though there may be infinite beauty of expression, there is not yet idealism in the strict sense of the term. 'The 'constructions' of this stage are either monsters or magnified men. The third, Idealistic or Romantic stage of art, sets up an explicit cult of the beautiful in life, whether in outward form or in action. It deals with the heroic type, the hero, the saint, the perfect knight or the gentle, lovely, beneficent and virtuous lady, and it admits the ugly, which it also idealises, only for the sake of contrast. This is the typical art, of which the Classic and Romantic are only subordinate species. It is as much the art of Sophocles as of Scott. It is the imaginative expression of the "conceptual reconstruction,' the stage in which the mind moves freely and happily in an order of its own creation. Beyond it, again, lies the art of Realism, which treats the ideal itself as a work of human hands and the Real as something greater than humanity, by which all things are to be judged. In its interpretation of life it has something of the cool detachment of science, and it teaches only by showing how things actually work. It is the art of the Experiential Reconstruction, and as such it regards the ideals of man not as patterns laid up in heaven, but as expressions-imperfect but not necessarily unworthy or unfruitful of human effort and human hopes. In its criticism it uses satire, and sometimes falls into cynicism. But cynicism is not the truth but the failure of Realism, which in essence is founded on a sympathy with the life of man that is wide enough to love the kind for its weaknesses. Of such realism it is

step further on in this respect. It remains that at what is to all appearance a very low general level of development there has in certain cases arisen a sense of line and form, together with a power of execution which altogether disappear at a higher stage. (Cf. Mr. Sollas's Ancient Hunters, for many reproductions of Aurignacian art, Chap. VIII.; for comparison with the Bushmen, Chap. IX.; and for Magdalenian art, Chap. XI.) 
perhaps fair to consider Euripides as the founder, but its wider development is modern, and it is the requirement of realism which has made the novel the dominant form of modern literature, as that which gives readiest scope for the display of the working forces of life in their full extent.

But as a pendant to the realistic interpretation of experience, the mind needs a free range into the beyond, and in proportion as it becomes conscious of the fact that in this range it is transcending the limits of actual knowledge, it needs a vehicle for the expression of those feelings which cannot be formulated without falsity, but which as feelings are driving and impelling forces. It must find a voice for the pathos of limitation and frustration and withal of persistent underlying hope, for 'infinite passion and the pain of finite hearts that yearn.' Such a voice has been found in music. It is to be heard in the modern lyric. The same revolt against human finitude, the same longing for hints and suggestions of a beyond that is known to be unknown inspires the interpretation of nature, whether in poetry or in painting. These, the characteristic modern arts, are not themselves realistic, but constitute those methods of transcending experience which realism sanctions. We may therefore take the critical attitude towards ideals which the term conveys as the characteristic of the most advanced phase of art.

The development of artistic representation does not imply advance in the power to make beautiful things. Beauty is something complete in itself and insusceptible of progress. Everyone would admit that there are passages of the Iliad and verses in Genesis which are perfect, and where there is perfection there can be no progress. On the contrary, the perfect may be a cause of decay since it inspires second-hand imitation, and, generally speaking, an art languishes when that which it has to render has been expressed as well as it can be expressed, until a new genius or a fresh experience opens up a new line. It is probably from this cause rather than from fluctuations in the supply of natural ability that the fortunes of art fluctuate so strangely. The creator is a miner in unknown depths. 
When he strikes a vein he not only gets a rich return for himself, but is rapidly followed by others, who crowd to share the spoil. Work goes more and more merrily as hands increase and the wealth of the deposit is explored, but by degrees the best is carried away, the vein peters out and a few nuggets at most remain for the late-comers. All is dull till a new lode is struck, that is to say, till new experiences open out or new methods of interpretation are suggested. Nor does the new art at its best give us greater beauty than the old, but a fuller interpretation of experience, with a deeper and more truthful expression of feeling.

5. The practical application of the ethical spirit is in the customs, laws and institutions governing the life of society, and we have now to ask how far the net movement on this side corresponds with the development of ideas.

Let us note, first, that the actual efficacy of ideas in the moulding or remoulding of society is itself a matter of gradual evolution. In the lower societies customs change through the pressure of new needs, but that change is unconscious-so much so that if it becomes overt it has to be explained away. This unconscious growth persists in the highest phases, but is almost the only influence of any importance in the earlier stages of social growth. We have next the deliberate acts of a Government, in its simplest embodiment the ordinances of a chief taking particular decisions which affect the welfare of a community or giving interpretations to old customs which substantially make new customs. From this in more developed societies, and particularly in self-governing communities, we pass to deliberate legislation, wherein the community consciously sets itself to remedy inconveniences and redress wrongs, while, finally, legislation comes to rest on more or less systematic effort to secure the triumph of distinct social ideals, and is promoted by voluntary associations directed perhaps to some special end but inspired by a general conception, whether well or ill-grounded, of the true lines of human development.

Thus it is only at an advanced stage that ideas acquire the rational coherence that makes them a force in social life. 
In general the ethical factor is only one of the influences shaping the life of man, and the social structure at any time is the result of the interplay of countless individual forces moved by their own impulses, seeking their own ends, good or bad, social or anti-social. Shaped by these forces, the social structure grows, stagnates or decays. But even when it grows it is by no means to be assumed that it necessarily advances on ethical lines. On the contrary, the mere increment of strength may itself induce elements of discord, and, in fact, of sheer iniquity in the recognised code from which a simpler life is relatively free.

The nature of social growth is best understood by considering the basis of social union, that which tends to hold societies together and also to keep them separate from one another. Certain determinants, such as territorial contiguity or isolation, and community or diversity in ideas, customs and speech are operative at all stages of growth, tending to extend or contract the limits of union as the case may be. But, in addition, there are certain principles which give character to the social structure as a whole, and are distinctive of successive stages of development. The earliest form of social structure is that of the relatively unorganised local group, which takes more distinctly organised shape as a clan, or a community of intermarrying clans. We may reckon these together as societies based on kinship (including affinity). More extensive societies are formed mainly by conquest, and rest ultimately on force. The principle of force clothes itself in the form of authority, and in greater or less degree reacts on the whole structure of society. It may be simply superimposed on the simpler communal life, or it may reorganise it on a feudal basis. It may divide society into castes or into the familiar ranks of nobles, freemen and serfs or slaves. It is the characteristic social form of the middle civilisation. In the higher civilisations it is partially or wholly replaced by the principle of citizenship or mutual obligation as between the community as a whole and its component members. A Society so constituted may be called in the stricter sense a State. It rests on an ethical basis, and is the foundation of ethical development. The State has existed in 
the form of the City State of antiquity and the Middle Ages, and of the Nation State of modern times. But the Nation State in turn is expanded by the federal principle, and federal states and federal or quasi-federal empires begin to foreshadow the possibility of a true international Society. Now in detail this movement does not correspond to the line of ethical advance. The principle of force is the very antithesis of the principle of social ethics. Yet the rise of a higher authority had its advantages as well as its disadvantages. It imposed order and was essential to the beginnings of impartial justice. What is more to our purpose, however, is that the net result, the establishment of the state system in the modern world, provides not, indeed, in its completeness, but in a degree hitherto unapproached the constitutional basis required by the ethical conception of society.

6. Taking the institutions that mainly concern ethics one by one, we find, broadly speaking, analogous phases of evolution. Thus to begin with what lies at the foundation, the method of maintaining social obligations, we have already seen that in the lowest forms of society, apart from certain exceptional cases, scarcely any provision is made. The injured party retaliates if he can, but that is all. As the principle of kinship develops revenge becomes organised, and is the duty of the family or clan. But most ordinary injuries are still matter for vengeance, which pays little regard to circumstances or intentions, rather than for punishment proper. In the authoritarian societies the scope of punishment is extended. The superior maintains impartial justice, and enforces order by severity and even cruelty. On the other hand, it is difficult, and may be impossible, for an inferior to obtain redress from a superior. In the justice of the state the rule of law becomes universal. Differences of rank or position cease to be relevant. But for the maintenance of order reliance is placed less on punishment and more on police efficiency, and in the end on the improvement of social conditions. Crime becomes a disease to be guarded against or cured, and the principles of severity or repression and of purification by suffering 
give place to the idea of doing what is best under the circumstances both for the criminal and for society.

Coming next to the obligations to be maintained, we find the most distinctive feature of development to turn, as we might expect, on the ground upon which rights and duties rest. In the earliest phases corresponding to the two lower stages of ethical development there is a clear line of demarcation between the member of a certain group and the outsider. In the kinship societies there is in general approximate equality between those of the same sex and the same local group or kin, while the 'stranger' stands outside the sphere of obligation. In authoritarian societies differences of rank, caste or class appear, which reflect the same principle of group-morality, though with various modifications of form and limitations of stringency. But in the higher authoritarian societies the ethical principle of equality makes itself felt, and for some purposes at least all Roman citizens under the empire, or again all Moslems and in a measure all Christians are equal, though they may be subject to autocratic rule. Equality, however, in this stage is generally conditional - either on uniformity of religious belief or on some assignable legal qualification. The fuller elaboration of the principle of equality as dependent on the conception of personality is naturally the work of the state, and is carried through in proportion to the thoroughness with which the principle of citizenship is applied. In the modern state the class barrier has disappeared, and the sex barrier is disintegrated. There remains the colour line, of which it is not possible to speak with precision in general terms. In some cases it has been surmounted, in others not. It may be said upon the whole to remain in the modern world the only serious exception to the general rule of fundamental equality of rights and obligations. Lastly, in so far as obligations attach to personality they transcend natural and political boundaries, and this is recognised, both in the higher forms of authoritarian society and of the state, in the growth of international law. The principle that personal rights persist even in a state of war and after conquest is fairly well established. The further development of the same conception which attaches definite 
rights and duties to the state as such and also to nationality as such is, on the other hand, struggling for existence. It is neither a mere ideal nor an admitted principle, but an operative influence contending with immense forces of collective egoism and passion. On the issue of the contest may be said to depend the question whether it is possible to form a true community of the world, and therefore the question whether the work of modern civilisation will endure.

In the history of the family the power of the husband and father has effects which in their way resemble those of the element of force in the social structure. The earlier history of the family-meaning by the term the union of husband, wife and children-is not easily grasped owing to the diversity of types with which anthropology presents us. But alike of the system of mother-right, of polyandry and of the many forms of union in which divorce is so easy that the name of marriage is barely applicable, it would seem true to say generally that they represent the family (in our sense) in an incomplete form. From this point of view the patriarchate is a step in advance. It represents (like the military state as compared with a congeries of illdisciplined tribes) a closer, more compact, more efficient form of organisation. This advance, however, is balanced by the lowered status and unprotected condition of the wife and children, the former of whom probably experiences an actual loss of status in the decay of mother-right. It is accordingly an ethical advance when the rights of wife and children are brought under the full protection of the state. Society in this stage stands in direct relation to the members of the family as individuals, and from this basis it is advancing in our own time to the position of 'overparent,' in which it supervises and at need supplements the functions hitherto left to parental care. This position, it may freely be allowed, raises problems of the relation of parental to communal responsibility which are not yet solved, but it has already developed far enough to enable us to conceive the family as a unit organism contributory to and dependent on the larger organism of the social life. From this point of view then we may distinguish four 
stages - the incomplete natural family; the Patriarchate, the Individualist family, and the Social family.

With the development of the family the whole position of women is intimately bound up. Broadly the development here is a particular case of the generalisation of rights. The superior rights generally claimed by the male are a case of group-morality, and the growing recognition of the equality of status due to woman a simple application of the general ideal of universalism. Historically the case is complicated by the many factors affecting marriage and the family life. Comparing the patriarchate with earlier forms of society, we receive on the whole the impression that women have lost rather than gained status, and there are a few cases in early society when they appear to have an equal or even a superior position to men. Nevertheless, guided by the general and omitting the exceptional, we may regard the position of women in the earlier societies as varying from one of inferior rights to one little distinguished from a servile status. This status is qualified by the social respect which they have enjoyed uniformly in mediaeval and modern Europe, by the ethics of chivalry and the code of the 'gentleman.' A higher stage was reached for a time in Roman law, and has been regained in modern Europe, where it is being rapidly developed to the point of according the woman, married or unmarried, full equality of status, civil, social and political, with the man.

The right of property is recognised in early society in the sense in which other rights are recognised. But the most important property, the land, is more often common to the group. If it is subdivided ownership is hardly distinct from occupation, and when separate property arises it is still rather to the kindred than to the individual that the land belongs. So far private property as a basis of personal enterprise and accumulation hardly exists. With the growth of authoritarian society a feudal tenure of land is generally found in which property is associated with office, rank and status, and is neither in the absolute ownership of the individual nor, therefore, the subject of unfettered bequest or free exchange. With the rise of industry and commerce capital on the large scale 
becomes as important as land, and the tenure of property becomes individualistic, only to give rise, as the economic problem unrolls itself, to new forms of social control. In this case, again, we are at the beginning rather than the end of a stage, and have to recognise a problem rather than a solution. But it would seem clear that the condition of any solution is not to ignore the personal right to acquire, hold and exchange, which has been gradually won, but rather to define more accurately the conditions within which these rights are contributory to the general welfare. The case of contract is closely analogous. Here again early custom reveals contract in our sense as something imperfectly understood. It is not consent but some ceremonial form that is binding, and in the archaic structure of society contract could at best have no important place. The feudal régime admits of contract within limits, but these limits mark out the main lines of life for all classes. Unfettered contract is the ideal of a commercial society which has thrown off feudal bonds, but, like unrestricted individualism in property, is soon seen to necessitate a new form of social control.

With the development of property the ethics of benevolence is closely correlated. Here we have first the simple hospitality of early communism, with as much care for the helpless as the general conditions of life allow. Then we have charity as a duty of the superior, a duty which is also a moral luxury and a means of other-worldly advancement. This gives way to a criticism of benevolence in the interests of individual character, and this criticism, taken in conjunction with the hard facts of economics, is seen to necessitate the establishment of the definite right to the primal needs of a civic life on the basis of a system of mutual obligations as between the individual and the community.

Thus in the development of property and contract, as in that of the family, we notice a double movement. On the one hand, in the more advanced societies there is a breakdown of older social structures limiting the actions of the individual, and so a fuller recognition of personal right. On the other hand there is a process of reconstruction, in which the community as a whole exerts powers and under- 
takes functions previously left to the individual, the family or some other body. This is a fuller recognition of a common collective responsibility. These two elements, personal right and common responsibility for mutual aid, are the two pivotal points of social ethics, and with regard to their relations generally we may say that in the kinship society the individual has little scope for development apart from the common life ; in the authoritarian society his life is usually determined in its main outlines by his status, nor has he any standing ground save that of force for resistance to law and constituted authority. The same is at bottom true of the ancient state, where the subjection of the individual to the common weal is an undisputed axiom. In the modern world there first appeared the conception that the right of the individual as such might limit the law, and this is not merely a conception but a regulative principle in much modern legislation. But it is a principle which has in turn to be checked by the complementary truth that the rights which the individual can claim must be constituent conditions of a self-consistent social order, and to base liberty on law and the common life on liberty is the specific problem of contemporary statesmanship.

Thus, looking through social development as a whole, we observe first the development of an organ of social control and the increasing efficacy of ideas in the organisation of life ; secondly, the equalisation of rights and duties, and the consequent destruction of many of the barriers that divide mankind ; lastly, the development of the principles of personality on the one side, and of collective responsibility on the other. But these are the general conditions of social co-operation, the essence of which lies in the reconciliation of free growth, whether in the individual personality or in the family or in any form of collective life, with organised and disciplined effort for the advancement of the race. Thus, taking each side of law, custom and government in turn, we find that the net movement is to contribute the appropriate condition to the realisation of the ethical ideal.

7. But this net result is arrived at in many cases by very 
devious paths, and of this we can now in a general way appreciate the reason. True social co-operation as here understood involves a reconciliation or synthesis of conditions which in all but their most refined form are opposed to one another. The strength of the blood tie that gives vigour to a barbaric clan, that vitalises the tenderness of natural affection within its limits, maintains a personal and a common pride which is also the source of its warlike prowess. To hate the enemies of the clan is at this stage simply the other side of love for the clan itself. The spontaneous growth of each group means war between the groups. If a higher power imposes peace upon them, there is gain in industry and the ways of peace at the cost perhaps of the vital energy which could only flourish in independence. History is full of such exchanges, in which loss and gain seem almost evenly balanced. To take a single instance. The free Roman Republic had become a corrupt and turbulent oligarchy, wholly incapable of administering the vast dominions it had conquered. The new empire was efficient, and it was equalitarian in tendency. It gave a great part of the world peace and civilised law, and by degrees equality in citizenship. There was great gain here to counterbalance the loss of Roman freedom, and yet we may think that the loss of freedom meant ultimately the loss of life. It is perhaps superfluous to multiply examples. Throughout history an advance in one direction is affected at the cost of loss in another. In particular the growth of Authority, valuable for order, stability, industrial progress and some forms of intellectual development, is often correlated with the most serious ethical retrogression. It is only in the fullness of development that the movement becomes harmonious, because it is only then that it is recognised that the full and true meaning of any form of human activity is to be found in its contribution to the whole, and accordingly not in the repression but in the furtherance of other forms of human endeavour.

Looking now over social development as a whole, we can see a nuch closer correspondence in the net movement than in the stages that make up that movement in each case. 
The fourfold movement of thought pretty clearly reflected in science, ethics and religion is seen also in the direction of artistic creation. It is reflected in the control of physical nature, where the advance is from the empirical handling of surface effects to the apprehension and control of deeper and larger forces. And we now see that in social organisation on its many sides, though the way is crooked, the final tendency is to realise that free co-operation of humanity which is the condition of a harmonious development. This correspondence is no mere parallelism. It is rather the effect of an interaction, and is at every turn part cause and part effect of the then stage of the development of mind. Were society, as some have suggested, really of 'spiritual' character through and through there would be no such interaction. There would be steady growth alone. The parallelism on all sides would be complete. But in tracing the history of mind we are dealing with one cause only-a cause that acts in a milieu of complex forces, but, acting steadily, if our account is correct, gets the upper hand among them little by little.

Lastly, when we seek to conceive social development as a process going forward in time, we must revert to what was said at the outset of the manifold centres from which the movement proceeds. ${ }^{1} \quad$ There are and have been a great number of societies, and their development is in large measure independent and of very unequal rapidity. It is only by a gradual process that civilisation becomes a single stream. We see the process of unification going on rapidly in our own time. In earlier periods interconnection was less constant and less vital, and so, instead of one evolution of culture, there were many evolutions, and certain societies reached a high pitch in one direction or another, even like the Greeks in almost all known directions, which pitch they were unable to maintain. This

${ }^{1}$ One sometimes sees that alleged fact that 'we have not progressed since the days of Euripides' brought forward as evidence that social progress is illusory. As if 'we' and the ancient Athenians were the same people. Certainly there is a moral and intellectual thread of connection. But 'we' are not the Greeks, but Teutons and Celts, and our 'progress' or want of progress since the fifth century в.c. must be measured by what the Celts and Teutons then were, not the Greeks. 
fact alone destroys any attempt to conceive social evolution as from the first a unitary process. Its beginning is with many separate strands, which are but gradually woven together, and this weaving is itself an important part of progress. Or we may think of development as a line along which many societies make independent advances, reaching a certain point and then resting or perhaps turning back. Yet over long periods the result is an advance in the general level, because with the rise of intercommunication one advance on the average helps another, and the highest point of one date becomes the mean point of another.

Into the causes of arrest and decay I shall not here make any general enquiry. It is the bare fact which is important to notice. One thing, however, lies on the surface, and yet is too often ignored. The earlier civilisations were mere islands in the sea of barbarism, and they were liable to constant submersion. In fact in the early history of Egypt, Babylonia and China we come across frequent traces of barbaric incursion, and even where barbarism is overcome in war, the contact with it, as plentiful evidence of our own time shows, tends to lower the standard of civilisation. The Greek state perished in the main no doubt through intestine warfare and the spirit of faction, which were inherent defects of its organisation. But it is also true that it was overwhelmed by semi-barbarous Macedon and afterwards by Rome, whose greatest merit was that she could absorb and apply Greek ideas. It is the fashion to conceive the barbarian conquest of the Roman empire again as a beneficent flood sweeping away a corrupt civilisation. But, in fact, the corruptness of Rome has been greatly exaggerated, and if the Ostrogoths were semicivilised, the crowd of contemporary and later invaders were true barbarians, like the Franks, Lombards and Northmen, or mere destroyers, like the Huns. From the age of Alexander Severus onwards a real process of rebarbarisation began, heralded by the Gothic irruptions of the middle of the third century, arrested by the efforts of a series of vigorous emperors, but destined to go forward till the last of the barbarians were absorbed. This absorption forms a far greater part of history than is as yet understood, 
and when its indirect and subtle effects are compounded with the obvious and immediate will be found to go a long way in explaining the causes of arrest and decay.

That modern civilisation may share the fate of earlier periods of culture is, of course, possible. The reasons for hoping for a better event have been implied in discussing the potentialities of that which we take to be the highest stage of mental development. Modern civilisation stands above that of Greece or Rome not because it has realised greater happiness for the world or a more beautiful order of life or greater works of genius. These things none can measure. Happiness is naught until it is complete, and only full development of Mind could render it secure. If the world process were to be arrested here, it might plausibly be contended that in the actual fruition the life of Athens was something finer and more worth having than the life of England or France. The modern world stands higher because it is further on the road to the goal, though it may be that its portion of the road lies through less smiling country, and it is further on the road because its Thought has advanced a clear stage in the control of the conditions of life and in the conception of its own aim and end. For the same reason it is gradually subduing both A the barbarian without the gate and the Philistine within. 
PART II

THE CONDITIONS OF DEVELOPMENT 


\section{CHAPTER I}

\section{EXPERIENCE AND REALITY}

I. WE have traced the development of mind from the first efforts of adjustment to sense-stimuli in the individual to the point at which the entire collective life is grasped in conception as a unity. We have seen in this conception a focal point upon which the teachings of experience converge, and from which the future life of the race may be controlled. We have traced the advance of the idea of such control from broken, fitful and uncertain beginnings to the same central point of clearness and comprehension. We have shown, finally, that the development is not confined to the world of ideas, but is reflected step by step in the advancing control actually exerted over the physical and social order. But we have not yet enquired into the meaning and the conditions of this development. Our enquiry has been purely historical. We have been content to analyse successive phases and indicate the changes involved in passing from one to the other. We have not sought to determine the future of the development or its ultimate goal, except in so far as this may be implied in some of the terms of our analysis.

We have not even adequately determined the very direction in which development proceeds. For while, for example, we have summarily described the movement of thought, we have not examined the value of the result. We have not, that is, enquired whether we are any nearer to truth at the last than at the first. We have spoken of a critical reconstruction as though it somehow brought us nearer to Reality. We have not asked whether the Mind 
can apprehend Reality at all, and if so, whether it can do so by such methods as we have described. It is clear that our answer to these questions must vitally affect our whole interpretation of the development of Mind, of its drift and tendency. It must also decide our judgment of the relative significance of historical and still more of contemporary movements and controversies. For we are not dealing with a development which is finished, but with one which, however it may have advanced, has left fundamental questions of method still unsettled. As with science and philosophy, so with ethics and social relationships. We have traced the development of the ethical order, but we have not discussed whether the phase which we took to be the latest is also in any justifiable sense to be regarded as the highest. We have not enquired whether its principles admit of any rational justification, and whether, in fine, it can claim any validity which should ground it on something more solid than the fluctuations of feeling and opinion.

But these are the first questions which must be asked if we are to judge of the value and significance, or even of the permanence and probable future of any development of the Mind. A mode of thought, a system of life may be rooted in real conditions which will endure, or it may be forced into existence by some phase of mental climate which will pass and leave it to wither. Which of the two is the case of the evolution here traced? Is it to be regarded as a process of continued approach to Reality, and do the later stages of criticism carry us further forward in that direction, or are we merely substituting one illusion for another, and possibly one that is less pleasing without being less hollow?

Our first enquiry then must be into the validity of the processes of Reconstruction which have been described. We must enquire whether the synthesis of experience gives us knowledge of a real order, and whether the principle of a harmonious development rests on grounds which must be accepted as rational and real. If the answer is in the negative, the movement which has given rise to these conceptions loses all ultimate significance. It is a study in the pathology of the human mind. If it is in the affirmative, a very different position is reached. The develop- 
ment of mind will then be seen as a movement which, after traversing many phases, has arrived at a method of grasping Reality and of directing its own life to ends of real value. In that case the future of development will become a question of the highest interest. There will exist some at least of the conditions of a permanent advance, and it will be necessary to ask what further conditions are required and whether these conditions are realised. This will open up questions of the general conditions of development, and, ultimately, of the whole position of Mind in Reality.

Our first business then is to examine the validity of that Experiential Reconstruction which we have taken as the highest phase in the development of Mind. By a valid process I mean one which, taken as a whole, yields knowledge of Reality. We have to ask then whether any construction of experience can yield knowledge of Reality? May not Reality be not only unknown but unknowable? Or may it be that critical reconstruction, properly interpreted, points rather to some higher way of thinking which puts all ordinary experience in a new light and yields certain fundamental truths which could never be attained by any piecemeal combination of empirical data? Or may it be, again, that it is not by thinking in the ordinary sense but by some form of feeling, intuition or instinct that we approach the deepest truths? All these are questions on which opposite views are still held, views which, if they do not prove the fallibility, at least indicate the incompleteness and immaturity of experiential reconstruction at the present stage of its development.

2. Our theory itself emphasizes this incompleteness, but it implies that, though of course experience does not give us the whole of reality, what it does give us is reality as far as it goes. There is no line of demarcation between that which comes within the sphere of consciousness and that which remains outside. The limits are such as those of the eye and ear, and they are limits capable of being transcended, and, in fact, constantly being transcended as new methods of observation are invented and as new categories or principles emerge clearly into consciousness. 
To justify this assumption would require a complete dissertation on the theory of knowledge, but the heads of argument admit of a rapid summary. In the first place then our knowledge of reality is denied, so far as external reality is concerned, on the basis of an analysis of cognition in general or of perception in particular. The result of this denial is to limit knowledge to a world which the mind makes for itself, whether it be for each the world of his own mind, or whether it be a world in which, in some fashion, all conscious beings have a share. Either view may be combined with an affirmation or with a denial of a further 'real' world which is beyond perception. In the latter case, the theory may be considered not so much as a denial of the knowledge of reality as rather an assertion that all reality exists within the sphere of consciousness. It may be noted, however, that in this view-as appeared at an early stage in the Humian criticism of Berkeleyknowledge of the conscious subject in any sense except that of the knowledge of its passing states is liable to objections similar to those which apply to knowledge of a material order. What has to be said here, however, is that the criticism on which the whole body of these conceptions is founded is an error, traceable to one or other of three main fallacies. The first is that prominent in Berkeley, that in perceiving it is the perception which is our objett. This is, in essence, a confusion between the asserting of a thing and the thing asserted, or between the evidence of a fact and the fact evidenced. The second is that prominent in Kant, that the order which is in the world is not found there by the mind but introduced there by its fundamental forms of sensibility and categories of understanding. This is based in part on an incorrect analysis of immediate apprehension, from which all orderly relations are abstracted, and the remainder is erroneously supposed to be what is actually 'given': in part, on an untenable view of necessity, which is supposed to be an attribute of mental operations instead of being a characteristic discernible in real relations. Thirdly, there is an argument of a more general kind diffused throughout most forms of idealistic writing, that knowledge is relative 
because it involves a relation between subject and object or knower and known. This is a case of the confused transference of thought by which the cognitive relation between the knower $\mathrm{A}$ and the known $\mathrm{B}$ is transferred to $\mathrm{B}$, and because to know is to be in a relation, it is argued that a relation is the only thing known. All that the argument legitimately proves is that $B$ to be known to $A$ comes into that relation to $A$ which we call being known. From such a tautology no human skill can educe a substantial result, either positive or negative. ${ }^{1}$

3. The more serious line of objection to the theory that we know Reality starts from the alleged contradictions of the empirical order. Reality, it is agreed, must be consistent with itself, but experience, it is alleged, contains ineradicable inconsistencies. So far as this is said literally of experience it must be met with a direct denial. Experience can no more contradict itself than can Reality. Contradiction is a relation that occurs between two assertions, one of which affirms while another denies the same thing, and such contradictions arise, not in experience, but in the assertions engendered by thought in the endeavour to interpret experience. Now a thought which contains or involves a contradiction cannot, as it stands, be true. It may contain truth or be partially true, but as containing contradiction it contains error and therefore does not give us final truth. Now the existence of contradictory thinking is a fact with which we are all only too familiar, but fortunately we are also familiar with the compensating fact, that by an extended experience, and, in particular, by a more careful and critical method, contradictions may be surmounted and a deeper or wider view may be obtained, from which both sides of the previous antinomy are seen to contain some truth, while they are in conflict only because they were in some way erroneously conceived. If this is true generally our thought-processes provide the remedy for their own deficiencies, and though our view of reality at any time may involve confusions and mis-

${ }^{1}$ If these arguments appear too summary I must plead that I have set them out at length elsewhere (Theory of Knowledge, Part III.). 
understandings, these would be due not to some inherent defect in thought but to an incompleteness which further efforts might remove. The inference would be not that our knowledge is confined to a world of appearance from which it can never escape, but that it is a knowledge of reality obscured and confused in some degree by limits which it is constantly seeking and often successfully seeking to overstep.

But it is said the contradictions involved in the empirical order are more vital than these. They affect, according to some accounts, the very form of our assertions, and are therefore ineradicable, since in correcting them we make assertions of the same form. The simplest judgment, for example, is said to involve contradictions, and the categories of causality, substance and personality are in the same predicament. These allegations may, I believe, be dispelled by a more adequate criticism of categories. As has been briefly hinted above, when categories like those of identity, of cause or of substance are referred back to the experience out of which they arise and which it is their function to express, the contradictions which appear to beset them in their abstract form disappear. The contradictions supposed to be inherent in thought arise from that ossification which besets thought detached from experience. They belong, not to thought as such, but to a stage of thought, and are overcome by experiential reconstruction. I will not here add anything to the remarks made above (Part I. Ch. VIII.), as the principle of reconstruction will appear sufficiently from the instances given there, and I have discussed many of the chief problems with some fullness on another occasion (Theory of Knowledge, Pt. I. Ch. XII.). But a word must be added on the special difficulties that centre upon the idea of infinity. As to this difficulty we must discriminate. In the bare idea of a space, a time, or a causal process extending without limits there is no contradiction. Contradiction arises, if it arises at all, only when the world of space, time and causation is conceived as a complete system. Now we shall see presently that there is a sense in which the conception of the world as a system is involved in the general postulates of 
thought. This system moreover must be a single system, and it must be possible to say certain things of it universally. These things must hold, however far the system extends, but to assert them is not to enumerate the cases in which they apply nor to define the extent of the system of which they hold. The unity of the system again is not that of a whole defined by limits, but that which consists in the interconnectedness of all causal processes. No knowledge of the ultimate beginning or end of such processes is required. Thought, therefore, does not postulate a closed system. On the other hand, if we ask, not what thought necessitates, but what ideal it sets before us, it would be true to say that it aims at completeness. Now a complete system as ordinarily conceived is incompatible with infinity. For a system must either, according to the well-known argument, be finite. Then it must have boundaries, and there must be something that bounds it, so that it is not the whole. Or it is infinite, and if so it is never complete. Modern mathematical analysis advances a solution by conceiving the infinite as a whole, which differs qualitatively from the finite whole in that it is similar to its parts. Whether the definitions on which this conception rests are free from all ambiguity, and, if so, whether the conception can be fruitfully applied to the world of experience, are questions which I cannot here attempt to determine. But the conception of the infinite as differing qualitatively from the finite emerges also from more familiar mathematical considerations. These considerations lead us to conceive of series which, as they proceed, approximate to a point at which a certain change of character ensues. This point is the limit of the series which it may be conceived as reaching at infinity. Thus the series $.999 \ldots$, which is a fraction, approaches more and more nearly as we prolong it to the number I, which is an integer. The arc of a circle, if we take smaller and smaller segments or remove the centre further and further away, approximates more and more closely to the straight line drawn at a tangent. What is common to these cases which run through the entire world of quantity and are the foundation of the infinitesimal 
calculus, is that a summation of quantitative changes prolonged to infinity amounts to a qualitative change. This result may be resolved into three propositions. (I) No quantitative extension of the series yields the change of quality. (2) Every such extension makes the summed-up series approximate more closely to the different quality, and there is no barrier to the approximation short of the limiting quality itself. (3) If such a series represents successive points in a physical continuum, that continuum may extend up to and beyond the limit without any breach in it.

We have seen above how this conception is applied to the division of the continuous. The point is a part of space which dwindles as division continues. At the limit in which the number of points is infinite its dimensions are also zero. That is, the conception has undergone a qualitative change whereby, instead of conceiving the space as an aggregate of points, we conceive it as a continuum. As we touch the limit we reach a new conception. Now whether the result so exemplified in the case of the infinitely little would have similar application to the infinitely great is a further question. But at least, in expecting that we should find infinite space something qualitatively different from finite space, and eternity something qualitatively different from time, we should be moving in accordance with philosophical tradition.

Before considering this possibility further, let us note the bearing of the discussion on the question of the validity of thought and its relation to reality. Whether we accept the mathematics of the transfinite as philosophy, or merely recall what has been said of the development of the theory of the calculus, we have equally to recognise the transformation of conceptions by contact with the infinite. From this transformation we learn, first, that the discrete treatment of space, time and quantity is inadequate. It does not represent and cannot adequately express continuity of process, of motion, of transition, for when we represent space, time, motion or anything physically continuous by a number, we take it at a certain point, not as in process through that point. But, secondly, a method thus faulty 
in theory could yield results which might be made correct within any assignable limit of error. Thirdly, in vindicating itself against the criticism of its theoretical basis, mathematical analysis advances beyond the discrete treatment, and renders the continuous without error or inaccuracy. Analysis when pushed through corrects its own deficiencies.

These results may be stated generally. A method may be sound for certain purposes though not for others. It may yield a partial appreciation of reality which is just, though it cannot be applied to a final interpretation of reality without contradiction. Thus, methods which enable us to determine that a ball will hit a target, may be vitiated with contradictions if we apply them to interpret the nature of motion. They are founded on certain aspects of motion to the disregard of others. But, secondly, when the flaw is detected, thought is not necessarily helpless. On the contrary, the disclosure of a contradiction is a stimulus to new efforts to overcome it. Thought then at any stage may give us certain facets of reality, and may yet be required to reconstruct its methods in order to deal with other facets, and a fortiori with reality as a whole. It is certain that if we are to grasp space and time as wholes our conception of them must undergo a modification. Without pretending to say in what direction that modification lies, we may revert to an old suggestion in order to illustrate the manner in which it might be effected without destroying the accuracy of our ordinary reasoning. Suppose, in accordance with this image, that space is such that straight lines, simply because they are drawn in space, have an exceedingly minute curvature. It is clear that our calculations, based on the assumption of their straightness, might be accurate within the limits of observable error to indefinite extent. They would only not be absolutely accurate, and only when their inaccuracy became important would serious error arise. Suppose, in corresponding fashion, that time, instead of being uniform, has, in reality, an exceedingly small amount of difference affecting its passage as such. Inferences involving the indifference of time would not be affected unless we were considering 
time as a whole. Such change of conception as these metaphors represent might be necessitated by an attempt to grasp the totality of things, while it would not vitiate the inferences by which we had built up the partial order of actual science.

Now if we had to postulate the possibility of a completed system as the foundation of any inference, the impossibility of rendering it adequately in conception would be fatal. But if such a system is an ideal to which we may approach by repeated reconstructions of thought, no existing difficulty in representing it is an argument against the claim of thought to yield a partial representation of reality. More generally, if there could be no knowledge of reality but that which is final and complete, there could be for us none at all. The whole contention of the experiential method is that knowledge is partial and approximative, and that it advances by constant correction, not only of its results but also of its methoc's and principles. We may know the part without knowing the whole. We may know it approximately without knowing it accurately. Our interpretation of it may be good for the purpose of such partial knowledge and yet liable to final revision in relation to the whole. The methods by which we have arrived at it may be sound methods of dealing with the part, though inadequate to an understanding of the whole of things. Fallacies and contradictions arise when the partial character of knowledge is overlooked. But there is no contradiction contained in experience as such or inherent in the method of interpreting Reality by the correlation of experience.

4. But, it may be contended, the difficulty admitted in conceiving Reality as a whole has a deeper root. It is traceable to the inherent limitations of analysis. But analysis is the condition of rational knowledge, and we must ask ourselves accordingly whether rational knowledge is a tenable ideal. The doctrine that the Real is rational was founded on the presumption that mind is rational. But suppose that mind and all that belongs to it, suppose that life itself is something fundamentally irrational, moving none knows whence or whither as the blasts of impulse carry it. To 
know Reality then, at least to know that part of Reality which most intimately concerns ourselves, would not be to stand mentally at the centre of an articulate system, but to feel in ourselves the impulse or impulses which eddy hither and thither in the vortex. The defects that we have noted in articulate thought would not be defects to be overcome but to be recognised as insuperable. The business of philosophy is to put science under its feet and erect impulse-feeling to the throne. The development of mind is not an extension of rationality, but involves the discovery that rationality plays a humble and subservient part, as an instrument in the hands of the vital impulse.

This theory, however, like most others which decry reason, always uses reason when it can, and in fact seeks to justify itself by evidence drawn from the failures or deficiencies of articulate thought and in particular of analysis. Some of these deficiencies have already been examined. Let us take up one of these cases again and consider how it stands. Life itself, it is said, is a fact with which analysis fails to deal. It cannot be resolved into mechanical forces and therefore cannot be the subject of scientific treatment. There are here two confusions which I believe to be the main ground of the case against rationalism.

That the vital processes must be ultimately of a mechanical character and that they are capable of scientific treatment are in fact two quite different propositions, and the first confusion consists in identifying them. The second proposition, which alone is essential to Rationalism, assumes, no doubt, that they can be clearly and adequately conceived, and it implies that so far as they are complex they can be resolved, by methods familiar to science, into simpler constituent factors. It does not, however, imply - and this is the second confusion - that they contain no element which is unanalysable. On the contrary, it may always be one of the results of analysis to exhibit certain lowest terms as the final products of its work. All that is necessary for accurate knowledge is that these lowest terms should be definite elements clearly presented to the mind. As long as we can justly apprehend their nature, trace the combinations into which they enter and their 
behaviour therein, and record the difference which their presence makes in our world, they are subjects. not merely of knowledge but of the systematic and consecutive investigation which we call science. But, the objector may contend, these unanalysable data, if they are to be the subject of scientific treatment, must be of a mechanical character, and lend themselves to mathematical computation. This is in substance to identify science with mathematics. But for this identification there is no warrant in the postulates of thought. These postulates no doubt lay down that anything that exists must have its place in a system of relations which, when adequately defined, will be found to hold universally. But they say nothing whatever as to the character of those relations, and the conditions of universality and necessity do, in fact, attach as clearly to the means which serve an end, or the functions which together maintain an organic whole, as to the mechanical sequence of cause and effect. The view that Purpose, Value, the whole world of Mind - that which owes its discovery of mechanical laws to its ideal of order-is itself rooted in disorder, is due to an imperfect development of critical method. It may be added that this view becomes a paradox which verges on contradiction when it is suggested that the mind actually implants the order that exists in matter, while remaining in its own nature essentially anarchical.

Analysis then is not destined to resolve everything into terms which can enter into a mathematical equation. Nor does analysis express the entire movement of thought. It may be said to have a direct and an indirect function. Its direct function is to clear up what is obscure and distinguish what is confused. Thus we resolve an ambiguous or cloudy conception into two or more distinct, though allied, conceptions of definite and constant meaning. For instance, a familiar economic analysis resolves 'profits' as popularly conceived into elements of interest, rent, earnings of management and so forth. The work of analysis is here closely parallel to that of careful discriminative attention in the field of sense-perception, which, as we look closely at a picture or long and carefully at a view, 
brings out lights and shadows, outlines, ridges and valleys which go to make up the content of the original perception but are not at first distinctly perceived. So far, analysis merely helps to make the field of consciousness clearer, and it is not suggested that in so doing it disturbs, mutilates or omits. The second function of analysis is indirect. It serves as the basis of comparison, and generally of interconnection. Thus, a piece of country is roughly of triangular shape, and having noted this we are able to apply to it the properties of triangles. Here it is that there is danger of mutilation. The actual surface will not be a perfect plane triangle bounded by three straight lines, but will exhibit irregularities of greater or less importance. In leaving these irregularities out of account, we open a door to error, and it is only by a critical use of the method and the correction of one inference by another that we avoid fallacy. In this usage analysis is the servant of correlation. We break up our concrete, individual experience into elements in order to appreciate the general relations that pervade it. Experience as it comes to us always has its individual character. Even a green or blue colour has in each case, where we see it, its peculiar shade, intensity and quality. But in noting and naming it as green or blue, we assign it a certain place in the colour circle. We note the point in which it resembles all other objects that are green or blue and we are able to predicate of it certain things, as, e.g. that it is at the opposite pole from red or yellow, and to communicate something of its character to anyone who has not seen it. What we say of the object is true though it is not the whole truth, and it is important, because it is the means of bringing the object into relation with objects already known, by subsuming it under an idea which has its place in a system of ideas. Analysis, that is to say, is the basis of the general relations by which we discover system and interconnection running through or, if we prefer to say so, underlying our experience. In the actual process of thought there is, of course, a reciprocal action. Analysis is the basis of comparison and it is also suggested by comparison. We note a certain character in a man's face, perhaps for the first time, when 
we learn that he is the near relation of someone we already know. It is equally possible that we might have been struck by the character and so been led to enquire into the relationship. Our point, however, is simply, that whatever its genesis, the distinct element in the content is the basis of the relations which we discover between different contents. The element which, whether with the aid of much or little or no analysis, whether by much or little abstraction of surroundings, is rendered clear and distinct, is the unit of correlating thought, the basis of the relations which interconnect all elements in the world of experience. Thus, to be clearly conscious of anything is to be in a position to correlate it, to appreciate its relations with any other thing.

Now the impulse to such interconnection is another name for the rational impulse itself. The rationally grounded belief is a belief which is at least seen in connection with others, as issuing from or justified by them. This is the ground of its opposition to the irrational belief, which is so called either because it contradicts others which we still hold, or because it stands alone as an arbitrary dogma which we choose to lay down and do not trouble to prove. But to connect one element of experience with another, we must first distinctly apprehend the elements themselves. The analysed element is the unit of the connected or rational system. And unless analysis is to be an infinite process the ultimate units must be not further analysable. That there should be a limit to analysis then can be no bar to rational reconstruction. It is when we take an imperfect analysis for an exhaustive statement that fallacies arise, and it is probable that the attack on rational method confuses the defective analyses of our actual thinking with the limits that there may be to analysis in the nature of things, and so imputes the fallacies into which we may be betrayed by reasoning from insufficient data to inherent defects of the rational method itself. There is always more in our minds than is brought clearly before consciousness, for, as we have seen, racial experience is acting within the individual mind from the earliest stage, but acting massively so as to produce certain broad resultant effects, 
not articulately so as to correlate relevant point with point. The whole history of the growth of mind as traced by Comparative Psychology, turns on the relation of the conscious life to these underlying forces, and from one point of view the course of development may be said to consist in the steps by which they are brought into consciousness, and that again means in the end the steps by which they are distinguished, analysed and so articulately compared and brought into relation. We have, in fact, seen in the course of our brief sketch how each new stage may be regarded as the coming to light of some factor which was before working in the dark, the rendering explicit of that which was logically implied. The shrinking feeling that is not yet a distinct anticipation of pain, is yet, for the onlooker, a testimony to the pain that has actually been felt and has left its mark. The inference, the practical adaptation of an act to a purpose, for which no logical justification could be given, implies the operation of that which, if it were conscious, would be recognised as a general conception, and the operation of general conceptions rests on rational presuppositions which only the highest stage of reflection brings to the surface. Thus in every stage of conscious development there are at work forces of which an explicit account is given only at the next stage, and as the stage advances these forces become dimly conscious. Darkly and obscurely they rise on the fringe of the lighted area, and their development into explicit ideas is capable of being traced. So in the history of human thought reasons can be found ex post facto for customs and beliefs for which those who held them would give no reason or a wrong one. Magical beliefs incorporate sound social ideas, and the religions teach duties and inspire ideals which are often justified by the reason which rejects the dogmas that first taught them. The working of the unconscious does not cease as the sphere of the rational advances. If the area of our knowledge extends, its line of contact with the unknown is also widened, and we cross the frontier not less often, though with greater caution and perhaps with more fruitful result. The more thought becomes conscious of itself the more clearly it must realise the limited extent of 
the area which it has actually and definitely reclaimed, and the less it can refuse to acknowledge any value in the obscurer and inexplicit promptings of forces that lie beyond its ken. In these circumstances there are three royal roads to fallacy. The first is to regard explicit analysed articulate experience in its existing incompleteness as the sole and sufficient measure of reality, and to dismiss the world of poetry and art, of religious emotion and enthusiasm to a limbo of beautiful imagination. The second is to despise the articulate and abandon the effort to extend its sphere. The third and commonest is to take as articulate truth that which has its foundations essentially in the inarticulate. The feelings which emerge into consciousness clothe themselves in the form which they find at hand. They take up with the body of traditional ideas that lie nearest to them and clothe themselves therewith, not as with a garment but rather as with something that becomes one with themselves. In this process we have already seen the true psychological energy that upholds dogma, and we have seen also that the method of rational criticism is to separate out the feeling from the form which it takes. The mass of impulse and emotion, the body of needs, explicit or obscure, that make up the religious feelings of man, have roots that run deep in our nature. Whatever their source they are as feelings real and vital. We must, at lowest, admit their existence as facts and their importance as forces. We shall, if we are guided by the conception of mental growth as comparative psychology reveals it, go a step further. We shall treat them as indications of a deeper phase of reality which we are only beginning to understand. But we shall also, on the same grounds, resolutely decline to accept as valid the ideas with which they unite themselves. For the explicit idea the logical ground is experience, shaped into thought by processes which can be rendered explicit and justified by rational tests of mutual coherence. Feeling, as such, is no logical or self-consistent support for a belief, and for the extension of our assured knowledge there remains only the one method of the expansion and improved correlation of our experience. This process will, if the source of a feeling lies deep in the realities of our 
nature, of itself bring that source step by step within the circle of knowledge. It will get at the true implication of the deeper experiences as it has reached the roots of those that grow nearer to the surface. Thus, the work of reason appears unsatisfactory, because, at any stage, there is more working in the mind than can get itself clearly expressed. The world of mind is not irrational, but at any stage short of its perfection it is imperfectly rational. The mind at any such stage is more than Reason. Yet Reason is not a separate faculty, dominating one compartment and legitimately excluded from another on which it wrongfully encroaches. Nor does it aim at an aggression which is to domineer or destroy. The weakness or defect of reason is equally the weakness or defect of the non-rational elements. Its extension to them, their inclusion within its sphere, is their redemption. Its legitimate empire is coextensive with Mind, for every feeling, impulse, and even fancy has its legitimate meaning and true development within the harmonious whole towards which it moves. 


\section{CHAPTER II}

\section{THE VALIDITY OF SCIENTIFIC RECONSTRUCTION}

I. So far we have sought to meet the objections which can be brought against the view that rational thought yields genuine knowledge of Reality so far as its sphere extends. If our arguments are sound it may do so. But can we know that it actually does so? If the negative arguments are overcome, what positive arguments can be used? This is to enquire into the grounds of validity, and we must follow this enquiry to the point at which we can apprehend the central conception involved. The question is how a partial experience can be the valid basis of a knowledge which extends indefinitely beyond it. The question becomes the more urgent in proportion as we recognise how narrow are the limits of experience strictly defined. For your experience is not mine, nor mine yours, and in utilising the experience of others we are already committing ourselves to a system of inferences and implications as to the credibility of testimony and so forth, to face which is to realise that any such expression as the experience of the race may be a convenient and compact form of expression, but does not stand for anything that is pure experience denuded of inferential assumptions. If experience is the only trustworthy basis of knowledge, it must be understood that for any one of us it is ultimately his own experience that is meant. But, furthermore, his experience comes to him as a constantly moving stream of change, passing away and partly forgotten as it goes. His knowledge of the past, to say nothing of the future, is at any moment a thought, a judgment that goes beyond the experience of 
the present, and that judgment is liable to err through defect or confusion of memory. The picture that he has even of his own past is not a simple and straightforward reproduction of that which he has actually lived through. Memory is not a cinematograph. It brings together mutually relevant data, it selects and rejects. It analyses and constructs. Pure experience then, in the sense of the sum of the contents present from time to time to consciousness, is only a material on which the mind works, and it is for any one of us a slender material relatively to the wide range of our thought.

What we have to enquire then is by what methods thought treats this material and whether these methods are valid? The broad answer to the first question is that thought acts on its material, ( $\mathrm{r}$ ) by decomposing or analysing it into elements, (2) by bringing different elements together, without being necessarily confined in so doing to the empirical order, (3) by taking the relations which it so finds under certain conditions as true of reality in general, and (4) by comparing its results and correcting them one by another. The broad answer to the second question is that this process of correlation and correction can be so adequately performed as to yield results which, in their general application, will hold true.

2. The central difficulty here turns on the conditions of valid generalisation. We have no a priori guide on the point, for, in fact, simple and uncritical generalisation goes far beyond the limits of certainty. We do not learn to generalise as some have thought. We learn not to generalise as often as we wish. What conditions of generalisation then may be held valid, and why? The difficulty of finding any satisfactory reply to this question has been the persistent stumbling-block in the way of any theory of experiential reconstruction. In particular, it has led both in the theory and in the practice of science to a view which would confine valid reasoning to deduction and allow to experience only the secondary function of corroboration. Reasoning being thought of as essentially deductive in character must be based accordingly on first principles 
which cannot be proved. But it is admitted, that while there are some first principles which are true axioms, needing no proof, there are others which at the outset are mere assumptions, taken up for the purpose of seeing what flows from them. These conclusions can be tested by experience, and if there is agreement, the assumption on which they depend stands uncontradicted. It may be true. If further results are elicited and the agreement with experience continues, it becomes difficult to believe that an assumption which works so well can be false. When it has stood very wide and complicated tests, we need not trouble ourselves to question it further. We may take it as true. This is the only way by which experience can establish a generalisation. Any such generalisation is at first a hypothesis, and in proportion as its consequences are found to conform to fact it becomes a recognised theory.

But though this account is a fair description of what is often the course of discovery, it is in no sense a theory of proof, since it involves the fallacy inherent in the ' inverse' method. If the hypothesis is true, certain observable facts will follow. They do follow, therefore the hypothesis is true. This is inherently bad logic, and the theory that there is no proof obtainable from experience but this is the parent also of much bad science. That discovery should follow this course, that scientific explanation should take this form and that scientific men should shut their eyes to its defects as logical demonstration, are all equally natural results of the position of our experience. We are conscious that it does not, as it stands, yield us the fundamentals of reality, but is an effect or appearance of a more deeply set real order. What, under these circumstances, is more natural than to go outside experience, to make a bold conjectural attempt to seize on some of the fundamentals of the real order, to take up this position as a point of view from which experience will become intelligible, to reason out - as one only can reason from the centre -what effects must follow, and if they coincide with that which we actually find, to rest assured that it is no mere coincidence, but the hand of truth? All this is, we say, natural, but that does not make it less fallacious, it does not 
prevent brilliant hypotheses from acting as mere will-o'-thewisps, nor does it yield a true account of those which have had a more fortunate history.

A brilliant critic of modern scientific hypotheses has summed up the contrast between the historic fortunes of two different classes of theory in the dictum that 'laws of nature are enduring, hypotheses are perishable.' Yet laws of nature are themselves first attained in a tentative way; that is to say, they begin their career in hypothetical form and they often undergo some modification before they set into their permanent shape. What distinguishes them is that though tentatively formulated on the inverse method, they are proved, not by that method but by direct induction; that then (at least in the sphere of physics) they are capable of being put in mathematical form, and that in that form they can be corroborated by correlation with similar generalisations. The hypothesis, which goes beyond that which can be legitimately generalised from experience, has another origin and a different fate. Ordinarily it contains some sound generalisation within it, but at the same time it endeavours to explain this result by means of some concrete image which is intended to reconstruct the reality on which the result depends. Thus, the same critic points out that the old conception of light, as due to material or quasimaterial particles, emitted in straight lines and rebounding from plane or curved surfaces, in accordance with the laws of elasticity, gave a concrete representation of the behaviour of light which embodied, suitably enough, the phenomena in which investigators were then interested, but which, outside the truth which could be accurately generalised from observable data, possessed no validity. The facts of refraction and polarisation necessitated fresh assumptions to make the mechanical model agree with the working experience, but at the same time suggested an alternative image of an etherial medium capable of undulatory movements. This theory was so successful as not only to accord with known facts, but also to give rise to predictions which tallied with subsequent observations, while a crucial experiment yielded results which disposed of the rival hypothesis and coincided with the conclusion deduced from 
the undulatory theory. Nevertheless, logicians like Mill protested from the outset that such coincidence amounted to no proof, and in point of fact, the undulatory theory has given place to a conception of transformations of energy in a medium, the alleged properties of which still present extraordinary difficulties. Professor Ostwald, however, appears to be justified in maintaining that the later tendency is to remove the hypothetical elements and to pare down the theory to a point at which it 'approximates to a correlation of the actual facts destitute of hypothetical elements.' We may believe that the electro-magnetic theory of light has still a long path to tread before this result will be consummated, but Professor Ostwald has well stated the general course of development for theories of the kind. So far as they give rise to new experiments they serve a purpose, but while experience expands, the theory itself is narrowed until the two meet, and what was a hypothetical account of underlying reality - and as such, doubtful and pregnant with fallacy-becomes a descriptive generalisation of the phenomena of the laboratory or of the field, embodied in a series of mathematical equations.

The law of Gravitation, again, is constantly taken as a perfect example of the success of the hypothetical method. Yet Newton himself said, 'hypotheses non fingo,' and his critics have not been sufficiently careful to examine what he meant by this disclaimer. Newton, of course, used hypothesis in the sense that he tried the result of calculating from certain suggested forces, that when the result did not appear to tally with experience, he dismissed it, and that when improved methods of observation showed that it did tally with experience, he accepted it, and regarded his theory as proved. But what exactly was proved? Not the nature of gravitation - as to this Newton had no hypothesis-but the conformity of the earth and planets to the modes of motion discernible in the behaviour of bodies on the earth's surface. The ultimate cause of this action was not only not demonstrated by Newton, but is in fact still to seek. Newton's so-called hypothesis is an extension or generalisation of the motions of bodies from the terrestrial 
to the celestial, an extension at first tentative and then confirmed by the perfection of calculation and observation.

All sound hypothesis, I would venture to say, falls back on this method, though it may not begin with it. Thus, Darwin made a new epoch in biology because he assumed only such causes of variation as were known - the selective action of breeders, as he understood them-and his argument went to show (a) that a partially though not wholly similar selection was at work all through organic life, and (b) that the cumulative action of such selection operating through generations would explain the facts of the organic order. Had Darwin been able to carry through his argument with the precision of Newton, he would equally have proved his theory as a generalised extension to the whole range of organic life of that which can be seen at work in some phases of organic life. The true criticism of Darwin is coming from those who are demonstrating (a) the insufficiency of the kinds of variation and selection of which he had knowledge, and (b) the existence, as a matter of verifiable observation, of other kinds of variation. None the less, Darwin's method was sound because it rested on empirical generalisation. So also is the method of those who rely on experimental breeding or on microscopic examination of the structure and structural changes of the germ cell. The rival method of assuming elements of the germ cell and modes of action within it which cannot be observed, and reasoning therefrom to get back to the facts with as little awkwardness as possible, is one destined to fill many volumes of controversy and to produce theories which undergo transmutation after transmutation before they settle down into accord with the verifiable facts.

3. On the other hand, to limit the work of science to the accurate and compendious description of observable data is to be carried too far in reaction. A theory necessarily aims at something more than the exact description of what it finds. It aims at generalisation, that is, at inference which will enable it to say not only what is found, but what will be found, or would have been found by observation in the past. Such generalisation is secure in proportion 
as it rests on knowledge of causal interconnection, and to know the causes of things is to know their real nature so far as it is relevant. If science contains any adequately grounded generalisations, science is something more than a knowledge of phenomena. But the descriptive or phenomenalist view when pushed through tends to whittle down its laws to abstract equations applicable only to an ideal world, and concerned with the order of perception only if certain elements in that order happen to correspond to the concepts. Indeed, as thus treated they are in danger of being reduced to tautologies. Consider, for instance, the famous equation which lies at the centre of modern physics.

$$
P s=\frac{1}{2} m v^{2} \text {. }
$$

This means that the force $P$ applied to a body to change the direction or velocity of its motion, multiplied by the space $s$, through which its point of application moves, is equal to one half of the mass to which it is applied multiplied by the square of the velocity. Now as a generalisation holding of forces and masses conceived as realities this is a law of the most far-reaching consequences. But its terms are capable of being defined in a manner which brings it perilously near to a tautology. Thus a force, we are told, if by the name we are merely to describe what we can see, is nothing but an expression for a rate of change of motion, i.e. an acceleration. Acceleration is measured by the square of the final velocity divided by twice the space covered in the time during which the acceleration continues. Calling the acceleration $f$, this gives the equation

$$
f=\frac{v^{2}}{2 s} \text { or } f s=\frac{1}{2} v^{2} \text {. }
$$

But the rate of acceleration of different bodies differs, and the measure of this difference is their respective mass, $m$. Bringing this concept into both sides of the equation, we have

$$
m f s=\frac{1}{2} m v^{2},
$$

and writing $P$ for $m f$, we have

$$
P_{s}=\frac{1}{2} m v^{2},
$$

which now means that the achieved acceleration of a body 
in a given space is equal to the acceleration of that body or of any other body which is not susceptible of a different rate of acceleration. If, finally, from this definition, the term body be omitted as not capable of resolution into terms of motion pure and simple, the equation becomes a pure tautology. As such it acquires the appearance of an axiom, but the moment that the question of its truth gives place to that of its application, the fining down of its terms which engenders the axiomatic appearance is forgotten. $P$ becomes an expression for 'forces' taken as real, pressures, strains, stresses, impacts, attractions, and mass becomes an ultimate property of the very matter which the physicist, in his more critical moments, is almost inclined to declare unknowable, while the whole equation, as a result, is a law of the material universe from which the most far-reaching deductions as to the origin and destiny of things can be drawn. In short, in science as in metaphysics there is tendency of ultimate principles to play a double part. To obtain certainty of proof their terms are fined down to a point approximating to tautology, to a point in which, at best, they express the mutual relations of certain concepts. To obtain meaning and width of application the same terms are again expounded to cover the real working of forces that may be but imperfectly seen and known, and are by no means to be controlled by human definitions.

4. What may be called the Hypothetical stage in the development of science moves between two poles of fallacy. In its assumptions about the real nature of things, it goes beyond its warrant, and commits itself to that which its inverse method cannot prove. If to escape this it fines down its concepts to elements which can be educed from experience by analysis, it relapses into a mere construction of a conceptual order with but a casual and uncertain application to reality. So far as it oscillates between the two points of view, it falls into sheer fallacy, and so far as it confines itself to the description of what is given, it abandons the attempt to construe the real order.

At its best the inverse method is an advance on the self- 
criticism of categories, because it requires the systematic test of experience. But to obtain proof we must go a step further, and frankly base our beliefs upon experience itself. But simple as this sounds, and familiar as the method is in the trivial operations of every-day life, to carry it through as a theory of knowledge, and to make experience as a whole the basis of our view of reality as a whole, is the most complex of all tasks, requiring the maximum of selfcriticism in the use of the method, and open at many points to the charge of paradox and self-contradiction. If, indeed, as some of the critics of the hypothetical method have supposed, the object of science were only to describe what we see, the theoretical difficulty would disappear. But if its business is to generalise and infer, be it only to the past and future of our experience, the case is quite altered. Such inference, we have admitted, must be based on a measure of insight into the real causal processes whereby things are determined. But, to assume for the moment that experience gives us reality, how are we to know that it gives us enough of the reality for this purpose? Consider only the relativity of perception. By means of the microscope we know enough now to be sure of the negative truth that the causes of zymotic diseases could not be discovered by any analysis or synthesis of data yielded by the unassisted senses. What reason have we to think that the larger scope afforded by the microscope will carry us any further in the way of ultimate laws? If we rely on observation we never observe the whole of any phenomenon, and there is always the possibility that what is necessary for our purpose resides wholly or in part in the processes which are unobservable. We are brought back, in short, to the initial difficulty, that the world we can touch and see is but a fragment. The results of real processes are visible therein, but we cannot assume that the process as a whole comes within our limits. We may be able conceptually to construct a reality which would yield our results, and this, in fact, is what the inverse method attempts. But to invert the process again and make the results the basis of the construction is a much harder task. It can be fulfilled only if we can answer the two questions 
set out above-under what conditions is generalisation valid, and why under these conditions do we hold it valid? The reply to be satisfactory ought to exhibit the affinity between the generalisations which we use as axioms in mathematics and those which underlie scientific induction. But it must begin with the recognition of their distinction.

5. In fact, the generalisations assumed to be valid in scientific and philosophical thought fall into two classes. (a) On the one hand, as shown in Chapter VIII., we can generalise the results of analysis or synthesis. The function of synthesis consists in grasping distinct contents together. So held they form a whole, and within that whole it may be that characteristics appear, relations of parts for example, which do not appear in the contents taken severally. When we speak of 'appearing' here we are referring, be it remembered, to something which may be a purely ideal process, something that is that becomes part of our mental content when we bring ideas before the mind unassisted, it may be, by any sensory accessories or models. What is assumed by constructive thought and by analysis: also - for the whole of this discussion applies mutatis mutandis to the analytic as much as to the synthetic processis that the attributes there presented by the ideal content will be found in any part of the order of reality which conforms to the elements of which that ideal content is composed. This is to assume that similar elements always constitute similar wholes, and conversely, that similar wholes are always distinguishable into similar elements. The assumption is only true if the whole is nothing but the summation of the elements, and, as we have already seen, it is because in the processes of thought it is so difficult to keep to this condition and so easy to slip in some modification of the elements, that dialectical fallacies arise. What we have here principally to note, however, is that the assumption when most carefully defined is of the nature of a generalisation. It affirms of reality in general a relation which I find true within my mind, or, it may be, in models or diagrams which I can construct. It is, moreover, a generalisation which I assume whenever I put two thought- 
elements together and draw therefrom an inference as to the world beyond my immediate consciousness. To understand why we can make this assumption let us pass to the second class of generalisations. (b) We can generalise any relation of terms if we know that the one holds of, i.e. is related to the other 'as such.' This is the assumption underlying the syllogism, which compels us, if we argue from $A$ to $B$ in a given case, i.e. if we take $A$ as a sufficient ground for the assertion of $B$, to admit the major premiss any $A$ is related to $B$ in the specific manner stated. What is true of a term as such is true of it universally, is the general postulate of critical reasoning. But what does 'as such' mean? Whatever else it may mean, it includes this negative, that the relation which holds between two terms as such is not dependent on any third term. It follows that any given relation of two terms not dependent on a third term is universal, and it results further that if any relation between two terms A-B is given in experience only two alternatives are possible. One is that the relation holds of one or other of the terms as such. The other is that it is dependent on a third term. To distinguish the two cases is the problem of scientific induction. Lastly, it will be seen that another way of stating the same assumption is that every element of experience is related to some other element of experience as such.

If $B$ is true of $A$ as such, $A$ is called the ground and $B$ the consequent. There is nothing in the propositions laid down to show that the same consequent may not have several grounds. Thus A-B may be universal while B-A is not so. But if B-A holds in one case and not in another, it must be because there is a third term $\mathrm{C}$ in the first case which changes to $\mathrm{D}$ in the second. Hence the full consequent of $A$ is not $B$ but $B C$, and the relation $A-(B C)$ is convertible, while some other ground $\mathrm{E}$ of $\mathrm{B}$ has as its full consequent BF. Different grounds, therefore, have as such different consequents, though these different consequents may happen to agree in some point. These assumptions, all thus traceable to a single root, can be shown to apply to the whole of the functions of thought, and to be the sole assumption made in thinking.

(I) They include 
the previous class of generalisations. For any characteristic of a whole, if it really is nothing but a characteristic of that whole precisely, is something true of the whole as such. It is not dependent on any third term, except it be one on which the whole depends, for its existence is a part of the existence of the whole. The same is true of the whole as compared with the several parts which truly constitute it. Hence, lastly, the element which characterises the whole as a whole is true of the parts which constitute the whole as such, and therefore universally. (2) The assumption covers the laws of causation and of the permanence of substance. For the assumption is that a relation holding between two terms as such at any given time in any given part of space will hold at any other time in any other phase. This is to assume that time and space as such are indifferent, and do not affect the relations of contents within them. It follows that if any element A persists momentarily, this persistence may be regarded as a relation $\mathrm{A}-\mathrm{A}$, and if this relation is not due to some external condition it is self-determined, and is therefore indestructible and eternal. It follows, secondly, that all change is a process of continuous becoming which, given any phase in its completeness, will run the same course uniformly. If $A$ is followed by $B$, either A must be a phase of continuous process of becoming which yields $B$ as a subsequent phase, or $\mathrm{B}$ must arise from some other source $\mathrm{C}^{1}$ which must be of that character. Whatever the cause $x$ which yields $\mathrm{B}$, it cannot persist momentarily unchanged and then give place to $\mathrm{B}$, for this would imply that it suffers at one time a change which it does not suffer at another without any difference in the elements of reality to account for it. Hence everything that exists is either a substance, and therefore unchanging, or a phase in a continuous process following immediately on an antecedent phase which passes into it, and which will always wherever it recurs undergo the same transition, or lastly, a phase in a process which is in part self-determining and is indestructible and in part a process of change-i.e. having both substantiality and causality. Furthermore, in any complex process of becoming in which,

${ }^{1}$ It may be other only in the sense that $\mathrm{C}$ is needed to complete $\mathrm{B}$. 
say, $\mathrm{ABC}$ becomes DEF, there may be an element $\mathrm{F}$ common to FGH, a phase in quite a different process LKM. In this case $F$ is said to have two distinct causes, but (a) the causes as wholes have effects which differ as wholes, and (b) each cause is still a phase in a transition which leads inter alia to F. (3) These assumptions explain scientific induction both in its successes and its difficulties and failures. They show that the problem of thought is that of disentangling the relevant from the irrelevant. In experience as it comes to us the two are involved in what at first appears as a hopeless tangle. Continuity yields the first clue. What persists amid change is taken provisionally as self-determined, i.e. as substantial, and the process that goes on steadily and is repeated when circumstances differ is treated as self-determining, i.e. as causal. The principle thus roughly carried out by common sense may be simply formulated thus. Let an element $A$ be introduced into an environment $B C$, and be the starting-point of a process $A-\alpha$. This process is not due to $B C$ as such, because it did not arise till $\mathrm{A}$ was introduced. But $\mathrm{BC}$ may contribute to it. Then let $A$ also be introduced into the environment $D E$ having nothing in common with $\mathrm{BC}$, and let the same result follow. The process $A-\alpha$ is then not conditioned by any part of the environment, that is, it is self-determining.

This statement of the method of scientific induction is open to criticism along two lines. One attacks its form or principle, and founds itself on the Plurality of Causes. B or C, it argues, may be the cause or part cause of $\alpha$ in the one case, $\mathrm{D}$ or $\mathrm{E}$ in the other. But we have seen that the plurality of causes is a doctrine of limited application. BC on the one hand, EF on the other are either permanent, and if so do not yield any element of $\alpha$, or they are phases in a transition, or processes. If either of these processes might be regarded as leading to one of the conditions of $\alpha$, the same cannot be true of the other, for they are ex hypothesi alike in no respect. They neither are nor are becoming alike. If the hypothesis then be granted the universal relation $A-\alpha$ is established.

But at this point more substantial difficulties are opened up. How can so much be known about the concomitants? 
We are always in contact with processes which escape observation. How can we be sure (I) that in any case, even when we appear to control the whole of the conditions, A is the sole change introduced, (2) that the operation of the concomitants $\mathrm{BC}$ or $\mathrm{DE}$ does not consist in inner processes having points of agreement which we cannot detect? There are two possible answers. One is an appeal to the theory of chances. It is against all probabilities that, if we go on varying the circumstances of an experiment, we should always light on specially favourable conditions. The other is an appeal to verification. The generalisations which we make on this basis conform to fact, and calculations derived from them, deductions, constructions, yield results which observation confirms. I will confine my remarks here to the second argument, and see whither it leads us.

That results conform to observation is not in itself any proof of the principles on which the calculation is based. But results may under given conditions be logically used in the corroboration of principles. Suppose that from two causes in conjunction we infer an effect. Then suppose it established by an independent generalisation that those effects actually follow from those causes when conjoined. Let the simple causes be $a$ and $b$, and their effects $c$ and $d$. It is clear that if we take one of the causal relations, say, $a-c$ as certain, and the other one $b-d$ as less certain, the probability of $b-d$ is raised. For if $b-d$ did not hold true, then the generalisation $a b-c d$ could not be true, and of $a b-c d$ we have independent probable evidence. But further, if $a-c$ is itself not quite certain but only probable, still its probability increases that of $b-d$. Again, $a-c$ may be similarly corroborated by a fresh conjunction, as ae-cf, where $e-f$ has an independent probability, and this process may go on indefinitely. In point of fact, any well-developed science does exhibit a network of such interconnected generalisations, and these are the tacit, though seldom expressed, ground of confidence in the general adequacy of the observational methods on which it rests. The confidence in rational method is thus legitimately strengthened by actual success in establishing rational interconnection. 
6. But it may be asked, at what stage does corroboration yield certainty? In any system each constituent generalisation is open to the doubt whether all the relevant circumstances have been taken into account. At what stage and on what grounds can we regard this suggestion as overthrown? To answer these questions we must, I think, recast the form of statement of the principles on which inference works. We have assumed so far that a relation which is independent of any outside terms is universal. This as it stands is an assertion about reality. Suppose we say instead that an 'observed relation of which no external ground is in any way suggested by experience is a logical ground of a universal assertion.' This is not only an assertion about reality-though it is indirectly an assertion about reality-but also a rule for our thought. Let us take this as the principle of our reasoning and see what it leads to. Clearly it would not be a sound rule if the above assertion as to reality were untrue, but that assertion might be true, and yet this principle might be unsound. In assuming it, therefore, as sound, we are assuming something more than before. We are assuming, since it is a rule for guidance, that there is some good to be got by its means. That is not, as I shall presently show, the whole basis of the assumption, but is at least one of its implications which is corroborated if good results.

Assuming, however, that this is the implication of rational thought, we see that any generalisation to which exception can be taken on the ground that experience does suggest the possibility of some external ground is to the extent of the value of that suggestion doubtful. In point of fact, we do hold generalisation in suspense as long as experience does suggest any possibility of the kind. If no specific interference of a disturbing condition is suggested, there still remains a general doubt of the adequacy of human observation based on the fact that with the utmost care we sometimes err. Our ordinary inductions may be regarded as open to a vague doubt of this kind. On the other hand, if we have succeeded in establishing a system of interconnected generalisations of a kind which has never been shaken, of such a system we might say that experience 
does not suggest but repudiates the possibility of error. The value of the doubt, which throughout is grounded on experience, must then fall to zero, and we could speak of certainty.

Of certainty, that is, if we assume the legitimacy of our axiom. But on what does this axiom rest? Not, I think, on the fact that it actually gives results which harmonise, though that is a necessary condition of its validity. If it failed to give harmony it would be contradicted by its results, and must be false. But though its results do not contradict it, this only proves that it may be, not that it must be, true. On what then is its certainty founded? On this, I think. Every act of thought, at any rate every act of inference, may be regarded as an implicit judgment connecting premiss and conclusion. Every such judgment is concrete, having to do with the specific facts before it, but any such judgment has also a certain class character, can be analysed into an abstract assertion. Find that assertion and you have a generalisation which the mass of our inferences support, which if true maintains, if untrue destroys them. We can, in fact, prune off false elements of inference by thus generalising and comparing them with one another. Similarly we find true inferences corroborating one another in that they involve or lead up to a single principle. The principle of reasoning then rests on the multifold separate acts of reasoning, and expresses their harmony with one another.

We are thus led to the conception of knowledge not as a body of thought guaranteed by its dependence on first principles, which stand above all criticism, but as a body of judgments, the strength of which lies in the fact that in the main they support one another. This is the systematic view of knowledge, and we may ask (a) whether it conforms to the requirements of the conception of rational validity, and $(b)$ whether, if so, it throws any clear light on the value of the thought that we possess regarded as an interpretation of reality.

7. Let us first ask what is meant by the term Rational, and in doing so let us start afresh, without assuming any of the points advanced in the preceding argument. 
We may obtain some light on the question by asking, first, what is irrational? Two types readily suggest themselves. There is, first, the inconsistent. It is irrational to maintain contradictory positions. There is, secondly, the arbitrary, and of this we may distinguish two cases. Negatively it is irrational to maintain a position without reason assigned. Positively it is irrational to maintain it on grounds of emotional feeling, because we choose to maintain it, or from any cause proceeding from our own peculiar mental make-up rather than on account of the intrinsic character or relations of the conduct asserted. Both these rules, however, present great difficulties. To the first it may be held that there are at least some exceptions. It is a possible view that there are some self-evident truthstruths, therefore, which may be maintained on no other ground but that of their inherent character, and it may be urged that the bare conception of a 'ground' implies truths of this nature. For let us admit that it is unreasonable to make or maintain any statement or position for which no grounds could be assigned. Then if any proposition is not self-evident, the 'grounds' on which it is asserted must, it would seem, involve something further than anything contained in the original position. This is as much as to say that what is maintained must be somehow connected with what is otherwise known or thought, and that to reason is, in the very broadest sense, to interconnect. But this at once raises the question of the ultimate goal of interconnection. If it be admittedly arbitrary and irrational to advance proposition A without some ground, is it made reasonable when such a ground is discovered in proposition $B$ ? Does not $B$ in turn require justification, or if we take the two propositions $\mathrm{A}$ and $\mathrm{B}$ as now forming a connected whole, does not this whole stand equally in need of something further to substantiate it? If so, we shall need a third proposition $\mathrm{C}$, and we shall be no better off, since as soon as $C$ is asserted the same question will revive. Thus we are threatened with an endless series in which, though always proving, we never get any nearer to the grounds of proof. From this there are two possible ways of escape. One is frankly to admit exceptions to the general require- 
ment of proof - to recognise the existence of self-evident first principles, to trace other thoughts, judgments or affirmations if we can back to these, but to treat the principles themselves as self-evident. But this method has its weaknesses. To begin with, self-evidence is a term of doubtful import. Taken quite literally, it suggests that the evidence is in the truth itself, and that it is an objective quality, say, of a relation between two terms which the truth expresses. If any truths were so stamped or hallmarked with inherent certainty and primacy, they would, indeed, occupy a peculiar position. But the bare conception of 'evidence' implies a mind which is convinced. Even if the hall-mark were there, it would not be a mark of self-evidence unless there were a mind to which it appealed. If so, two factors at least go to the composition of self-evidence. It is not the simple and unanalysable thing that it appears, but depends $(a)$ on the character of the relation asserted, $(b)$ on the mental make-up of the thinker who forms or accepts the assertion. Now the mental make-up may be affected by much that is external and accidental. In the judgment of value in particular it is coloured by emotional elements, prejudices, interests, sympathies and antipathies that together form a very composite whole. This whole may react upon a very simple proposition with an affirmation or rejection of luminous intensity, endowing the response with a strong feeling of subjective certitude. But this felt certitude-felt by the mind in making the affirmation as due wholly to the intrinsic character of that which is affirmed-will, in fact, be attributable to an intricate maze of psychological forces, and to assume that those forces necessarily guide the mind to truth is to take a great deal for granted.

Owing to the psychological complexity underlying felt certitude people do, in fact, differ largely in their opinions as to what is self-evident. To some, for example, the existence of God and the immortality of the soul appear as certain as their own existence. To others, both affirmations appear highly disputable. About virtue, duty and the good conflicting propositions have been taken by different people as accurate. These divergencies are often 
explained as arising from confusion and mutual misunderstandings. In the case of the good, for example, it is suggested that people are really agreed about ultimate ends, but differ as to means, while misunderstanding on the ultimate question arises from the confusion of means and ends. But the admission of such confusion is fatal to the inherent sufficiency of self-evidence. It may be that there is always a kernel of truth within the husk, but if so, we must be sure that we have stripped off all the husk before we proclaim our certainty. That is to say, our axiom must be subject to criticism, and criticism means comparison and interconnection with other judgments, other data of experience or products of thought. We can no longer take the self-evident as an isolated datum. We have to treat it as part of a comprehensive system of thought wherein it may undergo correction.

The difficulty that appears in this view is that we seem to have no fixed starting-point or given basis for the operations of thought. Instead of being furnished with first principles, which we can apply without any shade of doubt, we have to build up our principles as we go along, and it is hard to see how, in so doing, we can escape a vicious circle. If, however, we analyse the conception of rationality more closely, we shall see that on the one hand it excludes the notion of an axiom detached from those forms of connection with the totality of experience which constitute proof and explanation, while on the other it enables us to understand how our thought-system takes gradual shape by the mutual determination of its parts rather than by crystallisation around a core of unchanging principle. To understand this result, let us conceive the rational principle at work on a limited scale. Let us suppose that we form a judgment, no matter what or how, so that it be a genuine thought, asserting, let us say, some relation between two terms, and held with a certain degree of assurance or conviction. Now, if we are asked for a reason or ground for this judgment, we naturally look to some further thought or some further experience that can be rendered in thought. We do that alike whether we wish to prove the original judgment or to explain the relation which it asserts. Proof 
and explanation, the two aspects of the work of reason in thought, appear to take us outside the content of what is to be proved or explained. But if this process be generalised, it inevitably leads us to something which is neither proved nor explained. In proof of proposition A I adduce proposition B, and in proof of B I adduce C, and so forth as long as may be. But wherever I may have arrived in this process, my first proposition is always unproved. Similarly I would explain an event, and I do so by referring it to B, and $B$ I refer to $C$ and so on. But wherever I may have arrived in the process, the event from which I start is always unexplained. This way of conceiving reason, then, leaves its work necessarily incomplete; there must be something unreasoned. It also leaves it dependent, for what is reasoned out follows from what is not reasoned out.

This leads us to ask whether there is not another way of regarding the work of reason which is not thus selfmutilated. Let us, as before, suppose that we have formed a certain judgment $A$, which we hold with a certain measure of conviction. We ask, however, for proof of it ; we find another judgment B which is formed independently, again with a certain measure of conviction, and which on comparison is found to necessitate $A$ as a consequence. Now if we take $B$ as certain, as a definitely established truth, $A$ is established along with it, and the question of truth or falsity is closed. But even if B is not certain, if it is only probable, still its probability will affect our belief in. A. Provided that that belief has anything short of the maximum of intensity, it will be strengthened by the corroboration of an independently probable argument, just as it would be pro tanto shaken by conflict with some independently probable argument. The degree of validity which we reasonably attribute to $A$ is, in short, dependent on two factors, ${ }^{1}$ on the force of conviction with which the

II assume for the sake of simplicity that the judgment A is immediate in the sense that it rests on processes which cannot be analysed out. If we suppose this analysis to be effected and all the empirical data and psychological laws contributing to the judgment $A$ to be set out in the form of explicit judgments, then the validity of A would simply depend on all the independent judgments leading up to or corroborating it, each one of these having a definite felt force of its own. 
judgment is impressed on us by whatever experience or processes of thought lead to its formation, and on the force of other judgments which corroborate or conflict with it.

So far we have supposed merely that the first judgment, which we have called A, is necessitated by the second $B$, and this leaves us with the alternatives of either seeking a further ground for B or assuming its truth without proof. But there is a further possibility. It may be that while $B$ necessitates $A$, it is equally true that $A$ necessitates $B$. If that is the case, we have a consilience of two independent judgments, and the result is a miniature system in which the several parts imply one another. In this system there is no part without some rational justification. For if we start with $\mathrm{A}$ we find it corroborated by $\mathrm{B}$, and if with $\mathrm{B}$ we find it corroborated by $A$. Of course, if we only believe $A$ because we believe $\mathrm{B}$, and believe $\mathrm{B}$ only because of $\mathrm{A}$, this would be to argue in a circle. But if we believe each independently on its own merits, and if they corroborate one another, the case is altered. Starting from different sides they meet in one point. The fact of their consilience tends to substantiate both at once. Their respective contents throw light on one another. We are no longer proceeding in a linear series, proving one proposition by means of another which is unproven and unexplained. We are moving within a miniature system, each part of which necessitates the other, and no part figures as an absolute 'beginning,' nor does any necessarily point for explanation to something outside the system. If this is so, the system $A B$ is a rational system devoid of that selfmutilation which we found in the deductive 'series.'

As long as our conclusions depend wholly on premisses, and these on further premisses, until we come back to first principles, our reasoning forms a chain which hangs from a fixed support. But the support itself is non-rational. No reasoned account of it is or can be given, and no completely rational system can therefore be formed on this method. It is only when each element in a system necessitates and is necessitated by the remainder that the non-rational element disappears. Every judgment affirming some element in such a system has a rational ground, and the same may be 
said of the thought which conceives or the judgment which affirms the truth of the system as a whole. It is a reasoned judgment-reasoned not because it depends on some outside truth, but because it is inferable from any of its parts. Thus, in our miniature system of two judgments, if we assert $A$ it gives us $B$, if we assert $B$ it gives us $A$. In either case the second judgment substantiates the first, and in both we have the whole $\mathrm{AB}$, the result of two independent corroborative judgments. A rational system of thought appears to be generically a whole of this kind.

Such a system can only be negated or modified by a judgment drawn from an independent source. Hence, if it included all experience, it would be finally established. If it included all human experience it would be established as fully as human experience at any given time could establish it. In point of fact, any system at which we can arrive is never so complete as this. Our contact with the real world is partial and sporadic. From a heterogeneous experience we get a multitude of glimpses and partial views, and it is but gradually and slowly that we bind them together. It is, however, this work of binding them together that constitutes the distinctively rational in the human mind. It is irrational to divide up thought in such a way as to take any part in complete isolation from the remainder. It is irrational to take any partial view as final truth without considering the bearings of other views derived from other sources. We may even say that it is irrational to be contented with the results of our partial experience, however perfect its internal coherence, instead of actively seeking fresh data from fresh experience. Conversely, it is the positive work of reason to be for ever organising our experiences into a systematic whole of thought. This is as much as to admit that the work of reason is never done, that it is permanently operative in the way of bringing all manner of experiences into relation with one another, but that the total view of the world which it forms-or even that which it could form if its synthesis were far more nearly perfect than it is-is not, and, for a limited mind cannot be, final. What is definitely established is not the totality of thought achieved at any given 
time, but the principle of organising experience as a whole. In proportion as this principle is carried further we reach, not the truth, but a step on the way to truth - fuller knowledge, deeper insight, more articulate expression. It is in this sense that thought, as an interconnected system, is valid.

Nothing has been said so far of the methods by which judgments are formed, and interconnected. But it was pointed out above that an act of interconnection, of inference, for example, is the expression of a felt mental necessity which may be regarded logically as the equivalent of a judgment stating that the conclusion of the inference follows from the premiss. Inferences are very often faulty, but when by analysis and comparison those which are mutually inconsistent are separated out and those which coincide and so necessitate each other are formed into a general statement or law of thought, we have in such an axiom the expression of the consilience of a body of judgments (or their equivalents) habitually formed by the human mind. These laws have further to be compared with one another, and it has to be seen whether contradictory results arise in applying them to experience. These are tests positive and negative of consilience and mutual consistency parallel to those applied to the judgments which it is the business of the methods to connect. We cannot prove the validity of logical methods by deducing them from something else; we can substantiate them by showing that they are consilient.

The principles which embody these methods will be the legitimate principles of reasoning, and the body of thought formed on these principles will be rationally formed and is rightly held valid. But this is precisely the conception of method to which our analysis of the actual logic of science led up, and into which it has now been our business to examine.

The view of rational thought put forward here is confirmed by the actual character of our knowledge, and its points of strength and weakness. In the rough our common-sense knowledge forms a coherent system; that is to say through 99 hundredths of our daily life we find our grounded expectations fulfilled. Our world is orderly, 
and the senses of sight, touch and hearing supply us with information about outer objects which in the mass corroborate one another. The coherence, however, is not complete. The abnormal plays its part, and there are departments of the environment, like the weather, where mutability reigns. The lack of completeness leaves an element of uncertainty in the domain of common sense, and forces the candid to acquiesce in the judgment that, after all, probability is the rule of life. The endeavour towards a more complete, and also a more express and conscious coherence, takes us into the region of science and of philosophy. Here the true character of coherence tends to be masked by the impulse to find a single first principle from which a department of truth or perhaps (in philosophy) the whole of truth may be deductively inferred. This impulse is in reality due to a one-sided apprehension of the idea of systematic unity. What appear as 'first' principles are, in fact, based on the harmony of experience which they themselves reveal. They are neither a priori truths nor mere assumptions which turn out to be consistent with experience. They express the pervading unity in a system of judgments shown, in the manner indicated above, to necessitate one another, and such a system we now see is precisely what we mean by a rational and valid body of thought.

We have now seen in what sense it is possible to meet the demand that a reason shall be given for all that we think. It remains to consider why and in what sense it is irrational to let our thoughts be determined by our desires, emotions, or, in fact, by anything proceeding from our own peculiar mental make-up rather than the intrinsic character and relations of the objects asserted. The most obvious objection to this element in our definition of the irrational is that reason itself - our connected system of judgmentswill force us to recognise facts which depend for their bare existence on 'our own peculiar mental make-up.' Any fact of my own consciousness, any feeling or emotion, for instance, comes into being because I am so constituted as to feel it. There may be an 'external' exciting cause, but the feeling is the reaction of the conscious being upon it, and 
there are countless individual differences in such reactions. What causes pain to one may be a source of pleasure to another, but the pain and the pleasure are alike, for the time being, facts. If I make a mistake or suffer from a hallucination, the mistake or the hallucination are none the less facts within my consciousness. The reply is that they are not irrational as facts but only as judgments. What is essential to truth is that they should be recognised for what they are, that is to say, that the assertion made-' I feel pain,' 'I see a ghost,' should be recognised as states of the person making the assertion, and dependent on his mental constitution. So recognised, there is nothing false about them. Error comes in when the assertion takes something which depends for its existence on the nature of its own mind for something independent of that constitution. If the error is eliminated by allowance for the contributory cause the assertion becomes true.

A second and more subtle objection is that knowledge of the truth itself depends on our ' mental make-up.' Knowledge is a state of mind, and is arrived at by mental processes, and may even be said to be attained under the influence of feeling or desire-viz. by the impulse to investigate and the interest in truth. There is, in this sense, a 'subjective factor' in rational thought which cannot be eliminated without eliminating thought itself. These processes and impulses, however, are ex hypothesi not those 'peculiarities of the mental make-up' which disturb our judgment and cause its assertions to diverge from the real character and relations of the objects asserted, and it is these peculiarities, and these only, which have to be eliminated from the work of rational thought. What is irrational is to maintain any assertion without regard to any peculiarity in the constitution or attitude of the asserting consciousness which might cause divergence from the truth. The implication is that truth is objective, i.e. something independent of any opinion that might be formed about it. Except for the facts of the individual consciousness and the changes which the individual has set up in the outer order, the system of truth would remain unaffected by the removal of the individual from the world. Whatever, then, is at 
work in the mind of the individual to cause divergence of opinion from this standard is included in the conception of the subjective factor in judgment, and it is in this sense that a rational order requires the elimination of the subjective factor.

We may, in fact, take the conception of objective Reality as the central point towards which all our distinctions of Rational and Irrational lead us. The principle underlying all reasoning, that is to say, is that Reality is a single system of interconnected parts, which it is the function of thought to apprehend. Hence all known facts must ultimately stand in connection with one another ; none must conflict with another; no way of interpreting reality that is true can ultimately conflict with another that is true ; and divergence of view; arising from the constitution of the mind itself, involves error to be removed by eliminating this subjective factor. This being the nature of Reality, it follows that its rational interpretation will take the form of a connected system of judgments. It does not, unfortunately for human knowledge, follow that any connected system of judgments must contain final truth. What does follow is that we know the goal of rational endeavour and the norm which distinguishes rational from irrational processes.

The ideal of the reason then is a system of consilient thought, interconnected by methods which themselves, as shown above, form a consilient system. But actual thought falls short of this ideal. We constantly find that the harmony arrived at from certain data is disturbed by contradictory results, and that some readjustment becomes necessary, in the process of which we often discover that our original system was insecurely founded. Thus construction constantly involves criticism, correction and reconstruction. The general principle of such reconstruction is simple enough. It is simply that of the impartial application of the idea of consilience. That reconstruction which will overcome contradiction and reintroduce not merely consistency but consilience is rational. But the difficulty that arises is this. If a body of thought which is internally harmonious may yet in contact with fresh data 
prove to contain error, at what point can we be sure of attaining final truth? Even if the whole of our present experience had been reduced to order, which is far from being the fact, might it not be exposed to the chance of subsequent correction? And if this be admitted, where is our ground of confidence? The answer is that the validity of thought is not that of finality or achievement but of growth. The most general expression of the rational impulse, which sums up all reasoning processes and depends for its validity on the fact that it does so, is the impulse to establish intellectual harmony. This impulse is not defeated by error, because under its control error is always partial truth, leading by its very imperfection to further investigation and correction. An error may, in fact, involve more insight and a larger grasp of experience than a truth that is maintained without insight into reasons, and in the pursuit of the consequences and implications of error we get back to a wider and deeper truth. Thus the ultimate basis of our thought is not one of certainty in assignable net results, but the conviction of the justification of the impulse towards harmony, which conviction is not contradicted but corroborated by the actual course of intellectual history. The organisation of our experience in this view would remain a valid and a rational process even if none of its results were final in the form which they assume at this moment. Rational thought is no longer limited to the apprehension of a fully and finally established system. It becomes rather an impulse working towards an ideal, organising the acquired results of experience into a coherent whole, and extending them by persistent investigation.

Thus Reason in general may be briefly defined as the impulse towards interconnection.

Thus the idea of development lies at the very basis of validity itself. When critically examined the certainty which one ratiocination claims is found to hold good only with this saving clause, that it is understood to yield truth not final and complete but partial and in growth. By consistently using our reason we attain not necessarily the truth, but a truer view. The wider the basis and the more complete the articulation of thought, the more just is its 
rendering of reality - that is the final implication of the rational process. This, of course, is not to deny finality to all truth whatever. There are, as shown above, truths as to which no experience, direct or indirect, specific or general, suggests a doubt. We may justly believe such truths to be final, but we must distinguish belief in finality from finality in belief. We may justly disbelieve that any reconstruction will affect the meaning or value of certain parts of our thought, but this disbelief does not possess final certainty. What has final certainty is the belief that the development of rational thought yields advance in the partial knowledge of reality, and not till this advance has reached some higher point of view can more be said.

8. There is, however, an ambiguity in the use of the term 'rational impulse' which remains to be examined. We speak of 'establishing,' of ' seeking' or of 'coming to appreciate' interconnection. These terms are not really convertible. It is true that commonly we speak of 'establishing' a law, i.e. of discovering and proving some general relation to be true. In this we speak as though we were actively creating something. Yet the very point that we establish is that the law holds, and always will hold, whether we believe it or not. We are not then establishing or creating the law. The only thing we are creating is a thought in ourselves and in others which recognises that law. It is only on this side and in this limited sense that the reason is creative in the sphere of knowledge. In the sphere of action it has a wider scope. For the rational impulse has a practical as well as a theoretic application. On the practical side its object is not merely to interpret or appreciate existing interconnection, but to alter, transpose, abolish, create or modify so as to form a new kind of system, a new order in Nature or human life. To give a generic name to the element which prompts and controls action we may call it feeling, and say, again to use the term in a very wide and generic sense, that feeling prompts to such action as serves its satisfaction or removes causes of dissatisfaction. In the permanent satisfaction of feeling there is a relation, which we may call harmony, between 
the feeling and its conditions, and we mean here by harmony a definite mutual support between a succession of feelings on the one hand and a set of conditions out of which the feeling arises on the other. The feeling is at the root of efforts to create or maintain these conditions, and the conditions as they are realised give rise to the feeling. We may thus consider satisfied feeling as a state of harmony between the mind and certain conditions (whether external or internal) that affect it, and dissatisfied feeling as a disharmony. Now if we seek for a moment to imagine that there were only one mind in existence, and that it could experience only one type of feeling secured only by the presence of certain conditions, the whole work of reason on the practical side would be that of supplying the knowledge which would be utilised as a means to securing the requisite conditions. So far there would be no particular object in introducing the conception of a practical reason or a rational impulse in practice. When, however, we consider, even within the limits of one mind, the possibility of many types of feeling, which may rest on discrepant and even contradictory conditions, a new question arises, which feeling is to be preferred, and why? We need now a rational ground of preference among satisfactions or feelings, and if we are to apply our former principles we shall look for a connected or systematic order, which satisfies as a whole, in which subordinate or constituent elements of satisfaction find their place in relation to the whole, and in which no discord or contradiction of feeling with feeling is tolerated that cannot ultimately be resolved into a more deep-lying concord. The only difference will be that here the principle of interconnection, the test by which consistency and inconsistency are to be judged, is that of practical reconcilability. Feeling must harmonise with feeling, as each feeling harmonises with its conditions. There must be the relation of practical mutual support throughout the order. The impulse of the Practical Reason will then be to establish a practical harmony, a life of feeling in which the parts are so interrelated as to form a connected whole. Lastly, if we introduce the conception of a multiplicity of persons or relatively indepen- 
dent centres of consciousness, no difference of principle emerges. There is only the further possibility of conflict or harmony as between the feelings of different persons, just as before there was the same possibility as between different feelings of the same person. The rational impulse in its practical application will remain the same. It will be the impulse to constitute an order dominating the world of mind as a whole in all the centres of consciousness in which it lives, an order which as a whole satisfies the mind, in which all constituent elements of satisfaction find their place by their relations to one another and to the whole, in which no discordant element is allowed a place. The practical impulse is impulse guided by feeling, and if there is a rational impulse in practice its work must lie in the direction of establishing a harmony in the medium in which it works, that is to say, in feeling wherever found, and that, again, is as much as to say throughout the sentient creation. The impulse of reason then is towards the establishment of a harmony throughout the world of mind, and this harmony rests on two conditions, (I) on the harmony of feeling as between one mind and another, and as between any one mind at any moment and itself at any other moment, (2) on the harmony between natural conditions and the requirements of feeling whether those natural conditions belong to the physical environment or to the structure and functions of any given mind itself.

To sum up. In cognition the rational impulse is to appreciate a connected system. In practice the rational impulse is to establish a harmonious system. What is rational is the interconnection of elements in a pervading unity. In cognition we have the impulse to discover this interconnection as a permanent reality. In practice we have the impulse to create it in the shape of the unity of that Feeling on which generically all impulse rests. The point of difference being understood, we may speak of the general function of Reason as that of Correlation, or of bringing elements together into a connected whole.

The ethical order then is rational just in the same sense as the cognitive order. That is to say, both have an ideal towards which they work, and that ideal is one of the har- 
monious interconnection of elements. So far as such interconnection is achieved both can claim to possess objective truth, i.e. truth independent of and superior to opinion or (on the practical side) desire, though neither can claim finality in their rendering of the truth. On the practical as on the theoretical side then we take the movement of mind to be a movement towards truth through progressive harmony. But on the one side the aim of rational construction is an appreciation, partial but within its limits just, of the Real Order, on the practical side it is the appreciation, as an object of effort, of an ideal which is rationally justified, and founded on the real conditions of the spiritual order. On this side also the appreciation is partial, and what is held good is so held subject to the fuller understanding of the conditions of development. In both cases the movement of mind may be regarded as a movement towards reality, in which the appreciation of its own development is the final condition of a just orientation. It appears consequently that the movement as a whole is founded on conditions in the nature of reality, and has therefore the elements of durability and persistence. But this inference opens deeper questions, to the examination of which we now turn. 


\section{CHAPTER III}

THE PAST AND THE FUTURE

I. WE have traced the development of mind from the first efforts of adjustment to sense-stimuli in the individual to a point at which the entire collective life has become in conception a unity. At this stage a body of thought has grown up which is in principle a valid though partial interpretation of reality, and a system of conduct which is similarly a partial interpretation of real values. So far the movement of the mind is seen to be founded on the real order, and this is at least one condition of permanent advance in the same direction. What are the further conditions? What are the capabilities of development in the social mind, and what ground have we for the belief that these capabilities will be fulfilled? The first reply that suggests itself runs on purely empirical lines. We have traced the path of orthogenic evolution a long way. We have seen it describe a certain orbit, and we may infer that this orbit will be prolonged. We may expect then that the stage of self-conscious development will complete itself, and prepare the way for a still higher and wider spiritual synthesis as previous stages have done. Mind as an organising principle will continue to grow indefinitely. But so stated the inference is hasty and precarious. A curve cannot be produced with any certainty unless its law is known, and we have not as yet been able to trace such a law for the advance of mind. We have described one condition of this advance, viz. that rational thought is founded on real relations. But for the rest what we have done is to determine the direction and magnitude of the 
movement, but not its causes. We cannot even say that it is continuous, for we see its movement broken by many hesitations and backslidings of too great importance to be overlooked or dismissed as casual irregularities. Indeed, our whole conception of evolution as a process in which mind is only one, though a growing, factor militates against the acceptance of an automatic tendency to steady progress. On the other hand, it would be absurd to dismiss the evidence of past development in forecasting the future. If development of a certain kind has occurred, it is certain that the conditions which render it possible exist, and if the development in question has proceeded on a very great scale through long periods of time and over wide diversities of environment, it is a necessary inference that, whatever its conditions are, they are of great permanence and high generality. Now, keeping closely to the empirical results and without any hypothesis as to the nature of the permanent evolutionary forces, what we are able to say as the result of our descriptive account of mental evolution is this-that tracing the growth of mind from the germ upwards, we find an extension, not indeed continuous, but proceeding by successive stages of vast moment, of the sphere of conscious control of racial life. This growth and, therefore, the conditions rendering it possible, run through the entire history of mind and its environment as we know them from first to last. Thus as an empirical generalisation we are justified in the hypothesis that these conditions are permanent, or at least of very wide reach.

But there is no need to leave the problem at this stage. In point of fact, our descriptive account of the process of development does yield a theory of the conditions, though these have not yet been explicitly set out. Thus, to begin with, we have found that, point by point, the control of mind is limited by its scope. The individual organises his life with a certain measure of freedom in so far as he is able to utilise past experience and to bring within his mental grasp that in his future which vitally concerns him. He fails in so far as his grasp is too narrow or as his purposes are not accurately adjusted to his real needs. Now in our highest stage we assume a mind of scope so wide that these 
sources of failure are blocked up. We assume that it has as a basis to work upon a complete understanding of the conditions of its own development, and that its purpose is a harmony of the elements of value discoverable in the millions of lives that make up its unity. We assume, that is, a scope equated with possible experience, and may we not, along with such scope, assume the corresponding power of control? May we not then infer that growth will continue, because now we have, what we had not before, a sufficient force to secure it?

For the purposes of this argument, however, two conditions are necessary which have been tacitly postulated in this statement, but which are by no means to be assumed without criticism. First, it is assumed that the stage described is complete, that there already exists that fullness of knowledge and rational completeness of purpose which we require to assure us of continuance. It need hardly be said that the reality is far different. This stage is only at its beginning. The organic union of humanity is still an ideal embodied in mere filaments of actuality. The understanding of developmental conditions is equally in its infancy. How can we be sure that either of them will grow to maturity? If we assume that they will grow further because they have grown so far, we are back in the line of argument discarded above. If we say that they themselves contain the promise and assurance of growth, we apply to the germ what could only be true of the developed state. And there is a further point, which will bring us to the second tacit postulate. Our knowledge of developmental conditions is admittedly incomplete. So far we have seen no absolute barrier to further expansion. But it may be that this is only the result of our ignorance. Suppose that there are, for example, physical conditions which set an absolute limit to the growth, perhaps even to the existence, of mind. What could the advance of knowledge do with these conditions except enable us to recognise them with a more fatal clearness? Suppose, for example, that the energy available for human needs is a limited and diminishing quantity, suppose that the conditions of life upon the earth are transitory, and there exist no means of 
permanently arresting vital decay. By this I mean not merely what is obvious, that any such means are far outside our present ken, but that the advance of knowledge brings us to a point at which we can demonstrate their eternal impracticability, while at the same time foreseeing clearly as the alternative the final extinction of the human species. Suppose that this impasse is the result to which our completed knowledge brings us, and it becomes evident that in place of an indefinite expansion of mind we must conceive a barrier, remote, perhaps, but rigid, arresting the line of advance on which we have hitherto moved. Conversely, to prove that progress may go forward without limit, we must know that there are no such barriers, but that the conditions of existence are indefinitely malleable by adequate knowledge, a thing which we can by no means assume.

The case then stands as follows. The narrative of evolution leads us to conceive the maturation of a Social mind in complete control of the conditions of its own development. Given (I) that such a mind were actually evolved, and (2) that the conditions were malleable without restriction, it would be for its own purpose all-powerful, and would, therefore, with certainty achieve progressively the perfection of life. But (I) the evolution of such a mind, though it has made a certain advance, is very far from complete, and (2) we do not know, and have not, indeed, yet enquired, how far the conditions are malleable and how far repugnant or conducive to the further development of Mind. Both questions refer us back to the general conditions of Development.

2. Now the ideal has been defined as a Harmony in the entire life of mind, and the question is whether the conditions of evolution make for or against such a harmony, or whether, finally, they are such as to render harmony possible under the control of intelligence, though not otherwise. Harmony is defined as mutual support between two or more elements of a whole. If these elements are unchanging, their mutual support tends to maintain them unchanged. If any of them consist of internal conditions, 
which in their interaction produce an orderly series of changes along a definite line, the support of the other elements is something that furthers that development. This principle is co-extensive, not merely with the activity of Mind, but with the organic world. The organism, as will be shown more fully later, is a harmony which is perfect or imperfect according as the organic unity is complete or incomplete. But throughout the organic world harmony is shot through with discord. The cunningly arranged harmony of the parts and processes of the individual living being only enable it to prey more successfully on other living beings. ${ }^{1}$ But, as we have seen, the advance of Mind is measured by the constant extension of the sphere of harmony and the removal of partial disharmony and discord within that sphere. There is here a double advance, the general conditions of which are very simple. (I) As far as two things support each other, they have an advantage in the struggle with others which conflict with one another, and their type will tend to multiply. The advantage, moreover, increases as the harmony widens, and from being very small may become the decisive factor. (2) What applies to concrete individuals applies also to principles, tendencies,

${ }^{1}$ In the lower stages this rivalry appears as contributory to the development of the successful types. Hence the view that natural selection is the cause of progress. If this were true progress must be a self-defeating process, because the struggle for existence on which natural selection depends is the negation of harmony. The truth is, as argued further on in the text, that harmony always involves some selection, but (a) not a selection determined by the law of force, $(b)$ not necessarily a selection involving the destruction of any other members of the species, but only modification of their character.

I have put it that rivalry 'appears' contributory to progress in the lowest stages. Is it only appearance? I confess to thinking a more radical view preferable. According to this view progress at any stage depends $(a)$ on variations due at bottom to the efforts of the living being in the lower stages to maintain, in the higher to extend and perfect itself, (b) on the suitability of the resultant variation to conditions. It is this relation of variation to conditions which we have constantly used as the explanation of reflex, instinct, sentiment, custom and so forth. Thus it is not the extinction of other types but the suitability of the higher type at each point which is the condition of its advance. At most the elimination of the lower would only be an indispensable condition as long as the food supply is insufficient for both. 
modes of action. So far as these conflict, they tend to cancel out. So far as they harmonise, they maintain one another. Hence within any system working on the whole in co-operation, the harmonious tendencies survive and the harmony becomes more and more complete.

Thus harmony is not only a product of development, but a cause of development. It is a cause, so to say, of itself, for it tends, in the manner shown, to extend its sphere and deepen its hold. But harmony does not grow by any automatic process. The living being and, indeed, the structural parts of the living being tend, in the first place, to maintain themselves, and it is only by selection and modification that they are brought into harmony with one another. The possibility of harmony thus depends on the plasticity of organic types, and in the lower stages, where this plasticity is small, it cannot advance far. In the higher stages, and particularly among men, the potentialities of development become more numerous and many-sided, and it is possible to select among them those that will harmonise, and so progressively extend the principle. The development of harmony then involves a principle of selection or modification. In the lower stages such a principle is found in the indirect action of heredity, which preserves the variations suited to their environment, and, therefore, among others those which depend for success upon an extension of harmony. But the wider extension of the principle rests on consciousness, which, as the direct organ of correlation, is the means of harmonising the diverse promptings of different structures and the independent aims of different living beings. But even when consciousness has arisen, the law of self-maintenance remains. Every type of life, even every type of action and of structure, tends to maintain itself, and so every fresh advance of harmony which is to replace discord involves modification. It is of the nature of a discovery of a new possibility of synthesis for which the conditions may be long preparing. Hence a system - whether physical or social - which is strong enough to maintain itself at a certain stage may remain there indefinitely till new conditions arise. More- 
over, if the internal harmony is imperfect, it may at any period begin to decay, while it is always subject to disruption by external assault.

Thus harmony, though it gathers strength as it goes on, does not assure continuous progress. On the other hand, in the world of mind every felt disharmony is a stimulus to effort. Instead of merely threatening destruction, it is at least potentially a cause of advance. Yet the social mind does not advance steadily. In general terms the reason for this failure appears to be double. On the one hand the method of dealing with the trouble may be unknown and so remote from existing ways of thought that it fails even to prompt research. Thus people may live for ages in a volcanic region without beginning upon a seismology. On the other hand, the partial order that has been created may itself inhibit further advance. Thus a general survey of savage life suggests that the main responsibility for the arrest which has retarded so many races, is to be shared between the belief in witchcraft and the practice of blood-revenge, which between them keep early society in constant tension and disorder. Yet the belief in witchcraft is a necessary result of normal thought-processes at a certain stage, and blood vengeance is the first known method of securing any rights at all. It is needless to remark that the gods and kings who superseded the witches and avengers of blood are in turn potential obstacles to further advance.

3. Progress then is an evolution of harmony. This is a self-furthering process in the sense explained, but is none the less subject to arrest by causes of discord within or without. In all but the lowest stages it is effected by conscious correlation, and its development depends on the extension of the sphere of conscious control. As to the conditions and consequences of this extension our review of development has given certain results which may be briefly summarised.

i. Consciousness arises under the conditions of physical life, and in the first place as a means to secure ends subordinate to the general struggle for existence. But so far 
as the sphere of consciousness extends, it establishes a harmony in which feeling is the pivotal point.

ii. The conditions (whether in the constitution of the individual or in the environment) under which consciousness at any stage subsists prescribe the general direction of its activity, except in so far as these conditions have themselves come within the grasp of consciousness. As between any distinct centres of consciousness (whether in different individuals or in the same individual at different times and in different relations) there is no necessary organic connection, and the aims of conscious activity are correspondingly discordant.

iii. The development of consciousness in its principal phases has as its basis an enlargement and a redirection of activity depending on the absorption into the body of consciousness of some of the conditions which have previously operated upon consciousness from without. The effect of this change is in each case an extension of harmony.

iv. Conditions which, under the selective action of consciousness, become conducive to harmony limit its action and thwart its development as long as they remain outside its grasp. Among them the most important is the existence of distinct centres of consciousness, which, until they are brought into relation, have discordant aims and cancel each other's efforts.

$v$. In the highest stage the redirection which occurs lies in the systematic effort to absorb the entire conditions of development itself. If this were successful there would be no 'external' conditions left to operate. The sources of disorganisation would be removed, and orderly progress would be assured by the complete harmony of interacting parts.

vi. Thus at any stage there exist conditions of further growth which need a further condition to complete them, viz. that they should be understood. If it be admitted that Mind has arrived at the point at. which the conception of development becomes the basis of its operation, we have the pre-existing (hitherto external) conditions completed by the new condition that they are recognised, and we are, 
therefore, in possession of the principle necessary to complete the intelligent control of life, and it needs only to work out its application. According to our previous argument, which showed that each advance in harmony makes the next step easier, this application, far from foundering on any impossibility, should become more rapid and certain as it proceeds.

Our argument, it will be seen, does not show that the movement towards harmony proceeds like a physical action independently of human choice. It shows that it proceeds through human choice. Formally stated, (I) our analysis of the facts shows that it is possible, (2) our analysis of values shows that it is good, that is, holds it up as a possibility at which mankind should aim, (3) our analysis of the motives that determine that social mind goes to show that what is clearly propounded as good will in the end be adopted, and only in this sense and on this condition can we predict. We may conclude that the ideal of harmony tends to realise itself, and that with progressively diminishing difficulty, through the extension of intelligent control.

4. But behind this result arises a larger and more difficult question. We have shown that harmony, so far as realised, is a factor in success. We have shown that the possibilities of harmony can be extended by intelligence. But we have not shown how far they can be extended. We have shown that the conditions are malleable, but not how far they are malleable. We may assume that the mind can ultimately so far control its own action and its own products, such as social institutions, as to achieve a complete internal harmony. But we cannot thus assume that it can also control the physical conditions of life. May it not be that the upshot of the most complete understanding of reality would only be to show that there are elements which refuse to be harmonised with the aims of mind, that there are physical or biological limitations which set a term to development and even to the existence of mind itself? Say that our argument so far has gone to show that the social mind may, and probably will, attain a condition of complete internal harmony, together with such control 
of the conditions of its life as the utmost extension of knowledge renders possible. What are the limits of this control? May they not be seriously cramping? May there not be biological laws recalcitrant against control, which introduce an insuperable obstacle even to the work of social harmonisation and ultimately engender an arrest and decay on the large scale, as history shows us instances of arrest and decay on the partial scale? Beyond these, are there not physical conditions, the dissipation of energy, the cooling off of the earth, which we can never control, and which stand as an alte terminus haerens to all progressive movement, and even to the span of conscious life? Of the positive evidence of such conditions I shall say little. I note that within my own lifetime some of the barriers supposed to be most adamantine have crumbled before the advance of knowledge. Thus, as to biological conditions, down to my own time the argument derived from Malthus was supposed to present an insuperable difficulty. Whatever the temporary advance of comfort, it would be swamped for the masses by the increase of population, and every social reform resting on a deepened sense of unity and a more generous impulse of mutual aid would only defeat itself the more rapidly by the impetus that it would give to the multiplication of devouring mouths. This line of argument, which for three generations served as an intellectual stronghold of obstruction, has crumbled before the actual fall of the birth-rate, as a result of those very improvements which were to flood the world with hungry children. The boot is now on the other foot, and the pessimists have to harp on the possibility of race suicide. As to the pessimism of physical science, recent discovery has taught another valuable lesson. The speculations of Lord Kelvin, deriving an appearance of demonstrative cogency from their mathematical form, led men to conceive the earth as relatively short lived, and the present age as a late stage of its existence. In vain men like Huxley pointed out that the entire cogency of Lord Kelvin's reasoning was in the method of deducing conclusions from its assumptions, that these assumptions were unverified, that they were valid only if our knowledge of 
the sources of the earth's heat were complete, and that there was no ground for assuming any such completeness. The dogmatism of the mathematician prevailed until the discovery of radio-activity, revealing entirely new sources of heat, proved the justice of Huxley's caution, and placed the whole question of the terrestrial past and future in a new light. We have now every reason to think that the durability of the earth as a habitable planet is immensely greater than Lord Kelvin supposed, that it is to be measured in hundreds rather than in units of millions, and that we are in no sense witnessing the latter stages of evolution on a dying planet. It may be said that, nevertheless, ultimate decay is certain, but it may be replied that the supposed certainty once more arises from drawing mathematical deductions from facts supposed to be known in their completeness, and the lesson of radio-activity is precisely that we may be very far from so knowing them. As to the Dissipation of Energy, this is still more clearly an incomplete account of the world-process as a whole. For it can proceed only by assuming an infinite quantum of original energy at high potential, of which it pretends to give no account whatever. Its validity is merely in the account that it gives of mechanical process as such, and the more certain it is the more it proves that mechanical processes cannot exhaust reality. It proves that there must within the sphere of reality be, or at least have been, an unknown compensatory process building up what mechanism dissipates.

Neither can we, in face of modern inventions and of our whole account of the growth of mind, set any limit to the possibility of the control of external nature. It may seem grotesque to suggest that the time may come when man will control the movements of the earth or at need accomplish migration to another planet. But a few generations ago it would have seemed equally grotesque to fancy a means of communication across the ocean without so much as a visible connecting mechanism. What can fairly be said against an optimistic view of the future of human control is that it is not impossible, but unverified. What reason have we for adopting it? Why should we think 
that the constitution of things is such that in time Mind is to have the ultimate sway? Have we any such reason? Can we connect the development which we have followed with the world-process as a whole? Can we base it on conditions that are not merely of wide reach but eternal? To ask this question is to attempt nothing less than to discover in essentials the nature of the moving forces which have determined the whole vast sequence revealed to us by human history, by the study of the animal world and by the geological record, which has made up the life of the world in time. Even had we no question of the future to raise, the actual emergence of so much of life and intelligence as we know, the gradual peopling of the earth with beings of a higher and higher consciousness would be a mystery demanding its explanation. It could be conceived of as no sport or casual result of a rare combination of circumstances. It is rather that which constitutes the main thread of narrative in the account which we must give ourselves of things as experience reveals them. How then are we to understand it? What are the underlying springs of movement? To answer this question we must first enquire into the causation of mind and its growth. We have treated mind throughout as a true cause. In the last analysis is it so, or is it at bottom an epi-phenomenon? On the answer to this question must depend our interpretation, and, therefore, our view of the future of the evolution that has been described. For on the one interpretation mind is a power that is constantly growing, and that has in the principle of harmony the vital seed of continuous expansion. On the other it is the superficial result of an adjustment of forces intrinsically indifferent to its growth or decay.

But further, even if mind is a true cause, the mind, whose development we have traced, is only one cause-among others. It strives with indifferent and even brutish conditions. It grows and increases its mastery over these conditions. But it has to fight every inch of its way. It can make no pretension to be the Absolute or the Unconditioned. It is a process within Reality, conditioned closely by other elements of Reality. Can we obtain any light as 
to its relation to these conditions, so as to learn something of the origin and meaning of the development which we have seen in process? This is to ask whether we can get at the causes of the process. There are two ways of approaching this question. One is to investigate the process itself. This we have done as far as we could. The other is to investigate the nature of Reality as a whole. This we might attempt through a synthesis of experience, but here our difficulty is that it is just the incompleteness of experience that has forced the present question upon us. Our only resource is to consider whether we have any general principles which, notwithstanding the limitations of our experience, we can affirm with confidence of Reality in general, and which will help us in the present problem. Now this, it may be said, is nothing but an invitation to enter upon the bog of speculation. The nature of Reality is not to be determined by an analysis of conceptions, but by a synthesis of experience, and when that synthesis fails we can go no further. As against an analysis divorced from experience this criticism has force. But it may be that an analysis of fundamental conceptions, for example, of the causal process, is just the link that is required to complete a synthesis of experience. It may be possible to co-ordinate analytic enquiry and empirical results. In the special sciences abstract principles, when tested by concrete experience, make good hypotheses, and the same method may be applicable to the science which deals with Reality as a whole. If, that is to say, analysis of first principles leads to a certain conception of Reality, and if this conception coincides with that which the widest obtainable synthesis of experience suggests, we have something more solid than a metaphysical speculation, and of wider applicability than an empirical generalisation. I shall endeavour in the end to show that such a correspondence of analysis with experience can, in fact, be found, and that the resulting conception of Reality has more than a merely speculative value.

We have then two questions to face. The first is whether mind, as we know it in the living being, is a true 
294 DEVELOPMENT AND PURPOSE CHAP. III

cause or only an epi-phenomenon. The second-since at the utmost we do not suppose mind in living beings to be the sole cause of its own growth-is whether we can find out anything about the causes of this growth in general. Both questions concern causation, and suggest the necessity of a preliminary examination of the causal concept. 


\section{CHAPTER IV}

\section{MECHANISM AND TELEOLOGY}

I. ON the surface, when we seek to explain any fact or object of experience, we seem to ask sometimes one, sometimes another of two very different questions. The 'why' of a thing means either its cause or its purpose. Every explanation falls within one or other or both of these categories, of which the one is known as the category of mechanism, the other as that of teleology. Let us consider the distinguishing characteristics of these two categories, and to do so let us take a case where either category is equally applicable. If we ask, for example, the explanation of the motion of a given wheel or lever in a machine, the answer may take two forms. First, it may be pointed out that the lever performs a specific function in the machine, it opens and closes a valve, let us say, which admits steam to a cylinder, and thereby governs the working of the engine. This is a teleological explanation, and that it is prima facie admissible in the present case nobody doubts. Let us see to what questions we are led if we pursue the enquiry on this side, if, that is, we follow the teleological line. We shall see that this line divides into two branches. On the one hand it leads us on into an enquiry into the mechanism of the engine as a whole. Our particular lever was, say, the eccentric that works a slide valve. Having ascertained how the slide valve moves, alternately opening and covering three apertures, we proceed next to the enquiry what this alternate process affects, and thereby to the structure of the cylinder, the piston and its connections on the one hand, and the steam pipe, boiler 
and furnace on the other. That is to say, we come to understand our original lever, the fact or part from which we started, as part of an arrangement fixed there to work in with the rest of the arrangement, determined, we may say, by the arrangement as a whole. This line of investigation then, as we follow it out, leads to an interpretation, as complete as we can make it, of a system of interacting parts. On the other hand, the system as a whole is governed by a certain purpose, which it serves in its completeness, and only in its completeness. The engine is to draw a train, propel a ship, drive a cotton mill or whatever it may be. The second line of enquiry which teleological investigation opens up is into the nature or value of this purpose, and here again the immediate purpose may be part of a system of values. It may conceivably be an end in itself, or it may be a means to an end, or perhaps a means to more than one end. Thus the immediate purpose of the locomotive is to convey passengers and goods. In a more ultimate sense it is, from one point of view, to facilitate the business or pleasure of the public, from another to assist in earning dividends for the company. Whatever it be, the enquiry into the why of the thing, pursued along this line, must lead us to something, simple or complex, to which as such, and not merely as a means to something else, we can attach definite value. It is, in fact, this last point that is essential to teleological explanation.

While a conception of value is capable of lighting up an arrangement of indefinitely great complexity, it by no means follows that complexity of arrangement is necessary to the useful application of the idea of value. On the contrary, many actions of extreme simplicity have a teleological explanation in the immediate pleasure attending on them. We walk or swim or look at a view for the pleasure of walking, looking or swimming, and though the biologist may tell us that there is in these things an ulterior value, we feel this to be in a sense a supererogatory explanation. It gives a reason why we should feel pleasure in the kind of exercise in question, but for the exercise itself the pleasure alone is a simple and sufficient reason. Teleological explanation is as such the reference of a fact, an object, a 
process to some end of value which it subserves. This value may, though it need not necessarily, appertain directly or indirectly to the working of some complex system as a whole, and if so, it is the point of departure from which the entire arrangement is to be understood, every element in the system being determined by the part it plays in interaction with others in contributing to the general purpose.

So far then as a system has value, every part in it is determined by relation to other parts. This determination has a very precise sense. Quite literally, this particular eccentric is to be seen at work in this machine, was cast and made true and pivoted on to its shaft because there is a slide valve to be moved to and fro and a cylinder with a piston moving back and forth. A modification in one of these parts may produce corresponding modifications. A different type of valve may require a different gearing, and a turbine postulates a wholly different arrangement. In a word, teleologically considered, the parts of an arrangement are not indifferent to each other. They are brought into existence, they are put together, they perform each its proper function as parts of a totality schemed on certain lines to produce a given result. In this totality each bit exists $(a)$ because the whole has an end of value to subserve, $(b)$ because the residue of the plan requires precisely this bit to be added to make up the whole. The absence or essential change of this bit must then involve either a modification of the whole, i.e. a change in, if not the total disruption of, its peculiar value, or a corresponding modification of the residual plan. It is in this sense that in any teleological arrangement the parts interact and involve one another.

2. So far the purpose of the lever, eccentric, or whatever the mechanism be. We have now to observe, secondly, that to the 'why' of the process it is equally possible to give an answer on quite different lines. This lever has a reciprocating motion at the one end and an elliptical motion at the other, because it is screwed into an eccentric and pivoted to a reciprocating rod. The eccentric in turn is rotated by an 
axle, and so forth. To ask the 'why' on these lines is to trace, not the purpose or function but the 'cause,' and to trace back the line of causation is to follow out the category of mechanism. The word is indeed something of a misnomer, since few arrangements, if any, are so clearly teleological in their entire nature as a machine. None the less, usage seems, in philosophical nomenclature, to have assigned the term mechanism for the category of explanation from which purpose is excluded. Let us endeavour, following the lines of this category, to compare the results point by point with the former. The first point that will strike us is what we may call the indifference of mechanism. When we asked why, i.e. with what purpose, the lever moved, the answer implicated the rest of the machinery and ultimately the purpose which its working subserved. When we ask why, i.e. for what cause, the lever moves, the answer is immediately, it is attached to an eccentric, and the eccentric rotates on an axle and the axle is turned by a crank and so forth. This line of explanation also in one sense takes in the whole machine bit by bit, but after a different fashion. The mechanical causation of any part of the process proceeds without regard to the surroundings and without respect to the purpose or value of the whole. A given stroke of the lever takes place because the eccentric makes a turn or a portion of a turn. It does not matter whether the engine is working or whether the axle is turned by hand. It does not matter whether the lever is connected with the slide valve or broken off by a sudden accident, it does not matter whether the slide valve, being moved, will admit the steam in the ordinary course, or whether, owing to a dislocation, the motion is futile or harmful. These things will affect the permanent working of the lever. It will not continue to act if the machinery is deranged. But if we fix our minds on a given stroke and ask for its cause, it is a given turn of a particular axle. Given the physical connections, this causal relation will hold, and will hold without regard to any concomitant circumstances or subsequent effects whatever. If we were to analyse it down further into its elements, considering the strains and stresses on rivet and bar, the impacts, the 
pulls, the forces and resultants, initial and later velocities and so forth, we should in each case seek for a relation more and more atomistic, as it were, and self-contained in character. With more and more certainty as we made our analysis precise, we should be able to lay down without limitation, that given the cause the effect must follow, let all concomitant circumstances be as they might be. Thus, while the category of teleology leads us to conceive of each object, event or process as implicated with concomitant processes of some arrangement, the category of mechanism leads us to consider it as dependent upon, following along its own peculiar line of causation, which, if accurately stated and fully known, holds its own no matter what the accompanying circumstances may be. So even if in tracing the cause of a given motion of our lever, we are driven back through axle and crank to take account of the entire machinery, yet all this comes into the account only as a part of the history of the movement studied. Each bit of the machine might be destroyed the moment it had performed that particular motion upon which the process that we are explaining depends. Provided that its motion was complete, our process will go through. The destruction which renders it teleologically absurd does not mechanically affect it. So in fact in a machine which is in some way out of gear, the mechanical continuance of some displaced process which is no longer fulfilling its true function may continue indefinitely, perhaps to the destruction of the machine. In fact, as the mechanical operation of cause and effect is indifferent to concomitants, so a fortiori it is indifferent to results or to values. For the cause of a thing we look always to the past. More strictly, we seek to produce the effect which we desire to explain without break of continuity into the past, and it is this self-contained continuous strand of active being which, when for clearness we analyse it into an earlier and later, we call cause and effect. In tracing such a self-determined strand in time, we never think of the earlier as determined or conditioned by the later, for this would be to think of the existent as determined by what does not exist. We think of that which exists now as giving rise by continuous 
transition to that which exists later, as, in fact, becoming it, of its own nature and without the aid of any adventitious concomitants. This unconditional continuous becoming is the ideal to which mechanical explanation tends, and this once again is in direct opposition to the teleological conception, in accordance with which all the elements and constituent processes of an arrangement are indefinitely modifiable, and are in fact so modified as best to ensure the working out of a purpose which is subsequent to their action. Under the teleological category, in fact, it looks at least on the surface as though the future goes to determine the present.

Whether this first impression of teleological determination can hold in the end, we shall consider further at a later stage. We have first to point out that in our surface view both mechanism and teleology are together necessary for the full explanation of our lever. For merely to analyse the law of the lever's action is not to show how the lever comes to be where it is, while if we extend the 'mechanical' explanation so as to include the whole story of its formation and insertion into the machine, we shall have to take account of the engineer's mind and of the purpose which the machine is to serve. That is to say, the 'full' mechanical explanation will involve the teleological. But conversely, the teleological involves the mechanical. The precise function to be fulfilled by the lever is indeed prescribed by the purpose of the machine and the general arrangement, but the way in which this particular lever performs that function is to be understood only by studying its peculiar reactions. Mechanical actions are the units out of which the working process is constructed, just as the physical bolts and cogs are the units of which the arrangement, as a material structure, is built up. The full explanation of our piece of mechanism then must include both the analysis of its own operation and a statement of the teleological system in which it forms a part.

3. We have seen that our mechanical explanation is forced ultimately to take account of the constructive purpose of the engineer, in order to explain how the lever came to be 
where it is. It will repay us to examine further into this necessity. It does not arise immediately. We could, for example, take the action of the lever at a given point in its stroke and connect it with the whole configuration of the machine at the same moment. We could then, on purely mechanical principles, trace back this configuration to the preceding configuration and so on. It is only when we ask about the initial step, how this particular machine came into being and why it was set to work, that we are forced outside the mechanism itself to human hands and human minds controlling the whole. The reason why we are thus driven outside is that the machine does not explain itself. Its parts have, apart from their purpose, no intrinsic connection with one another. We can see that this rod works in that socket and is made to fit it, but we see at the same time that it does so only because it is made. The socket, as a piece of metal, does not intrinsically necessitate a rod working through it, nor the rod a socket. They are, as it is sometimes put, quite external, or, in our previous phrase, they are indifferent to one another, and it requires an outside force, the hand of a workman and the brain of an engineer to bring them together. To find that which in physical fact brought them into connection we must go to the purpose, which thus figures as the unitary principle connecting things otherwise alien. Conversely, as long as we disregard the purpose of a mechanical arrangement or configuration we can explain it only by showing bit by bit how each element of it grows out of the corresponding element of a previous configuration. There can be no mechanical explanation of a configuration as such, but the reference must always be to an antecedent configuration and so ad infinitum.

Now in the case of the machine, this reference drives us in the end to an external agent. This is due to the indifference of the parts. One lever does not create another to work with it, nor depend for its growth upon another. Suppose, however, we were to find any arrangement in which externality were overcome, in which the growth and action of the parts were, in fact, mutually dependent, the case would be different. We might then conceive an 
internal principle of unity, and though we should in the end have to enquire into the origin of this principle, it would not be for the sake of unifying the elements of our arrangement. That unity would now be involved as a characteristic of the several elements themselves. It would be intrinsic or organic. Such a principle constitutes what we call an 'organic unity,' and an arrangement where on the surface we appear to find it we speak of as an organism. Whether, indeed, the physical organism which we naturally think of as the type of such a structure does indeed conform to the conception or is rather to be regarded as a mechanism of exceedingly cunning contrivance, is an empirical question on which scientific investigators do not agree. Our business, for the moment, is to clear up the conception of organic unity and to put our finger on the points which distinguish it from that of a machine. Whether the conception that results is a mere figment of the imagination, whether it is realised in plant and animal life, or elsewhere, or nowhere, are further questions. What is certain is that many phenomena of plant and animal life have, rightly or wrongly, gone to generate the conception, and we may freely refer to these without asking in each case whether the interpretation which has led to the organic view is the ultimate truth. On this a word may be said later. Our immediate purpose is not to justify a particular application of an idea, but to explain the idea itself.

4. The living organism then is, from one point of view, comparable to a machine which, while performing various operations on the external environment has, both in these operations and in its internal changes, the maintenance of its own activity for its object. Whether, indeed, purpose as such is properly to be ascribed to organic activity is one of the questions to be determined, but it may suffice to note for the moment that the more definitely we conceive of the working of the organism as mechanical the more readily we are led to set a purpose outside of the mechanism as the controlling principle of the arrangement of its constituent parts and processes. But letting this point pass for the moment, what we have first to observe is the relation 
of these constituents to one another. Mechanically, the organism may be conceived, like any other machine, as essentially an arrangement for the transformation of energy. Thus the animal organism takes up energy in the form of food on the one hand and of oxygen on the other. For each process of absorption it has its appropriate mechanism, the alimentary and the respiratory organs. Next, it has to distribute what it absorbs by means of its circulatory system, and thereby to nourish nerve and muscle tissues wherein the potential energy of the foodstuffs is converted into energy of motion, so directed through the nervous control as to secure fresh supplies of energy and at the same time maintain at the right point, neither too high nor too low, the temperature at which this persistent activity of change or metabolism can go on. Finally, the waste products which result have to be eliminated, for which purpose the circulatory, respiratory and alimentary systems, together with other special organs as the kidneys, again come into play. Of the reproductive functions we need not here take account. It is enough to recall in rough-and-ready way the picture familiar to common sense and elaborated in detail by physiology of the individual organism as a going concern in which a total process, the metabolism or life of the organism, is maintained by the co-operation of a series of parts, the final result of which, when it comes full circle, is just self-maintenance.

Now, at any rate, as long as we ask no questions about origins there is nothing here to differentiate the organism from the well-compacted machine. In fact, the physiologist in seeking explanations of the life process moves habitually, and often with brilliant success, along the lines of mechanical explanation. Thus he can follow the circulation of the blood by conceiving the heart as a force pump and the arteries and veins as a connected system of elastic tubes. He may begin with the left ventricle, and show how the blood is expelled by a strong contraction which, closing the valves that lead back into the auricle, open those of the aorta. He will show that this new tide of blood, aided, moreover, by the contraction of the aorta itself, will propagate a pulse through the arterial system and force the 
whole blood stream along throughout the tissues. He will thus follow it through the branching arteries into the capillaries, observe its interchange of substance with the cells which it bathes, and thus account for its emergence from the capillaries into the veins in the changed character of venous blood. In the same way he will follow it back to the right ventricle and thence through the pulmonary circulation where it is restored to its arterial character, to the left auricle, and by the valvular mechanism to the left ventricle from which he started. Here the essential features are mechanical or chemical, and for our purpose we may assume that the chemical is, by methods which year by year come more clearly into view, to be reduced to the mechanical. Nor need we stay to enquire into certain points of the explanation which might present some difficulty to the mechanical view, by asking, for example, how far the interchange of substance, which is the essential point in the whole function, can be squared with the physical laws of diffusion, or whether the behaviour of the arteries can be wholly understood on the analogy of elastic tubes. We may better attend to points which, not by their obscurity but their clarity, emphasize the specific character of an organism. This circulatory process, for example, does not work with even regularity. If the body is thrown into violent muscular exertion the metabolism of the muscular tissue is proportionately heightened in order to supply the requisite amount of kinetic energy. This augmentation requires in turn an increased supply of oxygen while it produces a surplusage of oxidised brokendown proteids which have to be eliminated if the muscle is to continue to do its work. These requirements can only be met by an increase in the blood-stream, both to bring up the oxygen and to remove the waste, and in the healthy organism this supply is forthcoming through an acceleration of the heart and a dilatation of the arteries, which dilatation is, moreover, localised if a particular set of muscles have alone to be supplied. At the same time, respiration is quickened, so that the blood is more rapidly oxidised. The action of the heart and arteries then appears to be determined by the function which they have to perform, 
and the respiratory system responds in sympathy. Now this, on the surface, throws them into strong contrast with the parts of a machine, each of which, as we saw, must do what it does irrespective of the working of the rest. But it will hardly be supposed that the anti-mechanical view is to win so cheap a victory as this. We have to ask how the quickening of the heart and dilatation of the arteries is effected, and here at once a further and special mechanism is found. Heart, arteries and lungs are alike under the control or partial control of nerves, and these nerves are affected by the condition of the blood. Thus the respiratory nerves are traceable to a centre in the medulla, the action of which responds accurately to the degree in which the blood is oxidised. If the supply of oxygen falls off the de-oxidised blood acts as a stimulus on the centre, heightens the activity of the nerves which supply the respiratory muscles and so quickens respiration. As the normal state of oxidation is regained the stimulus falls off and breathing resumes its normal course. A similar selfbalancing machinery can be indicated for the other processes concerned.

5. In these explanations, it is true, the phenomena of nerve stimulus and reaction have to be employed. These are peculiar to the living organism and have not as yet been reduced either to mechanical or chemical terms. But on this point once more we lay no stress. We take them provisionally as mechanical in the sense that, given the musclenerve arrangement, stimulus A will invariably produce reaction $\alpha$, and stimulus $\mathrm{B}$ reaction $\beta$, with no regard to results or concomitant circumstances. Once again we concentrate attention on the working of the process as a whole. What we find is that the circulatory and respiratory organs on the one hand, and the skeletal muscles which move the limbs on the other, are not, as in the ordinary machine, mutually 'indifferent.' The working of each is intimately affected by the working of the remainder. Not merely are they arranged once for all so that by a regular rotation each supplies or supplements the other, but on a far more complex plan they are arranged so that variations of their 
activity dovetail in with one another and maintain an equilibrium among an ever-moving set of forces. Whether through a subtle mechanism or otherwise, the result is reached that the several parts do not act independently but in mutual relation. Mechanical 'indifference' is replaced by organic 'consensus.' Bringing the two opposed terms down to their last analysis, so far as it is as yet before us, we may say that two parts $a$ and $b$ of a whole are mechanically related when the operation of each is uniformly determined by its own structure alone; they are organically related when the operation of $a$ is itself affected by the effect which it has upon $b$ and vice versa.

We shall have to return upon this definition very shortly and to ask not only whether it is satisfactory in itself, but whether it serves adequately to distinguish a living organism from a machine. It will be well, first, to remark most briefly that the consensus which we have recognised affects not only the daily and hourly working of the organism, but its structural growth. Just as between two functions so between two structures, modification is met with modification. Within the limits of organic adaptability alterations of conditions are met by a responsive growth of structure which, whether with or without some general modification of type, enables the life process to be maintained. In the first place, the normal development of the entire organism, and of every part of it considered internally, is a correlated development. Starting, as it does, with the division of a single cell, apparently through the development of certain centres and radiant lines of tension, the very first stage presents us with two cells determined in size, character, contents and position by the mutual relations, the relative tendencies of different portions of the substance of the mother-cell to hold together or to split. Each stage of growth involves essentially similar processes of cell division, and thus the gradual differentiation of parts out of a relatively simple and homogeneous structure is a process in which, take it where we will, each new element is a differentiation involving its complement. But further, the lines of differentiation are not absolutely predetermined for each individual embryo. On the contrary, 
experiment has shown that the mutilation of an embryo at an early stage may induce a far-reaching readjustment. In some cases, for example, the daughter-cell produced by the first division of the fertilised ovum, which normally develops into one half of the embryo may, upon the artificial removal of its fellow, be made to do duty for the whole. It may develop not as usual into the half but into the entire organism. Similarly, and on more familiar lines, any deviation of one tissue from the normal will involve a response on the part of other tissues. A curvature of the backbone alters the whole upper part of the figure, and a number of tissues must accommodate their shape accordingly. The cells of the skin, for example, multiply only so far as is necessary to cover the dwarfed skeleton. Similarly, in the adult organism, lesions and abnormalities of all kinds are met with special growths of suitable tissue. Constant use of the hands does not wear away their substance with friction as the surface of an inanimate object would be worn away. It stimulates the production of horny substance by the cells of the epidermis, and the result is a hand not less but better fitted for its work. The athlete's heart braces itself to its excessive labour by thickening its muscle. Even the bony tissues adapt themselves to special strains and alter their structure to meet new conditions. 'If the bone is broken and heals out of the straight, the plates of the spongy tissue become rearranged, so as to lie in the new direction of greatest tension and pressure ; thus they can adapt themselves to changed circumstances.' ${ }^{1}$ The elementary truth of practical life, that the living being grows and flourishes through and by means of its difficulties, dangers and toils, rests on this general reactive elasticity of the organism, that is, on the capacity of each part to adapt itself with structure and function to the needs of the whole.

6. Thus, alike in the growth, modification, and activity of vital structure, we see that close interdependence of parts, or what is the same thing, that adjustment of part to whole which our definition of organic unity required. The ${ }^{1}$ Weismann, Romanes Lecture, p. 15. 
question has now to be asked whether this interdependence may not, after all, be conceived in mechanical terms. May we not, that is to say, contemplate an arrangement, call it of organs, cells, molecules, or, if preferred, of forces such as (a) in response to normal stimuli will run a certain prescribed course, as a wound clock gradually runs down marking the hours the while, and (b) by special and highly intricate combinations will provide, within limits, for certain deviations from the normal. The nature of the provision may be set forth in this wise. Let us imagine elements A, B, C ... functioning normally along lines which we may distinguish as $\mathrm{A} \alpha, \mathrm{B} \beta, \mathrm{C} \gamma$. Then it must be prearranged that a change $\alpha^{\prime}$ affecting A produces a corresponding change $\beta^{\prime}$ in $\mathrm{B}$, and this again produces $\gamma^{\prime}$ in $\mathrm{C}$. The system $\mathrm{A} \alpha^{\prime}$, $\mathrm{B} \beta$ ', $\boldsymbol{C}^{\prime}$ ', we must suppose, will 'work,' that is, it will be able to maintain itself as a system just as the normal $A \alpha$, $\mathrm{B} \beta, \mathrm{C} \gamma$, can do. The simplest case, in fact, will be that in which the effect $\beta^{\prime}$ is such as to react upon $\alpha^{\prime}$ and tend to reduce its divagation from the standard $a$. This is the case, for example, with the repair of tissue or with the adjustment of the balance of oxidation in the example which we took. Somewhat more complex but still intelligible enough is the case where the organic structure is in some measure modified, but without losing its recognisable identity or interrupting its life process. In this case $\beta^{\prime}$ and $\gamma^{\prime}$ do not tend to neutralise $\alpha^{\prime}$, but rather to complement it. They are the modifications in $\beta$ and $\gamma$ required by the change in $\alpha$ in order to maintain the moving equilibrium. This is the case of the broken bone set crooked, which, to maintain its function as a whole, modifies the lie of its component parts so as to meet the new lines of strain.

Some such compensatory arrangements are undoubtedly observable in machines. The 'governor' of a common steam engine, for example, is a device whereby excess of speed, due to a sudden diminution of resistance, corrects itself by closing the throttle valve and diminishing the supply of steam. The 'compensating pendulum' maintains the resultant length of the pendulum unaffected or almost unaffected by changes of temperature. The very change which disturbs the balance in one direction is made 
to call into being a process which redresses the balance. More generally, in any self-acting machine, it may be contended that as soon as we consider its permanent operation, there is not that 'indifference' of parts which our definition postulated. Thus in our own example, though any given movement of the eccentric follows 'mechanically' on the turn of the axle, no matter what is happening to the rest of the machine, yet if we look at the normal working of the mechanism as a whole and consider the conditions on which the recurrence of this particular motion rests, must we not, after all, admit that it is precisely its relation to the remainder, the fact that it is connected up with a steam cylinder and its appurtenances that keeps it in being? Must we not say that as truly as in the living plant or animal, the working of the slide valve gear is determined not wholly by its internal structure but by the effect it has on the remainder of the arrangement?

If we admit the cogency of this argument we shall be forced to recognise in such an arrangement as a self-acting machine something of the organic character, and for this recognition, in fact, the result of our discussion will in the end provide some justification. The machine, however, remains in essence a machine, partly because it does not grow but is made, i.e. the organic character disappears entirely when we consider its genesis, partly, and this is the point to be emphasized for the moment, because the organic relationship is here, beyond doubt, secondary. It is the result of operations of part on part which, however cunningly devised to combine, to maintain one another, and even to regulate and compensate for divagations, operate from moment to moment, each in accordance with the laws of its structure, directly affecting and affected by that only which is in immediate continuity with it. Now the fundamental question for the validity of the organic concept is whether there is any structure where the action of each element appears to be determined by its place in the whole which cannot be reduced to a mechanism after this fashion. The organic concept seems to be something different from the mechanical, but if so what is the difference and how can it be explained? 
7. Let us, as before, suppose a living being with parts or organs $\mathrm{A}, \mathrm{B}, \mathrm{C} \ldots$ and normal life processes $\mathrm{A} \alpha, \mathrm{B} \beta, \mathrm{C}_{\gamma}$, and let us take the case where any large or prolonged deviation from $A \alpha$ is fatal to life. Then we shall find that modifications such as $\alpha^{\prime}$ set up deviations $\beta^{\prime}, \gamma^{\prime}$, which tend to restore $\alpha$. If we suppose that the modifications $\beta^{\prime}, \gamma^{\prime}$ are set going mechanically by $\alpha^{\prime}$, we must attribute them to a pre-determined mechanical arrangement. The characteristics of this arrangement are that it acts as a whole, maintaining as a whole a life process admitting of certain limited deviations in all directions from a common central type. Every elementary process sets up other processes which react on it, either (I) in such a way as to maintain it, carry it through and perhaps prepare for its repetition if it is a normal incident of the vital whole, or (2) so as to modify and thereby convert it into a process compatible with the whole. The organic whole may thus be conceived as shaping or determining its constituents as much as it is determined or constituted by them. Now, so far, we have conceived this relation mechanically. That is to say, we have conceived each process as beginning independently of the remainder, whether through environmental stimulus or internal changes of structure, and we have conceived an arrangement such as we have actually noted in the case of some machines, whereby such a process sets up others which, finally, through a series of causal links react upon it and shape it into conformity with requirements. The confessed machine will exhibit one or perhaps two such devices, affecting a particular part in a special way, through a series of connections. Let us suppose the reactions quickened and multiplied. The functions of any organ then come under the influence of others more speedily and more completely. Its action, not only in this respect or that, but in all respects calls forth reactions from the rest of the organism which affect it, maintaining, annulling or controlling as the case may be ; and the reaction is more and more speedy and direct. Thus the time and the sphere of the independent action of any part are conceived to be progressively shortened. In the limit both are zero, and the part does not for any time or in any respect act in 
independence of the whole, but its reaction, say, to any given stimulus is, from the first, determined by its position as a constituent of the whole. This appears to be what is properly meant by an organic as distinct from a mechanical process, and if this is so, organic unity appears as the limit of a mechanical adjustment of interacting parts to the maintenance of some common process or the furtherance of some common end. In proportion as mechanical adjustment becomes comprehensive and immediate it approaches the organic character. It could reach it only at the point where the succession of cause and effect, as between part and part, merges into the simultaneity of a consentaneous whole. A whole in which the reaction of each part not merely sets up further reactions which return upon it, but is itself determined by its bearing on the whole, is a true organic unity.

But how is this determination effected? In the whole $A, B, C \ldots$ the reaction of any element is to be determined by the requirements of the whole. It has to be, let us say $\alpha$. Now as long as we looked on A, B, C as a mechanical system, we could see a method by which, notwithstanding variation of circumstances, this result might be brought about. We conceived a quick series of interactions whereby A giving the reaction to $a^{\prime}$ affected $\mathrm{B}$, which by the reaction $\beta^{\prime}$ reflected on $\alpha^{\prime}$ and reduced it to $\alpha$. We conceived two separately centred but rapidly interweaving processes, and we could increase the rapidity and closeness of interaction at pleasure. As we do so the action of each part is more and more closely determined by the consequent action of the residue. At the limit it is so determined from the first, i.e. the action of the part is as such conditioned by actions of the residue which it itself sets up. The limiting, correcting, activity of this residue is now operative in the actions of the part itself from the first. But this is as much as to say that the action of any part of an organic whole is action conditioned by its result, i.e. is teleological.

Now it may be said that the action of the part is not determined by the reactions of the remainder, but that the part is such that under any given circumstances its action will be adapted to the requirements of the organism. In any 
concrete case it may be so. That is a question of evidence bearing on the history and behaviour of the particular structure. But we are here concerned with the concept of the organic and are asking ourselves what it is that we arrive at, if we imagine the action of one part corrected or modified by the reaction of others upon that action, and if we imagine the time of this reaction shortened till it becomes zero. The answer then is that we must now imagine the action of the part to be itself determined by the reactions which it will produce. That is to say, that if we conceive an organism as a structure whose parts are determined in their actions by their relation to the whole, we have to conceive it as one whose actions are determined by their results.

Further, what is true of one part of an organic whole qua organic is true of all the rest. This implies that the process which each part follows in accordance with its intrinsic conditions is so correlated with the processes of the other parts as to maintain them and carry them through, while if the conditions of any part are such as to maintain it unchanged, wholly or in certain respects, the action of other parts is similarly conditioned by their effect in maintaining that part unchanged wholly or in those respects. A system of parts so related that each is thus conditioned in its action by its effect in maintaining a residue is a harmonious system. An organic unity then is harmonic and teleological.

If that is so, the organic process proper rests on a causation that differs in kind from the mechanical. By perfection of predetermined arrangement the mechanical may acquire more and more of organic and purposive character, but in the limit, where the correlation is complete, it passes over into the region at once of organicity and of purpose.

That is to say, if we start with a system of mutually determining processes which yield a certain result, and if we press the mutual determination closer and closer to the point at which it becomes Harmony, our conception of the process must undergo a parallel change, and from being the mechanical effect of a contrivance arranged to secure the result, must be regarded as a process guided by the 
result itself or determined by its own character as yielding that result. Observe, further, that if the action of each part is conditioned by its results, the result is equally conditioned by that action, and thus there is in teleological action an organic relation, not merely between the contemporaneous activity of parts, but in the succession of phases. The causes together determine the effect, while it is its bearing on the effect that determines each of the causes.

8. To this account objections may be taken which we will examine without delay. But let us first observe the result to which we have been led. To explain an event or a process, we have found, may mean two things. First, it may be to find for it an antecedent event or process which passes into it, proceeding without any reference to concomitant processes or events. This is the mechanical cause. Secondly, it may be to find for the event - and this will mean for the entire mechanical process which hangs together-an explanation of its existence here and now, a reason why it holds its place in this particular collocation. Explanation on these lines cannot be mechanical, but may be teleological, i.e. may be effected by referring the collocation as a whole to a purpose. But it has appeared that there are two forms in which purpose may operate. If the parts of the configuration bear no necessary relation to one another, the configuration, if it serves a purpose at all, must be regarded as something imposed on them by a purposive mind. We have a machine with an intelligent creator as its antecedent cause standing outside it. If, on the other hand, the parts are each such as to contribute to the maintenance and growth of the whole, while, conversely, they could only come into being in relation to each other, we have an organic whole. The definition of the organic whole would then be, that in it each part is determined in its existence and its behaviour by the effect of its action on the whole. The organic whole would then, as such, be teleological, but the operative purpose would be within it, dominating each part. Such a whole would correspond to the ethical conception of a harmonious system. Thus, to explain a collocation otherwise than by referring it to an antecedent collocation is to 
refer it to a cause which cannot be mechanical but may be a purpose, either operating upon it from without, or operating within and pervading all its parts. In either case the explanation in this sense is teleological.

9. But we have now to take account of two objections which may be raised against this conception of an organic whole and of teleological explanation in general.

It may be said, first, that this determination of a process by a relation to its result is utterly impossible, and, secondly, that every apparent case of such determination may be explained by the structure which has come into existence adapted, in accordance with mechanical laws, to yield the required result. On the last point we may remark that the question is not how an organic structure comes into existence, but what it is and how it acts, and if a thing so acts as to be determined by the relation of its function to its effect, it is acting teleologically. The question then is whether such action is possible. If not, then every apparent case of it must be resolved into a mechanical adjustment which simulates teleology. If yes, then we may approach any case without prejudice and decide whether it is one of genuine teleology by an inductive determination of the actual causation at work within it. We have then, first, to ask whether there is any possible sense in which a process can be conceived as determined by relation to its result. As a mode of speech we all understand what it means. If I hail a cab to take me to the station, catch the down train and get home in good time for dinner, the dinner and all that appertains thereto and the hour for which it is fixed, may be spoken of as the determining or governing fact in my whole procedure. But can this for a moment be regarded as an ultimate analysis? At the time when $I$ hailed the cab the dinner is non-existent. Does the non-existent cause the existent? It may be that in the eternal scheme of things the dinner is fixed, and I might, though by a somewhat desperate device, take what is to be as equally real for causal purposes with what is now. But even granting so much, how could we deal with the purpose which is not 
realised and the dinner which does not come off? If the cab breaks down or I break my leg in getting out of the train, the dinner which seemed to have determined my behaviour was not, after all, written down for me in the beginning as a part of the scheme of things. Not only was it non-existent at the time of its alleged causal efficacy, but it never came into existence at all. It had no place in that framework of things in which it was called on by our teleological category to play an unassuming but not irresponsible part.

These difficulties, we shall surely be told, arise only from a childlike acceptance of ordinary ways of speech. The future is in no sense a true determinant of the present. In a causal relation the antecedent is always an existent, and in a teleological system which the ultimate result appears to dominate, the true controller is a mind animated by an idea which does indeed project itself into the future and guides events in accordance with the lines of projection, but as an operating force in the disposal of events is an ever-present agent, acting by its presence alone. It was the working of a mind as an external agent which we assumed always as the explanation of the arrangement of parts in a confessed machine, and if a mind can make a permanent arrangement which by regular action can secure a certain result, so with more plasticity and closer attention to detail it can guide systematic operations which will be able to deal adequately with the shifting requirements, the changes and chances of more complex mutual conditions, and select always out of many possibilities the actions best adapted to the furtherance of a particular end. In short, on this view where there is systematic co-ordination apparently dominated by an end, there is in reality a mind inspired by a purpose which is the present operating force, and if we are right in conceiving organic adaptation as determined by its results, that must mean that we conceive the living organism as so far endowed with intelligence. But there is no such thing as determination by the future or by relation to the future. A formed purpose may be a cause, but it is also an effect. It is something that grew out of the past and acts now just as any mechanical con- 
figuration arose out of the past and acts now. The past wholly determines the future and is in no sense determined by it.

The objection to this account is not that it identifies teleological action with the action of mind. On the contrary, this identification is at least probable and may be provisionally assumed, with the consequence for us that all truly organic action is of mental character. Nor is it that it insists on the present existence of the cause at the moment of its operation. This existence we must assume. The objection is that the account gives no analysis of that relation to the future which it admits in the activity of Mind. It sets out to exclude the future from causal operation, yet it can explain the action of Mind only by speaking of a projection into the future. Thus it leaves a contradiction standing which we must resolve if we are to understand teleology and the precise point of its distinction from mechanism. We have still to ask, then, can anything causal, be it mind or be it what it may, be conceived as in any literal sense determined by relation to its result? The point is fundamental, because if there exists anything of this kind, then also there exists a mode of causation differing fundamentally from the mechanical, and if not, mental, purposive, operation is itself ultimately mechanical.

Mechanical causation is a continuous process in which each phase is determined wholly by that out of which it issues and in nowise by that into which it will pass. It is this indifference to what is coming which is the real root of that indifference to concomitants which is the external feature distinguishing the mechanical from the organic. Thus, if a book be pushed along the table till it fall over the edge, the resultant motion before and after the critical point is wholly different, but the effect of the push as such, precisely the same. Before, it was compounded with two forces (the weight of the book and the support of the table) which were in equilibrium. Now the support of the table being withdrawn it is compounded only with the weight of the book, but is still recognisable to kinetics in the curve which the book describes. If the push were a purposive effort to bring the book to some point in the 
direction to which it tends, it would at the table's edge abruptly change its method, but regarded as such an effort it is futile. Water acted on by gravity falls into a pool and remains there. If, for a moment, we imagine the brute force of gravity to be in reality a desire to get to the centre of the earth, we might say, if the water had but the sense to hold itself up but a moment longer it might have gone over a ledge of rock and fallen many feet further. But in any mechanical tendency, however persistent, arrest even by one moment is fatal. There is no going round. Now this going round to get to a goal is precisely what we do find in the operations of conscious purpose, and it is this which justifies as a descriptive statement the formula that purposive action is determined by its end. Prima facie the matter is one of a straightforward application of inductive methods. Here is an action $\mathrm{A}$ which tends to an end $\alpha$. In varying circumstances $\mathrm{BC}, \mathrm{DE}$, the action $\mathrm{A}$ is performed and $a$ secured. Conversely, in circumstances FG, HK, A does not serve $\alpha$, but $\mathrm{L}$ does, and now $\mathrm{L}$ is performed. It is, prima facie, a sound induction that the tendency to produce $a$ is the cause of the action, and the inference is applicable to cases, e.g. of animal behaviour where there is neither internal consciousness of purpose nor language to tell us of purpose. But this raises the preliminary question whether it is possible that the tendency to the result should determine the act, and if so, in what sense. Now, if we look at any of the means used by an intelligent agent, be it a material tool fashioned for a purpose, or a course of action chosen with an object, there is a clear sense in which we may say that these owe their existence to the effects that they produce. The tool has been made, has been brought into existence by the agency of the intelligent artisan, because of its efficacy for his end. Not strictly the end itself, but the efficacy of the thing towards the end is quite literally a condition of its being. The same argument will apply to the performance of acts in a purposive series. Act or instrument owe their existence to something pre-existent, a purposeful intelligence, but the link is their causal efficacy. They are brought into being as the starting-points of certain trains of causation 
which are to be gathered together in the general purpose.

I0. So far there is little difficulty, because the mind in which we place the centre of the teleological system is conceived as standing outside the instrument and shaping it, operating upon it as any other existent cause may do. But this operative organising activity is not restricted to the external. Within the mind, so far as any given purpose possesses it, feelings, impulses and thoughts come under its moulding power. Even the emotional interest in the end itself is mastered and moderated if its excess interferes with the steady movement requisite to secure the aim. The purposive mind presents itself, in fact, as an organised system of elements-organised for the production of a common end. Whereas in a field of mechanical elements we have a set of forces subsisting side by side, each producing its own effect without regard to the rest, and unaffected by the resultant character of the whole process which their interaction forms, in the case of purpose we have a system of elements in which the part played by each is subdued to the requirements of the whole. In short, we have an organism. Just as the conception of organic unity led us up to that of purpose, so the analysis of purpose leads us back to organic unity. But in so doing it has brought to light the principle of organisation for which we were looking. The principle determining the relation of elements in the organic unity, that is to say, is the tendency of their combination towards a certain result.

But here we touch the centre of the problem. We have seen that this principle of determination has a clear and consistent meaning as long as it is applied to means, or even to psychic elements as a whole, so long as we could conceive the purpose as something acting upon and shaping them. But to conceive the purposive consciousness in this external fashion is to destroy its organic character. What holds of the means, however, will be seen on close analysis to apply also to the mind, which uses the means. The means come into existence, we agree, on account of their causal efficacy, as starting-points of certain lines or streams 
of causation. Now if we look at the purposive state as we know it in ourselves, we say familiarly that it is guided by an idea of the end and of the way and means thereto. This idea is a forward-looking something; its relation to the future, to what is to come out of it, is an integral part of its being. It is, we will not say, determined $a b$ extra, but constituted by this relation, this element of movement which it contains. But the forward-looking idea is not the whole of the purpose. The idea must interest, arouse feeling, dominate impulse. The purposive state is an impulse-idea, a conative state, an idee-force. It is forward looking, but more than that. It is forward moving.

But the direction of the forward movement is controlled, point by point, by the idea of what is to come qut of it. So far as the result is accurately forecast and on rational grounds, the future itself in its relation to the present is a true condition of the purposive state; for, were it going to be otherwise, the grounded forecast would be different, and the purpose would differ accordingly. The relation of present to future is then in a completely grounded teleology, not, indeed, the whole cause, but a condition of the act. But the actual course of the future may not be, and, of course, in human action is not, fully known. What is known is the tendency of the act, the result which it makes for, its causal efficacy. The purposive state of our experience is a process moving under the control of the idea of its own causal tendency. The tendency, that is, is the content of an idea which is an element in the purposive state, and a condition of its operation. Its tendency may thus be as truly said to be the condition of its operation as the actual course of the future is the condition of rationally grounded and adequately constructed purpose. Generically then a purpose may be defined as a cause conditioned in its operation by its own tendency. Its own causal efficacy, even more than in the case of the means, is the condition of its existence. It is essential to its constitution. Not the result as an event which may happen to-morrow, next year, perhaps never, but its own movement towards the result, the conational movement that it initiates and sustains, is integral and essential to its being. It is in this sense that 
the line of movement leading up to the end is essential to the existence of the purposive state itself, and thus, in a further sense, purposive activity is of organic character, for the movement towards the end depends upon, and is executed by, a process which depends on it. There is that mutual relation of origin and end which we found in our analysis of an organism holding in the interactions of part with part. The doing is determined by what is done.

This account of purpose is only set into clearer relief by the explanation which would reduce it to mechanical categories. The evolutionist will tell us that the biological reason why certain purposive tendencies exist in the living being is that they form the arrangement best fitted to secure certain results of value to the life of the species. Be it so. Then this shows that, however the purposive consciousness has come into being, its nature is so to organise things as to secure results, and that its efficacy in securing results is precisely the cause of its arising wherever it has arisen. It may be objected that an abstraction like causal efficacy can explain nothing. But, of course, the causal efficacy always has some concrete shape. It is the edge by which it will cut that determines the shape given to the tool. In the purpose it is an organisation of elements of thought and feeling, of physical acts and of external things that constitutes the efficacy of the action. The purposive state has historically come into being, because that sort of organisation does yield that sort of result. It is maintained in being by its own knowledge that it is tending in this direction.

I. I conclude that the main objection to teleological explanation is not sustained. There is an intelligible sense in which events or processes may be regarded as determined by their relation to results which are to come out of them in the future. This explanation may be applied to an event or series of events arising out of a purpose, but so far as the series is merely referred to a purpose that appears to stand outside it, the events seem to follow from it as a mechanical sequence. The explanation, however, can also be applied to the purpose itself, and when the originating purposive act is brought into the account, the whole system-the 
purpose, the train of events which it sets up and the ultimate end are seen as an organic whole, in which each element owes its existence to its relation to the remainder. Conversely, an organic whole is one which is determined by a purpose. The two forms of purpose discriminated above (p. 3 I 3) must in the last resort come down to one. Of course, it remains true that in a machine the configuration is imposed $a b$ extra on an indifferent material. But precisely so far as this is true, the existence of the material remains outside and unexplained by the purpose. All that falls within the purpose, the shaping and arrangement of parts, owes its existence to the purpose and contributes to it, and so forms an organic whole. Conversely, the bare existence of the material is a datum left unexplained by the purpose. So far then as there is explanation of collocations, it is by reference to a purpose which is also a principle of organic unity.

But all this only shows that the teleological concept is logically possible. It does not prove that it actually applies to anything in the real world. How are we to decide this question? There are two possible lines of approach. (I) First, we may continue to follow the logic of explanation. We have seen that, when we explain the existence of any thing or any process in a particular space and time relation to other things, we do so by assigning to it a certain place in a system. This raises the question of the explanation of the system itself, and here we saw two possibilities. The parts of a system might be mutually indifferent, in which case they must be brought together by some external agent, whose action could be explained if it served some purpose. Or the parts might necessitate one another, in which case, in the end, their existence and activity must be regarded as determined by their functions, i.e. by their tendency to produce certain results. In either case the explanation was teleological, and we have now seen further that teleological explanation is something intrinsically possible, self-consistent, and radically different from mechanical causation. Still our only positive result so far is to show that in two contrasted cases collocations can be explained by showing that they serve a purpose. We have 
not proved that there is no other possibility of explaining them, though we have as yet seen no other way. Nor have we determined what is perhaps a prior question, viz. whether collocations need any explanation at all beyond that which consists in reference to an antecedent collocation, and so ad infinitum. The answer to these questions will determine the range of teleological explanation, and will be attempted in the next chapter. But (2) meanwhile in certain cases there is another method of approach. In certain cases the intrinsic character of the process suggests a teleological explanation. Are we able by any sufficient evidence directly to confirm or refute the suggestion? The cases in question are the operation of our own minds, and the action of living beings generally. Can we establish purposive action in these cases? If we are satisfied that purposive action is possible, this will be in effect to ask whether we can refute the suggestion that the purpose which figures to our fancy as a cause is in reality a mere epi-phenomenon, an attendant aspect of a causal process which is at bottom always mechanical.

12. For the behaviour of the living organism there are, in fact, three possible explanations. The first conceives it as a mechanism adjusted by a supernatural intelligence to respond to its environment in accordance with its needs. This endowment is to explain all the lower forms of animal behaviour, all that we have hinted at under the phrase 'organic adaptability,' together with reflex actions and probably 'instinct.' In addition, the same higher intelligence has endowed the human animal with a soul, and the higher brutes with a certain undefined measure of intelligence, to which their more distinctively purposive actions may be referred. Towards this soul or this intelligence the bodily instrument stands in the relation of a mechanism. It is not part of the mind, nor the mind part of it, but the two act and react. So far there is a clear-cut distinction, not so much between the teleological and the mechanical as between mind and matter. Matter never serves a purpose except when wrought into a systematic arrangement by a mind external to it, be the 
mind finite or infinite. So far the first view, from which we note that all that is not clearly purposive is clearly mechanical. The second view agrees that the basis of animal behaviour is a mechanism, arranged with greater or less plasticity to respond to the environment in the manner best adapted on the whole to secure the life of the individual, or, more properly, the permanence of the species. But it holds that this arrangement is not truly teleological. It has not been constructed by a supernatural mind, but has grown up through the remarkable combination in the substance or substances known as protoplasm, of the quality of modifiability with that of permanence. In virtue of this quality, protoplasmic tissue, which is strictly continuous from the first germ of life to its latest descendant, is for ever adapting itself in new ways to escape danger and surmount obstacles, and by an indirect but effective process, the steps of which need not be recapitulated here, there grows up a structure, which no mind planned to fit its environment, which no mind shaped to secure its ends, yet which does fit its environment, and thereby does secure its ends. Here again then we have a mechanical explanation of at least the lower form of vital activity, and the only doubt is how far the explanation is to extend. If reflex action and instinct, which already show evidences of plasticity are to be referred to an inherited arrangement of interacting parts, may it not be possible to gather the seemingly intelligent actions into the mechanical fold, and if so, will there be any fathomless gulf between the behaviour of animals and men? May not teleology itself, lately referred to mind, be simply the appearance presented by a mechanism too complex in its adjustment to details to be grasped in the entirety of its principles? Should not choice and effort and deliberation and, indeed, consciousness itself be set down as epi-phenomena which, in the inscrutable movement of things, have been evolved, interesting but devoid of function, as the accompaniments of those interactions of nerve-elements which, if we could understand them adequately, we should see to be governed in reality by purely mechanical laws?

To these questions the third theory offers the following 
reply. Whatever the cause or origin of the organism, it is in itself not a purely mechanical arrangement of parts. It is neither a machine created by intelligence $a b$ extra, nor one built up by unintelligent processes. It is not a pure machine at all, but a whole in which an organic, and that is a teleological, principle is at work within, operating on and modifying what are otherwise physical, mechanically determined elements, and so fashioning the growth and function of the parts by reference to the requirements of the whole.

Is there a possible logical proof of this theory? Can we, first, establish it for those organic actions which are accompanied in our consciousness by clear purpose? Can we justly say that the purpose causes the action? The reply is that our analysis of purpose has justified the application of the inductive test that has been briefly referred to. It shows that the question whether an act is purposive must be answered affirmatively if it is proved to depend on its tendency to yield certain results. Now comparative observation, both of our own purposes and of many actions of other human beings and even of animals, shows that in many cases action varies in accordance with this tendency and in relation to no other observable existent condition. Such action, therefore, must be purposive, unless there be some condition present in each case which we cannot observe, and this condition must (to exclude the alternative of teleology) be a collocation of forces acting mechanically. But a mechanism which can vary indefinitely in accordance with unique conditions differs radically from any mechanism that we know, the condition of a mechanism being that it responds in a typical way to typical conditions. A machine may achieve unique adaptations by a general arrangement so planned as to answer each different case that comes under some general rule in a different way. Thus in the linotype the spaces between the words are made by wedges, which are driven home by a single thrust, and owing to their shape go just far enough to fill the line. No two consecutive lines will, unless by rare accident, require precisely the same spacing, but the plan of thrusting in wedges secures the true fitting differing in each case yet equally adapted tc the end. The combination, however, though differing 
from case to case, is the same in principle. It is quite another matter when the principle of combination differs from one instance to another. In a simple purposive action, such as that which we first took as an illustration of purpose, where I require a book which I remember to have left in a particular place and go to fetch it, my memory, which, mechanically interpreted, must be some deposit of the effect of my previous dealing with the book in my brain, is so combined with my need and my physical surroundings as to discharge in succession the actions appropriate to fetching the book. This deposit-complex enough in that it must have its exact point to point correspondences with the several physical relations of the rooms of the house, etc.-is only one among the millions of deposits that my experience has formed. Yet provision must be made for selecting it out of them, and bringing it, and none other, to bear upon the physical tension, which may be supposed to correspond to my felt need, and thereby to effect the successive discharge of a complex series of actions. If we try to formulate a general plan for effecting such selection and correlation, we find ourselves speaking of a state of want, picking out from experience whatever is relevant to its satisfaction, and guiding action accordingly. But though we might find terms other than these which would avoid all reference to feeling or consciousness, the explanation would imply that there exists a something determined in its actions by their relation to their results, i.e. something purposive. Abstract the notion of the relevancy of means to end, and the bottom of the whole proceeding tumbles out. In short, in the activity which we claim as purposive, we find repeatedly that one factor of our life (e.g. an experience) may be brought to bear upon another (e.g. a want) in a manner that varies indefinitely from case to case. The only principle uniting the otherwise unique combinations is that of the relevance of the combination to the end. Admit this principle, and we recognise a structure determined by purpose. Deny it, and we have no general plan to explain the unique combinations. Either horn of the dilemma excludes mechanism. 
The denial of purposive causation, therefore, is not suggested but repelled by general experience, and owes its existence only to the theory that everything must act by mechanical laws. But this theory is a pure assumption, which derives its apparent cogency from confusion with the quite different principle that everything must act in accordance with some law. The leading mechanical principles I take to be adequately proved for mechanism, and, therefore, for any structure which is purely mechanical. Now the organism is a physical structure, but to assume that all its actions conform to mechanical laws is to assume that it is a physical structure only. Consciousness directly informs us that it is more than this-that it is what we called in Part I. Chapter II. a psycho-physical whole. How far the psychical elments in it, which our account has led us to conceive as activities constantly correlating the actions of its different parts, actually causes the reaction of these parts to diverge from the line that they would follow in accordance with purely mechanical laws, is a question which is to be settled entirely without prejudice by inductive argument. This argument shows, in fact, that psycho-physical wholes differ in their behaviour from purely physical systems in direct proportion to the development of the psychical element within them. As against the obvious inference from this argument, the mechanical view can only maintain the bare possibility that there might be a mechanism so constructed as to yield all the varying adaptations of the living being. This is a consideration to which, in view of the radically different character of known machines, very little weight would attach, but for the difficulty of the supposed breach of continuity involved in purposive action. But there is no breach of continuity. Purposive activity, i.e. the conditioning of the action of each part of a system by the causal tendency of the configuration as a whole, is the characteristic mode of reaction of certain structures-those which we call psycho-physical. Qua physical this structure tends to act in accordance with mechanical laws, but this action is modified by the condition mentioned, which is the psychical element of the whole in operation. If a body impinges on an arrangement of 
objects in a field of magnetic force, there will be a rearrangement of those objects in which the direct effects of the impact will be compounded with the governing conditions of the magnetic tension. When one element of the nervous system is affected by an external force, there will be a redistribution of the molecules within the system, regulated by the tensions of the system. Only these tensions are of a peculiar character. They bring to bear on the action of each element not only the existing condition of the whole, but its moving processes, what it has in it to become or bring about, its causal tendency. Such a tension is teleological, not mechanical, but it furthers, corrects or guides the motions of physical elements in the system to which it belongs, just as the magnetic or any other mechanical tension might do. Moreover, on its psychical as on its physical side the psycho-physical whole grows out of its antecedents just as any other configuration, only that in the action of its antecedents, the teleological condition will always have been operating. There is then no breach of continuity in teleological action, though there is involved the operation of conditions which are not those of a purely mechanical system.

I3. One further point remains. We have considered here only those cases of fully developed purpose, in which the idea of the end and of the tendency of the act towards the end is the condition of its operation. Our definition of purposive activity was drawn from these cases. But the definition would, in fact, include any act in which there is any element through which the causal tendency may operate. The question whether sensori-motor impulses or any lower kinds of organic activity are purposive then depends on the question whether they contain any such controlling conditions. Inductive methods suggest that they must do so, because such activities do vary in accordance with their tendencies to produce certain suitable results while maintaining themselves under varying conditions in the form which yields those results. From this we infer that the tendency to the result is a condition of the act, though we may not be able to identify the element 
through which this tendency works. In the case of sensori-motor action, however, I think we can identify the element which corresponds to the idea of a more developed purpose. As the organism moves towards an object, e.g. its prey, it is guided, i.e. its motions and efforts are determined, by the sense-perception of their relation to the object. But if we carefully exclude every ideal element from the sense-perception, we must banish with it every reference to what is about to come, or likely to come, out of the effort of the movement. We are thus left with the change which the effort is at any given time effecting, and the gratification or displeasure which it yields. We may readily conceive this attendant feeling to be a condition of the continuance of the effort in any given direction or of a modification thereof. In that case sensori-motor action will be controlled from moment to moment through its present tendency, e.g. the sense of approach to the prey will be directly gratifying. The suitability of the feeling, i.e. the adjustment of this passing gratification to final success in the effort, must be established a priori by those circuitous methods which we examined in Chapter III. The sensorimotor act, however, would come under our broad definition of purposive, as it would still be its tendency, though its tendency as measured in consciousness in the changes it is at the moment effecting, that conditions its action. We may, I think, carry this conception down to the lowest levels of conscious life, as we may suppose, e.g. that any movement giving relief from discomfort is thus sustained by the dim feeling of what it is doing. Finally, it is at any rate the simplest hypothesis to suppose that all true organic actions-as distinct from the reactions of a perfected living mechanism - are guided by some psychic element of this kind, and that it is through this psychic element that the residual state of the organism reacts on the behaviour of any given part. If that is so, every element of living tissue capable of genuine organic selfadaptation is affected, along with its mechanical interactions, by a psychic factor operating, whether with the rudimentary or fully developed character of a purpose. $\mathrm{Be}$ this as it may, the question whether any particular cause 
is purposive or mechanical becomes under our definition a question to be settled without prejudice by induction. If the multiform variations of process in a self-adapting living being can be explained as the result of a complex co-ordination of elements, let them be so explained. But if they cannot be so explained, let it not be assumed that the explanation must be sought on those lines or be for ever unattainable. ${ }^{1}$

${ }^{1}$ In this account the living being is regarded as a system of what must be called forces, in which mechanical relations are qualified by teleological relations. When these two sets of relations are hypostatised as Mind and Body they become two substances, and in place of a system whose mode of action as a whole departs from that of mechanical systems in virtue of its specific quality, we have the problem of interaction between two distinct and separate systems, each with laws of its own. If interaction is admitted, we have the conception of body as a purely mechanical system, whose operations at a certain point come plumply to an end, while at another point they as plumply begin, the intervening stage being filled by actions within the other system. Body is then a purely mechanical system which does not conform to laws which, it is not denied, are adequately proved for mechanical systems. To escape this conclusion it must be admitted that Mind exerts force and is acted on by force. But Mind was precisely the concentrated essence of that which is opposed to force. Thus the contradiction of a purely mechanical system which does not act mechanically is balanced by the contradiction of a non-mechanical system which does act mechanically. To escape from this dilemma the Parallelistic scheme is propounded, according to which the mental and the bodily run on side by side in point to point correspondence, but without interaction. This scheme, however, in effect renders the mental element superfluous. A complication of mechanism is all that is required to explain the actions of living beings. On the other hand, the rise of the psychical stream in coincidence with a certain point of the physical, and its disappearance at another point, are left unexplained.

In point of fact, the actions of living beings are not explicable in mechanical terms, and we are compelled by the evidence to admit a teleological factor. This we are able to do without contradiction if we avoid hypostatising qualifying aspects or conditions of real process into. substances. The concept of the mechanical sums up or brings together certain elements of experience; the concept of Mind certain other elements. But these elements belong to or qualify realities which act as. wholes, and may include many more elements which elude not only our observation but any inferences which we can draw from observation. The mechanical and the teleological are then modes in which reality operates. At some points reality appears to operate wholly on mechanical lines. At other points, in living beings, its mechanical operations are qualified by teleological factors. At other points, it may be, it acts. 


\section{DEVELOPMENT AND PURPOSE CHAP.IV}

in teleological ways exclusively. To avoid misunderstanding it should be added that there is no warrant in this statement for the inference that in the living being either the mechanical or the teleological factor is the 'substance' of which the other is the 'quality.' Both factors qualify the total Reality, which in addition may contain many unknown elements. 


\section{CHAPTER V}

\section{EVOLUTION AND TELEOLOGY}

r. If our analysis is just, the causal process may be reduced to one or other of two fundamentally distinct types. On the one hand we have the mechanical sequence conceived as a continuous flow in which each phase proceeds uniformly out of the preceding, unaffected either by any concomitant changes in the whole of nature or by the result to which it contributes. On the other hand, we have the organic or teleological-for we have seen that these two conceptions come down to one-in which the process is itself conditioned by its tendency to produce a given result, and in which consequently the elements in any complex whole are mutually determined by their interactions, out of which the resulting phase of the organism is to issue. We have now to enquire into the part played by these two processes in Reality as a whole. In this enquiry we obtain some light from specific experience, which undoubtedly shows us mechanical processes everywhere at work, but also, as we have seen reason to think, shows us teleological processes, which in the course of terrestrial evolution develop and expand their scope. Our synthesis, however, being too narrow to enable us to judge of the relative importance of the two elements in Reality as a whole, we have to look for some other instrument of enquiry.

But apart from the interrogation of experience, what instrument have we? If the term interrogation be taken in the widest sense, we may safely reply none at all. But for this purpose interrogation must be taken to include not only the results which experience demonstrates, but any 
truths implied in the process of demonstration. What on the surface we call a sound generalisation from experience is not, of course, a truth which merely sums up in a single formula a set of observed facts. It states a connection as holding generally, and if it is thoroughly scientific, universally; that is to say, as holding not only for certain things that we have observed, but for others that we may observe or that may never fall within the sphere of our own experience at all. Hence the scientific use of experience is a process which goes beyond observed and recorded facts, and makes a certain use of these facts for purposes of inference. Suppose that we can analyse this usage, and write down certain propositions which, if true, justify it, and, if false, destroy it. These propositions must then be regarded as the tacit assumption of the scientific use of experience. If they are true, the results of science are trustworthy, and if not, not. That being so, it is clear that whatever validity attaches to the results of science, whatever confidence we can legitimately place in its generalisations, must attach equally to these assumptions. Now it is open to thinkers to question whether science itself is valid, and if the answer be in the negative, this argument for the validity of its assumptions falls with the structure of science itself. I do not propose here to add anything to that which has been already said on the ultimate ground of rational thought, but assuming for present purposes the general validity of the scientific method of enquiry, I propose to consider some of the assumptions of science, and to see what light they throw on the problem before us.

2. As has been shown above, the impulse of rational thought stated generally is to weave its experiences into a systematic whole. The isolated unconnected experience is as it stands non-rational. It requires to be connected, to be shown as dependent upon some other element in experience, and as itself in turn a factor in the determination of other elements. That is to say, the work of thought, when summed up, is to build up a Whole of experience, in which each part is a condition and is also conditioned. Every element of the whole would have a reason rendered for it 
in something else, and ultimately in the nature of the whole as a whole. Reason may indeed be defined as the impulse to take things as parts of a whole (cf. p. 276).

Now we have seen above that the attempt to conceive Reality as a whole encounters all the difficulties derived from the idea of the Infinite. But let us, for the sake of argument, suppose it possible to overcome these difficulties, and to represent Reality as an intelligible whole. What we have to observe is that, in such a whole, for every part the reason given may be something that lies wholly or partly outside of it. One element is explicable by its relation to another, but of the Whole as a whole no such explanation can be given. Assuming that reason could achieve its ideal and form things into a whole, it could give no further account of the whole that it has formed. At first sight this alone appears as self-contradiction or selfdefeat in the work of Reason. Everything needs a reason to account for it, but of the whole of things no rational account can be given. But this is going too fast. It may be replied that as ex vi termini a whole differs from a part, the rational account of a whole is something different from the rational account of a part. The only reason that can be sought for the whole of things must be an inherent reason. The part may, and in a measure must, have its reason outside itself. The whole of things cannot have anything outside of it. Its reason is something in its own nature.

Now the only inherent reason for a thing of which we have any knowledge is its value. Mechanical causation as such points us beyond the effect to be explained. The value of a thing is inherent in the thing, and if we ask for any other explanation of the existence of valuable things it is not because they are valuable, but because value is not produced unconditionally. The whole then, we infer on these lines, has a reason for its existence in its value, but the value, the good of the whole, is in turn conditioned, just as something valuable to us is conditioned. It is conditioned by the structure of Reality, without which it could not be achieved. But here arises a formidable difficulty. If everything real is implicated in the structure 
of the whole, and if the structure is determined by its value, then equally every part exists for the contribution that it makes to the value of the whole, and Reality is Perfect. But if this is so, perfection loses all meaning, and the value which we attribute to the whole of things is so discrepant from what we recognise as value that all use of the term becomes misleading. It has brought us only to the edge of the gulf where the piety of optimism disappears into a whirl of unmeaning words. A retreat lies open, however, if we reconsider our conditions. Our postulate is a system in which every element is conditioned by every other. The structure of this system may be conditioned by its value, but the value is then an element within the system, a living principle running through the whole, but not identical with the whole. What we have done in effect is to look for the explanation of the whole, not to itself, which would be tautology, but to something within it ; not a part strictly, for the conception of division does not apply, but a determining character or condition. But the moment that this condition is clearly recognised as something less than the whole, it in turn requires to be conditioned, and we may take all the elements that go to make up reality as among its conditions. The relation then is mutual. Though the structure of reality exists because it has value, every element that goes to build it up is on its side, so far as it goes, a condition of the structure, and, therefore, of the value secured by it. The contribution of each element is certainly a condition of its existence in the structure of things, but its own inherent nature is in turn a condition of the structure, and, therefore, of the value which that structure possesses. Dualism remains in this account, but not, it may be urged, a dualism inconsistent with the nature of the system propounded. It was essential to this system that every part should be at once condition and conditioned, and now we find that, in fact, while everything that is real is fixed in its place by the structure of the whole, and is, therefore, dependent ultimately on the value which this structure secures, it is at the same time a condition, contributing on its part to determine the character of the whole which it helps to build up. The good 
then we may conceive to be dependent on the interaction of an infinity of conditions, and limited accordingly.

3. If we go on to ask what kind of value can be supposed to attach to reality as a whole, and of what nature are the conditions which at once determine and limit it, our partial experience does not leave us wholly in the lurch. We have spoken of the value of a thing as the one type that we know of an inherent reason. But within our experience such a reason never operates, as we have also remarked, unconditionally. It implies a mind animated by a purpose, operating on surrounding conditions so as to secure the thing of value. To the surrounding conditions, we ought to add those which go to make up and determine the nature of the purposing mind itself, to give it its bent and tendency. Under these conditions we get a process which is, in a measure, self-determining. In proportion as the purpose dominates it, that is to say, every element concerned is brought into being for the sake of what it effects, and the casual tendency of the process as a whole is itself the cause of the process. The purpose animating the mind is, as it were, the germ out of which the whole organised system of action grows, and it grows by bringing within its tissue, conditions necessary to its end, but it is also at every point limited by the degree in which these conditions are malleable, and the final character of its end must even accommodate itself to these. The conditions determine the end every whit as much as they are determined by it, and at bottom this is true, not only of the instruments with which the purposive intelligence acts, but of the conditions which determine the character and activity of the mind itself, which indeed are responsible for the initial fact, that this or that specific purpose is formed. Furthermore in the purposes of experience there are always conditions external to the purposive system and indifferent to it until shaped and arranged by the mind. Thus the purposive process is never wholly self-determining.

Now, if we seek to apply this model to Reality as a whole, the first steps are sufficiently clear. We have to conceive a mind operating on conditions under the inspiration of an 
idea and shaping the course of things to the final realisation of that idea. The difficulty arises when we contemplate the relation of the mind to the conditions operated upon. Our model suggests that in the beginning of things the Spirit moves upon the face of the waters of chaos, evolving out of them an ordered world. But this, once again, is sheer dualism, and our whole conception was founded on the necessity of conceiving an ultimate unity of the organic kind. If this is to be achieved, we must clearly bring the 'conditions' into closer relation to the purposive scheme. On one side, this is not difficult. We may, without inconsistency, suppose that everything real is so far conditioned by the Purposive principle that at one stage or another in the process it has an essential part to play. We might conceive, for instance, that its value in the scheme is not in its initial existence but in the use to which it may ultimately be put. If so, the real limiting condition of the Good would be the necessity of organisation. The elements of the Good would be here all along, but not in the form in which their value can be realised. The condition under which the Good can be reached would be the process of shaping, of development, by which harmony, in the full sense, is to be attained. We come then to the conception of the Good of the world as consisting in a Harmony achieved through development, a conception which accords well with the results of ethical analysis. But even so, dualism is not extirpated, for the operating mind is still set over against the conditions upon which it operates, and, however difficult it may be to imagine the relation, it is clear that if reality is an organic whole, this isolation must give place to' a conception of the mind as itself dependent on the conditions which yet it has to subdue. The strife which makes up the world-process may then be conceived more on the analogy of the efforts of the mind in man to subdue the body on which it depends for its own existence, or to the regenerative efforts of a self-governing society in relation to its own members, and the development implied in the idea of a gradually realised purpose, would be not merely a step by step reduction to order of a soulless material by an intelligence operating from without, but 
equally and more truly a development of mind itself. That is to say, it would be on a vaster scale a process analogous to that which we have traced in the successive stages of human evolution.

4. As thus stated, the argument assumes that we can, and therefore that we should, form for ourselves an intelligible account of Reality as a whole. It is, we have seen, possible to question this assumption and to contend that the utmost that our thought can postulate is a system completely intelligible in itself, but not exhaustive of reality. Such a demand, it may be said, meets the needs and corresponds more nearly to the actual position of our cognitive powers. For it may be said, we may suppose a fabric of knowledge so compact that it stands as a single truth, and so fully articulated that the whole meaning of every fragment is clearly visible in the bearing of every bit of it upon the rest. Such a system would possess the maximum of certain and rational explication. Yet, taken as a whole, it must be said to be a mere datum. Why does it exist? Why should anything exist? Would its internal completeness necessarily bar these questions? On the contrary, if we face them fairly, may they not deliver us from the dilemma of the infinite process? Let us consider the position. We assume as before, that the rational impulse is to weave experience into a connected whole. But we do not postulate that this whole must be co-extensive with reality. We postulate only that it should be intrinsically intelligible and should include all that comes or can come within our experience. Of such a whole, it is still possible to ask how or why it came into being, but it is not possible to ask this question of any part as opposed to the whole. Every part is fully explained within the whole, and this will apply to the whole itself at any given moment of its existence, which is, of course, a part as compared to the entire stretch of time through which it persists.

Now the essence of such a system has been seen to be that there should be contained within it nothing unconditioned. If, then, we seek to trace back the genesis of things within it on mechanical principles to an initial cause, we are 
at once confronted by two alternatives. Either we must take this cause - a mere part of our system - as an unconditioned datum, or we must go beyond the system to an anterior existence. In either case, the system fails to realise its pretensions. It follows, that in a complete system, any point that we start from as the cause of what follows, must itself be determined by what follows, which is possible only if its inherent tendency to bring about the resulting process is the condition of its own existence. That is to say, it must be purposive, and we are brought back to the same conception of the process of reality, as conditioning and conditioned by purpose, as before. But the result we have now reached is this-that whatever be our capacity or incapacity to deal with Reality as a whole, whatever within Reality is fully understood, is part of an organic system. If this is so, this organicity is true of Reality as a whole, not in the sense that it is only in a summed-up infinite that it is realised, but in the sense that it expresses the final central essence of the Real, wherever it be taken, provided it be fully understood.

The organic character of Reality then is deducible from a postulate of thought. But what validity attaches to such a postulate? If it is something so implied in the reasoning process that, if it is true, reasoning is valid, and if it is false, reasoning is invalid, then its certainty is equal to that of any rationally established truth, and with that result, after our previous enquiry into the validity of reasoning, we are content.

But can this be shown? What is postulated is the possibility of a completed system. Such a system is the ideal of thought, but can we say that its possibility is a principle without which thought cannot work? It is one thing to say that the work of thought is that of systematising, and another to postulate that its work will some day be complete. Provided the principles that it uses in the work of systematising be sound, is it not possible that the work should go on without end, the value lying always in the solid fabric achieved at any given time and in the living impulse to extend it, not in the goal or ideal towards which the impulse appears to move? Perhaps the fable of the 
treasure digger applies. The gold was not there, but the digging itself produced golden value in the soil. There are features in the history of discovery which suggest an analogous truth in this relation. By patient work we build, but not as we planned. If this is true of the search for the philosopher's stone, may it not be as true of the search for any completeness of understanding?

Before yielding to this doubt, we shall do well to look more closely at the actual course of thought. We have spoken of the solid fabric already reared as having intrinsic value, but when we look at the actual structure of knowledge, even of the physical science, nay, even under certain aspects of Mathematics itself, this solidity is not so easily to be seen. Everywhere, as we approach the wider and deeper conceptions-conceptions which make up the very tissue of our experience, such conceptions as Space, Time, Number, Matter, Force, Energy, Life, Thought, Consciousness, Morality - we enter a region, not of rocklike stability, but of a fluidity of which the best that we can hope is that it is the fluidity of growth. The advance of experience does not merely add grain after grain to a heap that is accumulated once for all. There is addition, but with addition there is also constant modification, and few, if any, are the truths of which we can say with confidence that they can never be modified. Perhaps there are none even in Arithmetic of which the total interpretation may be regarded as finally and irrevocably fixed. The advance of knowledge is a process of modifying conceptions. But if this is so, what validity, it may well be asked, attaches to the conceptions already formed, and to the thought which engenders them? If rational methods do not yield us truth, what do they yield us whereby we may put confidence in them? The answer is hard to find unless we remember that modification is necessary to growth, and conceive reason, accordingly, as an impulse towards harmony which, however incomplete at any given time, is always moving in the right direction. If this conception be admitted, it becomes intelligible that a method should be valid though its immediate result does not possess final truth. The validity of the method rests in this, that it is 
essential to the movement towards truth, and the test is that by constantly following such methods we arrive repeatedly at a wider and more thorough harmony, we reach points of view which do not simply negate old ones, but rather absorb them and set them in their place as partial and incomplete elements. But so to conceive knowledge is to conceive it as essentially a growth. The time will come when sacred Ilium, the highest point of view which we can reach or the inmost citadel of our faith in life, will in turn be overcome. Within the Empire of a greater truth it will figure as a detail which we misunderstood while we cherished it. Thus, if our confidence in anything that we can know or believe has reasonable justification, it is not because that thing is known once for all, but because it is a genuine and essential phase in the growing formation of truth.

But if, in the search for logic and for certainty, we are thrown back on growth, the lines of growth assume a fundamental importance. Whatever we know of them becomes the most vital part of our knowledge, and though here, again, we are fully subject to all the old limitations in forming our point of view, yet it remains that the best conception attainable of the movement is necessary to the full formulation of the reasonable and the true as far as we can know them. If the life of rational thought consists in development on certain lines, to say that development can never mature is to threaten the life itself.

But may it not be, the doubting question will recur, that in formulating the conditions of development we are tempted to ask too much. Let us admit that Reason is at bottom the impulse towards the comprehension of Reality as an organic system. Is it not possible that this impulse, valid and valuable within its own limits, is yet applicable only to part of Reality? May there not be an irrational element, essentially inexplicable, irreducible to system, knowable as we know brute facts that are not explained, not intelligible because not in fact conforming to the conditions of intelligibility? Let us observe, first, that if this be true it would not affect one part of our previous reasoning. Reality, as far as it is intelligible, would fall within 
one system, and from this it would be deducible that it would form an organic whole, with a development determined by purpose and moving towards more perfect harmony of organisation. Such a system, it may be said, is real, and to discover and understand it is the goal of our rational endeavour. On the practical side, to make a corresponding whole is the endeavour of the rational in action. But though contained within the real it is not all that is real. In part, the real is fundamentally irrational, and that is the source of the ubiquitous limiting conditions which give us so much trouble when we seek to absorb them into our system. Dualism would then be a final truth, yet not such a dualism as to impair the validity of the effort of our minds, both practical and speculative, towards unity of system.

5. Between these possibilities, I doubt if it would be possible to decide on the ground hitherto taken. As long as we are depending on an ideal of thought, we are relying on a conception which must have value if the effort of thought has value, but which need not necessarily be true. Let us then revert from the ideals of thought to the principles actually implied in the process of reasoning. One of these principles, as previously shown, is the Law of Universal Causation. If that is so, then, according to our present argument, the general statement 'Every event in time has a cause,' or 'Every event in time has an antecedent such that, from precisely similar antecedents, precisely similar events always follow, is a generalisation which must be true of Reality as a whole, if the methods of science are sound. That is to say, from the general validity of reasoning, we can infer, as being implied therein, a general truth about reality. The truth in question is one which concerns causation, and if we can thus deal with causation in general, it is possible that we can deal in a similar way and no less cogently with the two species of causation which we have distinguished. That at least is the experiment which we are about to try.

In the first place, the precise assumption which we make, when we enquire into the interconnection of distinct 
elements in our experience, is one that may be formulated in several ways. But however formulated, it comes down to this-that the attachments of any element, its relations, that is to say, to other elements, be these relations in space, or in time, or relations of any other kind, are dependent on the character of the elements considered. Thus, if we take any two elements as $A$ and $B$ in a given relation, we have on our assumption two alternatives. This relation may be dependent on the character of A and B as such, and on nothing else. If this is so in the fullest sense, it follows that the relation must be mutual or convertible. $A$ is such that if we trace out the constituents of its character in full, we find ourselves led to the assertion or recognition of $\mathrm{B}$ in the relation to $\mathrm{A}$ originally postulated, and the converse result happens if we start from B. B as such involves $A$. The simplest illustrations of such a relation are to be seen in certain cases of continuity and in the more elementary relations of time, space and quantitythe constructive relations, as they may be generically called. The simplest case of all is one in which the two terms in ordinary speech coalesce into one. It is that of the persistent identity which is the basis of our idea of substance. Suppose our term A to be something existing in time and dependent for its existence on no conditions outside itself. Then its own nature $\mathrm{A}$ which conserves it in time for as short a duration as we choose to take, conserves it indefinitely. The second term of the relation B is simply the existence of the same thing at any subsequent point of time, and this is necessitated by $\mathrm{A}$ alone, while $\mathrm{A}$ - a pre-existing phase-is similarly inferable from $B$. The one term is the other produced forwards or backwards in time. It may be said that we never in experience actually achieve knowledge of substance-that is, of such self-contained existence. There are always conditions, $\mathrm{X}$, many of them unknown, on which the persistence of $A$ in reality depends. But this is not for the moment the point in question. We have simply to illustrate the meaning of an 'as such relation.' We may easily be mistaken in taking for them relations which are in fact contingent. But the mistake consists in attributing a self-contained existence to 
A instead of to AX. AX (even if $\mathrm{X}$ prove ultimately to be the entire system of reality) will serve in its successive phases as a type of an 'as such relation.' With the same limitations, it will be understood that the successive phases of a uniform process of motion in a straight line or upon a uniform curve, may be held to imply one another as such. The motion may depend on conditions outside the moving body, but wherever these conditions are given so that the first phase comes into being, the remainder is intelligible from that phase alone and needs no further assumption to explain it. Again, more complex changes or processes are derivable from their antecedents ' as such,' when they are referable to the impact of elementary processes upon one another, and when the characteristic of each elementary process is still traceable in the result. Thus, in the typical case of mechanical impact, we have two masses $M$ and $m$ moving with velocities $V$ and $v$. If unaffected by one another or by any third body they would continue to move in straight lines. But from the paths they are describing, we may be able to see that 'as such' they determine the collision that ensues, and in this collision, if the bodies are elastic, there is a change of motion which is to be understood if we take either body, say $M$ with its momentum which is $\mathrm{MV}$, and conceive this altered in quantity and direction by the amount $m v$. This gives us the new momentum $\mathrm{MV}^{\prime}$ where the effect of $m v$ is preserved as a modification, in quantity and direction, of MV. Similarly, MV survives in the modification $m v^{\prime}$ effected in $m v$, the proof being that the whole resultant momentum $\mathrm{MV}^{\prime}+m v^{\prime}=$ the original momentum $\mathrm{MV}+m v$. If the bodies are not elastic, some of the momentum is lost, but a proportionate amount of heat or other energy is evolved - proportionate, because by appropriate means it is again convertible into motion equivalent to the missing quantity. The object of mechanics is to get down to equations of this type in which the sum of the elements of a process is seen to remain constant before and after a critical change, an equation which indicates that each element in the new condition of things is the equivalent of a former element as modified by its new concomitants. Mechanics is thus 
constantly resolving complex interchanges of energy into sequences and combinations in which each term is to be attributed to some antecedent 'as such.'

But as the infinite variety of experience shows, the actual relation that we find between any two of its elements is by no means necessarily uniform. It may vary to any degree, but the assumption of our scientific reasoning is that the variable relation is ultimately traceable to uniform relations, and that is to relations dependent on the intrinsic character of the terms 'as such.' 'The inductive part of the work of science consists in the endeavour to sort out relations, and determine which of them are due to extrinsic and variable conditions, and which are self-dependent, or hold between the terms ' as such.' Here, and particularly in relation to processes in time, the conception of continuity lies at hand as a key. We conceive what we find existing now as a phase in a continuous stream of being which issues in it as from this phase in turn another will issue. In some respects the stream of being is unchanging, and that which is unchanging we conceive as the substance underlying or, if we prefer it, constituting the permanent elements of the process. Whatever, in the course of critical changes, is found empirically to be permanent, is thus apt to be conceived as substance, i.e. as self-determining in unchanged character and therefore as permanent. Thus there was a strong tendency to attribute substantiality to the Daltonian atoms when it was seen that in chemical transformations the 'elements' were not destroyed but were simply combined, re-combined or disunited as the case may be. Similarly, the constancy of energy, empirically established to a very close approximation in diverse and very critical cases, suggests the assignment to energy of the substantial characteristics of reality, and leads many writers avowedly or tacitly to treat energy itself as equivalent to substance, and its conservation as an axiom equivalent to that of the indestructibility of substance.

6. Turning from the persistent to the changing, we find two distinct problems. On the one hand, there may be a succession of changing states following in sequence, as 
A-B-C, etc. Such a sequence, though involving changes, may be uniform in the sense that given $A$, its change into $B$ and $\mathrm{C}$ regularly follows, and it may be possible to show inductively that this sequence depends on no external conditions. If so, A 'as such' yields B and C. Further, we may be able to 'explain' this sequence if we can analyse A into elements which, either severally or in their action upon another, can be viewed as a process of transition towards B. It is, in fact, only when we can thus regard change as continuous that we can be said to understand it. Explanation of events in series, then, resolves itself into the search for continuity in permanence or continuity in change.

But, secondly, the problem of change may be of quite another kind. Comparing two cases that are partly similar - two cases, say, of A-we find them to be alike in one respect and different in others-there, for instance, as $\mathrm{AB}$, here as $A D$. How are we to reduce variation of this kind to uniformity? We begin, as a rule, with a search for antecedents. $\mathrm{A}$ is explained as the effect of $\alpha, \mathrm{B}$ of $\beta$, and so on. But it is clear that we do not, on these lines, resolve the variable relation $A B$ into a uniform relation, i.e. one that holds between the terms as such. We may find an antecedent $\alpha$ which, as such, gives rise to $A$, and an antecedent $\beta$ which, as such, gives rise to $B$. But it will be only the relation $\alpha \beta$ which gives rise to $A B$. Now if the relation $\alpha \beta$ holds between the terms as such, it is uniform, and it follows that the relation $A B$ is uniform also. But $A B$ varies, and it was its variation which we had to resolve into uniformity. It is clear that we cannot succeed on these lines. The relation $\alpha \beta$ must also be variable. If we ask, in turn, for the uniform relation which is to explain $i t$, and look for it in the antecedents of $\alpha$ and $\beta$ severally the same argument will repeat itself and so ad infinitum.

A variable collocation $\mathrm{AB}$, then, has antecedents $\alpha \beta$ or $a b$, which also form a variable collocation. We shall not, by tracing the antecedents further, find a relation holding between the terms as such. There must be a condition of the collocation outside the related terms as such, and outside the series of their causes as such. Let us call it C. Then the position must be that given $C$ we have $A B$, 
and given $A B$ we have $C$, but we may have $A$ or $B$ without C. We have, in fact, to assume a relation which we may write $(\mathrm{AB}) c$, and we may take the antecedents in time of this collocation to be $(a b) c$.

Now there are two possible interpretations of this relationship. The first turns on the Plurality of causes. The antecedent $(a b) c$ may be written as a single cause $\gamma$. It is the cause of $\mathrm{A}$ and $\mathrm{B}$ in this instance. But there are also other causes $\delta$ etc. of $\mathrm{A}$ (and perhaps of $\mathrm{B}$ as well). But $\delta$ yields $A D$, so that if we have $A B$ here and $A D$ there, it is because in one case the cause is $\gamma$, and in another $\delta$. Before considering this possibility, let us examine the alternative. It may be that $c$ is not a cause of $\mathrm{A}$ or $\mathrm{B}$, but a condition of the combination of their causes which are $a$ and $b$, while it has its own direct effect $C$. Thus the existence of the combined process leading from $a b$ to $\mathrm{AB}$ is conditioned by the process $c-C$. And unless we are to bring in a fourth term, the necessity must be reciprocal, so that if we are to find a whole in which variable collocations are reduced to relations which hold of the parts as such, it must be one in which a certain collocation of elements implies and is implied by the existence of another element in the whole, which element itself may or may not be a collocation of more elementary parts. We have then a reciprocal relation, a mutual necessity as between two simultaneous things or processes.

In this, which constitutes the simplest case of conditioned variation, we should have two processes necessary to one another at successive stages. It would be indifferent whether we said that $A B$ and $C$ were mutually necessary, or $a b$ and $c$. But this indifference depends on the assumption that $c$ has a single effect $C$. It is equally possible that $c$ may enter into other relations besides its relation to $a b$, and in these combinations produce effects D, E, varying from case to case. If that is so, we shall not find anything co-existent with $\mathrm{AB}$ which implies and is implied by it. We shall have to explain $A B$ by reference, either to an antecedent combination $(a b) c$, or teleologically to some subsequent combination $(\alpha \beta) \gamma$. All that we can say is that $\mathrm{AB}$ must either belong to a whole, of which the parts 
necessitate each other, or be determined by such a whole.

Let us now consider the former interpretation-by the supposed Plurality of causes. By this $(a b) c=\gamma$, and $\gamma$ is one cause of $A B$. We may, in fact, ignore $a b$. They need not be postulated, for A and B may have many causes, and in the given instance, we may write the cause $c$. In some other instance of $\mathrm{A}$, the cause may be $d$, and so we get variety. But if $c$ is the sole and sufficient cause of $A$ and $B$, and if $A$ and $B$ are two distinct things or processes-and this is the case to be explained-there are only two alternatives. Either $c$ is itself a combination of two elements $\alpha$ and $\beta$, which in their interaction are producing $A$ and $B$, or it must be itself a process of change giving rise to $A B$. For, if $c$ is distinct in character from both $A$ and $B$, and is not a process that is simultaneously directed towards both of them, the change which produces them cannot be due to a simple continuation of this process. It is therefore due to something acting on $c$, which is contrary to the supposition that $c$ is the sole and sufficient cause. But further, if $c$ is something which has no distinguishable elements and is not acted on from without, it may be a process of becoming, but whatever it is becoming, it is becoming as a unity, that is, it must be one thing, not two things. If, for example, it is something quite homogeneous throughout, it may be changing, but it must be changing homogeneously. If one part were becoming $A$ while another was becoming $B$, there would be a variation without a cause of difference. It follows that $c$ must consist of parts $\alpha$ and $\beta$, which, either independently or in their mutual interaction, are becoming $A$ and $B$. Thus the cause of a complex effect must itself be complex. The combination of process-factors which form it, again, must either be due to their intrinsic character or to something external. But in the latter case, once more, the factors are not the total cause of $A B$. It follows that the cause of $A B$ must be a set of factors whose combination is due to their intrinsic character.

The result is that a variable relation $A B$ must either be a part of a whole $(\mathrm{AB}) \mathrm{C}$, the elements of which necessitate 
one another, or it must be referable to a cause, the elements of which necessitate one another. Now let us consider such a whole. Let us call it $\mathrm{M}$ and enquire into its relations with any other concomitants as $\mathrm{L}$ or $\mathrm{N}$. There are two alternatives, either it is related to $\mathrm{N}$, say, as such, or the collocation $\mathrm{MN}$ is variable. In the latter case, the same principles apply, and there must be a whole $\mathrm{P}$ in which $\mathrm{M}$ and $\mathrm{N}$ are reciprocally necessary, or in which $\mu, \nu$, determining the relation $\mathrm{MN}$, are so. This argument must be repeated with regard to anything standing in relation to $M$. If variable relations are to be resolved throughout into 'as such' relations, each variable collocation must have a place determined by and determining other relations, and ultimately related, in this reciprocal manner, to an entire system.

7. This mutual determination of parts must therefore run through Reality as a whole, and we are led accordingly to conceive of Reality, either as being at any given moment a system of parts which necessitate one another, or as being a collocation determined by such a system. Thus in the actual scheme of things existing at any given moment, any part has its determinate relation to the whole system. This relation, i.e. the existence and character of the part in its precise position, is determined, either by an antecedent collocation of reality out of which it grew, or by a future collocation into which it is to grow, or by the actual and existent nature of the part and the system as such. The two former cases, then, throw us backward or forward as the case may be. That is, if the relation of the moment is not determined by the nature of the terms as such, it depends on a relation which is so determined. But if any existing relation of a given part to the whole is something fixed by the character of the whole and part as such, if, that is, such a whole, as such, necessitates the existence of such a part in such a relation to it, and if, conversely, the part necessitates other parts, and through them, finally, the whole, that is a relation of mutual necessity, mutual support, or harmony, and if the same relation pervades all the parts, there is a harmony throughout them. They 
necessitate each other's existence, i.e. no one can exist for any time without the other. The totality of things at any time, then, either is a harmony or is a collocation derived from a harmony, either as proceeding out of one or as leading up to and existing for the sake of one. ${ }^{1}$

Now in a harmonious whole, any constituent part $a b$, with a constitution of its own dictating an orderly series of internal changes (or preserving itself unchanged, as the case may be), is maintained in this, which we may call its internal life, by the residue, such maintenance being a condition of the action of that residue. Such a whole then may be destroyed from without, but not from within. It follows, that if Reality as a whole is organic, its harmony is indestructible, and within it every part must pursue its own orderly life, contributing thereby to the life of the remainder. But since discord exists such a harmony does not exist and never has existed. It follows, that if its existence is implied in the structure of experience, that existence must be in the future, and the actual constitution of things at any time must be determined by the element in that harmony which each one of them is to contribute. ${ }^{2}$

${ }^{1}$ It may be said that this conclusion requires us to conceive of the universe as a definite sum of being, whereas being infinite it cannot be summed. This is not the case. We can make true assertions of infinite scope, e.g. that all pairs of two make four, though we cannot exhaust the numbers of pairs. The conclusion before us asserts that whatever exists is or is dependent on a harmony which must extend to all co-existent realities whatever and how many soever they be.

${ }^{2}$ It may be said that the conception of harmony only proves that the ultimate elements of the world order are indestructible, a fact already known to the mechanical theory. A, B, C imply one another, but they also interact and produce $a, \beta, \gamma$, which may involve a destruction of what from our human point of view is of value in the combination. But this argument ignores the essential point, that it is not the mere existence of elements which is involved in harmony but their particular collocation. A must be understood as a combination of elements $a \alpha, B$ as a combination $b \beta$, and the harmony involves the maintenance of the principle of combination. But in that case, again, it may be said we prove too much. Harmony must arrest all change, and this arrest is death. Not so, $a a$ may have its own internal process of change, and so may $b \beta$. The harmony between them means that the one series of changes is a necessary condition of the other. Hence harmony is compatible with change, but with only that kind of change which is the product of the internal development of each part. 
By this reasoning, while it is true that its contribution to an ultimate harmony is a condition of everything that exists, it is equally true that the harmony is conditioned by everything that exists. Harmony alone does not explain existence or the world would be already perfect. What exists must be capable of harmonisation, and the configuration which it assumes at any time must be one determined ultimately by the effort towards harmony. But it is an impulse which requires the whole evolutionary process to effect it. The elements within which it works, then, are originally by no means a harmony. As they stand they are indifferent, and in consequence the behaviour of any one may clash and collide with that of any other, and any partially formed order or combination of elements may be broken up by the action of others. This is the element of disharmony or discord which is not merely an empirical fact but a natural consequence of the mechanical principle which runs through the real order and was, in fact, the starting-point of the reasoning which led to the conception of harmony. The result of this reasoning is not to extrude mechanism, but to show that it must be conditioned by relation to an ultimate harmony, while harmony is equally conditioned by mechanism. The relation is mutual and the outcome is the process of development.

But discord or disharmony is not the same thing as disorder. There is at any time some definite configuration of the then existing elements, and we may conceive this configuration as at once determining the elements and determined by them. But so far as it is not determined by them, it is determined by its tendency towards a harmonious system. The principle running through the configuration then is not a static principle. It is an impulse towards harmony and effects itself by a development which transforms the conditions limiting or thwarting it, and renders them subservient to its ends. Further, an impulse to an end implies something of the nature of Mind, and we are thus led back to the same result which we reached by conceiving Reality as a whole conditioned by an element of value. We are led to conceive a mind limited by its own constitutive conditions and making its way by trans- 
forming or recasting them to the realisation of its full capacity. But for the present we may best state the relation of the different factors of the real in more abstract terms. According to our account, then, Reality would be at any given time a system of elements conditioning and conditioned by a principle of organisation leading up to an ultimate harmony, and through this principle everything real would be thus related to the harmony. In the more ultimate sense in which Reality is not in time, but time is in Reality, the whole system of things would consist in the realisation of an organic principle through development, time itself depending on the function of change as necessary to development. 


\section{CHAPTER VI}

\section{DEVELOPMENT AND HARMONY}

I. WE may obtain some fresh light on the movement towards harmony by starting anew from the conception of development, and asking in general terms what development is. What is meant when, comparing two individuals or two types, we say that one is more developed, more mature, more highly evolved; the other relatively crude, undeveloped, rudimentary? (I) We may use the expression with reference to some assignable character and its presumed genesis. Here, e.g. is a given organ of a given animal. It has such and such a structure and function, definite and clearly marked. By the aid of embryology we can trace it back through successive stages to a certain portion of a layer of undifferentiated cellular tissue. It begins, that is to say, by being something generic, to all appearance like other cellular tissue of the same individual, and not only of the same individual, but of embryos generally at that stage of their growth. From this it differentiates out, acquiring by a continuous process a character which is more and more distinct. The development of such an organ then has a perfectly clear meaning. It is a name for the continuous process of modification by which an object of distinct and well-marked character comes to attain that character. (2) Thus, when we speak of the development of something definite, there is no particular difficulty about the import of the term. It is when we speak of development in general and oppose it to arrest or decay that the question of meaning arises. When the animal dies and the organ that has been in 
question undergoes decomposition along with the body as a whole, a new process of continuous modification sets in by which a new result is arrived at, but we do not think of this process as one of development, but rather as one of decay. We might indeed, relatively to the idea of decomposition, still use the term development - the decomposition is more or less advanced, it progresses from small beginnings, becomes well marked and then complete. But without the qualification we should never use the term development of this process. It is just the reverse of development. Thus we seem clearly to have and to apply some notion of development in general, as a process having a certain distinctive character or trend opposed to a reverse process which we call that of dissolution or decay, and it is clearly this general sense that we shall require if we are to speak intelligibly of the world-process as a whole as a process of development. To begin with, in the instance taken, which is typical enough, we clearly predicate development of the process by which the organ acquires distinctive character, and this distinctiveness, again, involves a combined arrangement of parts, a certain formation involving a joint working of tissues-as, for example, the attachments of muscle, tendon and bone that make up the essential mechanism of a limb. There is in the ordinary phrase a combined advance in differentiation and integration, and this combination only becomes more conspicuous if we turn from the single organ or limb to the entire organism. It is, as compared with any stage of the embryo, highly differentiated, while it is also, as compared with any stage but the very first in which the embryo is a single cell, more completely integrated-its parts, that is, are more definitely adapted to the requirements of combined action. Putting the two points together, we find that what has happened is an extension of the organic character, and that is, again, a more complete co-operation of a greater aggregate of parts and of forces.

2. The paradox in the conception of development, and the standing difficulty in all theories of its nature and conditions, lies in the question in what sense the germ can be 
said to contain the developed organism. If the undifferentiated cells are really undifferentiated, if, that is to say, they are in their inmost structure just like any other cells, why do they give rise to this particular organ or organism and not to another? If, on the other hand, they are really different from the first, the process of differentiation is only apparent. The adult is preformed in the embryo, and what really happens would seem to be rather a sort of unveiling of what is already there, than a true growth of something new. How far does our slight account of development enable us to turn this difficulty? Let us take up the question again at a different point. Mechanics tells us that in any system, as long as it is uninfluenced from without, the sum of energy is constant. But any portion of this energy may be unapparent. For example, a weight lying on a scale is also suspended from a point by a string so that it barely presses the scale. In this condition it is in equilibrium. This means that several forces ${ }^{1}$ are being exerted, and in particular the downward pull of the weight and the upward pull of the string; but these forces are balanced. So far as any change, any movement is concerned, they are opposing forces and they cancel out. If the arrangement is disturbed by the snapping of the string, the balance is destroyed, the downward thrust of the weight has its effect. There is a transference of potential into kinetic energy, and there is something that may be called an evolution of motion and a performance of work. In fact, we speak often of an evolution or development of specific forms of energy as of sound, heat, light, an electric current and so forth. We may, indeed, conceive kinetic as a development of potential energy, and we observe that the mode of development is this-that where there is potential energy certain forces oppose and balance one another, and that given a readjustment which removes this opposition, each force has its own way and works itself out in some appropriate movement.

The kinetic energy liberated may theoretically perpetuate itself indefinitely, e.g. a projectile launched on a

${ }^{1}$ Meaning here and in the rest of the discussion by a 'force' whatever tends to produce or inhibit motion, or, generally, any change. 
path free from all interfering forces would maintain its motion eternally in a straight line, or if moving in a frictionless medium within the influence of some large body, would rotate about it as a planet. But, normally, the motion of one body is interfered with by others and it is brought to rest. Some of its kinetic energy is then re-translated into potential, but the whole is never restored. On the cessation of molar motion the kinetic energy takes other forms, and some portion of it is always frittered down into heat. If the heat could as a whole be collected again and brought to a focus, the sum of the original energy would be restored and the entire system in its new form would possess a potential energy equal to that with which it originally started. But this physicists believe to be impossible. They accordingly draw a distinction between energy as such, and the energy which can or could be made available to do mechanical work, and tell us that while the sum of the former is constant, that of the latter is perpetually diminishing. There is a steady dissipation of available energy measured by the increase of ' entropy.' Thus the mechanical view of the universe, in strange contrast with that of biology, psychology, and, as we may now add, of astronomy, chemistry and the physical theories of matter, contemplates a process of steady degradation or dissolution rather than a process of evolution or development. We start with a system of energy stored in many centres of high potential, and as we trace its liberation from these centres and the display of its nature in motion, we have to recognise at every point a final dissipation into a form in which it can no longer produce any recognisable effects.

That this can be a full account of the universal process is impossible, for the simple reason that it gives no account of the original storage. It assumes in Ostwald's way of putting the matter, a perpetual transference of energy from the points of greatest difference of potential to a state nearer to equilibrium, but it gives no account of the manner in which the difference of potential originally arose. It is clearly a one-sided account, as might be expected of a purely mechanical view, and rightly interpreted it is an 
admission of the inadequacy of mechanics to explain Reality. So much in passing, but let us note for our purpose that the reason why heat is imperfectly available for the purpose of restoring an equivalent of mechanical energy is understood to be the random and chaotic character of the molecular movements by which, physically speaking, it is constituted. If in the equilibrium which yields a store of potential energy, we have opposed forces cancelling one another and so yielding a zero of any apparent effect or real change, in heat we appear to have a chaos of movements producing no combined effect because not reinforcing one another in any given direction, but working against or across one another to no certain end. As soon as we can concentrate heat in sufficient amount upon, say, a certain mass of water, we get a definite series of distinct changes and the evolution of steam which can do mechanical work.

These mechanical considerations are of value to us mainly as yielding a hint of the true relation between the potential and the actual in development. We find that on the mechanical plane, (I) the energy operating in any process of change always pre-exists, but the forces possessing this energy have been inhibited by counter forces of equal energy until the process of change began; (2) that what the physicists call available energy, and what we may consider as energy in a relatively developed stage, is the energy of forces operating in some orderly way, that is, with some assignable direction or relation between the phases of change which they determine, while the same quantum of unavailable, or, as we may call it, relatively undeveloped energy is that of forces which operate in no concert with one another, not reinforcing one another, but in random cross currents that tend to no specific result. In effect, they tend to cancel one another as do the counteracting forces in stored energy. We then see that even at this stage, that which appears higher or more developed consists in a process in which we can recognise something that we call at least an order, and that is some arrangement of parts or phases of a process so as together to constitute a whole of distinct character. Such an order involves either the 
joint working of several forces or, at lowest, the liberation of one force from anything that counteracts it. Mechanically considered-and the whole process of Reality is mechanically conditioned-development consists in the rearrangement of forces so that from a state in which they conflict with one another and produce no regular series of changes (potential energy), they come to work in definite relation with one another, so that while each is responsible for some series of changes or some feature of such a series (kinetic energy), they together build up structures of definite types and relatively enduring character.

This process of development begins within the inanimate world, and that is why I have used the term structure instead of that of organism. Every organism is a structure, but not every structure is an organism. A structure is a whole constituted and maintained by interacting parts. The behaviour of each part is affected by that of others in some way which is distinctive and which is such as to give to the whole a definite character and a definite mode of behaviour-a line of action persisting in time which will only be modified by the infringement of some external force. The solar system is such a structure. It would appear that the chemical atom is such a structure, its elements being the corpuscles, and the binding force the electrical attractions and repulsions that constrain corpuscles to assume certain alternative mutual relations. Atoms brought within the sphere of mutual influence can modify one another, and form higher structures, which are molecules of the chemical compounds. But in all mechanical structures, and the chemical is assumed to rank ultimately with the mechanical, though the parts influence each other's behaviour, the action due to each is unaffected by the remainder. The mode of action characteristic of the part persists unchanged in whatever combination it may be found. Every force in a mechanical structure operates with its own magnitude and in its own direction, and, if the rest of the structure were suddenly dissolved, would continue to operate in precisely the same way. Only, as any element operating with such force is at the same time operated upon by other elements of the system, the actual 
behaviour of that element is a new product determined by the composition of its own forces with those acting upon it. In an organic structure, on the other hand, the union is more intimate. Though every element of the organism has its own character, this character stands as such in relation to the character of the whole to which the element belongs, and if that element is removed from the whole it is modified or destroyed. There is not only a specific interaction of parts, but an interdependence of parts complete in proportion as the organic character is developed. In the organisms that we know, and so far as we know them, this completeness is never fully realised. In the living organism the material particles do not owe their mechanical character to the life of the whole. What death destroys is not the weight or the mass of the cells, but the capacity for that combined operation by which, could it begin again, the life of the individual would at once be restored. The elements of the living being, that is, are in part of mechanical character, and so far as they are mechanical they persist unimpaired by the fate of the individual whom they have constituted. But so far as they are truly organic their character depends on the life of the whole.

3. Whether the living can be evolved from the inanimate is an unsettled question, but, if it can be, the conditions which render it possible emerge from these considerations. We must assume that some or all of the real elements which constitute mechanical systems have capacities of action other than those exhibited in their mechanical behaviour. Then there are two possibilities. It may be that as long as any such element remains bound in a mechanical system this element of its energy is tied, and cancelled by opposing forces. It is, therefore, potential alone. But if by some regrouping of elements the opposing forces are separated, each is set free, and new forms of behaviour arise. It may be again, that two or more centres of energy which while apart operate upon all other things indifferently, i.e. mechanically, are so related that on coming within the sphere of mutual influence they form a whole in which the action of each is so modified by the other as to contribute 
to the maintenance of the union. In the one case the first formation of an organic unity would be due to the break up of a mechanical system, in the other to a synthesis of elements, just as in the actual evolution of the organic world, as well as in the evolution of mind and society, the foundation of a new type is laid either by differentiation from a pre-existing whole or by a synthesis of pre-existing elements.

The organism once formed moves, like any structure, on a path prescribed by its internal arrangement. It is regardless of everything else, except so far as affects its own fate. Indeed, any symptom of a correlation of its behaviour with that of others may be taken as an indication of the beginning of a new synthesis, whereby the organism will enter as a constituent into a higher organism. But as compared with the mechanical structure, two main points have to be noted in the behaviour of the organism. The parts being adaptable to the needs of the whole, the organism has flexibility, and its line of action takes the course of a regular development, reaching in the physical organism a time of maturity, followed by a break up in the case of unicellular organisms, or decay and death in the case of higher organisms. Secondly, in maintaining and developing itself, the organism lays hold on the outer world, converting the energy of the environment, in the form of food, into energy subservient to its own needs, and in greater or less degree rearranging the environment generally in such wise as to further its own ends. This is not organicity, but organisation-organisation being the arrangement of elements that remain mechanical with the view of producing certain ends. Such organisation is the servant of the organic principle, which by means of it secures its own development.

We find then in organic development three moments or distinguishable sets of conditions.

i. In the formation of any new organic type there is a synthesis of elements previously separate, and in their separateness either held ineffective by counteracting forces or, if acting, acting mechanically for simple lack of that with which they can harmonise.

ii. In the individual or the type so formed these ele- 
ments, acting in a determinate relation, have a certain scope of operation, the filling up of which constitutes the development of the individual.

iii. Both as a condition and result of this development elements of energy originally foreign to the organism are absorbed and arranged so as to subserve the organic movement.

It is only in the second 'moment' that we find anything like a 'pre-formation' in the germ of the mature individual, and even here there is not necessarily any real identity of character, though there is true continuity of individual being. What must exist at the beginning is not the developed structure in miniature, but rather something that will seize on all that comes within its grip and throw it into place in such fashion that bit by bit the structure will grow. As in a country dance a person standing at a certain point and giving his hand to dancers in succession will swing them round in a definite direction, and so produce in the end a new formation, so we may conceive the organic system dealing with all that comes to it, and after selecting what it can absorb and extruding what it cannot, throwing each item that it retains into the position in which it will form part of the matured order. For this purpose the germ need not be in the least like the matured order. It must only have a mode of operation, which is determined by the needs of that order. In this respect it resembles a purposive idea, and, in fact, as we have seen, there is an element of purpose involved in organic action as such. But it is not until the higher levels of development are reached that it becomes a fully articulate purpose guided by an idea. At lower levels an organic element reacts to each situation in such wise as best to maintain the union of the living whole, and in the course of growth, as each element is added, the addition is so made as to preserve, while it unavoidably modifies, that union. The action of each element at each moment is directed to the maintenance of the equilibrium of the next moment, and the arrangement of the organism is such that this process does not defeat itself, but leads on and on to a point of maturity. Beyond this point, on the other hand, the organism cannot advance. 
There is no further development except through a new synthesis.

4. Of the causes which bring about such a synthesis we know little, except in the higher stages of development, where it is the deliberate work of Mind. Yet a new synthesis, with whatever modification it may involve, is often for the organism the only alternative to destruction. Each organism proceeds in the path of its development, just as each member of a mechanical structure moves in the orbit marked out by the conditions of the structure, without regard to its effect on other organisms or other structures. Hence discord, disorder and, at a higher remove, the pain and suffering which are disorder rendered in consciousness. There is, however, from the first this difference between the organism and the mechanical structure, that the organism can adapt itself within limits that gradually expand to new circumstances, guard against dangers, and even in some degree remodel itself so as to avoid or to soften the shock which would otherwise destroy it. It is not regardless of the foreign body so far as the effects on itself are concerned. It is, however, so long as it is fully separate, regardless of its own effect upon the others. Hence the clash of organic forces and the struggle for existence. The only escape from this struggle lies in the disengagement of new forces, which, modifying each organism, lay the basis of a new synthesis. This is the regular work of the higher forms of mental activity, which move consciously towards a harmony that is to leave nothing outside its limits. At a lower stage the process is fitful and uncertain, depending on the general condition that a new synthesis can only occur when elements fitted to unite with one another are brought into a favourable conjunction. But, as has been remarked in a different context, there is this general condition making for harmony, and therefore for development, that so far as organisms, or indeed any structures, come into conflict, they tend to arrest, cancel and destroy one another, while conversely, so far as harmony extends, they tend to maintain and further one another's development. 
Development in general then is an extension of harmony in activity. ${ }^{1}$ In the undeveloped state forces are locked in conflict, they cancel one another. There is mutual arrest and stagnation. The first act of development may be considered as liberation of energy, through a rearrangement which enables colliding forces each to produce a definite series of changes, but if there is to be true development and not mere disruption the forces set free still remain related. They act on one another only not so as to cancel one another, but so as to engender the correlated movements of an orderly structure. This is the second act. But some structures are of organic type because there exist elements capable of so working together as to maintain and develop one another. As long as they remain separate these forces are either held cancelled by others or operate on their environment in mechanical fashion. As soon as in the continual movement and redistribution of things each meets with its mate they form a whole of the organic type with the power of maintaining itself by plastic adaptations. The adaptation enables it to absorb or subordinate external sources of energy, and so to grow. This is the third act. But each organism still acts indifferently, i.e. mechanically, on the remainder until either (I) it meets its 'fellow,' when once more a higher unity is formed, or (2) in the process of its growth and internal modification it reaches a stage at which it is fitted to act in harmony with others to which it was previously indifferent. In either case the lower organisms are the basis of the higher, and development involves a repeated synthesis and harmony, which constitute the remaining acts of the drama.

Lastly, as has been shown above, harmony is not only a product but a condition of development. Any structures which are incompatible with one another must cancel out and destroy one another as they come into contact, and all

${ }^{1}$ As opposed to the development of a particular thing, which means simply the more complete realisation of that thing. In the case of Mind as that which is based on a harmony and is the basis of a fuller harmony, the two meanings express different aspects of the same process, the fuller realisation of the potentialities of Mind being effect and cause of a deeper and more extended harmony. 
the lower organisms which are mutually indifferent structures are thus destroyed in immense numbers. In the harmonious whole, on the other hand, the elements instead of cancelling maintain one another, and if the whole consists of organisms each capable of development the harmony involves mutual furtherance of such development. At the same time it must be observed that related organisms may have each more than one possible line of development, and that among them those which conflict will destroy one another, while those that harmonise will survive. Thus (I) a harmonious whole has an advantage over others, and (2) a partial harmony tends to become a complete harmony. In both ways harmony is a self-multiplying process, and though a higher unity is always liable to destruction by lower ones which it has not incorporated, yet over long periods the permanent make-weight has its effect, and there is a progress of development, which is complete only when the whole field of reality is subdued to the needs of a single organic whole.

The whole process must, in accordance with the conclusions of the last chapter, be referred to a teleological impulse working through mechanical conditions. Everything that exists must be conceived as determined by, as owing its existence and character to, the contribution that it has to make directly or indirectly to the ultimate harmony of the whole. But in accordance with the same conclusions each constituent part of reality, in so far as it is separate from others, maintains itself, and if it is a living being tends to grow and expand indifferently to other things. In this effort it impinges on and conflicts with other elements similarly impelled, and hence there is disharmony and evil. As conflicting tendencies cancel out and those which harmonise are extended, harmony grows. The completion of the process involving modifications proceeding through the entire complexity of things occupies vast spaces of time, and throughout this time disorder persists, though order advances. Thus every element of reality is determined by the function which it performs at some stage in the formation of the organic whole, but until that whole is completely established may 
remain in greater or less disharmony with other elements. For each element has its function in some specific structure alone, each structure in some specific higher structure, and except in this appropriate synthesis there is always disorder. Development consists in synthesis upon synthesis, and until the process is complete discord remains.

5. In the higher organisms the work of establishing new correlations, and therefore in particular the work of adapting the organism to a higher synthesis, is the function of Mind, and in particular of that union of Mind-functions which constitutes consciousness. The growth of harmony becomes, if it is not from the first, identical with the growth of Mind. Of the beginnings of Mind we can get no direct empirical evidence, because a mind-function is not something which can be directly seen or touched. But applying to Mind the general considerations as to Development, which have been explained, we regard it essentially as a mode of activity dependent for its specific character on the co-operation of elements. These elements, as long as they exist apart, would not constitute the peculiar form of unity which is Mind, but would be related to it as the chemical molecules which constitute a cell are related to the living cell. If these molecules come together to form a cell, they undergo some development either by the unlocking, or by a mutual modification in the action, of preexisting energies, and if that cell is conscious, the preexisting energies must be conceived as containing or exerting activities which unite to form the activities of consciousness, just as they exert pressures and tensions which in combination yield the phenomena of contractility. That is to say, mind in the organism is not to be conceived as either external or as growing out of something like matter, taken as wholly discrepant from it. It is to be conceived as a synthesis of elements, which do not function except in combination.

6. Thus the growth of harmony involves the evolution of individual minds, which constantly enter into deeper and wider relations with one another. But beyond this our 
account appears to imply a permanent activity of a Mind that is not limited to a single physical organism. For at least so far as our experience and our powers of conception extend, the existence of a Purpose implies a Mind commensurate with that purpose. Mind is the permanentwe may venture to say the substantive-basis of purposive conception or activity. Where we trace germs or filaments of purpose we infer the rudiments of mind. Where a purpose of given scope is plain there is to be inferred a mind of not less scope. If, as we now conclude, a purpose runs through the world-whole, there is a Mind of which the world-purpose is the object. Such a Mind must be a permanent and central factor in the process of Reality, but how in detail its relation to reality in general, and the individual mind in particular, is to be conceived is a question about which it is best frankly to confess ignorance.

When we seek to embody the conception of a conditioned teleology in some concrete expression, the images that we have to use are drawn from a limited experience, and the danger is that instead of expanding them to the measure of the broad principles which we seek to elucidate, we may treat them rather as cast-iron moulds into which the wider thought has to be compressed. Thus we may picture the process of the world as the work of a Mind moving towards a fuller self-realisation by subduing the conditions which limit it. But if we speak of 'a Mind' and ' a Self' to be realised, the terms inevitably suggest a personality like our own, a mind shut up within a body, a self exclusive of other selves and yet related to them. Pressing this view of unity, we tend towards a Dualism which would be inconsistent with the principles which it intends to formulate. If again we seek to subdue Dualism to the supremacy of the spirit, we are in danger of falling into a colourless Monism devoid of all the life and motion that depend on the interaction of personalities, with their loves and hates, emotions, which must, for their very being, know the gulfs made by seclusion and separateness, that they may overleap them. If again, in reaction from Monism, we assert the independence of the organic constituents of the whole, we may drift towards the notion 
of a world of self-subsistent separate selves, in which unity disappears into a merely abstract relation.

In all these cases the root of the trouble is that we are seeking to force a thought derived from abstract reasoning into the shape of concrete images which were not made to fit it. The general considerations derivable from our analysis of the ideal of thought point to a certain conception of the world system, the conception which we call organic, which involves a conditioned purpose, and a development wherein that purpose advances towards realisation. If we look to the synthesis of experience we find a corresponding conception slowly building itself up as we compare the successive stages of evolution. There, too, we find evidence of Mind emerging under conditions of which by degrees it becomes master, and in mastering them comes to the fuller realisation of its own capacity. But in tracing this process the wealth of the experience which we bring together forces us to realise the imperfection of the terms in which we seek to render it. The kind of unity, for example, attributable to 'Mind' in this relation could by no possibility, as long as we keep the empirical sources clearly in view, be confused with the mind of a human individual. We are forced to conceive wider and more elastic possibilities of unity, to recognise that there is a connectedness which makes a family, a class, a church, a nation, mankind, unities for certain purposes or in certain relations, that, moreover, such unities may grow and come to dominate the entire life of the component members, that they depend in part on conditions which subsist though none may know them, and in part again on the very fact that they are recognised. Such considerations are but a hint of the complexities of relation implied in such a term as the spirit of a class, or the soul of a people. Such expressions we realise are justified if we have none better, for we have to deal with the unity and refer to it and discuss it. But they are not justified if they are so made a basis for inference that characteristics are attributed to the soul of a people because they are true of the soul of one man. So again we may speak of the Soul of the world, of a spiritual principle working towards self-realisation, as long as we 
have no better method of grouping the legitimate results of reasoning and experience into a single term, but we must be exceeding chary of clothing the conception further with attributes drawn from the soul as consciousness reveals it within us. We shall do better to devise fresh terms clear of old associations and better fitted to express a more complex experience, and we shall perhaps do best by seeking the further expansion and interrogation of our empirical synthesis, wherein in proportion as it expands new categories, more elastic, more general, less inadequate, evolve themselves as the result of the wider and more articulate correlation of data.

One negative limitation indeed is clear. The Mind that we are led to contemplate must neither be confused with the whole of things nor with an Omnipotent Creator of things. It is not the whole, for mechanism-the antithesis of purpose-runs through the structure of the whole, and in dependence on mechanism, discord and evil. It is not, therefore, to be confounded with the Absolute or Unconditioned of Metaphysics. If these terms have meaning, they possess it only as applied to the whole, and in the whole Mind is only a factor. It is conditioned as its Purpose is conditioned. For the same reason the Mind to which our argument points is not the Omnipotent Providence of a more elementary religious theory working at its will in a void or on a material of perfect plasticity. The reality of evil must be recognised as something very different from a mere privation of good. It is the positive result of the clash of processes, and of purposive processes, too, that are not organised. Its extent is the measure of the incompleteness of the order actually achieved by Mind in the world.

7. It may be reasonably asked whether even this statement is exhaustive. Can the catastrophes of earthquakes and floods, the more loathsome diseases, or the extremes of moral turpitude be reconciled with a plan in which everything at bottom is to serve some purpose in the harmony of the whole? On this point it may be remarked (I) that it is not here suggested that every event is good, but only 
that every event proceeds from some combination of forces, each of which is somewhere or some time necessary for the fulfilment of the world-purpose. The evil that these forces may work is the price that is paid for them, and that this price has to be paid is the proof of the limitation of purpose, not of its non-existence. This being understood, the very fact of the callousness of nature is the best testimony to the general account here offered of evil, that it is the outcome of the blind operation of mechanical forces. (2) In relation to moral evil it is sufficiently clear, with regard to the mass of normal wrongdoing, that it is the result of the pursuit of partial ends without regard to the effect on others. Selfishness of the individual, or selfishness of the family, class or society is at its root, and the characteristic of all such selfishness is that while its end may as an end be blameless and even laudable, it is its limitation that makes it bad by impingement on the equally just claims of other individuals or groups. Here again evil is simply the result of the inorganic relation of human beings or human societies. There remain the cases of monstrosity, of cruelty, treachery and aggravated lust. These, which seem to a simple and unreflective experience to be clear evidences of a Satanic Mind, are more and more clearly reducible by psychological investigation to pathological growths, by which the normal mental structure is obsessed or distorted. Impulses that are natural and necessary acquire a morbid predominance, or take a perverse twist, and this again is due either to an unhappy combination of hereditary tendencies in the constitution of the individual, or to the destructive operation of experiences to which the character has been unable to adapt itself. It is only in melodrama that men are allround villains glorying in their villainy. The tragedy of actual life is that under the stress of overwhelming temptation or mastering impulse men do vile things who in their normal selves are sufficiently good members of society.

8. Thus from two opposite starting-points we have arrived at the conception of a conditioned purpose as constituting the core of the world-process. The analysis of 
thought points to the conception of the Reason as an impulse to secure harmony of conceptions, an impulse which can only be finally validated by development. The analysis of the ethical consciousness points to a goal of effort in which the harmony of all conscious life is to be attained. When, further, the postulates of rational thought are carefully examined, they suggest that this harmony is not a mere ideal, but a just description of the goal to which the movement of the world tends, and this leads us to infer a power of the nature of Mind operating under conditions towards the effectuation of a world-purpose. But it is precisely to this point that we had been led independently by the synthesis of experience. The theory of evolution began with the biological order. It showed first how all forms of vegetable and animal life might be conceived as issuing from a single origin. This conception is now undergoing extension at both ends. Physical science is extending the principle of development to the inanimate. It is coming to regard not merely the specific forms of matter as variants of a common original, but matter itself as a structure evolved from a more primitive source. On the other side psychology and sociology are busy exhibiting the higher forms of the superorganic world and tracing the phases of development experienced by the individual and the social mind. There are doubtless great gaps remaining in the scheme. In particular, the transition from the inanimate to the animate is not made out, and can only be tentatively imputed to a synthesis on the analogy of better known cases of the appearance of a new kind. But there is no reason to doubt the substantial validity of continuous development as connecting the lowest with the highest orders of being. The principal object of our enquiry has been to determine in what development consists, and here, as the result of a purely empirical synthesis, we were led to the conclusion that it consists in the growth of Mind. To measure this growth we distinguished a succession of phases, and we found that in each phase the transition was effected essentially by the gathering into the scope of purposeful mental activity of conditions that were already in operation from without at the lower phase. The highest 
known phase we decided to be one in which the mind of humanity, grasping the conditions of its own development and the true goal of its action, opened to itself the prospect of dominating the actual future of the race and securing the harmony which is its ideal. That this prospect was not a bare idea, but rested on real conditions rendering its realisation possible, we showed by the consideration that development in general rests upon harmony, and arrest upon conflict and incompleteness of organisation, and that in the rise of mind-power to the point in question the general condition necessary to the completion of harmony and avoidance of conflict was fully given. We could not, however, on this ground decide on the position of the social mind of humanity - a product of one planet of our solar system - in the world, and for this reason, if for no other, we had to enquire what general considerations applying to Reality as a whole could be brought to bear upon the problem. Starting from these general considerations, we were led to infer a development precisely parallel to that which our synthesis had yielded-a development of harmony which constitutes the gradual realisation of a conditioned purpose.

In one point indeed the deductive argument does not at first appear to square with the empirical conclusion. It leads us to conceive the operation of Mind as permanent, whereas the facts of development point rather to its gradual evolution. But on closer inspection the discrepancy disappears. For (I) as hinted at an earlier stage of the argument, if we persevere with the organic conception, we must regard the central mind ${ }^{1}$ itself as undergoing development. If it is conditioned as well as condition, it must be limited by the constitutive elements of the Real unity, and in so far as it has not dominated them, must be dominated by them. Its evolution, in fact, proceeds through those processes of organisation and synthesis which have been indicated here, and which correspond, in general tendency, with the stages of development

${ }^{1}$ It follows-in opposition to a more mechanical teleology-that the Purpose operating in evolution is itself not fully defined from the beginning, but susceptible of development. 
revealed by the empirical synthesis. Accordingly, (2) Mind, as we know it empirically, whether in the individual or in the group, is the product, and so far as it is truly mind, is deservedly reckoned a true constitutive part of the permanent mind. Its existence depends on mechanical conditions, on a cerebro-neural structure for one thing and on complex physical and social relations between individuals for another, the shaping of which is precisely the work at which a mechanically-conditioned purpose is for ever busy. Thus Humanity, in the sense which the best Positive writers have given to that word, Humanity as the spirit of harmony and expanding life, shaping the best actions of the best men and women, is the highest incarnation known to us of the divine. If, indeed, we come to the conclusion that God is, and are asked what $\mathrm{He}$ is, we may reply that God is that of which the highest known embodiment is the distinctive spirit of Humanity. And of this account of the relation of the empirical to the central mind there is in the empirical account itself more than a hint. For at each stage we have shown that the conditions of a higher stage are already present. It is not the mere empirical mind itself that works out its own progress. It is the empirical mind operating upon other conditions of progress that are already laid down. The human mind is a germ for whose maturity provision is already made. Furthermore, at the highest known phase of development we say that the mind comes to realise itself, that is, to realise what are the fundamentals of its structure as it has been all along. In this new consciousness it discovers a unity underlying the differences and divergences of life and a plan containing the possibilities of a future self-realisation. It does not invent this unity and this plan. It discovers them. It finds that they are already there, and have been among the conditions operating to determine its growth from the earliest stages. Its own purposeful activity is merely the continued operation of these conditions completed by the unifying link of the consciousness of their significance. Hence, if the mind does not directly through the religious consciousness become aware of its relation to a greater Spirit, it does have to recognise the 
existence of conditions appropriate to the operation of such a Spirit, and to admit in its own history a process in which such conditions are working out their natural results.

Thus, broadly viewed the two lines of thought are in close agreement. Both lead us to conceive the worldprocess as a development of organic harmony through the extension of control by Mind operating under mechanical conditions which it comes by degrees to master. The empirical synthesis is in the main limited to the history of mind upon this earth, and to the stages by which intelligence makes for itself a vehicle in the physical organism. The deductive argument exhibits this process as a part of a vaster and more significant evolution. But the strength of the position is that, so far as the two arguments cover the same ground, they coincide in the main lines of their teaching. The conclusion which they yield by no means answers all the questions that men ask of experience. But, if it is sound, it does settle the fundamental questionswhether the life of man is full of hopeful purpose or void of meaning, whether he can recognise in the constitution of things something that meets his hopes and answers to his aspirations, whether he can make for himself a religion without self-deceit, whether he can finally improve the condition of his race by effort or is doomed always to fall back from every apparently forward step, whether he can trust to his reason or must admit the ultimate futility of thought, whether the spirit of human love is justified of her children or blood and iron must continue to rule the world. To all these questions the conclusion here reached supplies a definite and a positive answer. It is, however, maintained here, not as something which is to satisfy all emotional cravings or end all intellectual doubts, not because it is artistically complete or even because it is proved with demonstrative certainty, but merely on the humble and prosaic ground that, on a complete and impartial review of a vast mass of evidence, it is shown to be probably true. 


\section{INDEX.}

\section{A}

Absolute, The, 367 .

Abstraction, I 4I, 145 .

Action (see also Conation, Consciousness, Correlation, Mind, Reflex Action, Type-reactions, Stimulus, Purpose).

Acclimatisation, 6r.

Adaptation, 50.

Assimilation, 64 et seq., 84 .

Enduring organic effects, 6 I.

Equilibration, 51 .

Instinct, 56, 88.

Mechanical theory of, 5, 6 .

Non-correlated, 45,46 .

Purpose, 50.

Selective modification, 62, 63 .

Sensori-motor, 52 et seq., 88.

Correlation:

Articulate, 70, 80.

Conscious, 72, 79, 80, 8r, 83, 85,87 .

Inarticulate, 88.

By heredity, 46 et seq.

Structural and reflex, 87,88 .

Unconscious, 73, 79, 80, 81 . Adaptability, Organic, 306, 322, 359.

Affection, 216.

Agriculture, 214.

Algebra, $15 \mathrm{I}$.

Amoeba, 56.

Analysis and Synthesis, 39, 80, 105 , I 27, I $30,131,1$ 32, I 33, I 35 , I $36,140,141,143,145,146$, 147, I 48, 241 et seq., 244, 245, 259 .
Ancient Philosophy, I I 4, I 15. Animal Consciousness, 44, 64 . Animism, 95, 98, 99, 102, 103, $183,215$.

Anticipation, 75, 77, 79 .

Arabic schools, $15 \mathrm{I}$.

Arahat, 120.

Archaeology, 205.

Archimedes, 12 5, 126, 212.

Aristarchus, 126.

Aristotle, 1 24, I 50, 151 I 161, 163 .

Arithmetic, 105, I14.

Art, 91, 108, 190, 191.

Development of, $216,217$.

Idealistic, 217.

Progress in, 218, 219.

Articulate Experience, $247,248$.

'As such' relations, 260 et seq.9. $342,343,344,348$.

Assimilation, 64 et seq., 69, 84 .

Assumptions of Science, 332.

Astronomy, 1 24, $125,126$.

Attribute and Substance, 95, 96.

Aurignacian Man, $216,217$.

Authority, 22 1, 222, 226, 227.

Axioms, 159, 259, 265 et seq.

\section{B}

Babylonia, I14, 123, 205, 211.

Bacteriology, I I O.

Barbarian Invasions, 229.

Barometers, 213.

Basis of Moral Order, 200.

Beauty, 140, 218.

Belief, 97, 98, 99, 155 .

Finality in, 276, 277.

Benevolence, 225. 
Berkeley, I 53, I $57,236$.

Berry, C. S., 78.

Biology, I25, I26, 197 .

Biology in politics, 9, 10.

Biological conditions of development, 289, 290.

Blood Feud, I81, I84, I86.

Body, see under Mind.

Brahminism, I I 5 , I I 8 .

Brain, see Mind.

Bronze, 2 I I.

Buddhism, I I 9, I 88 .

Bushmen, 216, 217 .

\section{C}

Calculus, I43, 240.

Categories, 92, 93, 95, 97, 100, $124,236,238$.

Of mechanism, 298, 299.

Of thought, 236, 258.

Cats, 78.

Causation, 101, 102, 1 39, 261, 294.

As continuous process, 298.

Mechanical, 3 I 2, 31 3, 3I6.

Universal, 341, 342, 343 .

Causal tendency, $319,320,327$, 328.

Causes, Plurality of, 262, 346, 347 .

Ceremonial, 96, 98, 99, 104 .

Change, $344,345,349$.

Character, $36,80$.

Chemistry, 2I 2, 2 I 3, 2 I 4 .

Chinese Ethics, I 15.

Choice, 175.

Christianity (see also Religion, Ethics), I I 5, I16, I I 7, I88, I 93, 205 .

Citizenship, 222, 227.

City state, 193 .

Civilisation :

Roman, 227, 229.

Greek, 227, 229.

Modern, 228, 230.

Class, 22 I.

Classes, $83,186$.

Classicism, 217.

Classificatory method, 124 .

Clocks, 2 I 2.

Coal, 2 I 3.
Cole, L. W., 78.

Collective Responsibility, 226.

Colour-line, The, 222.

Common Good, I69, 170.

Common-sense, 92 et seq., I I I, I I 2 , I $7,118,122,217$.

Common-sensemorality, $185,194$.

Community, 178.

Comparative methods, 204, 205.

Comparative Psychology, 247, 369 .

Compass, The, 212.

Conation, 52, 56, 58, 74, 75, 76, 77.

Concepts, $83,84,94,103,127$ et seq.

Function of, 135 .

The logical, 127, 128, 129.

Conceptual Reconstruction, I I 3, Pt. I. ch. viii., I 22 et seq., 217. Conditions of Development, Pt. II. ch. i., 233 et seq., 28 I et seq.

Confucianism, I 88.

Conjectures, $124,125$.

Conscience, 170 .

Consciousness (see also Correlation, Development, Experience, Heredity, Mind, Purpose), 7, I8, 19, 20, 21, 22, 32, 40, $42,54,64,87,88,89,91$, I01, 140, 235, 287 .

Background of, 38 .

Centre of, 22.

Conditions of, 287 .

Consciousness of Animals, 44, 64,79 .

Consciousness as organ of correlation, $32,43, \mathrm{Pt}$. I. ch. iv. and v., 4 I et seq.

Consciousness as epiphenomenon, 8.

Consciousness and the unconscious, $21,247$.

Degrees of, 22, 23.

Developments of, 288 .

Margin of, 22.

Object of, 18, 19, 20, 81.

Reorientation of, $24,25$.

Self-consciousness, $83,85,167$, I 68. 
Consilience, 160, 270, 272, 275.

Construction, 132.

Logic of, 134 .

Principle of, 132, 133.

Continuity, 91, I39, I41, I42, 160, 162, 240, 299, 300, 342, 345 .

In history, 205, 207, 227, 229.

Contract, 225.

Contradictions in Thought, 237 , $238,24 \mathrm{r}, 242$.

Control by Mind, 168.

Copper, 2 I I.

Correlation (see also Consciousness, Experience, Mind, etc.), $3 \mathrm{I}$, $32,33,39,40$, Pt. I. ch. iv. and v., $4 \mathrm{I}$ et seq., I57, I66, $251,253,279,325$.

Articulate, 70, 80, 171 , 173 .

By co-existent conditions, 5 I et seq.

Conscious, $72,79,80,81,82$, $83,85,87,287$.

Correlation based on experience, $6 \mathrm{r}$.

Cross-correlations, 83 .

Governing principles of, 85,87 , $89,92$.

Inarticulate, 62, 172 .

Self-conscious, $83,84,88$.

Unconscious, 65,67 .

Correlation of universals, $80,8 \mathrm{I}$, $85,88$.

Analysis and synthesis, 80 .

Correlation of governing principles, 85 et seq.

Direct and indirect, 80.

Corroboration, $26_{3}, 264$.

Crete, 2 I I.

Custom, I 79, I 80 et seq.

Law and custom, 184 .

\section{D}

Darwin, 255.

Deduction, I 24, 25 I.

Democritus, 125.

Des Cartes, 152.

Desire, 70, 7 I, 80, I 73, I 75 .
Development (see also Consciousness, Mind, Progress, Purpose), 90, 91, I10, II I, 162, 164, x65, 166, I95, 196, 197, 199, 200, 203, 227, 280, 336, 337.

Conditions of, 28 I et seq., Pt. II. ch. i., 233 et seq.

Biological, 289, 290.

Physical, 289, 290.

Artistic, 216, $217,218,219$.

Conscious, 288.

Ethical, 215, $216,219$.

Mental, 215.

Social, Pt. I. ch. xi., 204 et seq.

Development and harmony, Pt. II. ch. v., 352 et seq.

Development and validity, 90, $276,277$.

Dialectics, I 23, I27 et seq., 14I, I 44,154 .

Differentiation and Integration, $216,352,353$.

Discontent, 84 .

Discrete, The, I 41, I43, 144, 240, $24 \mathrm{I}$.

Disharmony, 350 .

Dissipation of Energy, 291.

Distinct centres of Social Evolution, 228.

Dogma, $147,148,149$.

Dogs, $77,78,79$.

Dualism, 108, I I 2, I I 8, 1 53, I 54, I $80,184,207,225,334,336$, $34 \mathrm{I}$.

\section{$\mathrm{E}$}

Effort, $5 \mathrm{I}$.

Ego, $2 \mathrm{I}$.

Egoism, 177 .

Egypt, I I 4, $123,205,2$ I I, 2 I 2.

Eleatics, I 23.

Elements, I, 80, 81, 82, 83 .

Empedocles, 125.

Empirical Order, I I2, I I 8, Pt. I. ch. vi., 90 et seq.

Empiricism, 159.

Energy, 283, 344, 354, 355, 356, 357,360 . 
Energy, Dissipation of, $29 \mathrm{I}$.

Epi-phenomenon, 8, 292, 294,322, 323.

Equality, 207, 208.

Equilibrium, Organic, 52, 58, 62 . Error, 274, 276.

Ethics (see also Christianity, God, Morals, Religion, Philosophy), I 16 , I 17 .

Basis of ethical order, 181,182 , 279.

Ethics and religion, 200, 20r, 202, 215 .

Ethical development, 117 , I 8 , I 80.

Ethical idealism, I 5 .

Ethical philosophy, I90.

Ethical progress, 202.

Ethical theory, 169, I 70.

Greek, 192, 193.

Primitive, 181 .

Religious development, 2 I 5 .

Evolution (see also Mind), 4I.

Biological view of, 1 et seq.

Evolution of mind, 108, 150.

Social evolution, 207.

Evolution and Teleology. Pt. II. ch. v., 33 I et seq.

Euclid, 84, I 24, I 25, 165.

Euripides, $218,228$.

Existence, Continuity of, 83 .

Existential Judgment, I 28, I 29.

Experience (see also Perception), $34,43,62,69,70,72,75,76$, $79,80,81,82,83,85,86$, $88,92,97,103,112,124$, I 26, I $34,135,136,146,156$ et seq., I 75, 204, 235, 237, 245, 246, $247,248,25$ I, 258, 292, $331,332,335,337,339$.

Correlation of, 242,248 .

Heredity and experience, I70, I 7 I, 175 .

Pure, I 16, 157, 158, 251 .

Experience and reality, Pt. I. ch. i., 233 et seg.

Experiential Reconstruction, Pt. I. ch. ix., 150 et seq., 217.

Experimental Methods, I 23 , I24.
Experiments, 159 .

Explanation, 320 et seq.

\section{F}

Faith, I 7 .

Family, The, 223, 224.

Federalism, 22 I.

Feeling (see also Social Feeling), $57,64,66,74,79,97,146$, I 47, I 70, I 72, I73, I 77, I 80, $249,277,278,279,318$, 325 .

Feud, Blood, 18 r.

Finality in Belief, $276,277$.

Force, 256, 257, 354, 355, 356, 357,358 .

\section{G}

General Functions of Mind and Brain, Pt. I. ch. iii., 29.

Generalisation, 105, I 57, 25 I et seq.

Geometry, 105, I 14, I 24.

Given, The, 75, 157, 158, 161, 236.

Glass, 2 I 1 .

God, I I 8, I85, I87, I88, 37 I.

Gods, $103,104$.

Good, The, 334, 336.

Government, 219.

Gravitation, Law of, 254.

Greece, 205.

Greek Civilisation, 229.

Greek Ethics, 192, 193, 202.

Greek Philosophy, I 23.

Group Morality, 186, 188, 222, 224.

Gunpowder, 2 I 3.

$\mathrm{H}$

Habit, 69, 73, 78, 88 .

Haggerty, M. E., 78.

Hamilton, G. van T., 78.

Happiness, 84, 169, I96, I 97, 198, I99, 230.

Harmony, I I 7, I69, I 70, I 98, I 99, $228,275,276,277,278,279$, 280,284 et seq., 336,348 , $349,350,35 \mathrm{I}$. 
Harmony :

Development and harmony, Pt. II. ch. vi., 352 et seq.

Harmony and organicity, 312, 313.

Reason as harmony, 249.

Hebrew Prophets, II 5, 187.

Hegel, 154.

Heredity (see also Action, Consciousness, Evolution), 34, 35, $36,69,85$.

Heredity and experience, 34,37 , $38,50,55,56,59,170,17$ I. Hereditary factor, $170,171,175$. Hero, I 26, 212.

Hipparchus, 126.

History:

Continuity of, 205, 206, 207.

Limitations of historical method, $14,16$.

History and philosophy, 233 et seq., 282, 369 et seq.

Holmes, Professor, 67 .

Humanity, I 88, 37 I.

Hume, $153,236$.

Huxley, T. H., 290, 291.

Huyghens, 2 I 2.

Hypotheses, 125, 160, 252 et seq.

\section{I}

Ice Age, 2 I I.

Idealism (see also below), I53, I9I, 193, 194, 197, 217.

Religious, 186, 200.

Subjective, 158.

Ideas (see also Consciousness, Correlation, Feeling, Mind, Idealism), 66, 71, 74, 75, 77, 79, $80,82,91,153,219,319$.

General, 77, 80 .

Identity, I 37,138 .

Images, 77.

Imaginary Expressions, 1 28, 129.

Imagination, 81.

Implications of Reasoning, 264 et seg.

Impulse, I 73, I74, I76, I80, 318 , 319.

Feeling, 97, 99.
Impulse :

Morbid, 368.

Rational, 332, 337, 338, 340.

Indian Philosophy, I 5 I.

Individual, The, $177,225,226$.

Individual and community, r94, 195, 196, 197, 198, 199, 225.

Individual relationships, I75, I 76 .

Induction, 159, 160, 317 .

Scientific, 362 .

Industrial Revolution, 2 I 3,2 I 4.

Industries, Stages and development of, 213,214 .

Infanticide, 194.

Inference, $7 \mathrm{I}, 264$ et seq.

Infinite, The, $163,164,333,349$.

Infinity, 141, 142, 238, 239, 240.

Infusoria, 48, 62.

Inherent Reason, 333 .

Instinct, 37, 171, 172, 322, 323.

Instinct and intelligence, 57,58 .

Integration, 353 .

Intelligence, 113 .

International Law, 222.

Intuition, 38 .

Inventions :

Modern, 291.

Textile, 213.

Iron, Use of, 2 I I, 213.

Irrational, The, 145, 153, 242, 266, $271,275$.

Irrigation, 211 .

Islam, II 5 .

Jennings, Prof. H. S., $48,5^{6}$, 78.

Judgment, 71, 75, 80 .

Moral, I81, I82.

Justice, 177, I 78, 180, 181, 182, I84, I85, 188, 189, 202, 221.

\section{$\mathrm{K}$}

Kant, I52, I 53 , I54, I 57, I $_{58}^{8}$, I $59,164,236$.

Post-Kantian thought, 157 .

Karma, 120.

Kelvin, Lord, 290, 291. 
Kinsfolk, r81, r82, I86.

Kinship, $221,222$.

Knowledge, $1_{52}$, I 54 , I $55,1_{56}$, I64, 206, 209, 274, 275, 277 .

Partial, 242.

Knowledge and reality, 339.

Theory of Knowledge, $237,238$.

L

Language, 70, 79, 82, 90, 91, 1 30 , 176.

Law :

Law and custom, $183, \mathrm{r} 84$.

International, 222.

Moral, I75, 184, I85.

Roman, 224.

Laws of Nature, 193, 195, 196, 202, 253.

Legislation, 219.

Leibniz, 159.

Liberty, 196, 197, 198.

Life, $140,243,302,303,304,305$, $307,309,310,320,358$.

Mechanical view of, 188, 192,

I $93,302,303,304,305,323$, $324,329$.

Limits, I 42, I 43, 239, 240, 241.

Literature, 190.

Lloyd-Morgan, Principal C. M., 64.

Loeb, Professor, 48.

Logic, 98, 124.

\section{$\mathrm{M}$}

M`Dougall, Dr. W., 27.

Magdalenian Man, $210,217$.

Magic, 96, 98, 99, 102, 103, 106, $182,209$.

Malthus, 290.

Marriage, 223, 224.

Mass, 256, 257.

Materialism, I35.

Mathematics, I 24, I 25, I 5 I, I 59, I60, 239, 240, 241 .

Mechanics, mechanism, and mechanical theory, I 25, I34.

Mechanism, 223, 243, 343, 350, $355,356,362,363,367$. Conditions of, 324 .
Mechanics :

Mechanism, Indifference of, 297 , 298, 299, 301, 305, 306, 309.

Mechanism and Teleology, Pt. II. ch iv., 295 et seq.

Mechanical causation, 312, 3I3, 3 I6.

Mechanical continuity, 326 .

Mechanical order, 162.

Mechanical system, 295 et seq.

Mechanical theory, 324,326 , 327.

Of action, 5,6 .

Of reality, $29 \mathrm{I}$.

Mechanical view of life, 26, 27 , $303,304,305,322,3^{2} 3,3^{24}$. Memory, 75, 80.

Mental development in the Individual, $4 \mathrm{I}$.

Metaphysics, I 26, 209.

Metazoa, 63.

Microscope, 2 I 3.

Mill, J. S., 254.

Mills, 2 I 2.

Mind (see also Action, Consciousness, Correlation, Evolution, etc.), $364,365,366$.

Abstract type of, 39 .

Analysis and synthesis, 80 et seq. Biological view of, $\mathbf{x}$ et seq.

Mind and body, 25, 26, 329.

Causal process, $\mathbf{r} 6$.

Mind and consciousness, 20 et seq., 87 .

Control by, 87, 88, 203.

Development of language, 82 .

Mind and its environment, ro8, I 09, I I O, I I I, I I 2.

Mind as epi-phenomenon, 292, 294, 322, 323.

Evolution of, 9, I I, I 2, 3 I, 8 r, $86,87,88,108,1_{50}$, I $_{5}$, 365 et seq.

General, I 66, I 68.

Mind as a factor in evolution, 3I, 281, 282, 284, $288,289,292$.

Function of, 12, 27, 29. 
Mind :

Harmony, 17.

Mind and matter, 322, 323 .

Mechanical view of, $26,27$.

Mind and purpose, 315, 316, $318,335,350,365,369$.

Qualitative changes in, II.

Mind in reality, 16, 233, 234, 235, 292.

Mind as self-conscious, 203 , 288.

Self-consciousness and self-criticism, 84,85 .

Self-control of, 168.

Mind-structure, Pt. I. ch. ii., I 8 , $34,35,36,153,154,156$.

In part a social process, 12,83 , $84,369$.

Social psychology, I 2, I 3 .

World of, 244.

Miracles, 104.

Modern Civilisation, 228, 230.

Modern Inventions, 291.

Modern Philosophy, I I 4, I I 5, 152.

Monism, I I8, I I 9 , I 20, 365 .

Monkeys, 78 .

Monotheism, I I 4, II 5, I I 8 .

Morals (see also Christianity, Ethics, Religion), 174.

Basis of moral order, 201, 202.

Common-sense morality, 185 , 194, 202.

Group-morality, 186, r88, 222 , 224.

Moral judgment, $\mathbf{I} 8 \mathrm{I}$.

Moral law, I76, 184 .

Mother-sense, 38, 39, I47, I48.

Motion, I4I, I42.

Music, 218.

Mutations, 3.

Mysticism, I I 5, I 2 I, I 35, I48, 193, 201.

\section{$\mathrm{N}$}

Names, 82.

Natural Selection, 255, 285, 286.

Nature, 104.

Law of, 193, 195, 196.

Neolithic Age, $210,216$.
Nervous System (see also Action, Consciousness, Mind, Reflex Action, Stimulus), 4, 27, 29, 3 I.

Functions of, 27, 29, 31 .

Structure of, 29, 30 .

Net movement in social evolution, 209 et seq.

Newton, 1 59, 254, 255.

Novel, The, 218.

\section{$\mathrm{O}$}

Object of Consciousness, 18, 19, 20, 80.

Objectivity, $153,154,236,237$, 274.

Order, $35^{\circ}$.

Orders, The Two, Pt. I. ch, vii., I 08.

Omnipotence, 367 .

Organic, The :

Adaptability, 306, 322.

Character, 144, I 45, 244.

Growth, 306, 307 .

Harmony and organicity, 312, $3^{1} 3$.

Mechanical, 144, 145, 146, 309, 3 IO.

Plasticity, 6r.

Purpose, 302, 304, 312, 313 , $315,317,3$ I $8,319,320,338$. Unity, 302, 305, 306, 307, 308, $309,310,311,312,318,336$, $33^{8}, 35^{6}, 357,35^{8}, 359,360$, 362 .

Ostwald, Professor, 254, 355.

\section{$\mathrm{P}$}

Pain and Pleasure, 172, 177, 197, I 98.

Palaeolithic Age, 209, 210,214 , 216.

Parallelism, 8, 228, 329.

Paramecium, 62.

Parental Care, 171, I 76.

Partial Knowledge, 24I, 242.

Particulars, 80.

Past and the Future, The, Pt. II. ch. iii., 28 I et seq. 
Patriarchate, The, $223,224$.

Peace, 84 .

Perception (see also Experience), 34 , $35,54,59,65,69,70,73$, $74,75,76,77,81,82,84$, $87,91,92,109$, I10, 136, $244,258$.

Perfection, 334 .

Personality, 38, 169, 170, 199, 200, 201 .

Philosophy, I08, I14, I1 5, I25, $148,149,150,165$.

Ancient, I I 3, I I4, I I 5, I 23, 124,150 et seq.

Ethical, 190, I9I.

Indian, $15 \mathrm{I}$.

Modern, I I 3, I I4, I I 5, I 50 et seq.

Philosophy and history, $\mathrm{I}$ 7, 233 et seq., 282, 369 et seq.

Philosophical theory :

Necessary for theory of development, 15 .

Physical conditions of Development, $289,290$.

Physics, I 59 .

Plasticity, Organic, 6r.

Plato, I 24, I 51 .

Pleasure and Pain, 172, 177, 197, I 98.

Plurality of Causes, 346, 347 .

Points, I4I, I42, 240.

Polytheism, I 4 .

Positivism, 197, 371.

Practical Reason, 198, 277, 278.

Praise and Blame, I74, I75.

Pre-Socratics, 165.

Predication, 138, 140, 142, I43, I 44 .

Primitive Ethics, 181 .

Principles, Correlation of governing, see under Correlation.

Printing, 213.

Probability, I60, 263.

Progress, 230.

Definition of, 287.

Possibility of, 284 et seq.

As synthesis, 227.

Proof, 268, 269.
Property, 224, 225.

Protozoa, 62, 63.

Psychical Factor, 28.

Psycho-physical Process, 27, 28, 32 , $33,39,41,64,88,170,326$, 327,329 .

Psychology :

Animal, 77, 78, 79.

Comparative, 72, 247, 369 .

Ptolemy, I26.

Punishment and Reward, 174 .

Purpose, 5, 57, 70, 71, 86, 173 , $174,175,244,298,300,3$ r 2 , $313,314,317,318,319,322$. As cause, 324, 325, 326, 335, 336.

Conditioned, 365 et seq.

Mind and purpose, 335, 336, 350.

Nature of, 314, 315, 319, 320.

Organic, 302, 304, 312, 3 i 3 , $315,317,318,319,320$.

Pythagoreans, Í́3.

Qualities, 96.

\section{Q}

$\mathrm{R}$

Raccoons, 78.

Rate, 142.

Rationality, 265 et seq.

Rational impulse, 246, 274, 277 , 279, 332, 337, 339, 340.

Rational interconnections, 263 , 264.

Rationalism, 193, 194, 242, 243 , 264 et seq.

Rational system, 268, 270, 27I, 272.

Reactions, see Type-reactions.

Realism, 189, 217 .

Reality, 84, 86, I I 2, I I 3, I 23 , I 24, I 28, I 29, I 48, I 5 I, I 53 , I $54, \mathrm{I} 56, \mathrm{I} 60, \mathrm{I} 63, \mathrm{I} 64, \mathrm{I} 66$, $167,168,202,233,234,235$, $236,237,258,273,275,277$, 280, 281 1, 292, 33 1, 333, 334, $356,357,363$. 
Reality :

Reality and experience, Pt. II. ch. i., 233 et seq.

Knowledge and reality, 340.

Mechanical theory of, 291.

Reality as a whole, 33I, 333, $337,34 \mathrm{I}, 348,349,350,365$, 370.

Reason (see also Consciousness, Mind, Rationality, Thought, etc.), 84, I I 8, 249.

As harmony, 249.

Inherent, 333.

Implications of reasoning, $26_{4}$ et seq.

Practical, 198, 277, 278.

Recognition, 69.

Reconstruction, 91, 93, 108, I 13 , I I 4, I 22 et seq., I48, 203, 233 et seq.

Conceptual, I I 3 , Pt. I. ch. viii., 122 et seq., I65, 2 I 7 .

Experiential, Pt. I. ch. ix., I 50 et seq., 2 I 7.

The validity of scientific reconstruction, Pt. II. ch. ii., 250 et seq.

Reflex Action, 4, 30, 37, 49, 50, $58,88,323$.

Relations, $79,80,84,85,95,160$, I61.

Apprehension of, 65, 73, 74, 80, 83.

'As such,' 260 et seq., 342 et seq. Individual, 175 .

Variable, 345, 346, 347, 348 .

Relativity, I 52, I 55, I 56, $237,258$. Religion (see also Christianity, Ethics, God, Magic, Morals, Philosophy, Positivism, Spiritualism), I08, I I 2, I I 4, I I 5, I $16,117,165,185,187,215$.

Basis of ethical development, 199, 200, 201, 202, 215.

Dialectic, $\mathbf{r} 20$.

Ethics and religion, II6, I17, 202.

Experience, 1 16, I I 7, 1 20, 200, $201,202$.
Religion :

Idealistic, I87, 200, 202.

Psychology of, I 15 .

Spiritual, 188, $201,202$.

Resemblance, 81, 91, 137 .

Responsibility, Collective, 226.

Revival, 75, 76.

Revolution, Industrial, 2 I 3,2 I 4.

Reward and Punishment, 175 .

Rhizopods, 48, 49.

Rights, 180, 181, $222,224$.

Roman Civilisation, 227, 229.

Roman Law, 224.

Romanism, I 6 .

Romanticism, 217.

Rome, 205, 212.

\section{S}

Sankara, 1 I 8.

Science (see also Causation, Empirical order, Philosophy), I04, 105, I08, I09, I 1 4, I 1 8, I 22, I 23, I 48, I 50, I60, I65, 255. Ancient, $\mathrm{r} 6 \mathrm{r}$.

Applied, 209, 214.

Assumptions of, 332.

Descriptive view of, 255 .

Special sciences, 124.

Units of, 83 .

Selection, $285,286$.

Self, $26,83,84 ; 85$, I $18,180,365$, 368.

Self-assertion, 188.

Self-consciousness, $167,168$.

Self-development, 192.

Self-suppression, 188 .

Sensation, 36, 69, 146.

Sensori-motor action, 66, 69, 70,

$7 \mathrm{r}, 72,73,76,86,328$.

Sensori-stimulus, see Stimulus.

Shepherd, W. T., 78.

Sherrington, Prof. C. S., 27, 3 I,

49.

Sin, 116.

Skill, 54, 55, 67, 88 .

Slavery, 208.

Social :

Co-operation, 226, 227, 228.

Decay, 230. 
Social :

Developments, Pt. I. ch. xi., 204 et seq.

Evolution, 207.

Feeling, 59, 84 .

Mind, 369.

Progress, $227,228$.

Structure, 178, $220,221$.

Union, $220,221$.

Sociology, 125.

Socrates, 123.

Pre-Socratics, 165.

Solitary Wasps, 57.

Sollas, Professor W. J., 2 10, 2 I 7.

Space and Time, 240, $24 \mathrm{I}$.

Special Sciences, 124.

Spiritual, The, 202.

Spiritualism and the spiritual order, I18, I19, 120.

Principle, 228.

Religion, 188, $201,202$.

State, The, 200, 221.

Stentor, 62.

Stimulus, $54,63,64,66,69,72$, $73,76,86,3 \circ 5$.

Structural Activity, 46, 47, 48 .

Structure, 334, 357, 358, 359, $360,362,364,367,371$.

Subjectivity, 275 .

Subjective factor, The, $\mathrm{I}_{52}, \mathrm{I}_{55}$,

$274,275$.

Substance, I 39, 261, 342, 344 .

Substance and attribute, 95, 96.

Supernatural, Io2.

Sympathy (see also Correlation), $173,177,178$.

\section{Synapse, 30 .}

Synthesis, 105, 1 58, 195, 281 , 286, $356,357,358,359,361,364$, $366,367,369,37 \mathrm{r}$.

Analysis and synthesis, I 27, I30, I 3 I, I 32, I 33, I35, I 36, I 40, I 4 I, I 43, I 45, I 46, I 47,148 , 259.

Function of, 259.

Progress as, 227.

System, 91, 93, 1 59, 245, 337, 338, $340,34 \mathrm{I}, 348,35 \mathrm{I}$.
$\mathrm{T}$

Teleology, 162, 363, 365 .

Evolution and teleology, Pt. II. ch. v., 331 et seg.

Mechanism and teleology, 204 et seq., Pt. II. ch. iv., 295 et seq.

Telescope, 2 I 3.

Textile Inventions, 213.

The Two Orders, Pt. I. ch. vii., 108 et seq.

Theory of Knowledge, $237,238$.

Theory of Light, 253.

Thermometer, 213.

Thibaut, George, 118.

Thought, 81, 146, 147, 148, I 55, I6I, $165,25 \mathrm{I}$.

Categories of, $236,238$.

Contradictions in thought, 237 , 238,24 I, 242.

System, 155 .

Time (see also Infinity), 350.

Space and time, 240, 241 .

Totemism, 96.

Tradition, 176 .

Trial and error, Method of, 69.

Truth, 129, 155, 160, 164 .

Tycho Brahe, 126.

Type-reactions, 50, 53, 55, 63, 73 .

\section{U}

Unconscious Inference (see also Consciousness), 69.

Unhappiness, 84.

Unity, Organic, 302, 305, 306, $307,308,309,310,311$, 3 1 2, 318.

Universalism, 193, 202.

Universals, Correlation of, 80, 81 , $83,84,85,86,87$.

Upanishads, I 18.

\section{V}

Validity, 90, 128, I29, I30, 234, $235,332,339$.

Development and validity, 276 . The validity of scientific reconstruction, Pt. II. ch. ii., $25^{\circ}$ et seq. 
Value, 244, 296, 297, 334 . Conditioned, 334 et seq.

Variable Relations, 345, 346, 347, 348.

Variations, 3.

Vedānta system, II 8.

Vengeance, I8I, I82, 183,185 , 22 I.

W

Washburn, Miss, 68.

Weather, Observations of, 80 .
Weismann, 307.

Welby, Lady, 38.

Whitehead, A. N., 164.

Will, The, 59, 84, 180.

The will in development, Pt. I. ch. x., 169 et seq.

Women, Position of, 207, 208, 224.

Wundt, Prof. W. 23.

\section{Z}




This book is due on the last date stamped below, or on the date to which renewed.

Renewed books are subject to immediate recall.

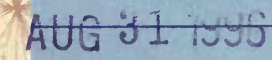

$111+231065$

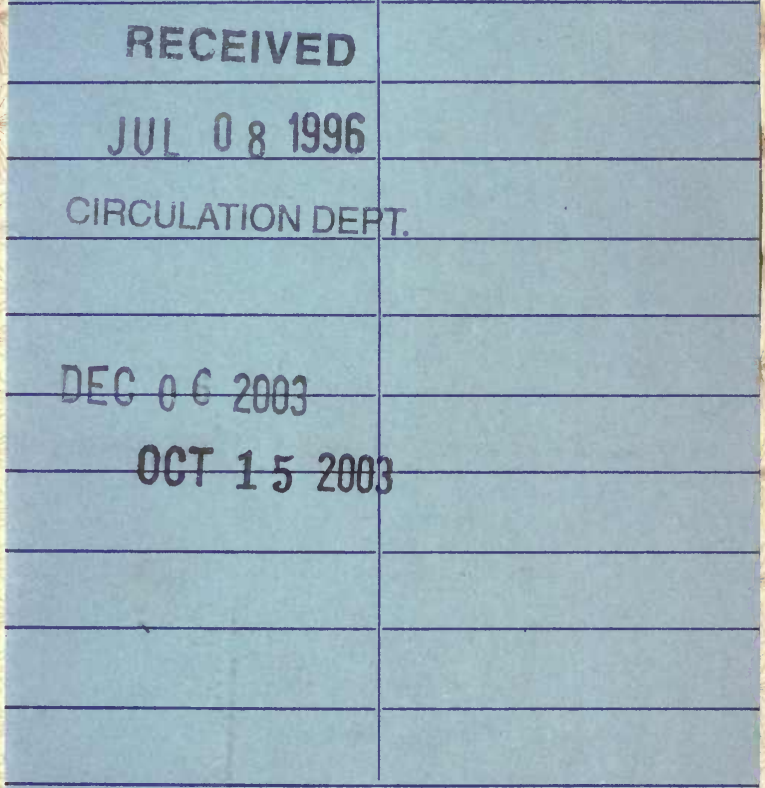

LD 21-50m-12,'61

(C4796s10)476

General Library

University of California Berkeley 
U. C. BERKELEY LIBRARIES

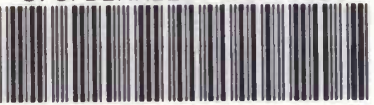

C057122976

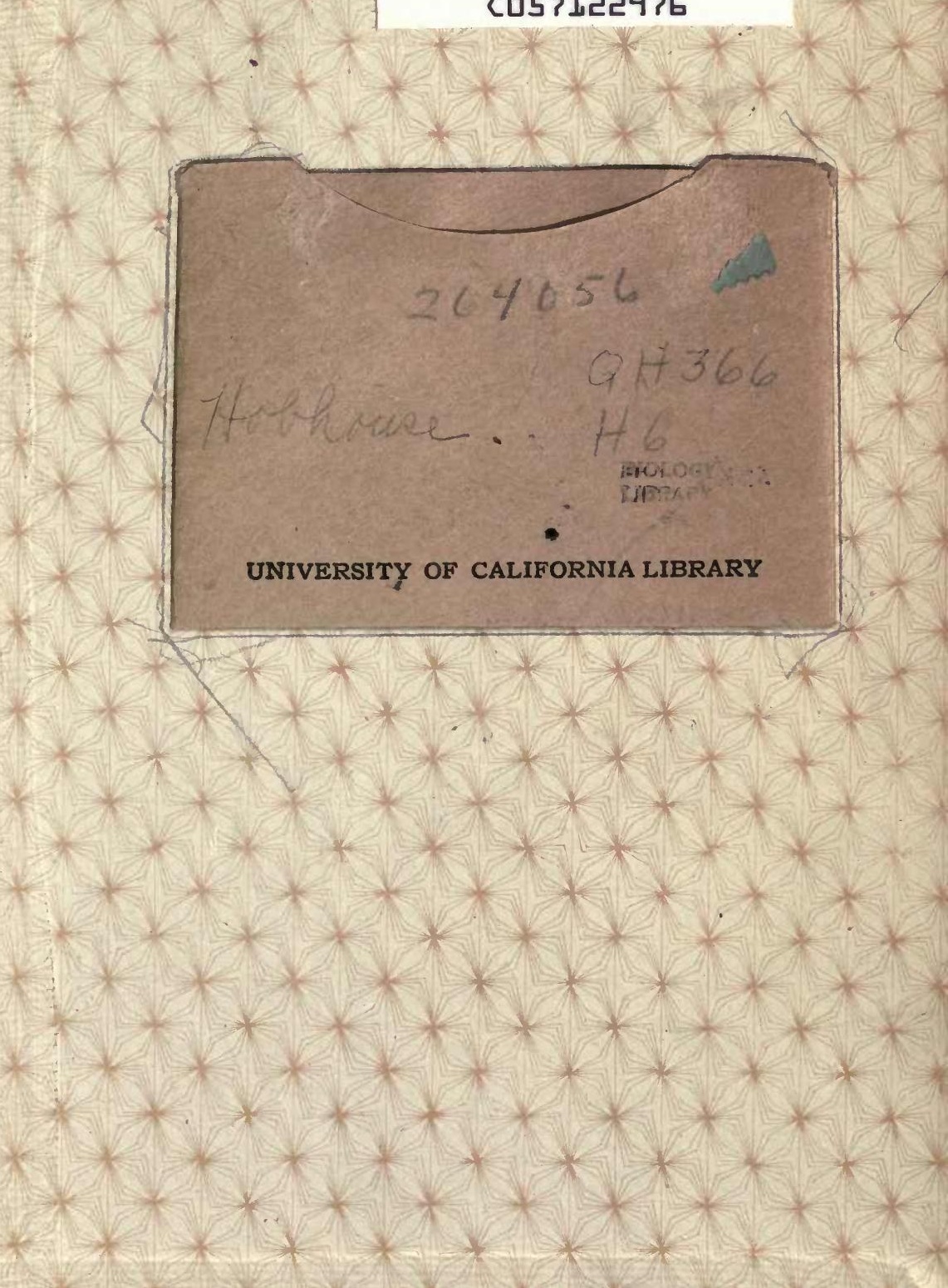


